\title{
Sound Production in Bombycoidea Caterpillars: A Comparative Study of Taxonomic Diversity, Mechanisms and Function
}

By

Veronica Bura

\begin{abstract}
A dissertation
submitted to the Faculty of Graduate Studies and Research in partial fulfillment of the requirements for the degree of Master of Science in Biology

Carleton University

Ottawa, Ontario
\end{abstract}


Library and Archives

Canada

Published Heritage

Branch

395 Wellington Street

Ottawa ON K1A ON4

Canada
Bibliothèque et

Archives Canada

Direction du

Patrimoine de l'édition

395, rue Wellington

Ottawa ON K1A ON4

Canada
Your file Votre référence

ISBN: 978-0-494-71552-9

Our file Notre référence

ISBN: 978-0-494-71552-9
NOTICE:

The author has granted a nonexclusive license allowing Library and Archives Canada to reproduce, publish, archive, preserve, conserve, communicate to the public by telecommunication or on the Internet, loan, distribute and sell theses worldwide, for commercial or noncommercial purposes, in microform, paper, electronic and/or any other formats.

The author retains copyright ownership and moral rights in this thesis. Neither the thesis nor substantial extracts from it may be printed or otherwise reproduced without the author's permission.
AVIS:

L'auteur a accordé une licence non exclusive permettant à la Bibliothèque et Archives Canada de reproduire, publier, archiver, sauvegarder, conserver, transmettre au public par télécommunication ou par l'Internet, prêter, distribuer et vendre des thèses partout dans le monde, à des fins commerciales ou autres, sur support microforme, papier, électronique et/ou autres formats.

L'auteur conserve la propriété du droit d'auteur et des droits moraux qui protège cette thèse. $\mathrm{Ni}$ la thèse ni des extraits substantiels de celle-ci ne doivent être imprimés ou autrement reproduits sans son autorisation.
In compliance with the Canadian Privacy Act some supporting forms may have been removed from this thesis.

While these forms may be included in the document page count, their removal does not represent any loss of content from the thesis.
Conformément à la loi canadienne sur la protection de la vie privée, quelques formulaires secondaires ont été enlevés de cette thèse.

Bien que ces formulaires aient inclus dans la pagination, il n'y aura aucun contenu manquant. 
Anti-predator adaptations have been widely studied in caterpillars, but the majority of studies focus on visually communicated defences. In this thesis I introduce defensive airborne sound production in Bombycoidea caterpillars by documenting the taxonomic diversity and, where possible, function and mechanisms. Fifteen of 42 species from two families and five subfamilies produced sound, providing evidence for both convergent and divergent evolution within the superfamily. Four different mechanisms are reported; mandible clicking, mandible stridulation, whistling through spiracles, and possibly, air expulsion through the buccal cavity. Based on experimental trials with artificial and live predator attacks, I conclude that sounds function to startle and/or warn predators of a chemical defence or general unprofitability. Sound characteristics and preliminary predator trials suggest these defensive sounds are directed primarily at avian predators and possibly bats and rodents. Hypotheses proposing evolutionary origins of sound production are presented based on comparative analysis of signal characteristics and associated defensive behaviours. 


\section{ACKNOWLEDGEMENTS}

I would like to start by thanking past and present members of the Yack lab, the working environment created by everyone's presence is one of the best that I've ever had the pleasure of sharing in. More specifically I'd like to thank Jackie Scott and Sarah Matheson, both of whom started out with different names and helped me through days, and sentences, I thought I would never get through. Thank you for Family Feud, arts and crafts and for taking care of my caterpillars when I was away. To Katie Lucas for checking all my emails and introducing me to sites I still use when procrastinating. Thanks to AJ Fleming (Don Alan!), who helped me with everything from collecting data to acting as a personal translator in Costa Rica, and for always asking the wrong questions at the wrong times. A big thank-you also goes to everyone who's ever given me moths or caterpillars, especially Jenn Mongrain for all her intensive (and sometimes fearridden) labour last summer. I am also very grateful to Dr. Dan Janzen and Winnie Hallwachs and everyone at the Area de Conservación Guanacaste in Costa Rica for letting me come down and "torture" all the big caterpillars they worked so hard to find. Thank you for the high-speed drives down long winding roads and lending your expertise to my study. Thank you to Dr. Paul Martin and Vanya Rohwer for allowing me access to their yellow warblers and the facilities that housed them. And of course, thank you to Sarah Davis for investigating the first clicking caterpillar and starting this wonderful project.

I would also like to extend my gratitude to the members of my committee, Dr. Jean-François Landry and Dr. Tom Sherratt, for their comments and input, as well as for reading the tome that is my thesis. Thank you also to Dr. Jeff Dawson for answering any 
questions I had and for lending me the use of his tools as needed. Last, but never least, to Miss Canada, Dr. Jayne Yack, for getting me to do all of this in the first place. Thank you for welcoming me into your lab and then keeping me there, for challenging me and making things interesting, for never pretending not to be in your office (that I know of) and for ensuring that a caterpillar will never get past me without getting its bum pinched.

Finally I'd like to thank my family; to my parents for agreeing that it wasn't a bad idea to become a student again and to my sister for always wanting to help, but being too artsy to take advantage of. My most heartfelt thanks go to Snuffaluffagus, for supporting me no matter what, for putting up with all my drama while I was writing and for taking care of all the housework that I've been neglecting. And also to the cats, for waking me up in the mornings and making sure to lay on whatever I needed most.

Funding for this study was provided to Dr. J. E. Yack by the Natural Sciences and Engineering Research Council (NSERC) and the Canadian Foundation for Innovation (CFI). Funding to V. Bura was provided by Carleton University, the David and Rachel Epstein Foundation and NSERC (CGS-M). 


\section{TABLE OF CONTENTS}

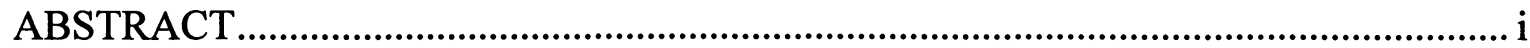

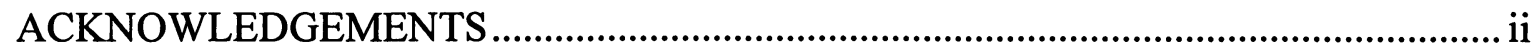

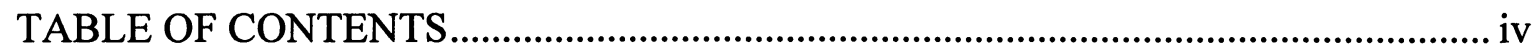

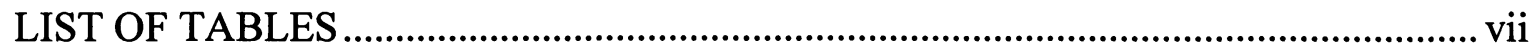

LIST OF FIGURES ................................................................................................

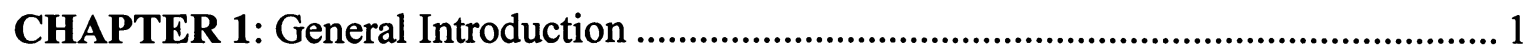

Acoustic communication in caterpillars....................................................... 2

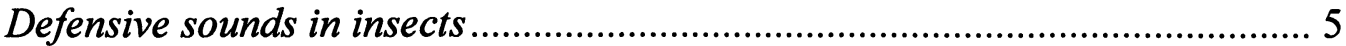

Thesis Objectives ....................................................................................... 11

CHAPTER 2: Defensive clicking in the tobacco hornworm, Manduca sexta (Bombycoidea: Sphingidae)........................................................................................ 12

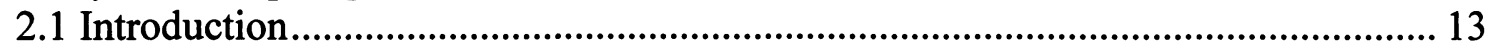

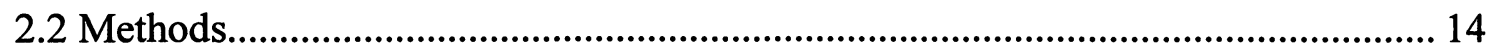

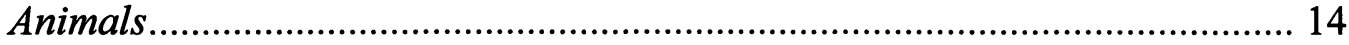

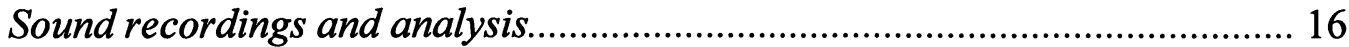

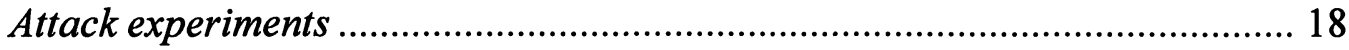

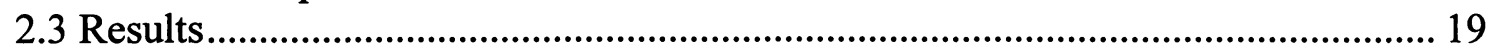

General behavioural observations ............................................................... 19

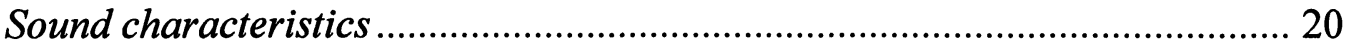

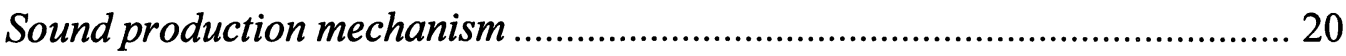

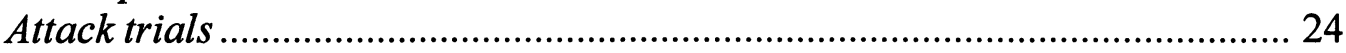

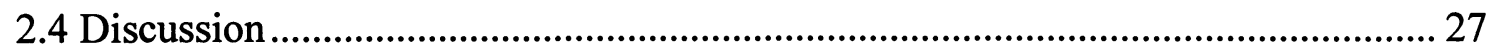

CHAPTER 3: Acoustic stridulatory signals in caterpillars of the great peacock moth

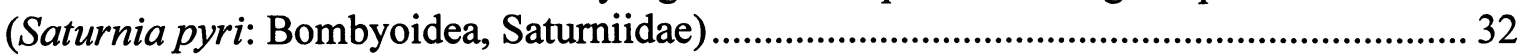

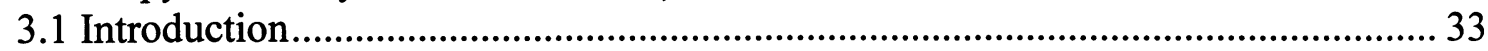

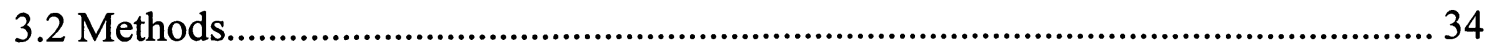

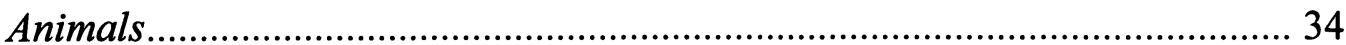

Sound production mechanism ....................................................................... 34

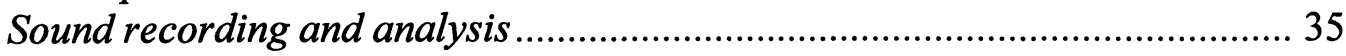

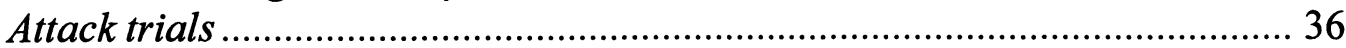

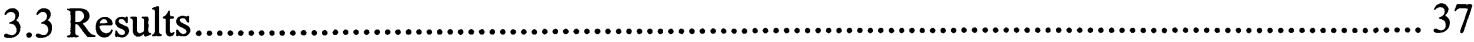

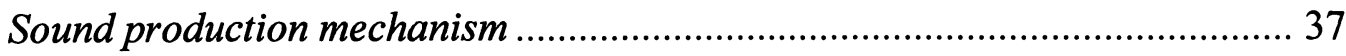

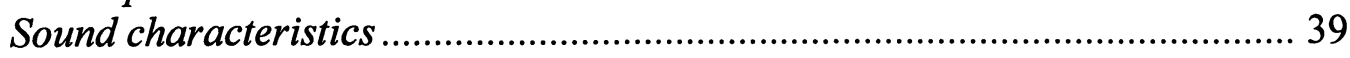

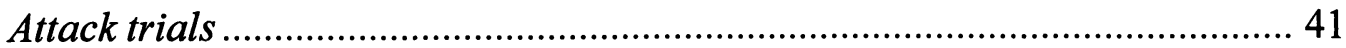

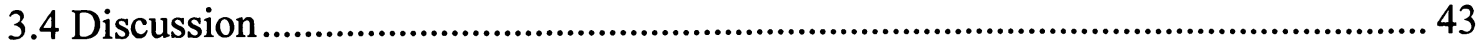

CHAPTER 4: Defensive whistles of the Walnut sphinx caterpillar, Amorpha juglandis

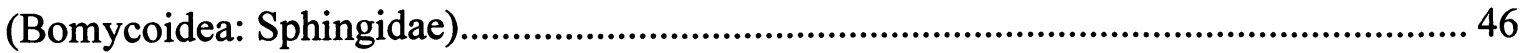

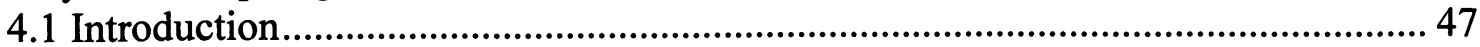

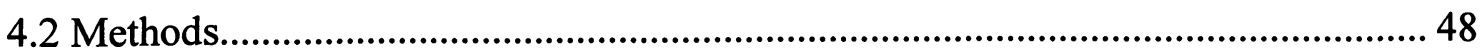

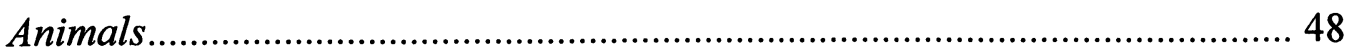




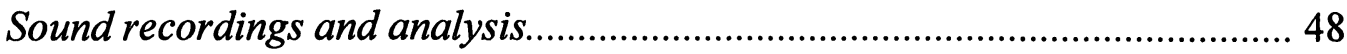

Sound production mechanism ....................................................................... 50

Anatomy of spiracles .................................................................................. 51

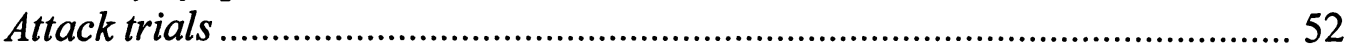

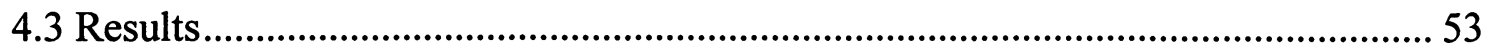

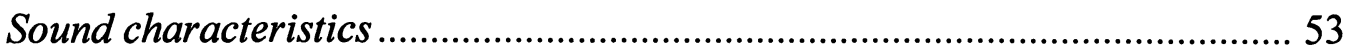

Sound production mechanism .................................................................... 57

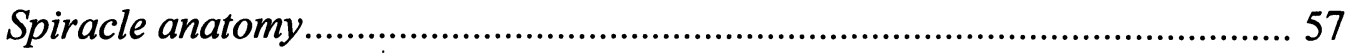

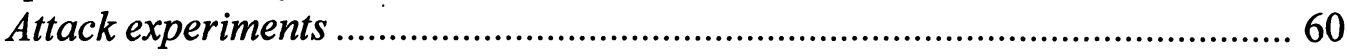

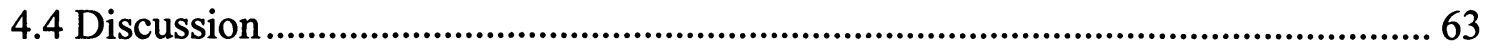

CHAPTER 5: Sound Production in Caterpillars: A comparative survey of defensive behaviours in Bombycoidea and other large caterpillars .....................................................6 69

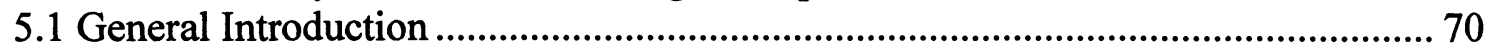

5.2 PART A: Survey of Sound Production and Associated Behaviours....................... 72

5.2.1 Introduction .................................................................................................. 72

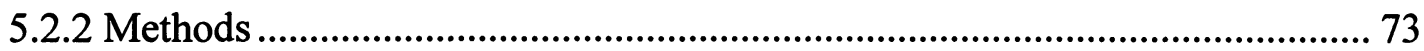

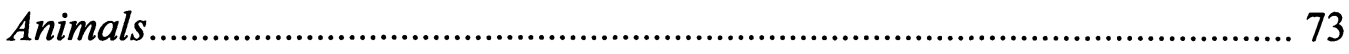

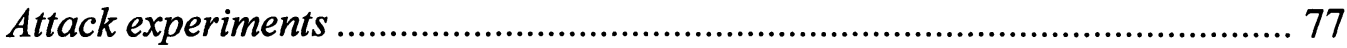

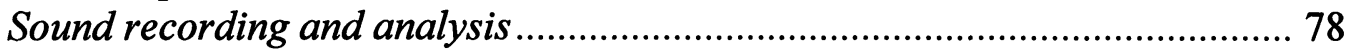

Head capsule and mandible morphology .................................................... 79

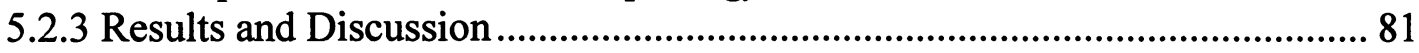

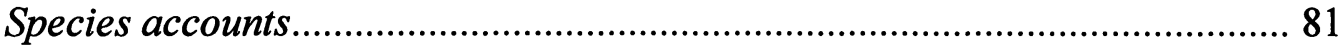

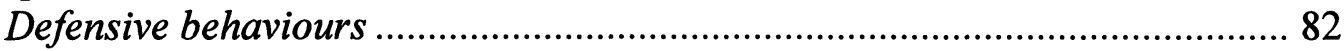

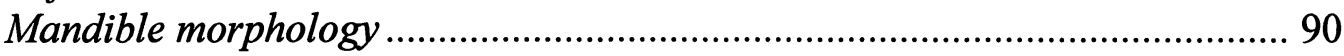

Sheets

FAMILY: BOMBYCIDAE

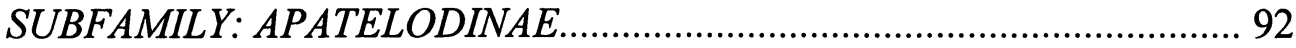

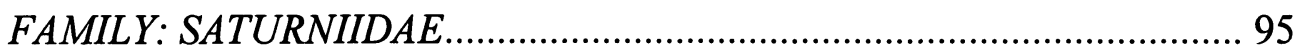

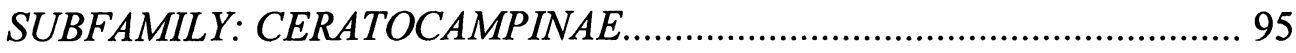

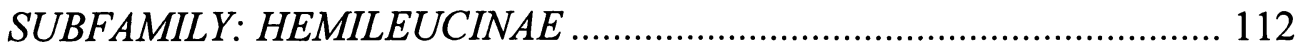

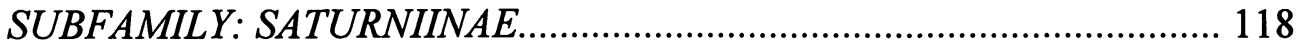

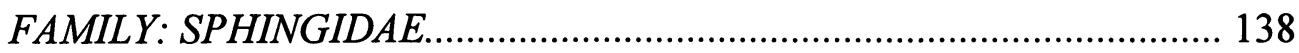

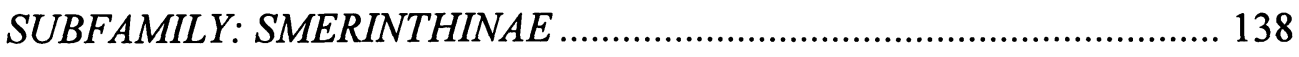

SUBFAMILY: SPHINGINAE ...................................................... 155

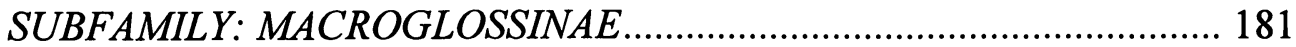

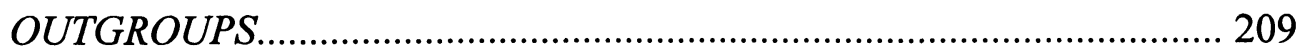

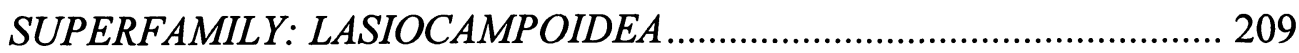

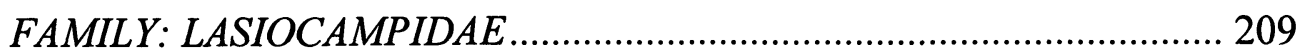

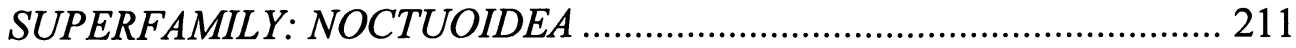

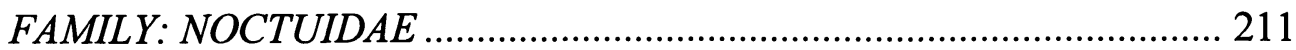

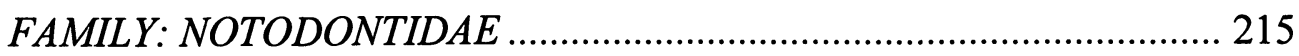

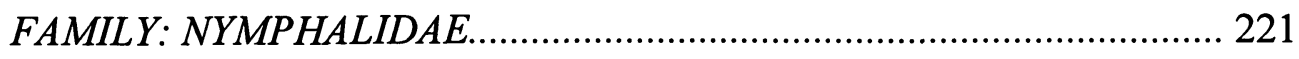

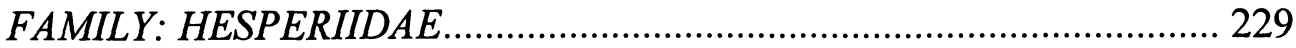

Taxonomic distribution of airborne sound production in Lepidoptera

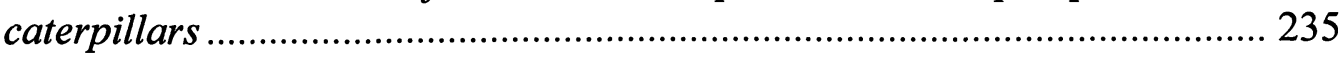


5.3 PART B: Evolutionary Origins of Communication signals ................................ 241

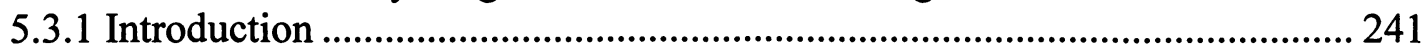

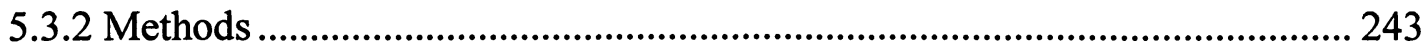

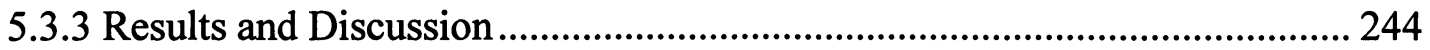

Proposed origins of mandible signals...................................................... 244

Proposed origins of spiracular sounds................................................. 245

Proposed origins of rasping sounds ..................................................... 248

5.4 PART C: Why do Some Caterpillars use Defensive Sounds? .............................. 252

5.4.1 Introduction .................................................................................................. 252

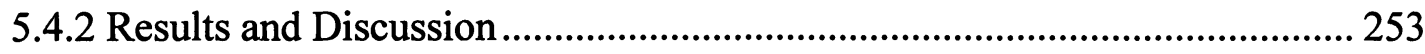

What is the function of airborne sound production in caterpillars? ........ 253

Why don't all caterpillars make defensive sounds?.................................. 264

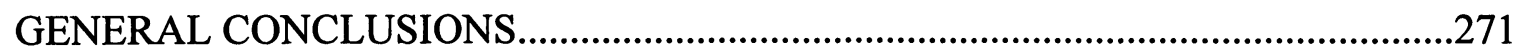

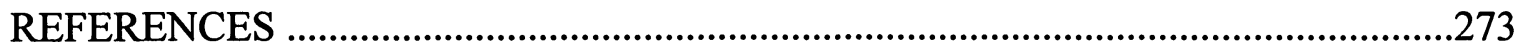

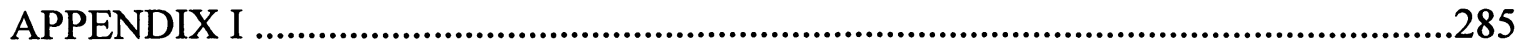




\section{LIST OF TABLES}

1.1 Summary table of current knowledge of sound production in Bombycoidea

caterpillars.

4.1 Spectral and temporal characteristics of sounds in A. juglandis.................................56

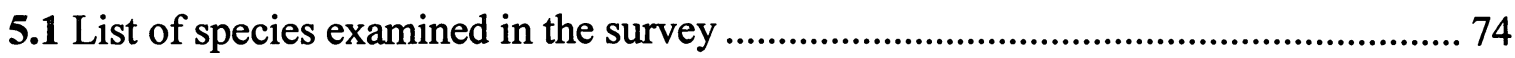

5.2 Summary of defensive behaviours and morphological features .............................. 231

5.3 Spectral and temporal characteristics of defensive sounds ..................................... 234

5.4 Specific behaviours observed in Bombycoidea caterpillars .................................... 254

5.5 Combinations of behaviours observed in Bombycoidea caterpillars........................ 255 


\section{LIST OF FIGURES}

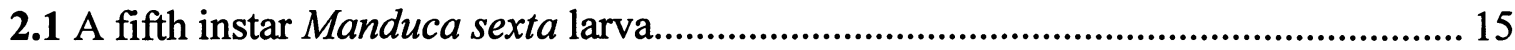

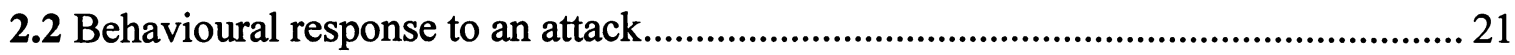

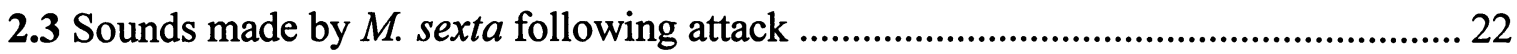

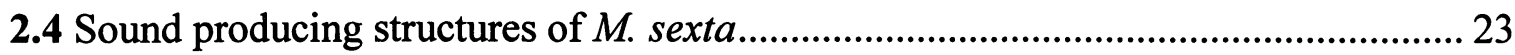

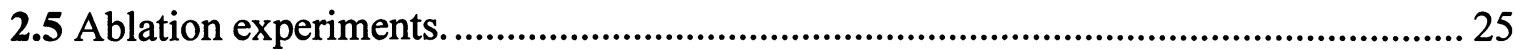

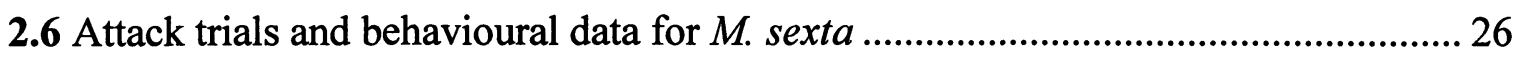

3.1 Morphology and sound producing structures of Saturnia pyri.................................. 38

3.2 Sounds made by $S$. pyri following attack................................................................... 40

3.3 Attack trials and behavioural data for $S$. pyri ............................................................ 42

4.1 Sounds made by Amorpha juglandis following attack. .............................................. 55

4.2 Body movements associated with sound production .................................................. 58

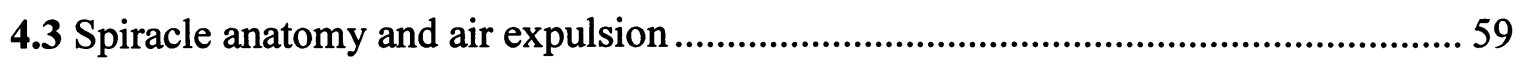

4.4 Reaction of a yellow warbler to the defensive sounds of $A$. juglandis ....................... 62

5.1 Representative trace from a sound producing caterpillar displaying characteristics

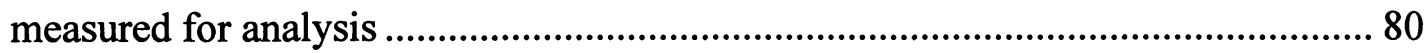

5.2 Topographic nomenclature of mandible morphology ................................................. 91

5.3 Olceclostera angelica ............................................................................................ 94

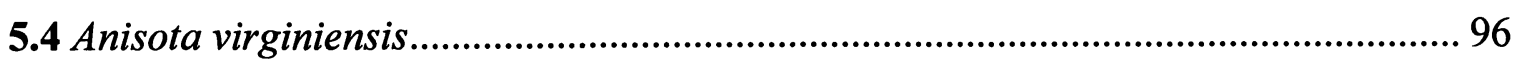

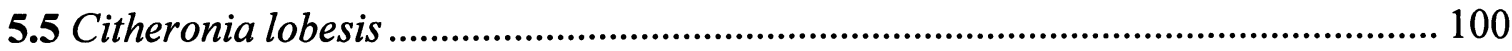

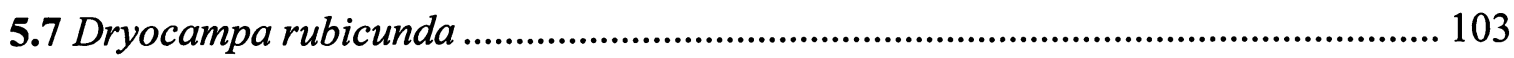

5.8 Eacles imperialis................................................................................................. 105

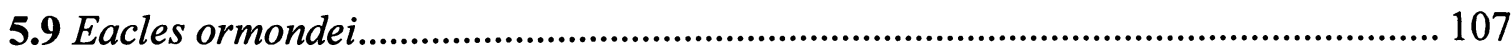


5.10 Schausiella santarosensis

5.11 Sounds produced by late instar $S$. santarosensis

5.12 Automeris postalbida

5.13 Automeris io

5.14 Automeris phrynon.

5.15 Actias luna 120

5.16 Sounds produced by late instar $A$. luna

5.17 Antheraea pernyi 123

5.18 Antheraea polyphemus. 126

5.19 Sounds produced by late instar A. polyphemus. 127

5.20 Attacus atlas 129

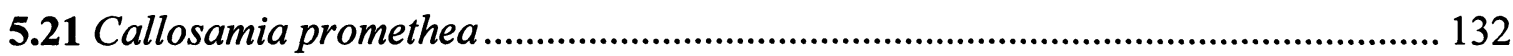

5.22 Sounds produced by late instar C. promethea …..................................................... 133

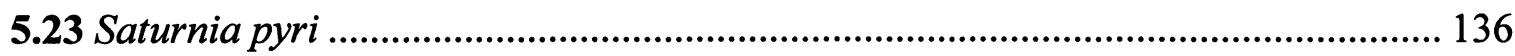

5.24 Sounds produced by late instar $S$. pyri............................................................... 137

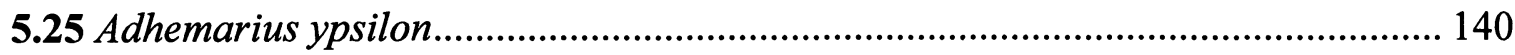

5.26 Amorpha juglandis............................................................................................... 143

5.27 Sounds produced by late instar Amorpha juglandis .............................................. 144

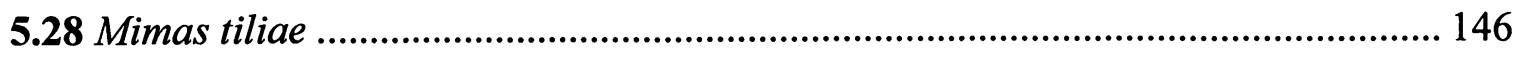

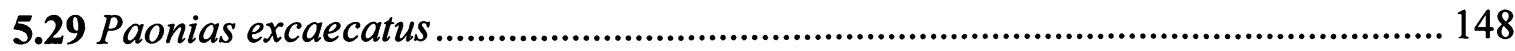

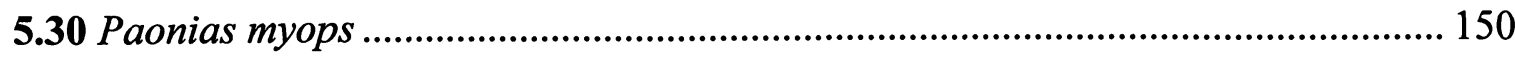

5.31 Smerinthus cerisyi .......................................................................................... 152

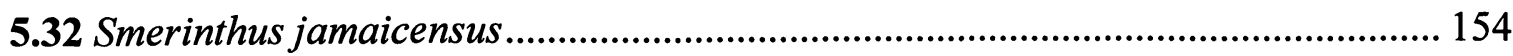




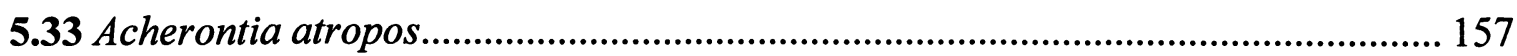

5.34 Sounds produced by late instar $A$. atropos.......................................................... 158

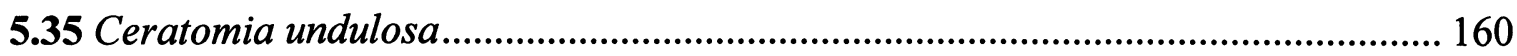

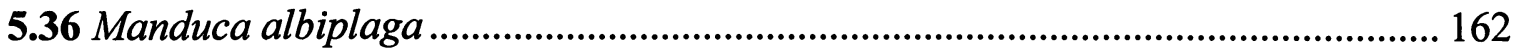

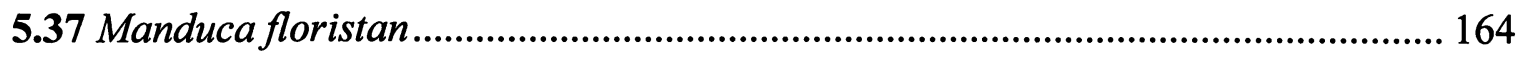

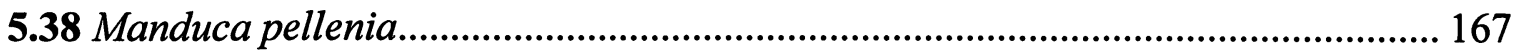

5.39 Sounds produced by late instar M. pellenia ……..................................................... 168

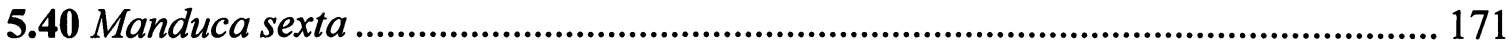

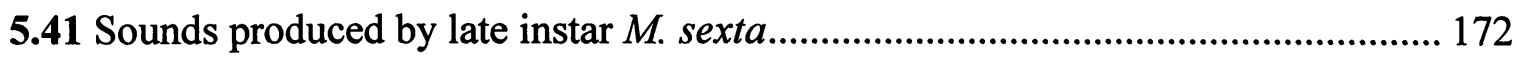

5.42 Sphinx chersis ..................................................................................................... 174

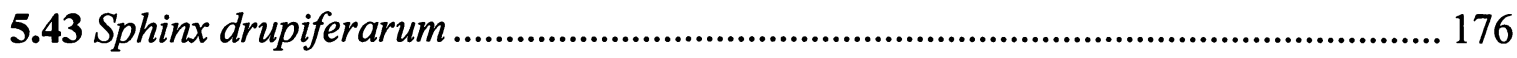

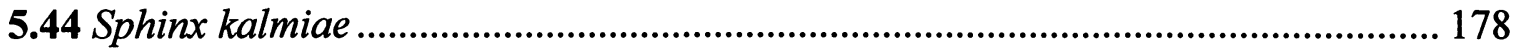

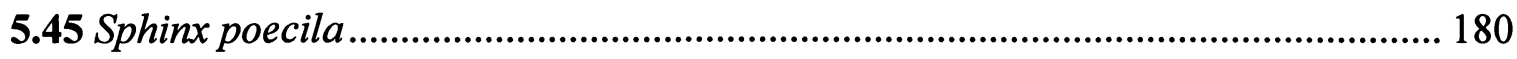

5.46 Darapsa choerilus............................................................................................... 182

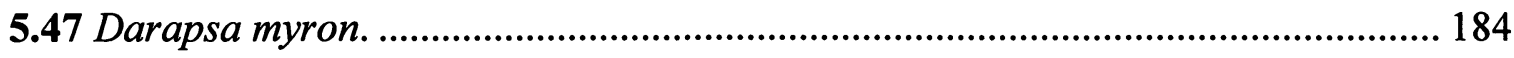

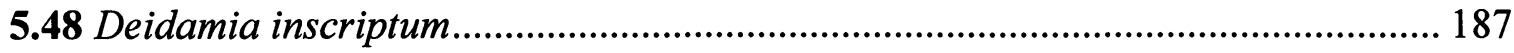

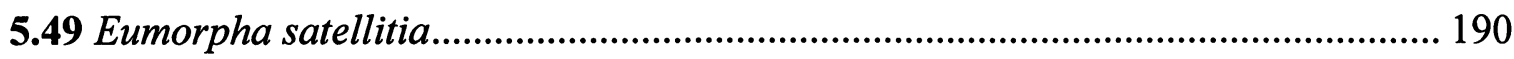

5.50 Sounds produced by late instar E. satellitia ......................................................... 191

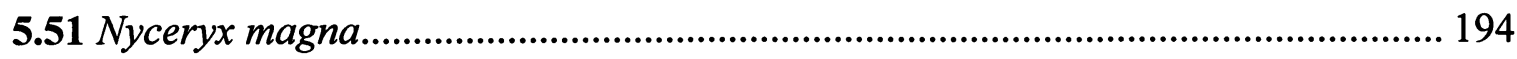

5.52 Sounds produced by late instar $N$. magna .............................................................. 195

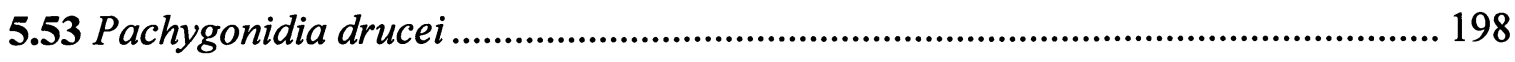

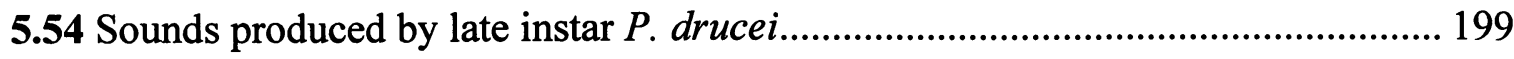

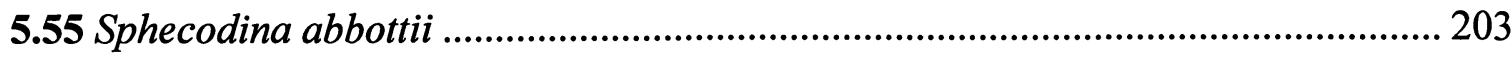


5.56 Sounds produced by late instar S. abbottii.

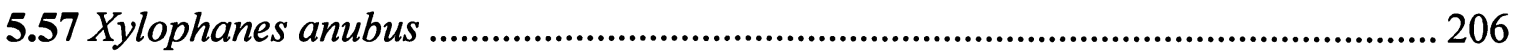

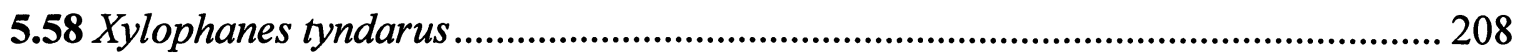

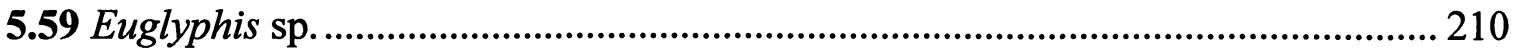

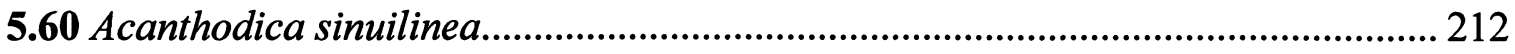

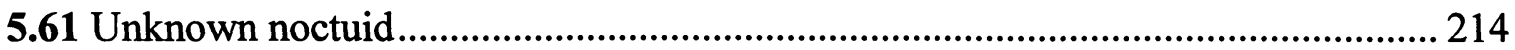

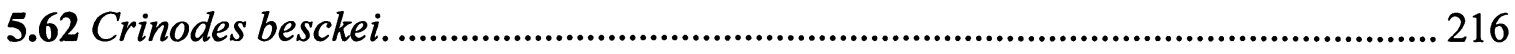

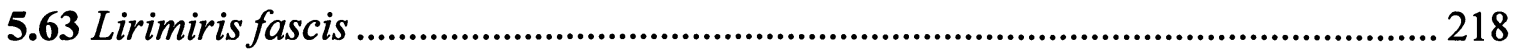

5.64 Lirimiris guatemalensis .................................................................................... 220

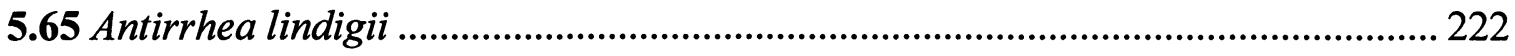

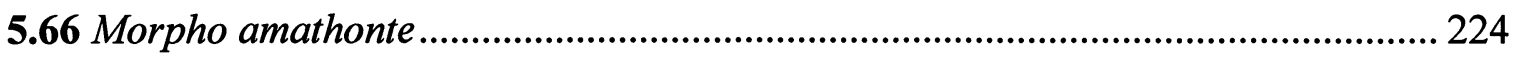

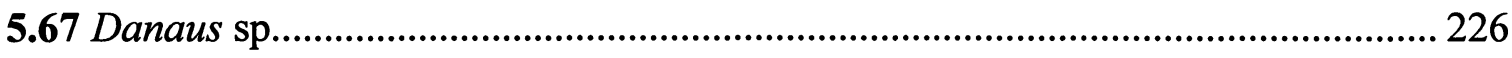

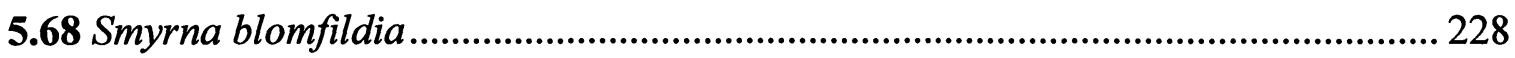

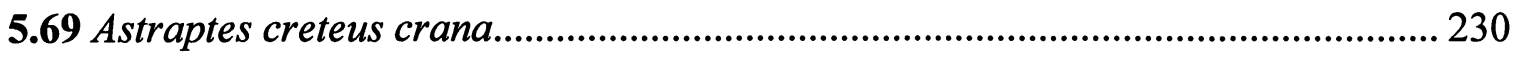

5.70 A phylogeny of the superfamilies of the Lepidoptera. ........................................... 236

5.71 Phylogeny of the Bombycoidea .............................................................................. 238

5.72 Phylogeny of the subfamilies of Saturniidae and Sphingidae ............................... 239

5.73 A hypothesis for the ritualization of incidental clicks to clicking and stridulation

5.74 Proposed evolution of air expulsion through the spiracles from twitching ........... 247

5.75 Comparison of sounds made by $S$. abbottii to those made by $A$. atropos.............. 250

5.76 Hypothesis for the evolution of the rasping mechanism from noisy regurgitation. 251 


\section{CHAPTER 1}

GENERAL INTRODUCTION 
Acoustic communication in Lepidoptera is a well documented phenomenon. The topic has been reviewed extensively (e.g. Spangler 1988, Scoble 1995, Conner 1999, Miller and Surlykke 2001, Minet and Surlykke 2003, Yack 2004, Yack and Hoy 2004) and has focused almost exclusively on adult Lepidoptera (moths and butterflies) that have evolved hearing organs multiple times for bat detection and, in a few cases, to communicate with mates. Sound production has also evolved multiple times, and shown to play roles in defence against bats and in courtship. Significantly less information is available on the role of acoustics in caterpillars, and in other holometabolous larvae in general, but abundant circumstantial references and some experimental studies suggest this mode of communication is, in fact, widespread.

\section{Acoustic communication in caterpillars}

Most formal reviews of acoustic communication in Lepidoptera do not include discussion of larval acoustics, yet, more recently, an increasing number of studies have been published on the topic. The lack of existing evidence for the use of acoustics in caterpillar communication may be due in part to the lack of technological advancements necessary to study these systems (e.g. laser vibrometry, highly sensitive and ultrasound detecting microphones). With these advancements, however, it is becoming more and more apparent that caterpillars use both substrate-borne (vibrations) and air-borne signals to detect and communicate with conspecifics as well as predators and parasitoids. Vibrational signals are used in some families for territorial disputes (Drepanidae, Yack et al. 2001, Bowen et al. 2008, Scott et al. 2010a,b; Gracillariidae, Fletcher et al. 2006), mutualistic behaviours with ants (Riodinidae and Lycaenidae, Devries 1990, Travassos 
and Pierce 2000) and detection of predators or parasitoids (Gracillariidae, Bacher et al. 1996, Casas et al. 1998; Geometridae, Castellanos and Barbosa 2006; Heliozelidae, Low 2008). Evidence that lepidopteran larvae are using airborne communication is also increasing. Some caterpillars are capable of perceiving near-field airborne sounds through the use of filiform sensilla that are sensitive to particle displacement and function in the detection of the wing-beat sounds produced by approaching predators and parasitoids (Minnich 1936, Tautz and Markl 1978, reviewed in Taylor 2009). Sound production in caterpillars has been less well researched, although there are numerous anecdotal reports of silk and hawkmoth (Bombycoidea) caterpillars making a variety of noises, variously described as "singing" (Reed 1868), "the crack of an electric spark" (Bethune 1868), "tcêp or tceep" (Sanborn 1868) and "crackling-rasping" (Heinrich 1979). Based on anecdotal reports from the literature, and a preliminary survey by myself, Brown (2006) and Brown et al. (2007) (see Table 1.1 for a summary), it is presumed that sound production in Bombycoidea caterpillars is taxonomically widespread, and variable with respect to sound production mechanisms and function. The majority of the sounds previously mentioned in the literature have not been experimentally validated or characterized, and many questions remain about their taxonomic distribution, mechanism, and function.

My thesis will survey several species of Bombycoidea caterpillars, as well as selected outgroup species, for the occurrence of sound production. Where possible, I will explore the functions and mechanisms of these sounds, and how sounds are associated with other defensive behaviours. This information will be used to develop hypotheses on the non-signalling origins of sound production, and selection pressures that may explain 
Table 1.1 Summary of Bombycoidea caterpillars noted to make sounds (prior to my study).

\begin{tabular}{|c|c|c|c|c|}
\hline Family & Subfamily & Species & Sound Production? & Reference(s) \\
\hline Bombycidae & Bombycinae & Bombyx mori Linnaeus & No & Brown (2006), Brown et al. (2007) \\
\hline \multirow[t]{7}{*}{ Saturniidae } & Ceratocampinae & Dryocampa rubicunda Fabricius & No & Brown (2006), Brown et al. (2007) \\
\hline & Hemilucinae & Automeris io Fabricius & No & Brown (2006), Brown et al. (2007) \\
\hline & Saturniinae & Actias luna Linnaeus & Yes & Brown (2006), Brown et al. (2007) \\
\hline & & Antheraea polyphemus Cramer & Yes & Brown (2006), Brown et al. (2007) \\
\hline & & Attacus atlas & No & Yack lab, unpublished \\
\hline & & Hyalophora columbia (SI Smith) & No & Yack lab, unpublished \\
\hline & & Rhodia fugax Butler & Yes & Packard (1904), Dumortier (1963) \\
\hline \multirow[t]{8}{*}{ Sphingidae } & Smerinthinae & Amorpha juglandis Smith $^{\Gamma}$ & Yes & Sanborn (1868), Wagner (2005) \\
\hline & & Mimas tiliae Linnaeus & No & Brown (2006), Brown et al. (2007) \\
\hline & & Pachysphinx modesta Harris & No & Brown (2006), Brown et al. (2007) \\
\hline & & Smerinthus jamaicensis Drury & No & Brown (2006), Brown et al. (2007) \\
\hline & Sphinginae & Acherontia atropos Linnaeus ${ }^{1}$ & Yes & Mead (1869), Pearce (1886) \\
\hline & & Manduca sexta Linnaeus & Yes & Brown (2006), Brown et al. (2007) \\
\hline & Macroglossinae & Hyles euphorbiae Linnaeus & No & Brown (2006), Brown et al. (2007) \\
\hline & & Sphecodina abbottii Swainson $^{1}$ & Yes & Dumortier (1963), Heinrich (1979), Wagner (2005) \\
\hline
\end{tabular}

${ }^{1}$ Information from literature only 
the diversity of sound production in Lepidoptera. The remainder of this general introduction will discuss the literature on the function of sounds in insects.

\section{Defensive sounds in insects}

Acoustic communication is widespread in the class Insecta. Ears have evolved independently in many orders and function primarily in mate location/recognition and predator/parasitoid detection (reviewed in Hoy and Robert 1996, Field and Matheson 1998, Yager 1999, Stumpner and von Helversen 2001, Yack 2004, Yack and Dawson 2008). Acoustic signalling is also prevalent, occurring in many orders in the form of vibrations transmitted through air, water, or solid substrates. In some cases, sounds and vibrations are directed at conspecifics and function in social contexts, like courtship and mating (e.g. courtship songs in crickets, cicadas, lacewings, etc., Bailey 2003; moths, Conner 1999), territorial interactions (e.g. caterpillars, Yack et al. 2001, Fletcher et al. 2006, Bowen et al. 2008; crickets, Mason 1996; larval caddisflies, Jansson and Vuoristo 1979), foraging (e.g. ants, Roces and Hölldobler 1996) and group cohesion (e.g. sawflies, Fletcher 2007). In other cases acoustic signals are directed at predators, triggered by disturbance and are considered to be defensive in nature. Disturbance signals are spectrally broadband and temporally simple so they can be perceived by a large number of predators (Masters 1980). Some examples include, the airborne sounds produced by some beetles, wasps and mantids, which are generally considered defensive signals (Edmunds 1974, Masters 1979, 1980). Despite the prevalence of such defensive sounds in insects, their function has been little explored compared to those associated with conspecific communication. These sounds have been proposed to function in startle, 
acoustic aposematism or warning, jamming, mimicry, alarm or recruitment and unprofitability.

Airborne sounds of Bombycoidea caterpillars likely function as defensive sounds since to the best of my knowledge, these caterpillars do not possess ears capable of hearing these airborne sounds and are therefore unlikely to function in conspecific communication (Yack 2004), and because in most reports where the stimulus for initiating the sound was mentioned, sounds were triggered by some sort of attack or disturbance (e.g. Reed 1868, Sanborn 1868, Federley 1905, Brown et al. 2007). Therefore the following literature review will focus on defensive sounds in insects. Below I will outline the classic categories for the function of defensive signals, however, it is important to note that these divisions may not be mutually exclusive.

\section{Startle}

Startle displays, also known as 'deimatic' displays, consist of a prey animal responding to an attack by confronting the predator with a set of behaviours that have been suggested to frighten or intimidate (Edmunds 1974). Startle displays have three features that are generally agreed upon: they should be used only after the prey animal has been discovered; they should be conspicuous and cause the predator to hesitate momentarily, giving the prey an opportunity to escape; and predators should habituate to the signals as the prey are otherwise undefended (Edmunds 1974, Sargent 1990, Ruxton et al. 2004). Such displays usually consist of a behaviour that would otherwise be unexpected, such as a sudden flash of bright colours, or the emission of a loud noise. Sounds are often involved in startle displays (Edmunds 1974), though their sole use may 
not be effective against all predators. The startle display of the palatable peacock butterfly, Inachis io, involves the use of both bright coloration and acoustics (Vallin et al. 2005). The visual aspect of the display has been shown to function well as an antipredator mechanism against birds, while the sounds did not (Vallin et al. 2005). However, the ultrasonic clicks produced by the butterflies were found to function effectively against bats (Mohl and Miller 1976), which would not have been influenced by the visual portion of the display. The clicks produced by both arctiid moths (reviewed in Conner 1999) and tiger beetles (Yager and Spangler 1997) in response to bat predation may also function as startle sounds in some cases, most notably when the encounter with such species is rare (Bates and Fenton 1990).

\section{Acoustic aposematism/Warning}

The term 'aposematism' is typically associated with visual warning colours that an animal may possess to advertise its unprofitability (Poulton 1890), such as the classic examples of the yellow and black markings of many Hymenoptera warning of their potent stings or the red and black seven-spotted ladybird beetle whose coloration warns of its toxicity (Marples et al. 1994). Predators must learn the association between colour and unprofitability either by sampling the prey and rejecting it or though an innate response (Edmunds 1974). However, warning signals are not all communicated visually; some animals will also use odours and sounds to communicate their defences (Ruxton et al. 2004). These sounds may be associated with a chemical defence, such as the noxious secretions of clicking arctiid moths (reviewed in Conner 1999, Hristov and Conner 2005, Ratcliffe and Nydam 2008), tiger beetles (Yager and Spangler 1997) and stridulating 
sand beetles (Masters 1979); a potent sting from a hymenopteran such as a stridulating velvet ant (Masters 1979) or a hissing bumble bee (Kirchner and Röschard 1999); or a mechanical defence like the sharp spine of a stridulating water beetle (Masters 1979). As with the visual signals mentioned above, after the association between the sound and defence is learned, the sound alone should be sufficient to deter predation (e.g. Hristov and Conner 2005).

\section{Enhanced learning}

A prey animal's defensive display may consist of more than one component, known as multimodal signalling. One hypothesis explaining the existence of multimodal displays is that of enhanced learning (reviewed in Rowe and Guilford 1999).

Conspicuously coloured animals may also produce odours or sounds that can function to enhance the learned association between the animal's colour and its distastefulness or chemical defence. With respect to sound, two studies have shown that domestic chicks learn to associate colour to distastefulness more rapidly when either the buzzing of a bumble bee or a pure tone is played while they are foraging (Rowe and Guilford 1999, Rowe 2002). However, another study demonstrated no such association and suggested that the type of sound may play a factor regarding its effectiveness (Hauglund et al. 2006).

\section{Jamming}

The jamming hypothesis concerns the emission of prey sounds interfering with the sonar of hunting bats (reviewed in Conner 1999). Bats emit echolocation calls as they 
search for prey. Ultrasonic clicks are thought to 'jam' the echolocation calls of the bats and decrease the predator's ability to locate prey in two ways; by diminishing the bats' sense of distance or by convincing the bat that the prey is actually a non-existent object by feigning echoes (reviewed in Conner 1999). The predictions of this hypothesis state that attacks should be deterred immediately and that this behaviour should persist over time. Arctiid moths, which possess ultrasound-sensitive ears, are able to perceive the calls of bats and some species can initiate this anti-predator behaviour once they know they have been spotted (e.g. Corcoran et al. 2009).

Mimicry

Mimicry is the similarity of one animal to another, for the purpose gaining protection from predators (Müllerian and Batesian, Ruxton et al. 2004). It is a well studied phenomenon in the visual realm (see examples in Edmunds 1974) but it is not restricted to this modality. Two types of mimicry complexes that exist for visual defences can also be applied to acoustic defences. Protection from predators through acoustic mimicry can be obtained by palatable or undefended species that can produce sounds that are similar to those of defended species (Batesian) or by two or more defended species producing similar warning sounds that can enhance their effectiveness (Müllerian). For example, some palatable arctiid moths gain protection from predacious bats by mimicking the clicks produced by noxious species, while different noxious species themselves sound alike (Barber and Conner 2007). Also, many hoverflies and drone flies sound similar (if not exact) to their hymenopteran models, as well as very similar to each other (Brower and Brower 1965, Rashed et al. 2009). For a mimicry complex to persist 
the mimic and the model should overlap in range and if the model disappears, the mimic should no longer experience the benefits of the signal (Edmunds 1974).

\section{Alarm/recruitment of con/heterospecifics}

Alarm and recruitment sounds can be found in social insects or between insects that are involved in a mutualiatic relationship. These sounds alert others to a potential threat and gather more individuals to overcome a potential predator. To be successful, other members of the group should be capable of perceiving the sounds as well as living close enough to respond in a timely manner. For example, the hissing sounds of some honey bees, transmitted as vibrations, recruit other colony members to fight off hive intruders or predators like wasps (Sen Sarma et al. 2002, Papachristoforou et al. 2008). Recruitment calls can also be interspecific. Certain caterpillar and treehopper species will signal to their mutualistic partners, ants, calling out for aid in defending against predators and parasites (Travasso and Pierce 2000, Morales et al. 2008).

\section{Unprofitablility}

In addition to the above mentioned specific functions, these defensive sounds may also share the same function, to signal, "I am unprofitable". Prey species may simply be advertising to predators that they are well defended and will take a lot of energy to acquire as a food source, so the predator should look elsewhere. Though I am unaware of examples in the class Insecta, there are some in vertebrates (reviewed in Ruxton et al. 2004), including the classic example of stotting by gazelles which has been suggested to 
function to inform predators that the prey animal is very fast and will outrun them in a chase.

\section{Thesis Objectives}

The main goals of my thesis are to provide insight into which caterpillars produce sounds, the mechanisms they use to do so, and to test and develop hypotheses on the function and evolutionary origins of sound production. Toward this end, my specific objectives are to: (1) Test as many Bombycoidea species as possible for sound production and other defensive behaviours from various worldwide sources, including Europe, North American and Costa Rica. This will provide information on the taxonomic distribution of sound production and give insights to its potential evolutionary origins. (2) Characterize sounds whenever possible and determine their mechanism of production. This will provide information on the function of the signals, what predators might be listening to these sounds and how they may have developed from non-signalling behaviours. (3) To experimentally test the function of the sounds whenever possible to help determine their role in the defensive strategy of these caterpillars. In some cases where sound production in a caterpillar offered insights into a novel mechanism or function, and, when sufficient number of this species could be reared to conduct experimental trials, more details could be provided, and these species (Manduca sexta, Saturnia pyri and Amorpha juglandis) are described in more detail in Chapters 2, 3 and 4 respectively. Chapter 5 includes a survey of all species tested, including outgroups that represented other large caterpillars, as well as the taxonomic distribution of sound production and discussion on the evolutionary origins of sound production mechanisms in this group. 


\section{CHAPTER 2}

\section{Defensive clicking in the tobacco hornworm, Manduca sexta (Bombycoidea: Sphingidae)*}

*Parts of this chapter are being prepared for publication.

Manuscript: Bura, VL, Hnain A, Hick, J, Yack JE. Defensive clicking in the tobacco hornworm, Manduca sexta (in preparation for submission to Journal of Ethology in July, 2010)

Statement on contribution: V. Bura collected and analysed the data, prepared the figures and wrote the paper; A. Hnain collected some attack trial data and high sample recordings; J. Hick collected the data for the ablations (supervised by V. Bura) 


\subsection{Introduction}

Manduca sexta is one of the most widely studied insects with many reports on its biology, including studies of the endocrine system (e.g. Asuncion-Uchi et al. 2010), respiratory system (e.g. Greenlee and Harrison 2005) and pest management strategies (e.g. Gulbitti-Onarici et al. 2009). This species is widely used because of its large size, accessibility and ease of rearing. Given the interest in the caterpillar as a model laboratory species, it is surprising that until recently no record of its ability to make sounds had been documented.

Sound production was formally reported in $M$. sexta caterpillars for the first time only 3 years ago, by Brown et al. (2007). In that study, which focused on sound production in another caterpillar (Antheraea polyphemus), M. sexta was tested for sound production, and clicking sounds were reported in response to forcep attacks. Brown et al. (2007) also performed a small number of trials to show that sound production may be associated with defence, and specifically, as a warning of an impending regurgitant defence. It is surprising that in a previous study devoted to documenting the defensive behaviours of $M$. sexta (Walters et al. 2001) there was no mention of sound production. In that study, the authors stimulated the larvae by delivering a pinch using forceps or a poke with a nylon filament to the prolegs of the animal and recorded their reactions on videotape. Although these authors reported directed thrashing, opening the mandibles (biting) and regurgitation as responses of the caterpillars, sound was never mentioned. Therefore, either the sounds recorded by Brown were an anomaly or they were too quiet 
for Walters et al. (2001) to hear. Regardless, the phenomenon of sound production in $M$. sexta caterpillars requires further examination.

The purpose of this chapter is to further study the phenomenon in different populations of $M$. sexta caterpillars, to test the hypothesis that sounds are produced using the mandibles and to test the hypothesis proposed by Brown that these sounds function in acoustic aposematism. My specific objectives are to: (a) examine sound production in $M$. sexta from two additional geographic sources to determine if they are indeed making sounds; (b) to characterize the conditions that evoke sound production; (c) to characterize the acoustic properties of these sounds; and (d) to test the hypothesis that these sounds likely function as aposematic warning signals. This hypothesis will be tested based on the following predictions: (1) increased attacks will result in an increased number of clicks; (2) clicking is associated with regurgitation; (3) clicking precedes regurgitation; and (4) regurgitation is a chemical defence.

\subsection{Methods}

\section{Animals}

Manduca sexta Linneaus larvae (Fig. 2.1) were purchased from Canadian Feeders (Windsor, ON, Canada) and LiveFood (Mercier, QC, Canada). Larvae were initially reared on an artificial diet provided by their company of origin, then transferred to potted datura (Datura inoxia), tobacco (Nicotiana tabacum), or tomato plants (Solanum lycopersicum) in mesh cages at least 24 hours before experimentation. Caterpillars were housed in an insect rearing facility at Carleton University. All trials were performed on larvae in their fifth instars. 


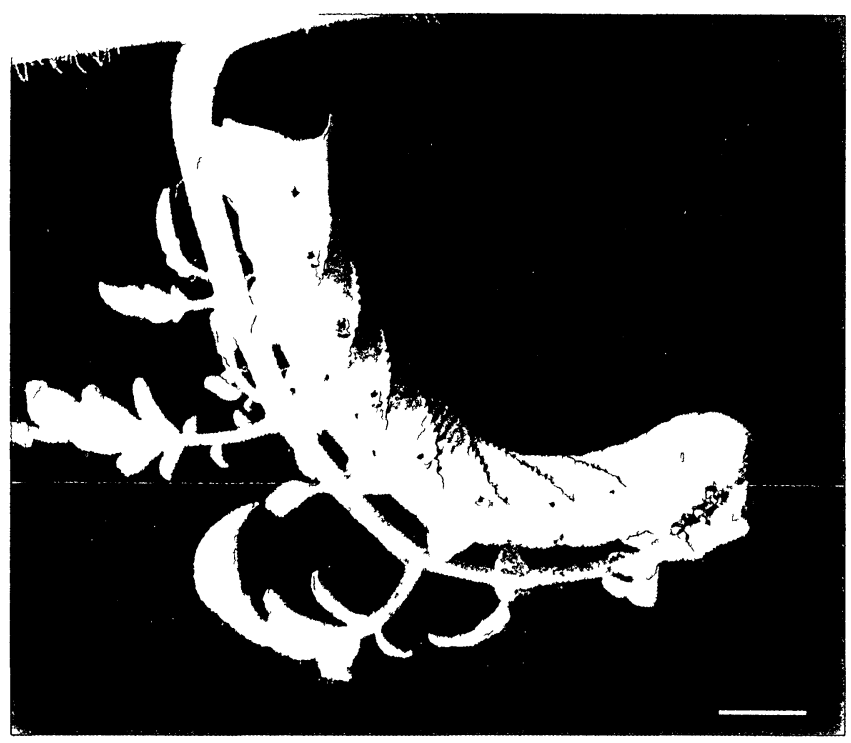

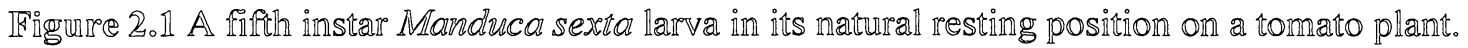
Scale bar $1 \mathrm{~cm}$. 


\section{Sound recordings and analysis}

Sounds analysed for spectral, intensity and temporal characteristics were recorded in an acoustic chamber (Eckel Industries Ltd., Cambridge, MA, USA) or an enclosure lined with acoustic foam, located at Carleton University. Caterpillars were placed on sprigs of hostplant and induced to signal using blunt forceps (see below). Sounds were analysed using Raven 1.2 Bioacoustics Research Program (Cornell Laboratory of Ornithology, Ithaca, NY, USA).

Temporal characteristics, including train duration, number of clicks per train, click duration and number of components in a click, were measured from sounds extracted as .wav files from video clips $(\mathrm{n}=16)$ of the attack trials (see below) using Quicktime 6.5.3. A train was defined as a string of clicks following an attack until sound production ceased and one click was defined as the smallest unit of sound distinguishable by the human ear (Broughton 1963).

Spectral characteristics were examined from five randomly chosen clicks from five animals. Sounds were recorded with a Brüel \& Kjær (Naerum, Denmark) 1/4" microphone type 4939 placed $10 \mathrm{~cm}$ from the head capsule of the larvae, amplified using a Brüel \& Kjær Nexus conditioning amplifier type 2690, and recorded onto a Fostex FR2 Field Memory Recorder (Gardena, CA, USA) at a sampling rate of $192 \mathrm{kHz}$. Spectra were produced using a 512-point Fast Fourier Transform (FFT) (Hann window).

For intensity characteristics, sounds were recorded at a distance of $10 \mathrm{~cm}$ using a Brüel \& Kjær 1/4" microphone type 4939 and amplitudes measured as voltages on a Tektronix THS720A oscilloscope. Voltages were converted to pascals $(\mathrm{Pa})$ based on the sensitivity output of the Nexus amplifier $(1 \mathrm{~V} / \mathrm{Pa})$ and subsequently transformed into $\mathrm{dB}$ 
SPL values using the following equation: Sound pressure level $(\mathrm{dB} \mathrm{SPL})=20 \log \left(\mathrm{P}_{\mathrm{i}} / \mathrm{P}_{\mathrm{o}}\right)$ where $P_{i}$ is the pressure in pascals and $P_{o}$ is $2 \times 10^{-5} \mathrm{~Pa}$ (threshold of human hearing at 1 $\mathrm{kHz})$.

Sound production mechanism

A previous study suggested that $M$. sexta caterpillars produce clicks using their mandibles (Brown et al. 2007). However this hypothesis was not confirmed experimentally, nor were details included on which parts of the mandibles may be involved specifically (for any clicking species). In the following experiments, this hypothesis was tested by documenting the anatomy of the mandibles and performing ablations to determine which parts were interacting.

Mandible anatomy and movements during sound production

Larval mouthparts were videotaped using a Sony HDR-HC7 HD Handycam (Tokyo, Japan) equipped with a Sony ECM-MS957 microphone and a macro lens. Videos were analyzed using iMovie 3.0.3 to determine if and how mandible movements were associated with sound production.

The anatomy of the mandibles was studied by dissecting pairs from sound producers, sputter coating with gold-palladium and examining using a JEOL JSM-6400 scanning electron microscope (Tokyo, Japan). Sets of ridges were defined according to their relative location on the mandible (outer face, anterior edge and inner face; see Results). 
Selected ablations

Note: This experiment was designed by me and carried out by an undergraduate student under my supervision. For the sake of completeness the data are included in this chapter.

To determine how mandibles interacted to produce clicking sounds, various sets of ablations were performed on different ridges on both of the mandibles. Larvae were anesthetised using carbon dioxide, and selected parts of the mandibles were ground down (i.e. removed) with a Vogue Professional 6700 nail drill (Woodland Hills, CA, USA) equipped with diamond dental burs. Three sets of experimental trials were performed; only inner ridges removed $(n=16)$, only outer ridges removed $(n=8)$, and only anterior ridges removed $(n=12)$. Control experiments were performed using a smooth drill bit that did not change the structure of the mandibles. Both pre-operation and post-operation attack trials were performed (see below) on each larva to test for sound production. The first 10 clicks of five 'outer' and six 'anterior' post-operation ablation trials were analyzed for changes in temporal characteristics (number of click components) using Raven.

\section{Attack experiments}

Attack trials were performed to determine the relationship between attack, sound production and other defences. Simulated attacks using methods similar to those in Walters et al. (2001) as well as those using blunt forceps were conducted. All trials were carried out on caterpillars resting on a sprig of hostplant that had been isolated for a minimum of 30 minutes prior to experimentation. Defensive behaviours were recorded 
using a Sony Mini-DV DCR-HC85 Handycam (Tokyo, Japan) and a Sony ECM-MS907 microphone placed 2-4 centimetres away from the heads of the larvae.

Attack trials simulating those in Walters et al. (2001)

Five pinches were delivered to the prolegs using sharp forceps, three to one side of the animal and two to the other, with approximately 3 seconds between each pinch (n $=10$ ). In an additional set of trials, five pokes to the prolegs were administered using a stiff piece of fishing line in the same manner $(n=5)$. Trials were analysed for the number of animals that produced sound and other accompanying behaviours.

Blunt forceps trials

Attack trials consisted of pinches being delivered to the head capsule of the larvae using blunt forceps. A pinch was delivered to the head capsule of the larva one, three or five times with approximately five seconds between consecutive pinches. Trials were analyzed in iMovie 3.0.3 to determine (i) the mean number of clicks in 60 s following one, three or five pinches, (ii) the occurrence of clicking and regurgitation, and (iii) the temporal relationship between the two.

\subsection{Results}

\section{General behavioural observations}

In general there are many ways to induce clicking in caterpillars of $M$. sexta including changing the food plant and poking the caterpillar, but the most reliable way to provoke signalling was to pinch the caterpillar with blunt forceps. This type of attack also 
induced other defensive behaviours including regurgitation, directed thrashing away from the site of attack and an inward curling of the head (Fig. 2.2) (relationships discussed below).

\section{Sound characteristics}

Larvae that produced sounds after being pinched once with forceps were used to determine the temporal characteristics of the clicks $(n=16)$. Trains ranged from 0.30 to 20.41 seconds (mean $3.49 \pm 4.92 \mathrm{~s}$ ) and contained an average of $6.69 \pm 8.93$ clicks per train (Fig. 2.3). Individual clicks lasted $45.08 \pm 29.09 \mathrm{~ms}$ on average and consisted of one to four components (mean $2.08 \pm 0.91, \mathrm{n}=25$, Fig. 2.3b). Although the clicks are audible to the human ear, spectral analysis revealed that the most energy is centered around 20-45 $\mathrm{kHz}$ (mean peak frequency 28.05 $\pm 9.85, \mathrm{n}=25$; Fig $2.3 \mathrm{~b}, \mathrm{c}$ ).

Sound levels were measured from 60 clicks obtained from five late instar larvae. Maximum amplitudes ranged from 82.2 to $93.9 \mathrm{~dB}$ SPL, measured at $10 \mathrm{~cm}$ from the source.

\section{Sound production mechanism}

Video analysis supports the hypothesis that clicks are produced using the mandibles. Scanning electron micrographs (Fig. 2.4) revealed the mandibles to be highly serrated and consisting of three main ridges: outer, anterior and inner. The interactions of the ridges on left and right mandibles produce the clicks as the mandibles are closed. I propose that when one mandible slides against the inner surface of the opposing mandible the outer and anterior ridges of one catches on the inner ridge of the other to produce 
a
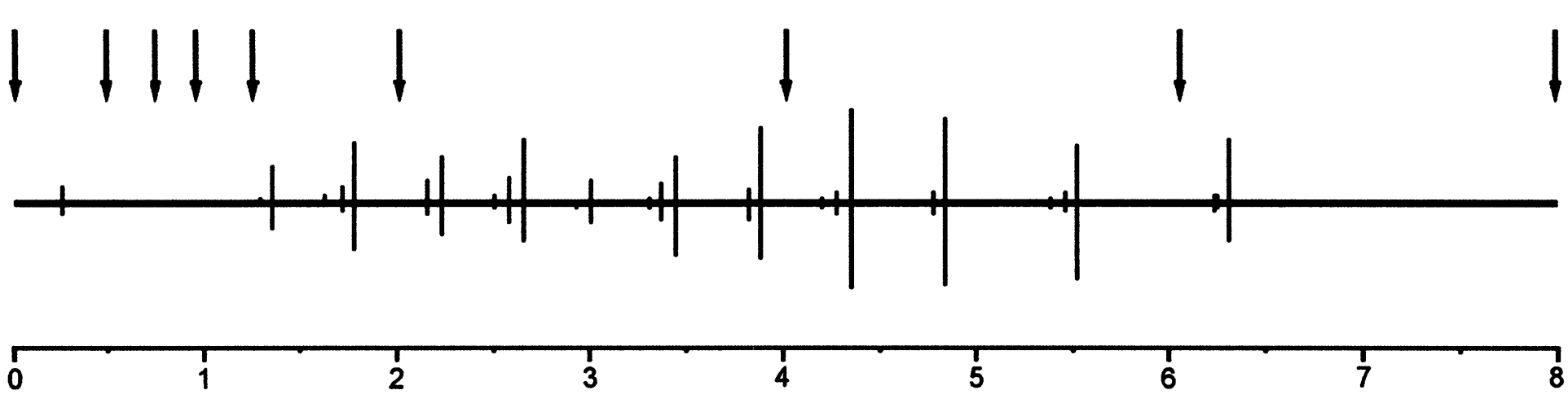

Time (s)

b

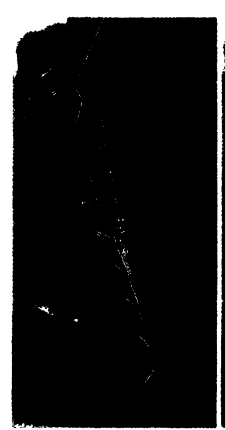

Pinch

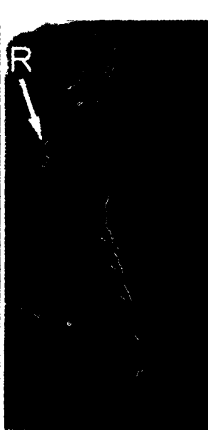

0.53

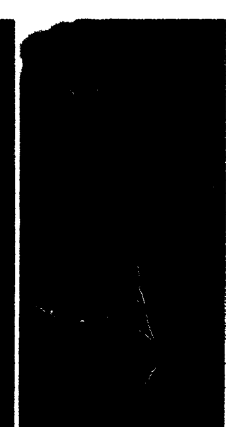

0.70

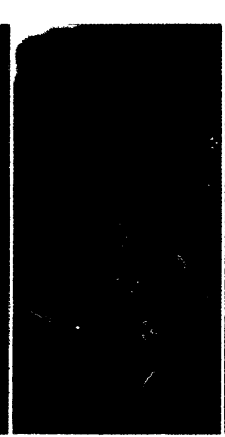

0.93

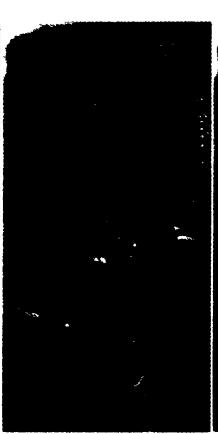

1.23

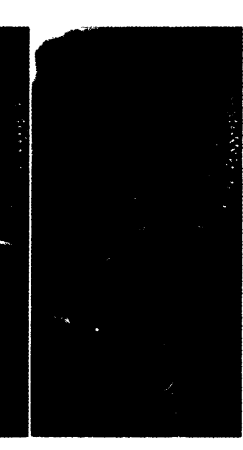

2.00

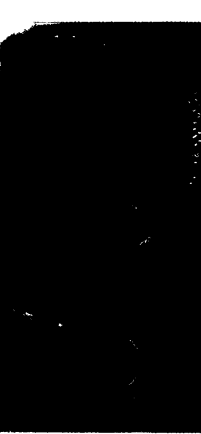

4.00

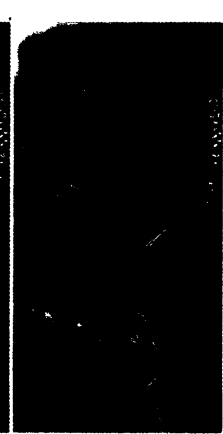

6.00

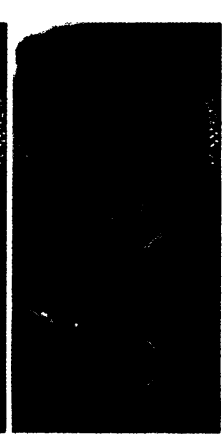

8.00

Time after pinch (s)

Figure 2.2 Behavioural response to an attack to the head region with forceps. (a) Oscillogram showing the click train produced by the larva during the first eight seconds following attack. Arrows correspond to video frames in b. (b) Video still frames showing typical defensive responses

(thrashing and regurgitation) following attack. Exact times of occurrence for each frame are indicated by black arrows in a. Regurgitant (R) can be seen in the second frame, indicated by the white arrow. 
a

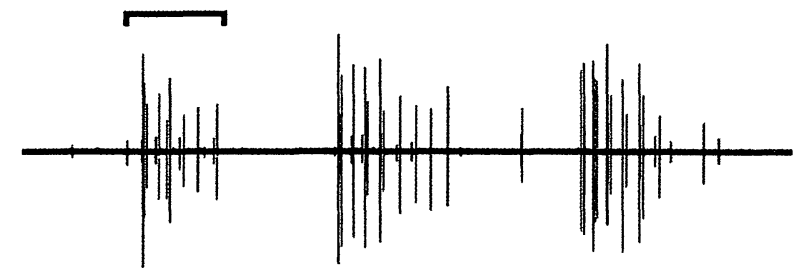

$\overline{1 s}$
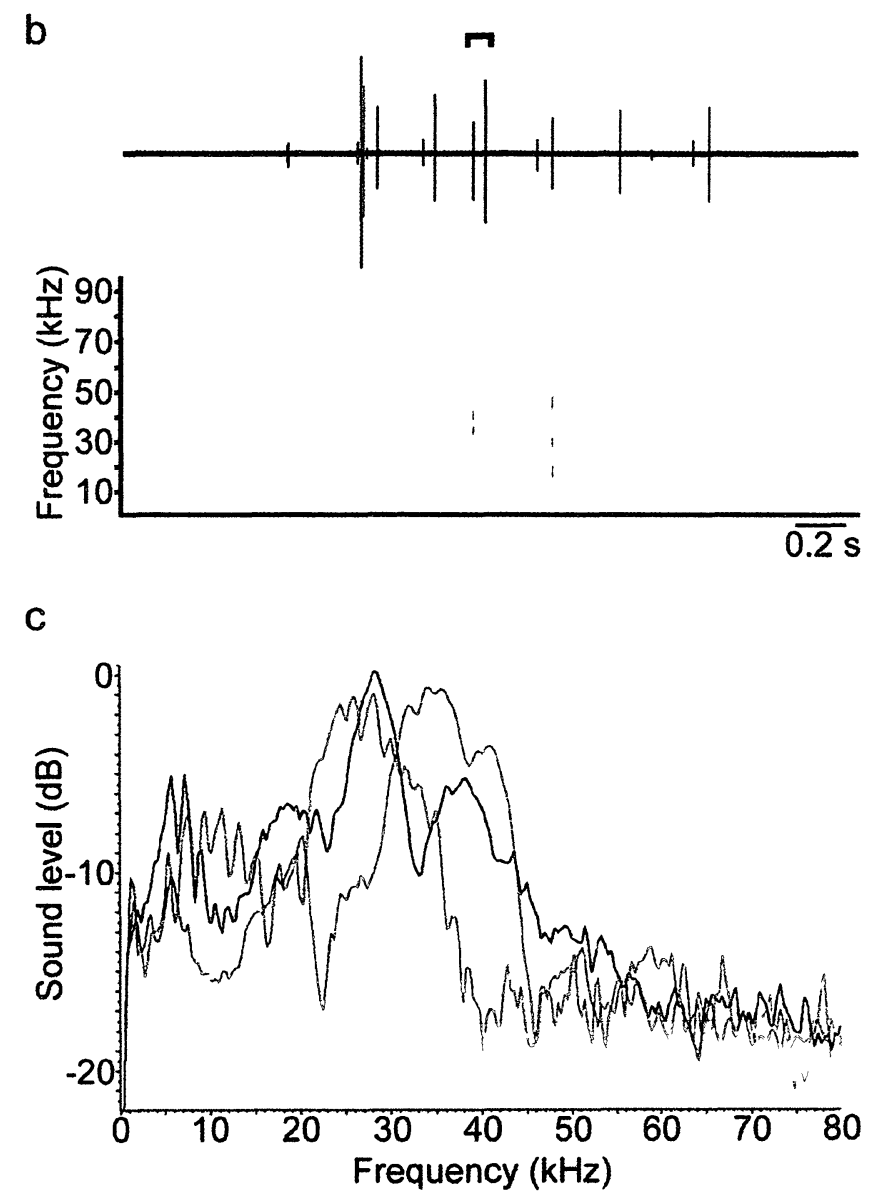

Figure 2.3 Sounds made by a fifth instar larva following attack. (a) Three click trains elicited by an attack to the head capsule. The bar outlines a click train expanded in b. (b) Expanded click train from (a) to show the individual components of the clicks (one click represented by smaller bar). The accompanying spectrogram illustrates the frequency range of each component click. (c) Power spectra of clicks from three different individuals showing that most energy occurs between 20-45 kHz. 


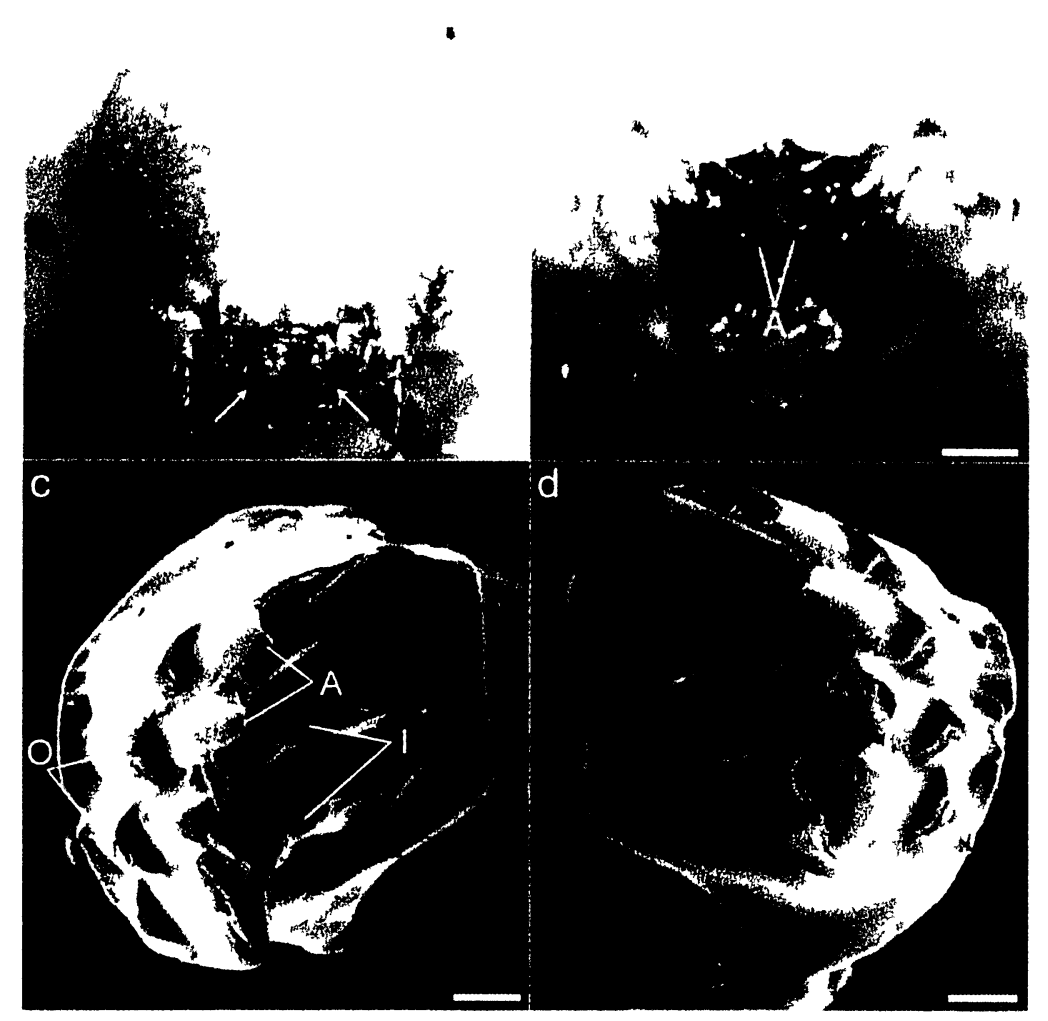

Figure 2.4 Sound producing structures of Manduca sexta. (a) Close up of the head region. Arrows indicate the location of the mandibles. Scale bar $1 \mathrm{~mm}$. (b) Close up of the mandibles, anterior ridges (A) are clearly visible. Scale bar $0.5 \mathrm{~mm}$. (c) Anterior view of the inner face of the right mandible showing the location of the different ridges, outer $(\mathrm{O})$, anterior $(\mathrm{A})$ and inner $(\mathrm{I})$. Scale bar $0.2 \mathrm{~mm}$. (d) Left mandible in the same orientation demonstrating that both mandibles are similarly structured. Scale bar $0.2 \mathrm{~mm}$. 
individual clicks. This is supported by data from the ablation experiments (Fig. 2.5).

When the inner ridges were ground down, sound production was eliminated in $87.5 \%$ of caterpillars $(n=16)$. Although grinding the other ridges did not consistently remove sound production, the temporal characteristics of the clicks were always changed. The number of click components differed between pre- and post-operation sound production. Prior to experimentation the majority of clicks contained two or more components (68\%). After both outer and anterior ridge ablations this number decreased to $20 \%$ and $15 \%$ respectively, and the number of one component clicks dominated ( 80 and $85 \%$ ). Control animals $(n=18)$ continued to click after experimentation with no change in temporal characteristics. Therefore, clicks are clearly produced using the mandibles and the inner ridges appear to be the most important for the production of clicks in this species.

Attack trials

Clicks were consistently produced by $M$. sexta when a pinch was delivered to the head capsule with blunt forceps. Sounds were also reliably produced when the caterpillars were stimulated in ways similar to those in Walters et al. (2001). Clicks were recorded in $80 \%$ of leg pinch trials $(n=10)$ and $60 \%$ of leg poke trials $(n=5)$. In contrast to the head-pinch trials, larvae in these trials readily bit the attacking forceps, also reported in Walters et al. (2001).

In general, both acoustic signalling and defensive regurgitation increased with the degree of disturbance (Fig. 2.6 a-c). In one-pinch trials $77 \%$ of larvae produced sound $(n=22)$. This increased to $85 \%$ in three-pinch trials $(n=20)$ and $100 \%$ in five-pinch trials 


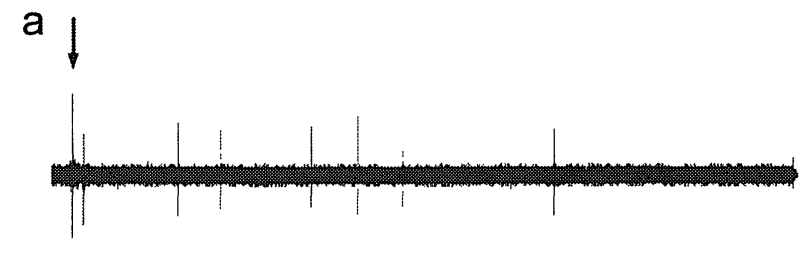

$\overline{0.5 \mathrm{~s}}$

b

$\overline{0.5 \mathrm{~s}}$

C

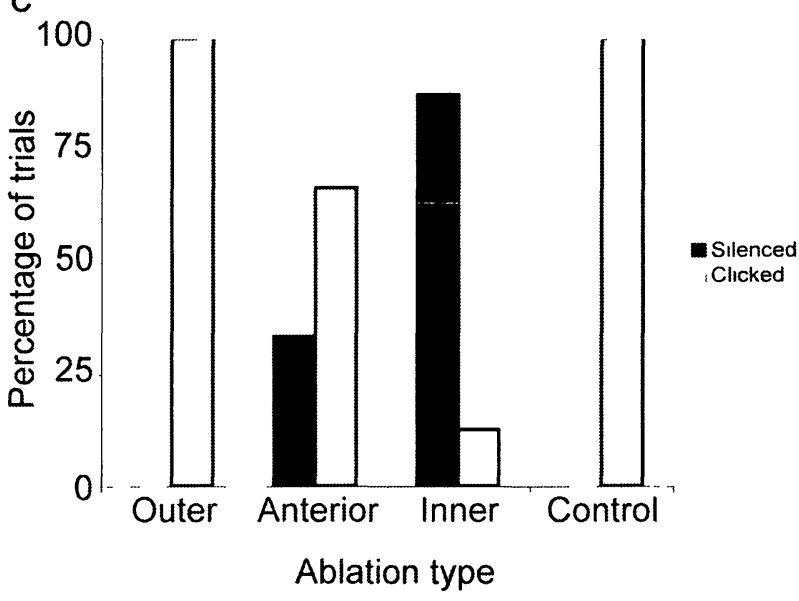

Figure 2.5 (a) Pre-operation oscillogram showing a click train following an attack (arrow). (b) Post-operation oscillogram of an inner ablation showing the removal of sound production following an attack (arrow). (c) Percentage of larvae that were silenced or continued to click following different ridge ablations. 
a

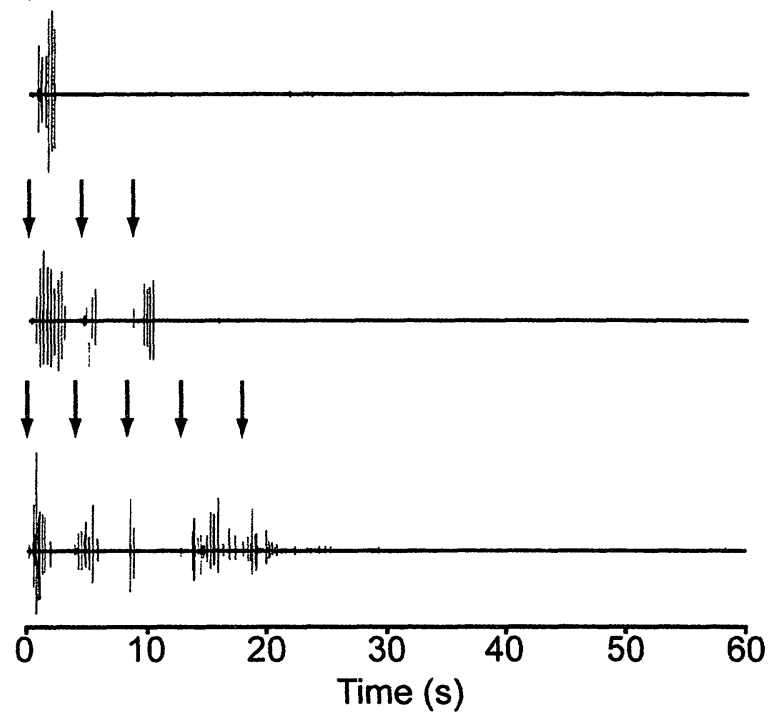

C

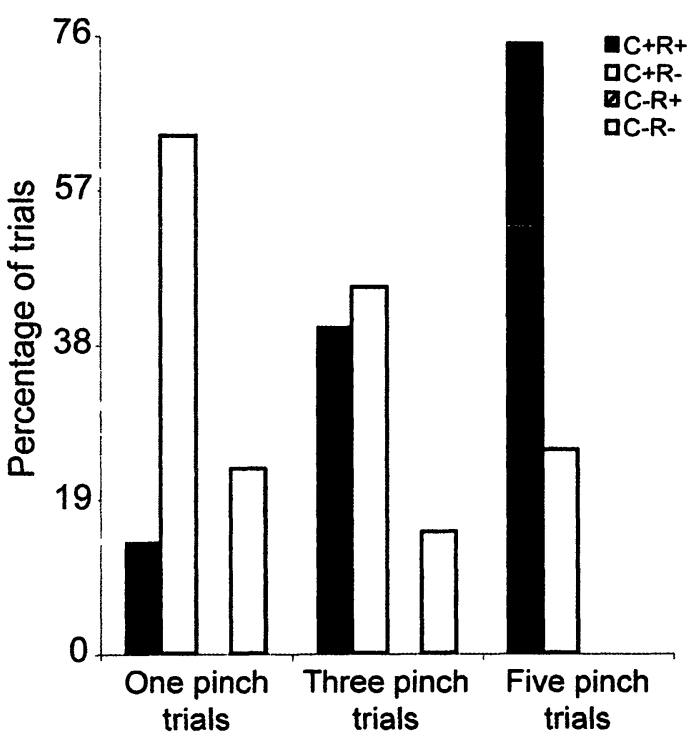

b

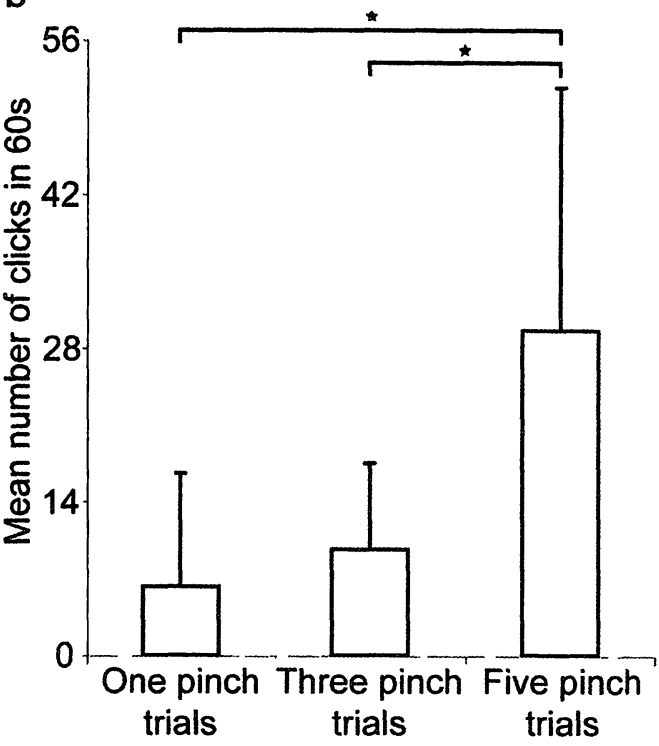

d

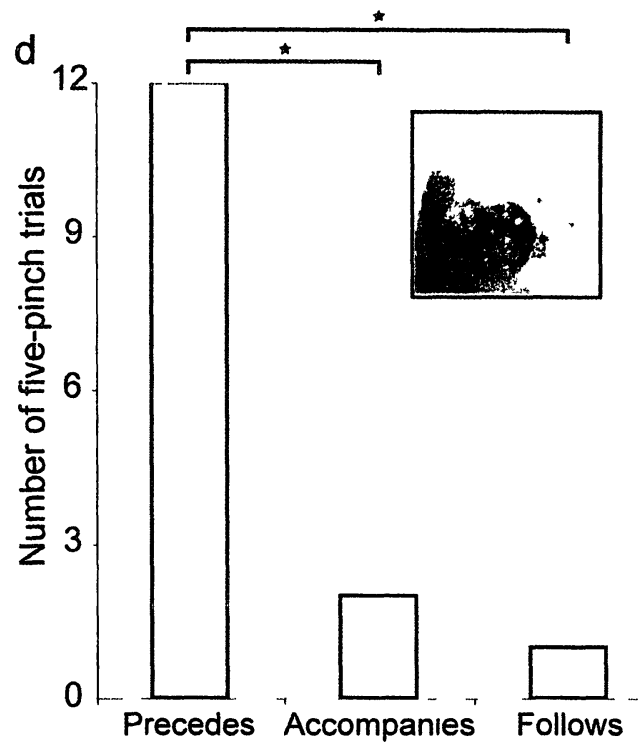

Figure 2.6 (a) Oscillograms of click trains following one-, three- and five-pinch trials. Arrows indicate when each larva was attacked. (b) Mean number of clicks produced during the first $60 \mathrm{~s}$ following the initial pinch in one-, three-, and five-pinch trials. (c) Behavioural responses to attack by forceps in one-, three- and five-pinch trials. $\mathrm{C}+\mathrm{R}+$, both clicking and regurgitation; $\mathrm{C}$ $\mathrm{R}-$, neither clicking nor regurgitation. Different letters indicates statistical significance. (d) The occurrence of clicking with respect to regurgitation (inset) in $\mathrm{C}+\mathrm{R}+$ five-pinch trials. Clicking preceded the appearance of regurgitation in most cases. 
$(n=20)$. Larvae pinched five times clicked significantly more $(29.60 \pm 22.09$ clicks $)$ than those pinched one $(6.23 \pm 10.34$ clicks $)$ or three times $(9.60 \pm 7.89$ clicks $)$ in a 60 second period (Fig. 2.6b, Mann-Whitney $\mathrm{U}, \mathrm{P}<0.001$ and $\mathrm{P}=0.001$, respectively).

Figure 2.6c shows the association between signalling and regurgitation for each attack type. Clicking without regurgitation $(\mathrm{C}+\mathrm{R}-)$ was the dominant response in one-pinch trials $(63.6 \%)$. However, this reaction decreased as the number of pinches increased to five (25\%). Conversely, the proportion of clicking with regurgitation $(\mathrm{C}+\mathrm{R}+)$ was positively correlated with the number of pinches. Few larvae in one-pinch trials had this reaction (13.6\%), whereas in five-pinch trials it was the most common response (75\%). In five-pinch trials where both clicking and regurgitation occurred, the first click preceded regurgitation significantly $\left(\chi^{2}=14.8, P=0.001 ;\right.$ Fig. 2.6d).

\subsection{Discussion}

In this chapter, I demonstrated that the tobacco hornworm, $M$. sexta, produces sounds that are clearly associated with being attacked, confirming observations by Brown et al. (2007) who first reported these sounds. Interestingly, there have been other papers on predator-prey interactions for M. sexta (e.g. Thurston and Prachuabmoh 1971, Stamp 2001, Thaler and Griffin 2008) and at least one specific study dedicated to the defensive responses of the caterpillar (Walters et al. 2001), yet there has been no mention of sound production. Possible explanations for this include that the caterpillars were stimulated in different ways and sound production was not elicited, though I varied the methods of attack in my experiments to match those of Walters et al., and sound was produced in 
each scenario. Another possibility is that sound production is a regional phenomenon found only in certain populations. I recorded from two sources in my experiments, one from eastern Quebec and the other from southern Ontario, and Brown recorded from a third from a population in south-central Nova Scotia. These locations are all from areas of eastern Canada, but are widely distributed within the region. Yet, since all individuals that were tested were from established laboratory colonies, they may, in fact, have all originated from a single source in North Carolina (Kingsolver 2007 and references therein). Experiments using wild-caught specimens and closely related species of the same genus should be carried out to determine if the sounds exist outside of these colonies. Another plausible explanation revolves around the characteristics of the sounds; though they are audible in a quiet room or at close distances, they are quiet in a noisy environment and, also predominately ultrasonic, so they may simply have gone unnoticed by other experimenters.

Individual clicks are produced using the mandibles where the inner ridges of one mandible catches on the anterior and outer ridges of the other as the mandibles are closed. The manner in which the different ridges catch onto each other may determine the number of components in each click. Mandible clicking has been reported in two other species of Bombycoidea caterpillars upon disturbance, Antheraea polyphemus and Actias luna (Brown et al. 2007), and is one of the limited options for sound production in a soft bodied insect such as a larva. Mandible clicking is not common in other insects, to my knowledge, but has also been reported in some grasshoppers during encounters with conspecifics and as a reaction to disturbance (Alexander 1960, Blondheim and Frankenberg 1983). Some suggestions have been made as to the mechanisms and 
functions of these sounds, though they have not been studied in detail. In Chapter 5 of this thesis additional species will be added to the list of mandible clickers and the relationship to mandible structure and possible evolutionary origins will be discussed.

As suggested by Brown, my data support the hypothesis that clicks produced by M. sexta caterpillars are defensive signals. Clicks were produced when the animal was attacked and the amount of clicks increased significantly as the degree of attack increased. Also, the natural predators of $M$. sexta should be capable of hearing the sounds; they are broadband with the majority of energy from $3-50 \mathrm{kHz}$ and temporally simple, thus resembling other insect disturbance signals permitting them to be perceived by a wide range of predators (Masters 1979, 1980). As with other species of sphingid caterpillars, $M$. sexta is presumably preyed upon by both vertebrate and invertebrate predators (Pittaway 1993, Tuttle 2007). Birds are a major predator of caterpillars and the sonic portions of the clicks overlap with the optimal frequency range of most avian predators (Schwartzkopff 1955, Dooling 1991). The dominant frequency of $M$. sexta clicks was ultrasonic, suggesting that they could be directed towards bats or mice. There is substantive evidence that caterpillars are common prey of gleaning bats, and that large caterpillars can form up to $30 \%$ of the diet of some species (Kalka and Kalko 2006, Wilson and Barclay 2006) and mice have been shown to respond to sounds produced by insects with similar sound frequencies (e.g. bee hissing, Kirchner and Röschard, 1999). Invertebrate predators like praying mantids are also capable of hearing ultrasound (Yager 1999) and could potentially be deterred by the signals as well.

Within the category of defence sound, my results support the hypothesis that these signals are aposematic. The main prediction for this hypothesis states that the sounds 
should be associated with an honest defence. My results indicate that clicking and regurgitation are strongly associated in this species, occurring together in $75 \%$ of all fivepinch trials. Furthermore, in the majority of these trials clicking preceded regurgitation significantly more than following it. In order to be an honest defence the regurgitant of M. sexta caterpillars should have some deterrent properties. Tobacco hornworn caterpillars feed on plants from the family Solanaceae that are known to contain tropane alkaloids which can act as lethal neurotoxins (Nishida 2002). Manduca sexta caterpillars are capable of storing the alkaloids, which, depending on the level of toxicity in the plant, can have lethal effects on predators (Rothschild et al. 1979).

Other behaviours were also observed in response to attack, including biting and thrashing, and would typically occur after the onset of sound production (when present). Walters et al. (2001) suggested that the thrashing behaviour exhibited by the caterpillars decreases the incidence of successful attacks on the prey by avian predators based on observed field encounters. This would increase the time spent in one place, a potentially dangerous activity for birds that are prey animals themselves (Kaby and Lind 2003). In addition, if the biting caterpillar came into contact with the delicate eye of a bird it could possibly be capable of inflicting serious damage. Therefore, the sounds may be a signal of general unprofitability, a signal to vertebrate predators that their time would be better spent elsewhere.

In summary, tobacco hornworm caterpillars produce defensive clicks using their mandibles in response to simulated attacks. The close association between the sounds and a potentially noxious regurgitation support the hypothesis that they function as an acoustic aposematic warning signal, though they may also act as a general signal of 
unprofitability. Future studies with live predators should be performed in order to determine which predators are capable of perceiving the signals, how they respond and if regurgitation, thrashing or biting is the effective defence. The close relationship between clicking and regurgitation or biting may give some clues to the evolutionary origins of this type of signaling. Since the mechanism involves the interactions of the two mandibles as they close, incidental noises produced as the two sides come into contact while performing other defensive behaviours, like regurgitating or biting, may have eventually led to the evolution of intentional signals to warn of the defence. These and other hypotheses are discussed in more detail in Chapter 5. 


\section{CHAPTER 3}

Acoustic stridulatory signals in caterpillars of the great peacock moth (Saturnia pyri: Bombyoidea, Saturniidae)*

*Parts of this chapter have been published.

Manuscript: Bura, V.L., Fleming, A.J., Yack, J.E. (2009) "What's the buzz? Ultrasonic and sonic warning signals in caterpillars of the great peacock moth (Saturnia pyri)" Naturwissenschaften, 96: 713-718

Statement of contributions: V. Bura collected and analysed the data, prepared the figures and wrote the paper. A. Fleming aided in the collection of data. 


\subsection{Introduction}

To date, experimental evidence exists for a single type of signalling mechanism mandible clicking - in caterpillars. Three species that have been reported to exhibit this behaviour, Antheraea polyphemus, Actias luna (Saturniidae, Saturniinae) (Brown et al. 2007), and Manduca sexta (Sphingidae, Sphinginae) (Brown et al. 2007, Chapter 2 of this thesis) all belong to the superfamily Bombycoidea, but to different subfamilies (Saturniinae and Sphinginae). This suggests that the mechanism is widespread or has evolved independently at least twice. In addition, based on a close temporal association, sounds in the three species have all been proposed to function as an aposematic warning of an impending regurgitant defence.

While surveying larval defensive behaviours in Bombycoidea and other outgroups (see Chapter 5) I observed that caterpillars of the Great Peacock moth (Saturnia pyri) produced long, repeated, high-pitched 'chirping' sounds that were distinct from the short clicking sounds reported in previous species. Also, unlike A. polyphemus, A. luna and M. sexta, S. pyri did not regurgitate. Previous studies on S. pyri show that they use phenolics and related compounds secreted from scoli (bristle bearing outgrowths) in combination with body thrashing as their defence strategy (Deml 2001, Deml and Dettner 1993, 1995). Interestingly, there was no reference to sound production in these studies, however I did find one anecdotal note that the caterpillars will "crunch their mandibles" when disturbed (Rougeot 1971). Since these insects represent a different form of sound production, possess different chemical defences and could be obtained in sufficient numbers to perform experimental trials, I decided to examine their sounds and defences in further detail. I hypothesize that sound production in S. pyri functions to warn predators of 
defensive chemical secretions. My goals were to characterize these novel sounds and their mode of production, and to test the hypothesis that they function in acoustic aposematism.

\subsection{Methods}

Animals

Saturnia pyri Schiffermüller eggs were obtained from wild caught females in Kloten, and Mohlin, Switzerland (Import Permits \#P-2007-03105 and \#P-2008-02614). Larvae were reared on cuttings of poplar (Populus sp.) housed in an insect rearing facility at Carleton University. All experiments were performed on late instars.

\section{Sound production mechanism}

Preliminary observations suggested that sounds were produced using the mandibles. This was investigated by examining the anatomy of the mandibles and with ablations. Larval mouthparts were videotaped during sound production using a Sony HDR-HC7 HD Handycam (Tokyo, Japan) equipped with a Sony ECM-MS957 microphone and a macro lens. Videos were analyzed using iMovie 3.0.3 on an Apple computer. During ablation experiments the anterior edges of both mandibles of known sound producers were ground down and the animal was subsequently tested for sound production. Larvae were anesthetised using carbon dioxide, and mandibles ground with a Vogue Professional 6700 nail drill (Woodland Hills, CA, USA) equipped with diamond dental burs. Controls were performed using a smooth bit that did not change the mandible structure. Mandible anatomy was examined by dissecting mandibles from known sound 
producers, sputter coating with gold-palladium and examining using a JEOL JSM-6400 scanning electron microscope (Tokyo, Japan).

\section{Sound recording and analysis}

Sounds analysed for spectral, intensity and temporal characteristics were recorded in an acoustic chamber (Eckel Industries Ltd., Cambridge, MA, USA), or in an enclosure lined with acoustic foam. Caterpillars were placed on a cutting of hostplant and induced to signal by delivering a pinch with forceps to the head capsule or posterior end. Sounds were recorded with a Brüel \& Kjær (Naerum, Denmark) 1/4" microphone type 4939 (grid off), placed approximately $5 \mathrm{~cm}$ away from the head capsule, amplified with a Brüel $\&$ Kjær Nexus conditioning amplifier type 2690, and recorded onto a Fostex FR-2 Field Memory Recorder (Gardena, CA, USA) at a sampling rate of $192 \mathrm{kHz}$. Analysis was performed using Raven Bioacoustics Research Program 1.2 (Cornell Laboratory of Ornithology, Ithaca, NY, USA).

Temporal characteristics, including train duration, number of chirps in a train, chirp duration and number of components in a chirp were measured from the first three trains of fourteen recordings. A train was defined as a series of chirps following an attack, until sound production ceased. A chirp was defined as the sound produced by a single movement of the mandibles, or (in other words) the smallest unit of sound distinguishable by the human ear (Broughton 1963).

Initial spectral analysis revealed that chirps differed in their frequency structure. Therefore I categorized chirps into multi- and single-component. Spectral characteristics were examined from five randomly chosen chirps for multi-component chirps, and all 
single component chirps from five animals. Spectra were produced using a 512-point Fast Fourier Transform (FFT) (Hann window).

Sound pressure levels were measured by recording caterpillar sounds at a distance of $10 \mathrm{~cm}$ using a Brüel \& Kjær 1/4" microphone and measuring peak to peak voltages on an oscilloscope (THS720A, Tektronix, Richardson, TX, U.S.A.). The voltages were converted to pascals based on the sensitivity output of the Nexus amplifier $(1 \mathrm{~V} / \mathrm{Pa})$ and subsequently transformed into dB SPL values (see Chapter 2 for additional details).

\section{Attack trials}

Attack trials were performed to examine the relationship between attack, sound production and other defences. Simulated attacks were performed by pinching the animal with blunt forceps, a technique commonly used to imitate an attack by a bird or the mandible bite of a predaceous insect (e.g. Bowers 2003, Grant 2006). Larvae were isolated on sprigs of poplar at least 30 minutes prior to experimentation. Five attacks were delivered to either the head-capsule or the posterior end of the larva, with approximately five seconds between attacks. In an additional trial set, one pinch was delivered to the posterior end of the caterpillar to assess any differences in response from the multiple-attack trials. Defensive behaviours were videotaped using a Sony DCRHC85 handycam equipped with a Sony Ecm-MS957 microphone placed 2-5 cm away from the head-capsule. Trials were analyzed using iMovie 3.0.3 to determine (i) the mean number of chirps in 60 s following the first attack, (ii) the occurrence of sound production and chemical release throughout the attack sequence, and (iii) the temporal relationship between defensive behaviours. 


\subsection{Results}

When disturbed, S. pyri caterpillars (Fig 3.1a) generated distinct, audible chirp trains and scoli secretions (Fig 3.1b). Caterpillars responded acoustically to a variety of disturbances, including touching the body, or agitating the plant sprigs, but the most reliable method to induce signaling was to grasp the larva's posterior end or head capsule, both of which induced different responses (see Attack section below).

\section{Sound production mechanism}

Video analysis and ablation experiments confirmed that sounds are produced by the mandibles. Each chirp is produced by either the right or left mandible (Fig. 3.1c) sliding against the inner surface of the opposite mandible, and each chirp train results from repeated movements of one particular mandible against the other. Once a chirp train is completed the larva may or may not switch sides between trains, but switching mandibles was never observed within a particular train. Both mandibles have serrated anterior edges (Fig. 3.1c-e), and I propose that the edge of one mandible scrapes against the inner surface of the other, where this is evidenced by distinct 'track marks' (Fig. 3.1d). These tracks occur on both mandibles, validating my observations that at least some individuals are 'ambidextrous'. The tracks are slightly textured (Fig. 3.1e), suggesting that the anterior mandible ridges rub against these inner 'teeth' to produce individual components, or 'toothstrikes', within a chirp. Grinding down the outer edges of either mandible so that contact between the two sides was not possible succeeded in eliminating sound production. All control animals continued to produce sound. 


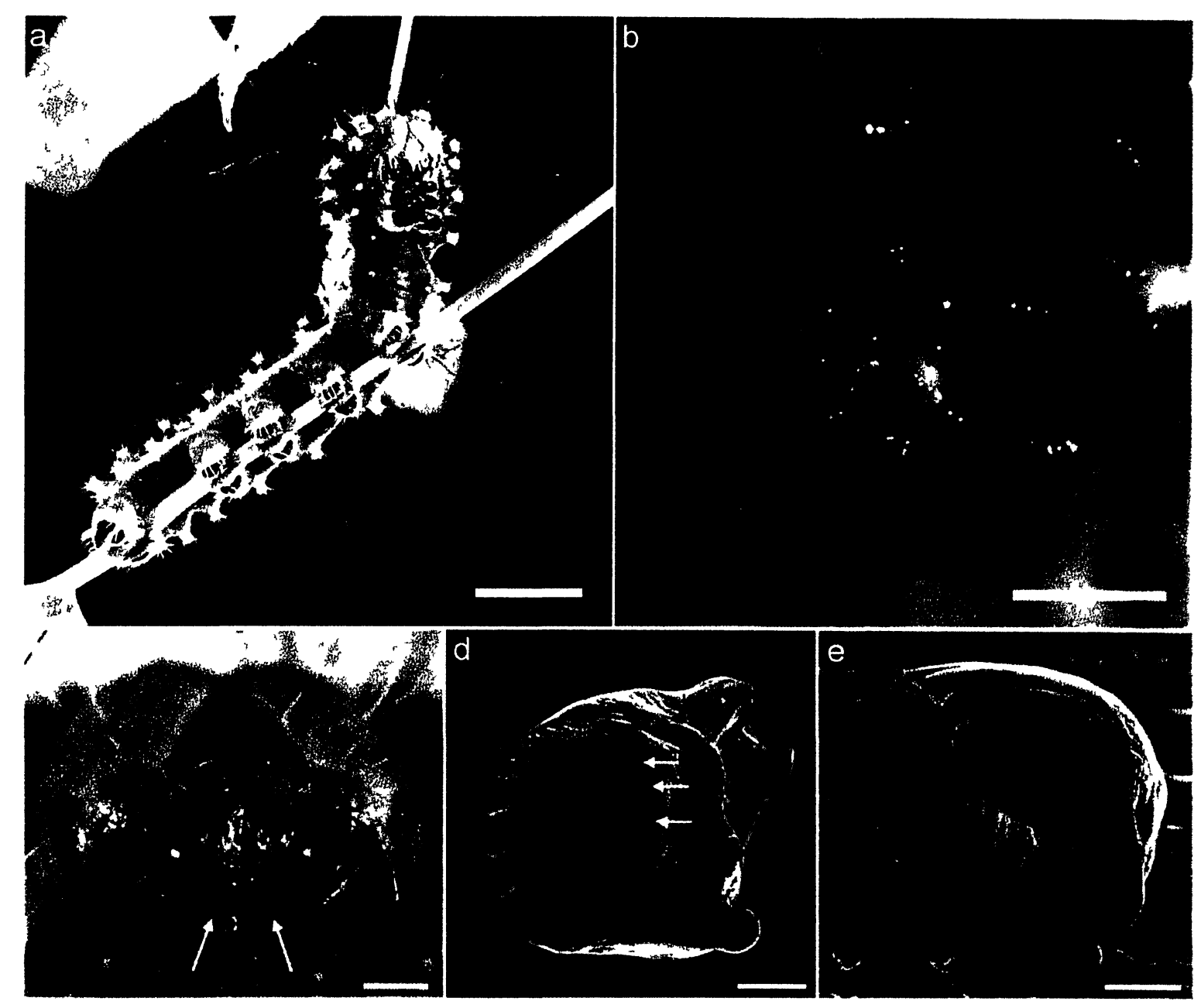

Figure 3.1 (a) A late instar Saturnia pyri larvae. Scale bar, $1 \mathrm{~cm}$. (b) Higher magnification of a scolus showing the bristle secretions. Scale bar, $1 \mathrm{~mm}$. (c) Light micrograph of the mouthparts with arrows showing the mandibles. Scale bar, $1 \mathrm{~mm}$. (d) Scanning electron micrograph of a right mandible with arrows indicating "track marks" from the opposite mandible. (e) Scanning electron micrograph of the left mandible showing the serrated outer edge. Scale bars, $0.5 \mathrm{~mm}$. 


\section{Sound characteristics}

Temporal patterns of sounds were analyzed from the first three trains of multiple attack trials (42 trains from 14 animals), where attacks were directed towards the posterior body region. Chirp trains ranged from 0.05 to 4.65 seconds (mean $1.70 \pm 1.12 \mathrm{~s}$ ) and contained on average $5.74 \pm 3.45$ chirps per train (Fig. 3.2a). Chirps occurring at the beginning of a train tended to be longer (mean duration $67.48 \pm 23.15 \mathrm{~ms}, \mathrm{n}=21$ ) and contained a greater number of components (mean $5.38 \pm 1.53$ ) than those that occurred at the end (mean duration $9.14 \pm 10.17 \mathrm{~ms}$, mean number of components $1.29 \pm 0.46$, $n=21)$.

Spectral analysis was performed on the first three chirp trains from five individuals where attacks were delivered to the posterior region. Sounds were broadband, with most energy between 3 and $90 \mathrm{kHz}$ (Fig. 3.2b,c). The energy distribution of multiand single-component chirps differed (Fig. 3.2c), with the former having more energy in the ultrasonic range (median dominant frequency $36.00 \mathrm{kHz}$, mean dominant frequency $34.45 \pm 11.93,88 \%>20 \mathrm{kHz}, \mathrm{n}=25$ ), and the latter having more energy in the sonic range (median dominant frequency $14.63 \mathrm{kHz}$, mean dominant frequency $21.57 \pm 15.19,70 \%$ $<20 \mathrm{kHz}, \mathrm{n}=23)$.

Sound levels were measured from 10 chirp trains obtained from four late instar larvae attacked from the posterior end. Maximum amplitudes, measured from the loudest component in a chirp, ranged from 74.2 to $79.3 \mathrm{~dB}$ SPL, measured at $10 \mathrm{~cm}$ from the source. 
a

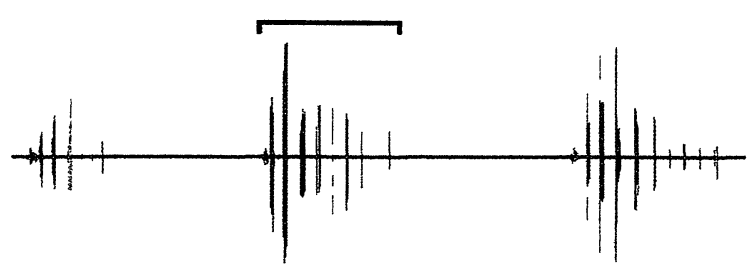

b
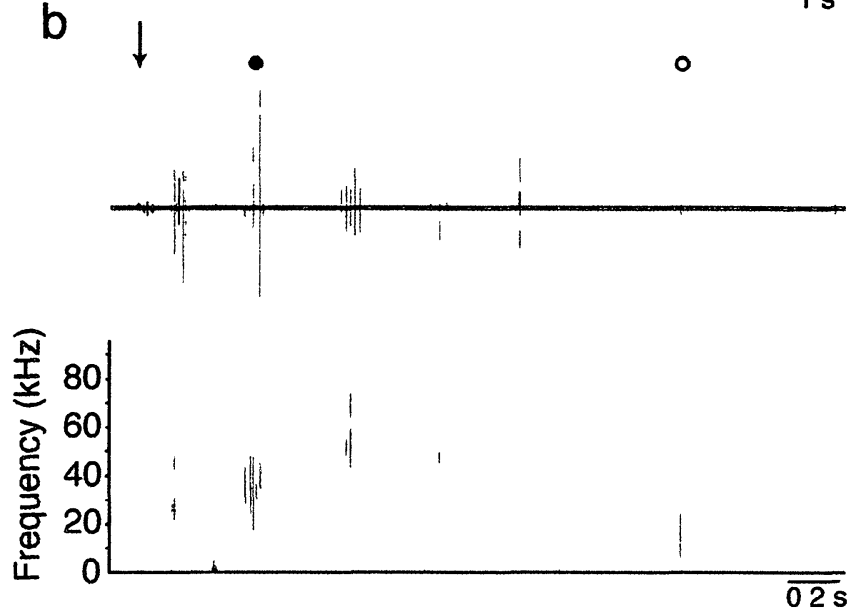

C Multi-component

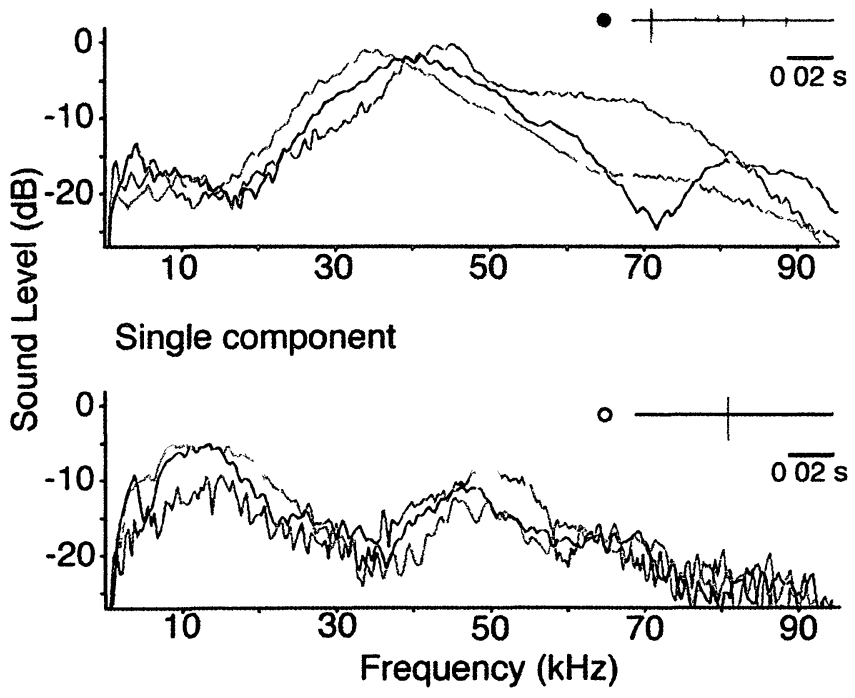

Figure 3.2 Oscillograms of Saturnia pyri sounds recorded from fifth instar larvae. (a) Three chirp trains following three consecutive attacks. (b) Time expansion of the bracketed train from part (a), showing typical multi-component (closed circle), and single component (open circle) chirps. The arrow indicates the time of attack. The accompanying spectrogram shows the frequency distribution of each chirp. (c) Power spectra of multi-component and single component chirps taken from three individuals. Time expanded chirps from (b) are inset for each type. 
Attack trials

A simulated predator attack induced a variety of defensive behaviours, including directed thrashing, head hiding/curling-in, secretion of chemicals from the scoli accompanied by a foul smelling odour, and sound production. Attacks to the head region typically resulted in the larva curling its head inward while producing sound and releasing chemical from the anterior scoli. In contrast, attacks to the posterior region caused the caterpillar to thrash towards the site of attack while signaling continuously and producing secretions from both the anterior and posterior scoli. Chirp rates following attacks to the posterior region $(24.95 \pm 19.04, \mathrm{n}=20)$ were significantly higher than those following attacks to the head $(12.20 \pm 16.79, n=20)(P=0.0107$ Wilcoxon Rank Sum test, $\left.\chi^{2}=6.5124\right)$ (Fig. 3.3a,b).

Overall, both acoustic signalling and chemical release increased with the degree of disturbance (Fig. 3.3a-c). During a 60 second period, larvae attacked five consecutive times signaled significantly more (24.95 \pm 19.04 chirps) than did those attacked once $(3.50 \pm 2.78$ chirps $)\left(P=0.0003\right.$ Wilcoxon Rank Sum test, $\left.\chi^{2}=13.3166\right)$. The relationship between sound production, chemical secretion and number of attacks is illustrated in Figure 3.3c. Following a single attack some caterpillars generated sound without secreting chemical $(\mathrm{S}+\mathrm{C}-)$, but when attacked multiple times, sound production was always accompanied by chemical secretion $(\mathrm{S}+\mathrm{C}+)$. In all five-pinch trials resulting in both sound production and chemical secretion, the first chirp preceded or accompanied the first droplet of chemical significantly more than following it $(\mathrm{P}=0.001, \chi 2=10.9, \mathrm{df}=1$; Fig 3.3d). 

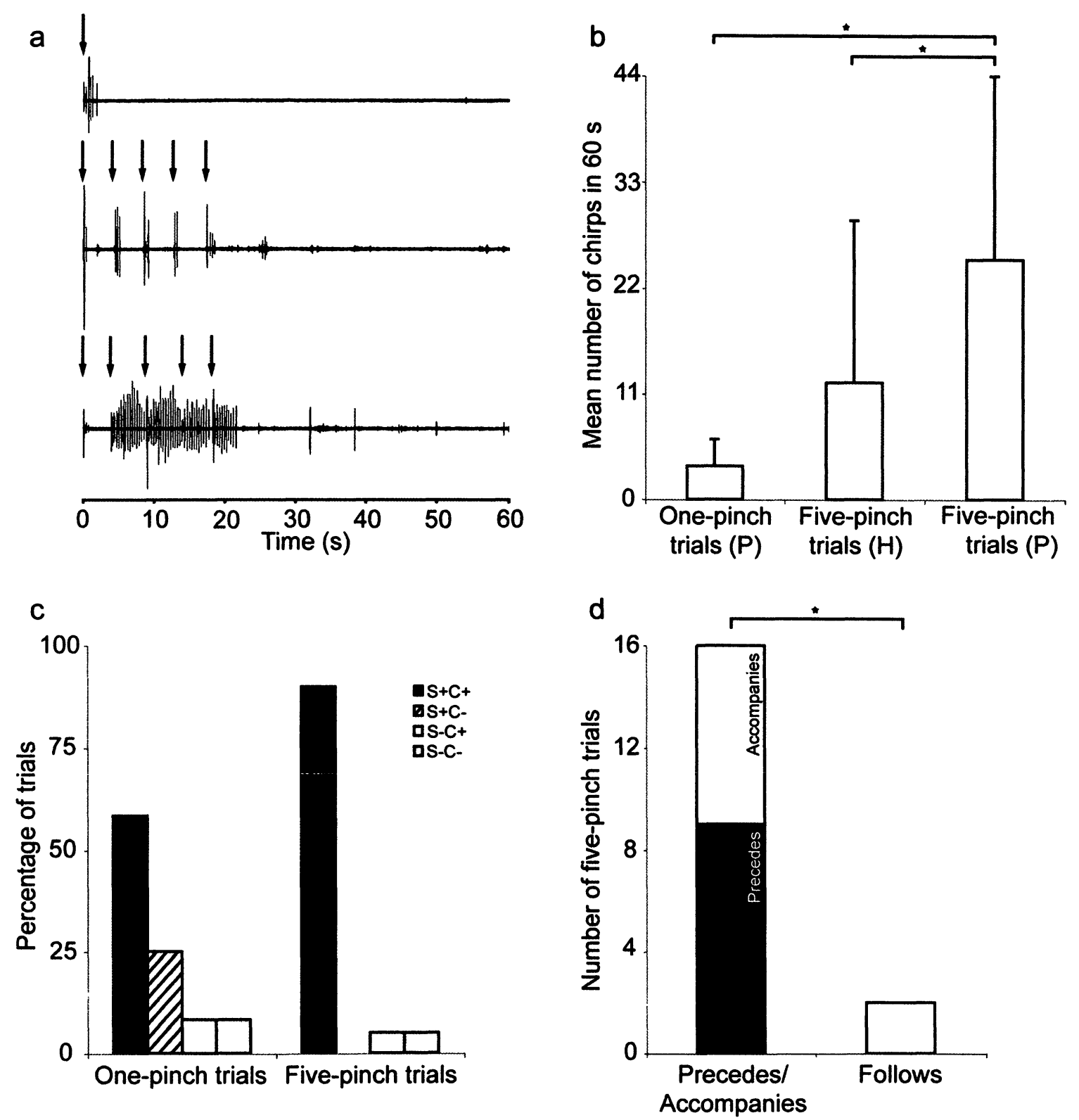

Figure 3.3 (a) Oscillograms showing typical responses to one- (posterior), five- (head), and five(posterior) pinch attack trials, with arrows indicating when larvae were attacked. (b) Mean number of chirps produced over 60 seconds following one-, five- (head), and five- (posterior) pinch attack trials. (c) Behavioural responses to increasing levels of disturbance (one and five pinch attacks, to the posterior region). $\mathrm{S}+\mathrm{C}+$, both sound and chemical production; $\mathrm{S}-\mathrm{C}-$, neither sound nor chemical production. (d) The temporal relationship between sound production and chemical release in five-pinch trials; the first chirp preceded or accompanied the secretion most often. 


\subsection{Discussion}

In this chapter I introduced a novel acoustic signal for caterpillars- mandibular stridulation. Stridulation is the production of sound by the movement of two body surfaces against each other, one possessing a stationary file (set of ridges), and the other a moveable scraper (a single ridge or knob) (Haskell 1961). Considered to be the most common method of sound production in insects (Haskell 1961, 1974), a well known example is found in crickets whereby one elytron overlaps the other and moves laterally to produce sound. I propose that individual chirps are generated when the serrated anterior edge of one mandible slides against the textured inner face, or 'teeth', of the opposite mandible to produce a series of components, or 'toothstrikes'. The variability in the inner surface of the mandibles, and the amount of pressure against the two parts may account for the diversity in the number of components per chirp. Unlike most examples of stridulation in arthropods, whereby the two body parts are specifically adapted for sound production (e.g. file and scraper) (Ewing 1989), the mandibles of $S$. pyri are not clearly differentiated. Stridulation using undefined areas has also been reported in other insects, for example undifferentiated elytral stridulation has been reported in a katydid and a locust for use in sexual behaviour (Dumortier 1963 and references therein). In fact, sounds produced by the mandibles in general are considered stridulation with nondifferentiated parts (Dumortier 1963), so this would also include mandibular clicking observed in other caterpillars.

My results support the hypothesis that sounds produced by $S$. pyri caterpillars are a defensive behaviour. Several predictions satisfy this hypothesis: First, sound production 
is strongly associated with a physical disturbance simulating a predator attack. Second, the number of acoustic signals generated is significantly correlated to an increase in the number of attacks. A third prediction is that natural predators of $S$. pyri should be capable of hearing these sounds. Saturnia pyri chirps resemble other insect disturbance sounds (Masters 1980) in that they are spectrally broadband and temporally simple, permitting them to be perceived by a wide range of predators. As with $M$. sexta caterpillars, birds, believed to be an important predator (Deml and Dettner 1993), bats, mice and mantids should all be capable of perceiving these sounds based on their frequency characteristics. Ants are also thought to be one of the main arthropod predators of $S$. pyri (Deml and Dettner 1995), and although ants do not possess ears (Yack 2004), they are sensitive to solid borne vibrations (Kirchner 1997). Although I did not test for vibratory stimuli directly, stridulations may be transmitted through the plant and function as a warning to ants. Indeed, wolf spiders are deterred by vibrational signals generated by stridulating insects (Masters 1979).

I hypothesize that stridulation in $S$. pyri is an aposematic display, warning predators of a chemical defence. To function as a warning signal, the sounds should be closely associated with an honest defence. My results demonstrate that stridulation most often precedes or accompanies scolus secretions, which in previous studies have been shown to contain compounds that function in deterring birds, ants, fungi, and microorganisms (Deml and Dettner 1993, 1995; Deml 2001). Interestingly, sound production is more pronounced during posterior attacks, corresponding to my observation that more chemical is secreted during these attacks. Overall, my results support the acoustic aposematism hypothesis. 
The evolutionary origins of chirping as a type of sound production may also stem from the interactions of the mandibles during other behaviours. The same chance production of incidental clicks during biting or regurgitation that I briefly discussed in the previous chapter may have also been responsible for stridulation, in this case limited to caterpillars whose mandibles lack ridges. Another possibility is that chirping evolved from clicking and represents a more ritualized signal. These hypotheses will be further discussed in Chapter 5. 


\section{CHAPTER 4}

Defensive whistles of the Walnut sphinx caterpillar, Amorpha juglandis (Bomycoidea: Sphingidae)*

*Parts of this chapter have been submitted to the Journal of Experimental Biology for publication (JEXBIO/2010/046805).

Manuscript: Bura, V.L., Rohwer, V.G., Martin, P.R., Yack, J.E. "Defensive whistling in caterpillars (Amorpha jugulandis, Bombycoidea): mechanisms and function"

Statement of contributions: V. Bura collected and analysed the data, prepared the figures and wrote the paper. V. Rohwer and P. Martin contributed the care, access to and knowledge of the avian predators. 


\subsection{Introduction}

In the previous chapters I have shown two different types of sound production in Bombycoidea caterpillars. Both clicking and stridulation are accomplished using the mandibles and both types of sound were closely associated with either regurgitation or chemical secretion. During the course of the survey (see Chapter 5) I encountered a caterpillar- the walnut sphinx- that produced a third type of sound that to my ear sounded like sqeaking or whistling, and did not appear to be associated with mandible movements. Also, I did not notice a clear association between sound production and a chemical defence, as seen in the previous examples. This species offered an opportunity to study another potential novel sound production mechanism in caterpillars, and explore a different possible defensive function. Since I was fortunate enough to rear multiple individuals, I chose to study this species in more detail.

In this chapter I introduce a novel form of sound production in caterpillars whistling. While most insects that generate sound do so by rubbing body parts together or against a substrate (Haskell 1961, 1974), few generate sound using air expulsion. Sounds produced by walnut sphinx (Amorpha juglandis) caterpillars have been described in passing as 'a note resembling the sound tcêp or tceep' (Sanborn, 1868), 'whistles', 'hisses', and 'squeaks' (Wagner 2005, Tuttle 2007), and due to the 'airy' nature of the sound, speculated to involve the spiracles (Wagner 2005). However, neither the sound characteristcs nor mechanisms have been formally studied. The purpose of this chapter will be to characterize the sounds produced by walnut sphinx caterpillars, test the 
hypothesis that they are produced by the spiracles, and gain insight into their defensive function by performing experiments with simulated and natural predator attacks.

\subsection{Methods}

Animals

Larvae of the walnut sphinx, Amorpha juglandis (JE Smith), were reared from eggs obtained from moths captured at ultraviolet lights at the Queen's University

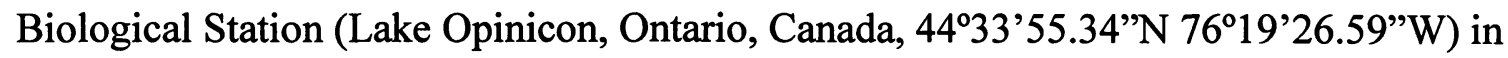
July 2008 and August 2009. Larvae were reared on cuttings of hop hornbeam (Ostyra sp.), alder (Alnus sp.), walnut (Juglans sp.) or beech (Fagus sp.). All experiments were performed on late $\left(4^{\text {th }}\right.$ or $\left.5^{\text {th }}\right)$ instar larvae. Caterpillars of Paonias myops (JE Smith), used for comparative purposes, were reared from eggs of moths captured at QUBS on cuttings of cherry (Prunus sp.).

\section{Sound recordings and analysis}

Sounds analyzed for spectral, intensity, and most temporal characteristics were recorded using a Brüel \& Kjær (Naerum, Denmark) 1/4" microphone type 4939 (grid on), amplified with a Brüel \& Kjær Nexus conditioning amplifier type 2690 , and recorded to a Fostex FR-2 Field Memory Recorder (Gardena, CA, USA) at a sampling rate of $192 \mathrm{kHz}$. Recordings were analyzed using RavenPro Bioacoustics Research Program 1.4 (Cornell Laboratory of Ornithology, Ithaca, NY, USA). Sound production was induced by placing an individual on a cutting of host plant and delivering an attack to the abdomen with blunt forceps (see below for details). All recordings were performed in an acoustic 
chamber (Eckel Industries Ltd., Cambridge, MA, USA).

Temporal characteristics including train duration, number of pulses in a train, inter-pulse interval and pulse duration were measured from the first three trains of ten individuals. A train was defined as a series of sound pulses following an attack, until sound production ceased. The first and third trains from ten individuals were compared using a paired t-test to determine if train duration differed depending on the sequence of the attack.

Initial spectral analyses showed that pulses differed in their frequency structure. Therefore, I categorized pulses into Types 1-3 (see results). Three pulses from each of five caterpillars were analyzed for Types 1 and 2, and a total of 14 pulses from five caterpillars were analyzed for Type 3 signals (due to a limited number of Type 3 signals in sound recordings used for frequency analysis). Measured characteristics included the dominant frequency, number of harmonics, and quality factors Q3 and Q10 (the dominant frequency divided by the frequency bandwidth at -3 and $-10 \mathrm{~dB}$, Ewing 1989). Power spectra were produced using a 512-point Fast Fourier Transform (Hann window, 50\% overlap). Further analyses of sound recordings obtained from videotaped trials (see below) from 32 animals were performed to determine the relative proportions of pulse Types 1-3, and the order that they occurred in a train.

Sound pressure levels were measured from 10 sound pulses from each of 5 caterpillars by recording with a Brüel \& Kjær 1/4" microphone type 4939 (grid on), and measuring voltages on a Tektronix THS720A oscilloscope. The voltages were converted to pascals based on the sensitivity output of the Nexus amplifier $(1 \mathrm{~V} / \mathrm{Pa})$ and subsequently transformed into dB SPL values (see Chapter 2 for additional details). 


\section{Sound production mechanism}

I tested the hypothesis that sounds result from the expulsion of air through spiracles, and further identified the specific source of sound production using high-speed videography, selected reversible occlusions, and laser vibrometry.

Experiments using high speed video were performed to examine body movements associated with sound production. A larva was placed on a wooden stick and induced to signal. A Lightning RDT (High Speed Imaging Inc., Markham, ON) camera captured 500 frames per second using Xcitec MiDAS 2.0 software (Cambridge, MA) on an A70 Toshiba Satellite (Tokyo, Japan) notebook computer. Sounds were recorded simultaneously using a Brüel \& Kjær 1/4" microphone (see above) and a MiDAS DA123 Data Acquisition Module (Cambridge, MA). The relationship between body movements and sound production was analyzed using Xcitec MiDAS 2.0 software.

Reversible spiracular occlusions were performed to determine if spiracles were involved in sound production, and if so, which ones. Caterpillars previously determined to produce sounds were anesthetized using carbon dioxide, and clear make-up latex (LR1, Ben Nye Company Inc., Los Angeles, CA) was applied to all abdominal spiracles using the provided application brush. The latex would turn from white to clear when it had dried which typically took less than 3 minutes. The larva was placed on its dorsal side once the latex had dried, and tested for sound production 5 minutes after it had righted itself to ensure that all caterpillars tested were at the same level of alertness. To elicit sound production a pinch was delivered to the abdomen, and the sound (or lack thereof) was recorded using a Sony ECM-MS908C microphone and Sony DCR-TRV19 camera (Tokyo, Japan). The latex was then gently removed and the caterpillar was re- 
tested for sound production. Selected occlusions were then performed on specific pairs of spiracles to determine which were involved in sound production.

Laser Doppler Vibrometry was used to confirm that sounds were being produced by selected spiracles. A caterpillar was placed on a wooden stick so that the spiracle of interest was positioned perpendicular to a thin strip of lens paper (Ross Optical, Ladd Research Industries Inc., Burlington, VT) suspended across a wooden frame. The beam of a laser vibrometer (PDV-100 Polytec Inc., Irvine, CA, USA) was reflected off the tissue paper using a reflective disc. Signaling was induced and vibrations created by the sounds (or lack thereof) detected by the laser (Velocity $22 \mathrm{~mm} / \mathrm{s}$; high pass filter off; Low Pass Filter $20 \mathrm{kHz}$ ) were recorded to a Marantz data recorder (PMD 671, D\&M Professional, Itasca, IL) at a sampling rate of $44.1 \mathrm{kHz}$.

\section{Anatomy of spiracles}

External anatomy of the abdominal spiracles of $A$. juglandis was examined to assess size differences between sound- and non-sound producing spiracles. Spiracles of 5 preserved caterpillars were photographed using an Olympus SZX12 (Olympus Corporation, Tokyo, Japan) light microscope equipped with an AxioCam MRc5 (Carl Zeiss Micro Imaging $\mathrm{GmbH}$, Göttingen, Germany) camera. Images were captured using AxioVision 4.6 and the length and width of spiracles were measured using ImageJ 1.42q (National Institutes of Health, USA). Mean sizes of the sound producing spiracle were compared to all others using an ANOVA and post hoc analyses were conducted with a Tukey - Kramer HSD using JMP8 statistical software. For comparison, spiracles in $P$. myops, a similarly sized but silent species from the same subfamily as $A$. juglandis were examined as outlined above. 


\section{Attack trials}

Attack trials were performed to examine the relationship between attack, sound production and other defences, and to assess the response of a predator to the sounds. Simulated attacks using forceps and natural attacks with an avian predator were conducted. All experiments were carried out on caterpillars resting on a sprig of hostplant, and videotaped using a Sony DCR-HC85 Handycam equipped with a Sony ECMMS957 microphone. Analysis of the trials was performed using iMovie 7.1.4.

During simulated attack trials larvae were isolated on sprigs at least 30 minutes prior to experimentation. Attacks with blunt forceps were conducted to simulate an attack by a bird or the bite of a predaceous insect (e.g. Bowers, 2003; Grant, 2006). Ten trials were performed where the resting caterpillar was attacked once near the head or abdomen and then quickly released. To further assess how an animal responded to multiple attacks, 8 trials were performed where attacks were repeated 5 times with approximately 5 seconds between successive attacks. Trials were analyzed to determine the number of caterpillars that responded with sound on the first or subsequent attacks, the number of pulses produced over a $60 \mathrm{~s}$ period, and to document other defensive behaviours associated with sound production.

Predator trials were conducted with three captive yellow warblers (Parulidae, Dendroica petechia) at Queen's University in Kingston, Ontario, Canada. Yellow warblers are a likely predator of the walnut sphinx, since this bird frequently eats caterpillars (Lowther et al. 1999), and overlaps in distribution with the walnut sphinx (American Ornithologists' Union 1998, Tuttle 2007). All yellow warblers were housed in individual cages $(45.7 \times 45.7 \times 91.4 \mathrm{~cm})$ with access to food (Exact Rainbow 
Canary/Finch diet, Kaytee Products Inc., Chilton, Wisconsin, USA) and water ad libitum. The birds were accustomed to searching foliage in their cage for prey items and had been previously fed live caterpillars of various species. Birds were born in the wild, but were raised in captivity from about 7 days old. For each trial, a caterpillar was placed on a twig inside the bird's enclosure amidst the other vegetation and close to at least one perch. Trials began when the experimenter had moved away from the cage $(\sim 2 \mathrm{~m})$ and ended when the bird no longer showed an interest in the caterpillar. One trial was conducted for each bird and trials lasted between $6-16$ minutes. This work was approved by the Queen's University Animal Care and Use Committee (protocols \# 100340, 100168) on birds that were held in captivity under Canadian Wildlife Service permit CA0243. Trials were analyzed for the caterpillar's defensive behaviour (including sound production), the number of times the birds attacked, the latency to the first attack from the beginning of the trial, and the birds' reactions to the sounds.

\subsection{Results}

Walnut sphinx caterpillars consistently generated audible 'whistles' when attacked and sounds were sometimes accompanied by other defensive behaviours (see below). There was no evidence of caterpillars responding acoustically to the presence of conspecifics, and sound production was not detected in early instars (1-3).

\section{Sound characteristics}

Temporal characteristics were measured from the first three trains from 10 animals $(n=30)$. Train durations ranged from 90 to $8764 \mathrm{~ms}($ mean $2626 \pm 2339 \mathrm{~ms})$ and 
contained $1-8$ pulses (mean $3.47 \pm 2.43$ ) (Fig 4.1a). Within a given trial, the durations of the first and third trains were compared, and no significant differences were observed (paired t-test, two-tailed, $\mathrm{P}=0.88$ ). Pulse durations ranged between 44 and $2060 \mathrm{~ms}$ (mean $440 \pm 272 \mathrm{~ms})(\mathrm{n}=101)$, with Type 2 pulses being longer than Types 1 and 3 (Table 4.1), and the mean inter-pulse interval was $501 \pm 282 \mathrm{~ms}(\mathrm{n}=72)$.

Spectral analysis revealed 3 general categories of pulses (Types 1-3) based on their frequency structure (Fig 4.1 b,c; Table 4.1). Type 1 pulses are broadband with a distinct multi-harmonic series occurring near the beginning of the pulse. The harmonic portion had a mean peak frequency of $22 \mathrm{kHz}$ occurring at harmonic $6 \pm 3$, and the broadband portion had a peak frequency at $23 \mathrm{kHz}$ (Fig 4.1a; Table 1). Type 2 pulses were broadband throughout with a mean peak frequency of $15 \mathrm{kHz}$. Type 3 pulses, or 'pure whistles', were multi-harmonic (mean $3 \pm 1$ ) with a mean peak frequency at 10 $\mathrm{kHz}$, occurring at harmonics 1 or 2 . Type 2 pulses were the most common, followed by Type 1 and then Type 3. In an analysis of attack trials in 32 animals, Types 1, 2 and 3 occurred at least once in $81.25,100$, and $43.75 \%$ of the trials respectively. There was also a trend with respect to where each type occurred during a train, with $100 \%$ of Type $1 \mathrm{~s}$ occurring in the first third of a train, while $42.6 \%$ and $68.9 \%$ of Types 2 and 3 occurred in the middle and last third of the train respectively.

Sound pressure levels ranged from 82.1 to $86.9 \mathrm{~dB}$ SPL at $10 \mathrm{~cm}$. The relative intensity of pulses in a typical train declined from the beginning to the end, with the first being 4.15 times greater in amplitude than the last $(n=12$ trains from 4 animals). 
a

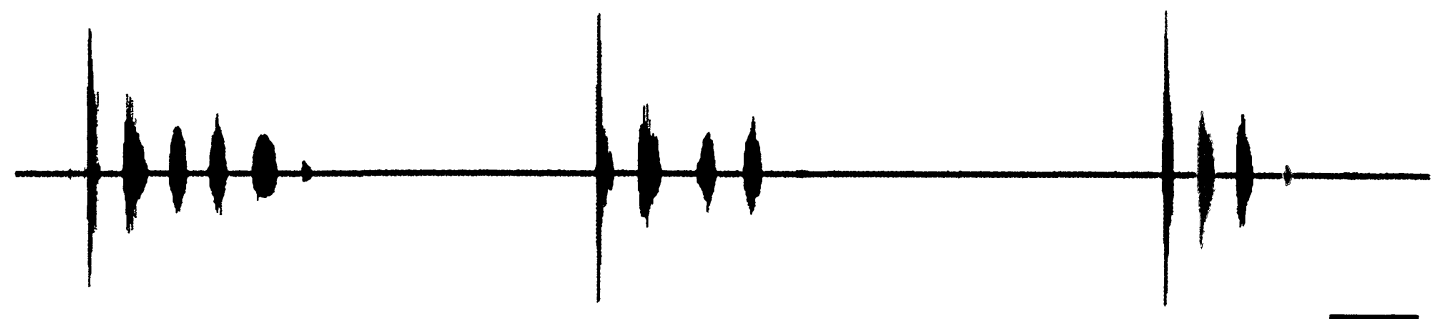

b
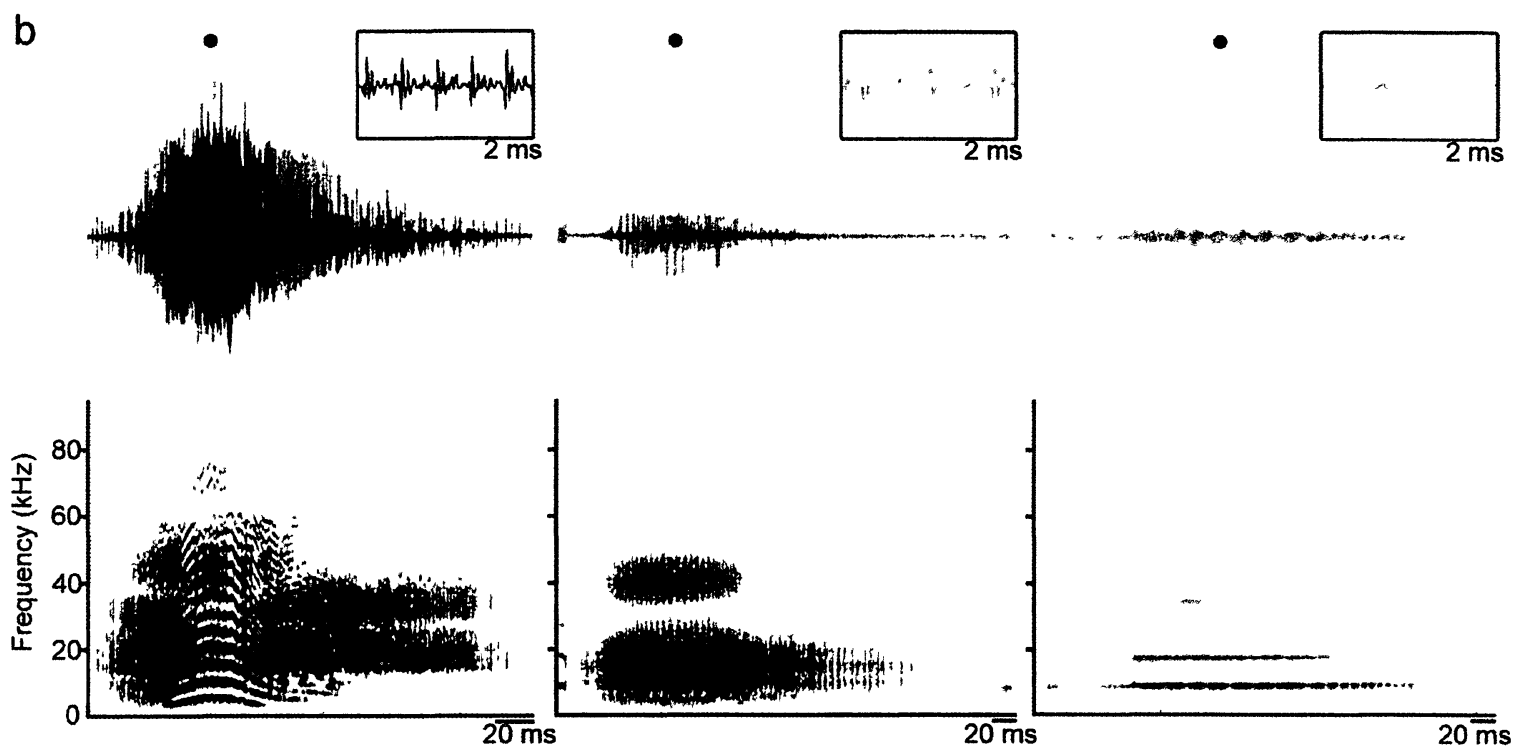

C

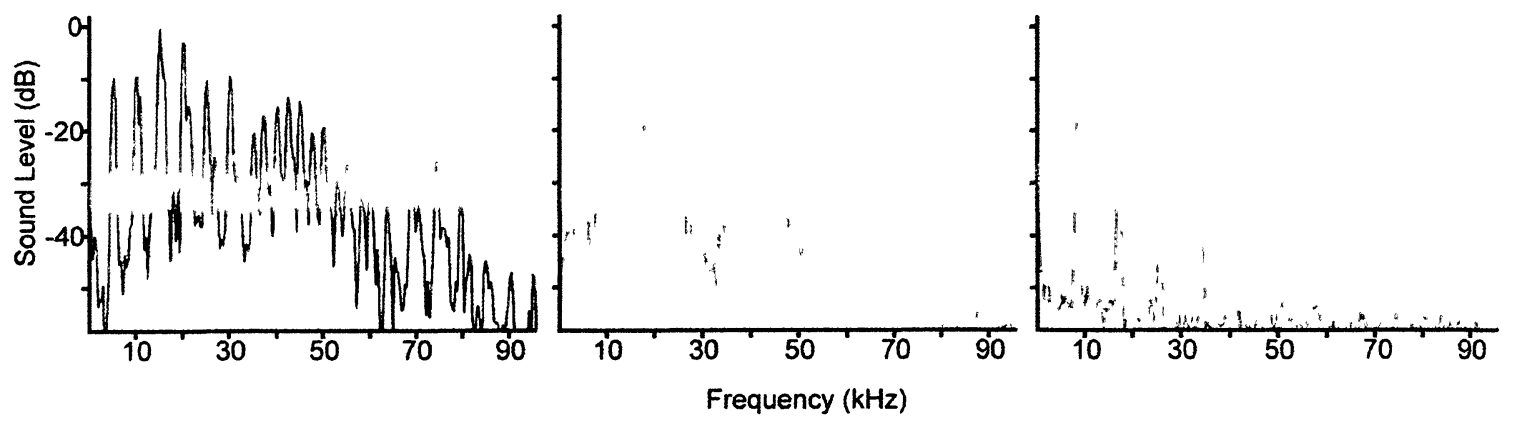

Figure 4.1 Amorpha juglandis sounds recorded from fifth instar larvae. (a) Oscillogram of three pulse trains following three consecutive attacks. Attack onset is marked with an arrow. (b) Oscillograms and corresponding spectrograms of time expanded pulses from the last train in (a), showing from left to right, Types 1,2 and 3. Insets represent an expanded time scale of the area represented by the black circle. (c) Power spectra of the 3 pulse types taken from the area represented by the black circle in (b). 
Table 4.1 Spectral and temporal characteristics of pulse types (mean \pm s.d.).

\begin{tabular}{|l|c|c|c|c|}
\cline { 2 - 5 } \multicolumn{1}{c|}{} & \multicolumn{2}{c|}{ Type 1 } & Type 2 & Type 3 \\
\cline { 2 - 5 } \multicolumn{1}{c|}{} & Narrowband & Broadband & & \\
\hline Peak frequency $(\mathrm{kHz})$ & $22.18 \pm 8.49$ & $23.17 \pm 7.99$ & $14.80 \pm 2.31$ & $9.54 \pm 3.65$ \\
\hline Q3 & $40.67 \pm 42.38$ & $15.65 \pm 9.26$ & $7.39 \pm 5.22$ & $19.00 \pm 5.67$ \\
\hline Q10 & $13.81 \pm 7.86$ & $1.48 \pm 0.55$ & $1.02 \pm 0.27$ & $9.90 \pm 3.79$ \\
\hline \# of harmonics & $8-34$ & - & - & $2-5$ \\
\hline Pulse duration (ms) & $250.67 \pm 104.92$ & $250.67 \pm 104.92$ & $423.27 \pm 191.43$ & $240.29 \pm 82.24$ \\
\hline \# of pulses (n) & 15 & 15 & 15 & 14 \\
\hline \# of animals (N) & 5 & 5 & 5 & 5 \\
\hline
\end{tabular}




\section{Sound production mechanism}

Initial investigations into the mechanism of sound production involved videotaping body movements that accompanied sound production, and obstructing all spiracles. Sound production was always accompanied by contraction of the anterior body segments (thorax and first 2 abdominal segments) (Fig 4.2). On average, the contraction lasted $403.5 \pm 192.7 \mathrm{~ms}$ and began $97.5 \pm 111.1 \mathrm{~ms}$ before the onset of signaling $(\mathrm{n}=5)$. Following the contraction, it took $156.0 \pm 35.6 \mathrm{~ms}$ to return to full extension. When all abdominal spiracles were obstructed $(n=5)$, sound production was eliminated, but when the spiracles were uncovered, sound production resumed. Selective obstructions and laser vibrometry experiments were then performed to determine which spiracles were involved. Selected ablations began by applying latex to all of the spiracles, and then removing the latex from the anterior most spiracles. Sound production did not occur until the $8^{\text {th }}$ abdominal spiracles (A8) were uncovered. We next applied latex to only the A8 spiracles, and sound production was eliminated in $100 \%$ of trials $(n=10)$. Once the latex was removed, sound production returned in $100 \%$ of trials. The ability to signal was never lost in animals where other spiracles had been blocked $(n=5)$. Laser recordings $(n$ $=5$ ) confirmed that sound is produced by the movement of air through A8 (Fig 4.3). There was evidence of a small amount of vibration when recording over other spiracles (Fig 4.3), but this occurred regardless of whether that spiracle was exposed or covered. Therefore, these vibrations were being picked up indirectly from sounds produced by A8.

\section{Spiracle anatomy}

External measurements of the spiracles in A. jugulandis revealed that A8 was longer than the other 7 abdominal spiracles (A8 and A7, P =0.0007; A8 and all other 


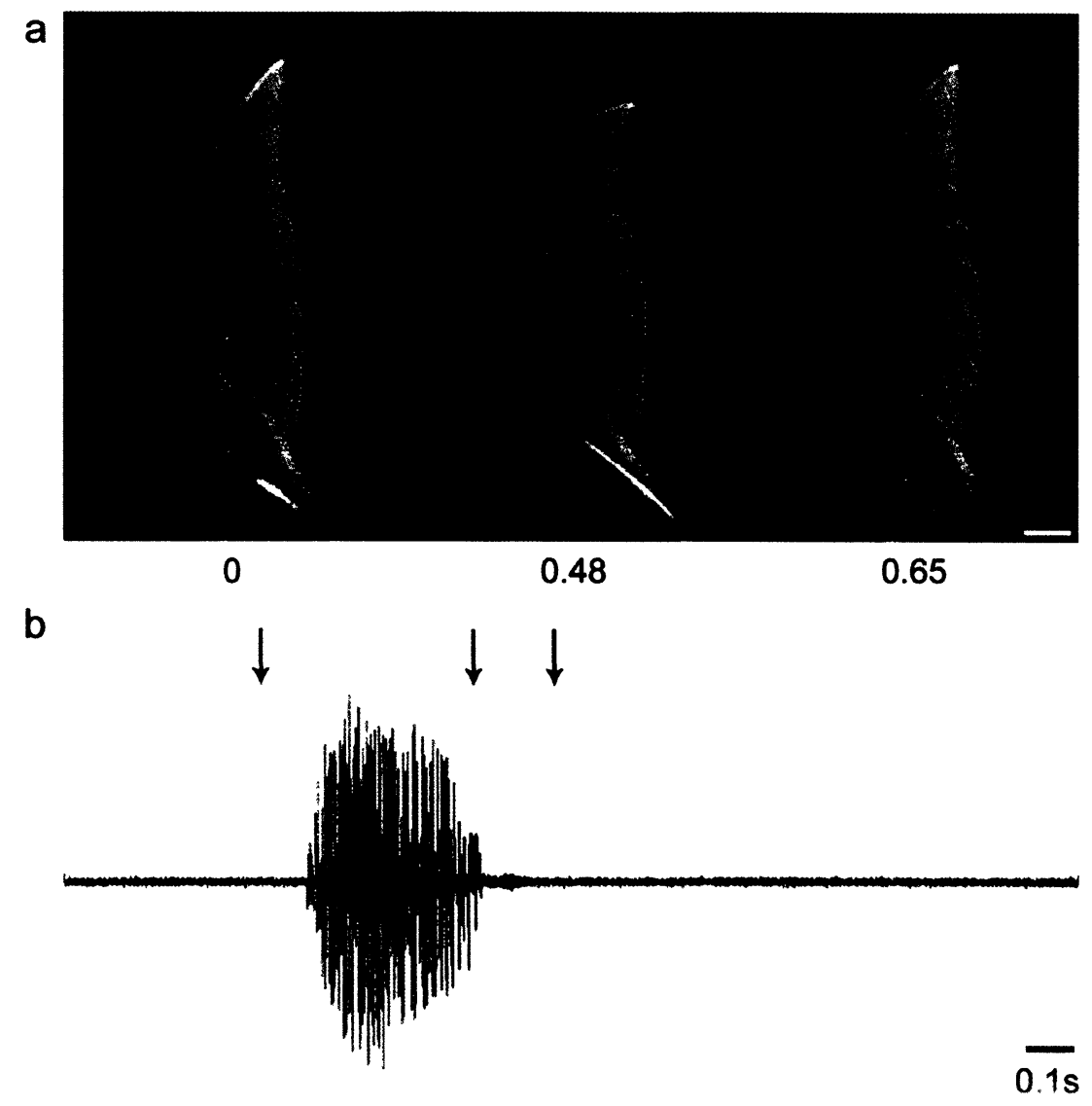

Figure 4.2 Body movements associated with sound production. (a) High speed video frames of a larva during sound production showing from left to right, the beginning of compression, full compression, and full extension (scale bar, $2.5 \mathrm{~mm}$ ). Times of occurrence for each frame are indicated below each frame. (b) Oscillogram of the associated sound with arrows marking the occurrence of the frames in (a). 


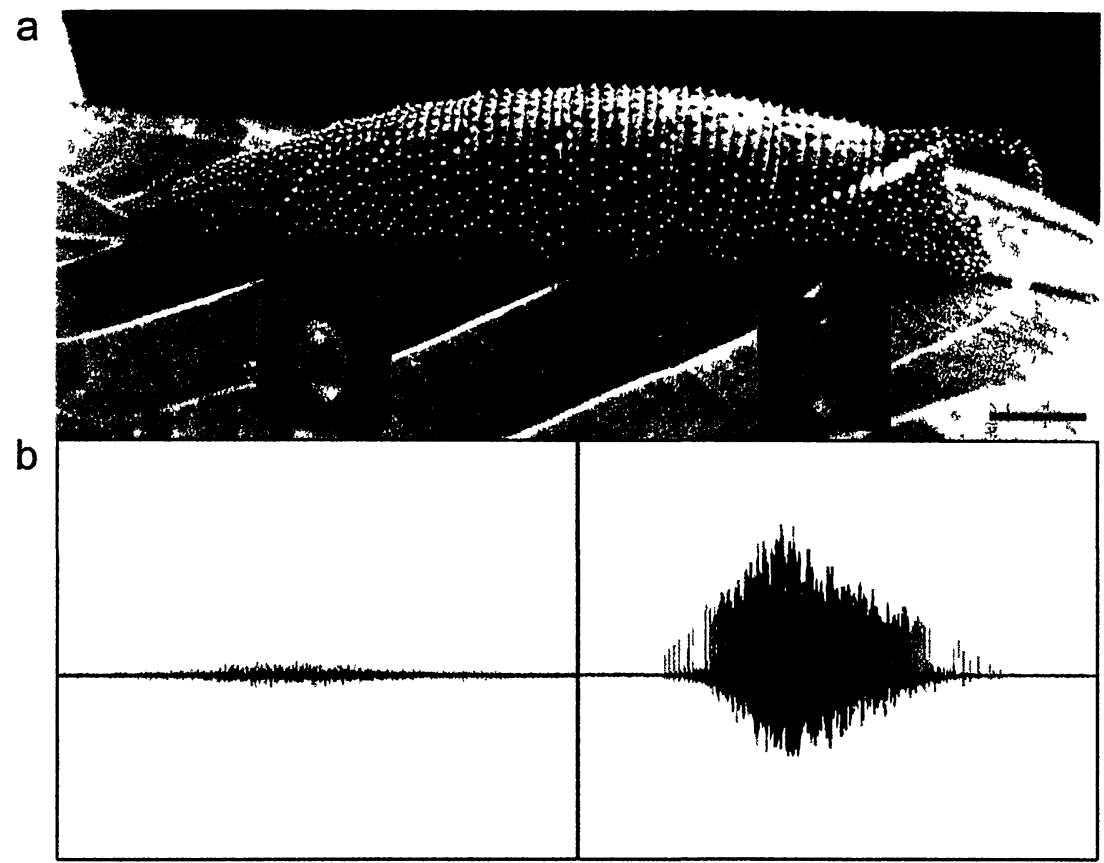

Figure 4.3 Spiracle anatomy and air expulsion. (a) A fifth instar caterpillar (scale bar, $5 \mathrm{~mm}$ ) showing $5^{\text {th }}$ and $8^{\text {th }}$ abdominal spiracles magnified in the inset (scale bars, $0.25 \mathrm{~mm}$ ). (b) Oscillograms obtained from laser recordings showing the movement of air produced by a sound pulse when either positioned above the $5^{\text {th }}$ (left) or $8^{\text {th }}$ (right) spiracles. 
spiracles $\mathrm{P}<0.0001$ ) (Fig 4.3a), and wider than one spiracle (A8 and $\mathrm{A} 1, \mathrm{P}=0.001)$. This pattern was not repeated in $P$. myops, where there was only a significant difference between the length and width of spiracles A8 and A1 $(P=0.0007$ and 0.0056 , respectively).

\section{Attack experiments}

Simulated attacks consisted of either one or five pinches being delivered with blunt forceps. In one-pinch attacks sound production occurred in 9 out of 10 trials, with an average of $5.20 \pm 3.97$ pulses per 60 second interval. Sound production was elicited in all five pinch trials $(n=8)$ with an average of $9.25 \pm 7.78$ sound pulses per 60 second interval. When attacks were directed to the posterior abdomen the caterpillar would release its first whistle while thrashing once towards the site of attack, presumably intending to bite the attacker. The caterpillar would then return to its resting position and continue its sound pulse train coupled with the telescoping body movements. Anterior attacks elicited the same series of behaviours except that the caterpillar would flick its anterior body away from the pinch instead of towards it. Regurgitation was observed only once during a one pinch trial, and was not observed in any of the 5 pinch trials.

Trials using yellow warblers were performed to assess the behaviours of both the caterpillar and an avian predator. An attack event was defined as the bird pecking at the caterpillar one or more times in a row following an inspection. Each of the three birds attacked the caterpillar more than once $(2.67 \pm 0.58$ times; $n=3)$, and each attack provoked the caterpillar to make sounds. Acoustic responses were similar to those in the simulated attacks, with train durations of $2800 \pm 2250 \mathrm{~ms}$, and $1-8$ pulses per train $(\mathrm{n}=$ 13). The larva never produced sound prior to the initial attack in a trial. However, once 
each bird provoked the caterpillar to produce sound, the caterpillar signalled when a movement by the bird caused the leaf the caterpillar was resting on to move. Sound production by the caterpillar occurred immediately when the bird's beak made contact with its body. Thrashing responses by the caterpillar were similar to those observed in forceps attacks, whereby attacks to the anterior caused the caterpillar to flick its head away from the bird, and posterior attacks caused the caterpillar to arc its head dorsally towards the site of attack. As far as I could determine, the caterpillar's head never made contact with the bird, and regurgitation was not observed in these trials.

Following the onset of a trial, the latency to the birds' initial attacks was on average $140.00 \pm 112.07 \mathrm{~s}$, increasing to $223.33 \pm 98.65 \mathrm{~s}$ for the second attack and decreasing to $193.00 \pm 32.53 \mathrm{~s}$ for the third. The birds responded to the first train of sounds by flinching, cocking their heads and inspecting, and eventually moving away from the caterpillar by hopping or flying away. Each bird attempted a second attack and responded to the caterpillar's sounds by again moving away. In one case, the response was dramatic, with the bird diving down and away from the caterpillar into thicker vegetation as if attacked by a predator (Fig 4.4, Gaddis 1980, Lima 1993). Two of the three birds returned for a third attack, and following sound production by the caterpillar, did not return again. The caterpillar remained unmolested in the cage with the bird for an average of $8 \mathrm{~min} 59 \mathrm{sec} \pm 5 \mathrm{~min} 49 \mathrm{sec}$ after the last attack, after which the trials were terminated. Caterpillars involved in our trials survived the attacks by yellow warblers and exhibited no obvious wounds from the attacks.

In summary, during all of the attack trials, sound production was the most consistent response to attack. Upon the first attack during a trial, sound production was 


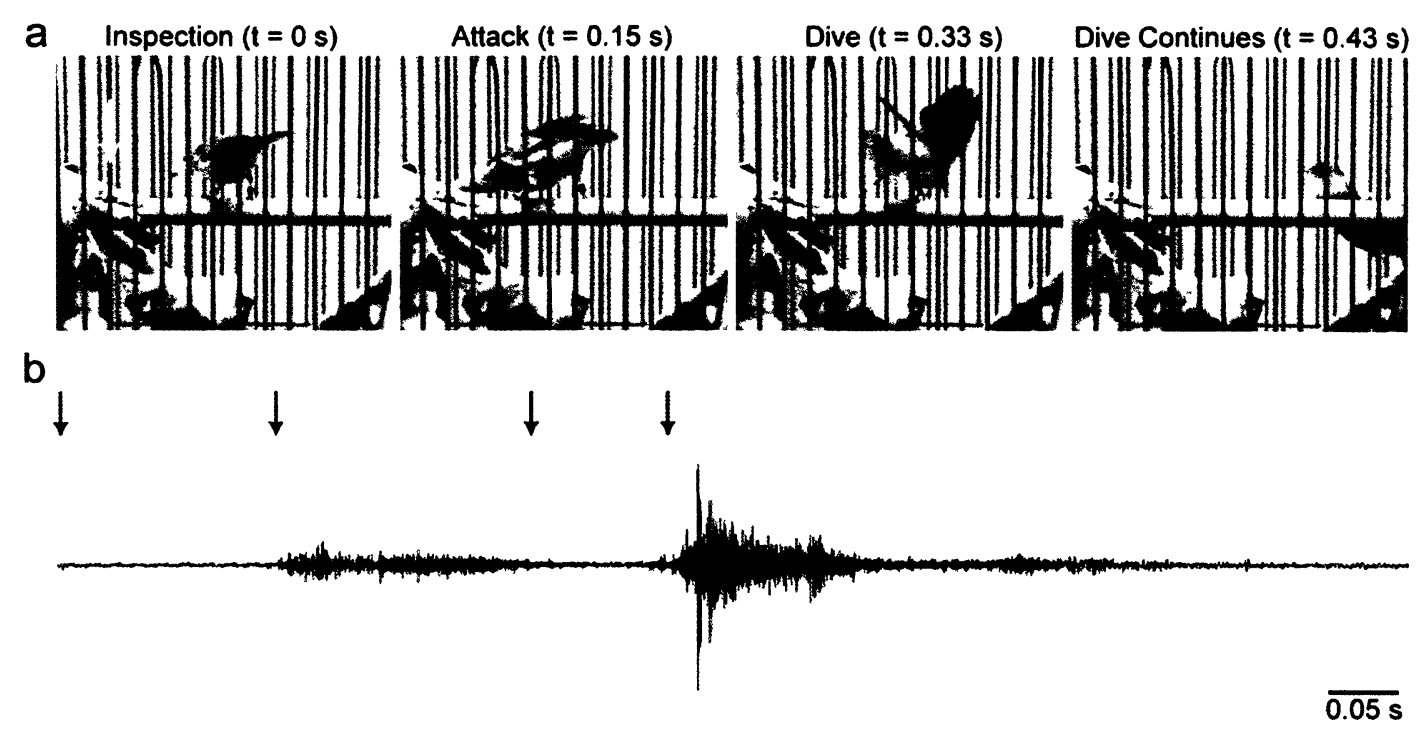

Figure 4.4 Reaction of a yellow warbler to the defensive sounds of the walnut sphinx caterpillar. (a) Video frames of the encounter with the time of occurrence in brackets. The white arrow in frame 1 indicates the position of the caterpillar. (b) Oscillogram of the caterpillar sounds with arrows corresponding to the onset of each video frame shown in (a). The sound pulse following the $4^{\text {th }}$ arrow is the noise produced by the bird's retreat. 
accompanied by directed thrashing, but upon subsequent attacks, sound production was not necessarily accompanied by thrashing. Regurgitation was rare during attack trials. However, I noted that outside of formal attack trials, regurgitation was observed in situations when it was necessary to handle the caterpillars for extended periods, such as when they were being set up for 'ablation' trials, or when changing the food in their enclosures. During these times caterpillars would regurgitate more readily, and try to bite, but such behaviours were typically not accompanied by sound production. During these periods the caterpillars were not squeezed or handled roughly and although not formally tested, I argue that these defensive responses resulted from continual handling and being restrained.

\subsection{Discussion}

In this chapter I have identified and described a novel form of sound production for caterpillars - whistling - and performed preliminary trials on the function of these sounds. The following discussion focuses on the mechanism of sound production through air expulsion in insects, and reviews the results with respect to the function and evolutionary origins of this rare form of signaling in caterpillars and other insects.

\section{Sound production mechanism}

My results support the hypothesis that the sounds of walnut sphinx caterpillars are produced by air expulsion. Reports on this mode of sound production in insects are extremely rare (Haskell 1974, Ewing 1989). The Death's Head hawkmoth, Acherontia 
atropos produces a two-part cry by the movement of air in and out of the pharyngeal cavity (Busnel and Dumortier 1959, Dumortier 1963). The first part of the cry occurs as air moves through the proboscis and past the epipharynx causing it to vibrate. The second part is caused when the epipharynx is raised and the air is forced out through the proboscis. Madagascar hissing cockroaches of the genera Gromphadorhina and Elliptorhina produce sounds by forcing air through an area of modified trachea and a pair of specialized spiracles (Nelson 1979, Sueur and Aubin 2006). Finally, a larval beetle from the genus Cybister, has been reported to produce sound by the expulsion of air through the meso-thoracic spiracles (Mukerji 1929). In A. jugulandis, sounds are produced by forcing air out of the last abdominal spiracle. I propose that the caterpillars close all other spiracles during sound production and the anterior longitudinal contraction forces air through the respiratory system and out the pair of open spiracles.

In the walnut sphinx, I argue that variation in frequency structure between pulse types results from a combination of the size of the spiracle opening and the blowing pressure. Broadband hisses produced by hissing cockroaches occur when the spiracle is completely open, whereas pure whistles, which have different frequency properties, occur when the spiracle is partially closed (Fraser and Nelson 1982). Based on the resemblance of Type 1 and 2 pulses to the hisses, and Type 3 to the whistles of cockroaches, a similar mechanism is possibly being employed. In the walnut sphinx, the pulse amplitude decreases as the train progresses. I suggest that this is a consequence of the rate at which the air passes out of the spiracles as the attack on the caterpillar pauses or ceases. In the hissing cockroach, Nelson (1979) discussed that the amplitude of sounds depends on the rate of air movement as well as other factors that affect air flow, like the opening and 
closing of the spiracle. Further insights into the specific mechanisms underlying sound production in the walnut sphinx include further anatomical and neurophysiological studies of the respiratory system, as well as visualization of respiration using $\mathrm{X}$ ray imaging in live animals (Westneat et al. 2003).

The mechanism of sound production of this species must have been co-opted in some way from respiration. I propose that changes to the respiratory system that resulted in the evolution of sound production included changes to the size, innervation and muscle control over individual spiracles to enable better control over the rate of air flow, as well as modifications to the size of the trachea leading to the spiracle, which would have implications for the resonance and the amount of air being released (c.f. Nelson 1979). I also suggest that the anterior body contractions in the walnut sphinx during sound production are co-opted from telescoping movements used to increase ventilation in some insects (Miller 1974). This hypothesis will be further discussed in Chapter 5.

\section{Function}

Sound production in the walnut sphinx is clearly defensive in nature, since the behaviour is elicited only when attacked. In the following discussion, I argue that the sounds made by walnut sphinx caterpillars function to startle vertebrate predators. To the best of my knowledge, there are no reports describing the predators of walnut sphinx caterpillars. However, I assume that the walnut sphinx is consumed by both vertebrate and invertebrate predators, similar to the predators of other hawkmoth caterpillars that include birds as a major predator, as well as bats, mice, shrews, wasps, ants, beetles and spiders (Pittaway 1993, Tuttle 2007). As with $M$. sexta and $S$. pyri caterpillars, birds, bats, mice and mantids should all be capable of perceiving the sounds produced by $A$. 
juglandis based on their frequency characteristics. Yellow warblers can clearly hear the sounds, based on my results.

I propose that whistles function to startle predators and thereby reduce the risk of predation. My results satisfy the predictions outlined for startle in Chapter 1: (1) They are used only after the prey animal has been disturbed. In support of this, walnut sphinx caterpillars whistled only after being attacked, relying on crypsis as a primary defence. (2) The display is conspicuous, causing the predator to hesitate momentarily, and give the prey an opportunity to escape. The intimidating nature of the sounds produced by walnut sphinx was evident from the reaction of the yellow warblers. Sounds were loud (i.e. conspicuous) and caused the birds to hesitate or abandon their attacks on the caterpillar. In one case, the bird dove away from the caterpillar into thicker vegetation in a manner similar to how it would react to a predator (Gaddis 1980, Lima 1993). Contrary to what is predicted for startle sounds however, none of the caterpillars attempted to escape. This could be explained by the fact that escape is not a viable option for large caterpillars. They cannot run or fly away, and although dropping from the leaf on silk is a common defence strategy for some caterpillars, this has not been observed in the Bombycoidea (Sugiura and Yamazaki 2006). Presumably, large caterpillars do not drop on silk strands because a silk strand cannot support their weight, and dropping to the ground is both energetically costly and dangerous (Dethier 1959). (3) The prey are not otherwise defended, and therefore predators will habituate to the signals. During my attack trials with forceps or birds, there was no evidence that sounds were associated with a chemical defence, and therefore I suggest that sounds do not function primarily as warning sounds. Regurgitation during attack trials was extremely rare - a caterpillar regurgitated only 
once during 18 multi-attack trials, thus differing from the previous studies of sound production in caterpillars (see Chapters 2 and 3). In the absence of a chemical or physical defence, it is argued that attackers will become habituated to the signals, and eventually eat the caterpillar. However, the yellow warblers in this study were clearly deterred by the sounds and did not habituate during the course of the trial sufficiently to return and eat the caterpillar.

Although certain predators may become habituated to startle displays in experimental studies, the type of predator, and the frequency of encountering the display, will dictate how the predator responds (e.g. Bates and Fenton 1990, Vallin et al. 2005, Vallin et al. 2007). In nature, yellow warblers are active foragers, constantly moving through vegetation in their territories in search of food (average territory size across their breeding range varied from 0.04 to 0.39 ha; Lowther et al. 1999). Therefore, if a warbler is deterred from eating a caterpillar during an attack, the same bird is unlikely to encounter the caterpillar again soon afterwards. In addition, foraging time is frequently limited for birds, and they must weigh the costs and benefits of potentially dangerous prey while being vigilant for predators themselves (Kaby and Lind 2003). Thus, I suggest that if foraging yellow warblers encountered prey that produce a startling sound in the wild, then these birds would move on to other food rather than risk a prolonged attack on a potentially dangerous food item.

I conclude that crypsis is the primary defence for walnut sphinx caterpillars, and that following attack, whistle sounds function to startle predators. This hypothesis could be further tested with predator trials on muted caterpillars to determine if sound is sufficient to deter predation, or with predator trials on undefended and palatable food 
items while broadcasting walnut sphinx sounds. Also, I presently cannot rule out chemical defences in the walnut sphinx, though there are no overt signs of chemical defence directly associated with signalling. Walnut sphinx caterpillars feed on some trees that are rich in tannins (e.g. walnut Juglans sp.; beech, Fagus sp.), and these tannins could potentially offer some level of protection to caterpillars. If walnut sphinx also have chemical defences against predators, the whistle sounds may further signal their unprofitability as prey. Further discussion of the function of startle signals in caterpillars, and the evolutionary origins of these signals, will be addressed in Chapter 5. 
CHAPTER 5

Sound Production in Caterpillars: A comparative survey of defensive behaviours in Bombycoidea and other large caterpillars*

*Parts of this thesis are being prepared for publication in 2 manuscripts:

Bura, V.B., Fleming, A.J., Janzen, D.H., Hallwachs, W. and Yack, J.E. Defensive sound production in six species of Bombycoidea caterpillars from Costa Rica (in preparation for submission to Neotropical Entomology)

Bura, V.B., Janzen, D. H. and Yack, J.E. Defensive sounds in Bombycoidea caterpillars: A comparative study (in preparation-journal to be decided upon) 


\subsection{General Introduction}

Acoustic communication in Lepidoptera has been well studied with several reviews on the topic (e.g. Spangler 1988, Scoble 1995, Conner 1999, Miller and Surlykke 2001, Minet and Surlykke 2003, Yack 2004). It is therefore surprising that in all of these reviews there is little or no mention of acoustic communication in the larval stage. As previously outlined in the introduction of Chapter 1, this lack of information is now changing. There is a growing number of examples of caterpillars using both near field sounds and vibrations to detect and/or communicate with heterospecifics and conspecifics, and this list of examples continues to grow each year (see references in Chapter 1). Also, as we have seen in the previous chapters of this thesis, and in recent work by Brown et al. (2007), the production of airborne sounds, which has been overlooked in previous reports on caterpillar anti-predator strategies, has now been documented in two families of Bombycoidea with varying complexity, mechanisms and functions. Given the experimental data presented already, and preliminary field reports on sound production written by naturalists (refer to Table 1.1), I propose that sound production is taxonomically widespread, at least in Bombycoidea caterpillars, and possibly other caterpillars of large size that would be capable of generating these sounds.

Further knowledge of the taxonomic distribution and diversity of airborne sound production in Bombycoidea caterpillars will provide a rich opportunity to study the function and evolution of animal defences, acoustic communication, and predator-prey interactions in caterpillars. Specific questions about sound production in caterpillars include the following: 
Which Bombycoidea species produce sounds and how many times did sound production evolve?

What are the different mechanisms and how did they evolve?

What explains the variation in sound production or lack thereof between caterpillars within and outside of the Bombycoidea?

What are the specific functions of these sounds?

The first step towards answering these questions is to conduct a survey to test which caterpillars produce sounds, how they make sounds and how sound production is associated with other defences. Accordingly, this is the main focus of the final chapter of my thesis, which is divided into three parts. In Part A I will outline a preliminary survey of defensive behaviours in caterpillars of the Bombycoidea, with information on the mechanisms and characteristics of sound production wherever possible. In Part B I will use the information gathered in this thesis to date to pose hypotheses regarding the evolutionary origins of the mechanisms of sound production from non-signalling behaviours. Finally, in Part C I will examine the data to develop hypotheses on the function of sound production and selection pressures and other factors that may help to explain variation in caterpillar sound production. 


\subsection{PART A: Survey of Sound Production and Associated Behaviours}

\subsubsection{Introduction}

To date, all species that have been confirmed (or suggested) to produce airborne sounds belong to the superfamily Bombycoidea and therefore, this is where I have concentrated my efforts to explore sound production in caterpillars. The superfamily comprises more than 3600 species in ten families, the largest of which are the Saturniidae (1900 species) and the Sphingidae (1400 species) (Regier et al. 2008a and references therein, Regier et al. 2008b, Kawahara et al. 2009). These families contain some of the world's largest moths and their species can be found throughout the world, with the highest diversity occurring in the tropics (Pittaway 1993, Lampe 2010). Many species are of great economic importance; some silkmoth cocoons are harvested for the silk industry and their larvae are a food source in many countries (Tuskes et al. 1996, Lampe 2010), while hawkmoths can be important pollinators (Wagner 2005). Due to the vast number of species in these families, the current survey is just the tip of the iceberg, and obviously not intended to represent the entire group, although I did attempt to obtain representatives from as many subfamilies as possible. Obtaining live specimens can be quite difficult and time consuming (e.g. finding gravid females at lights, weather conditions, obtaining permits, etc.), and rearing each species presents different challenges (e.g. finding a source of suitable hostplant, maintaining a clean environment, preventing escapees, and generally trying to lower the mortality level in early instars, etc.). In some cases only a few individuals, or even a single individual, could be obtained. If sound was discovered in any of these 'low n' species it was counted as a positive result, but if that individual 
did not produce sound it is not necessarily a negative result. Despite these limitations, I endeavoured to, and was successful in obtaining several different species from North America, Europe and Central America. In Costa Rica the opportunity arose to test large caterpillars of comparable size to those of the Bombycoidea, but that belonged to other superfamilies (e.g Noctuoidea, Papilionoidea, Lasiocampoidea), so I tested, at random, anything that was available to me.

\subsubsection{Methods}

Animals

Bombycoidea caterpillars were obtained from a variety of sources and chosen based on their availability (Table 5.1). Larvae obtained in Costa Rica were identified by Dr. Dan Janzen or one of his staff members (final identifications are obtained by barcoding the DNA of adult specimens, all information is used in an ongoing biodiversity database that began in 1977 and is available at <http://janzen.sas.upenn.edu/caterpillars/ database.lasso $>$, reference numbers are provided in Appendix I) and housed in individual bags filled with hostplant at one of four sites in the Area de Concervación Guanacaste; Area Administrativa (1050'15.49”N, 85³7’07.36”W), Estacion San Gerardo

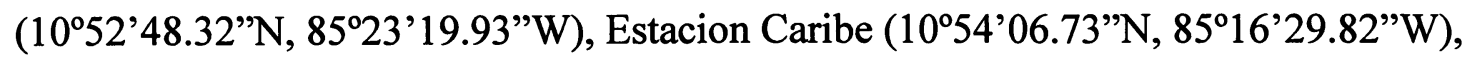
and La Perla $\left(10^{\circ} 46^{\prime} 02.24^{\prime \prime N}, 8^{\circ} 25^{\prime} 58.58^{\prime \prime W}\right)$. Caterpillars of three species were found on vegetation by the side of the road, Citheronia lobesis, Eumorpha satellitia, and Crinodes besckei, and were subsequently identified by Dr. Janzen. All species collected in Costa Rica were tested on site. 
Table 5.1 List of species examined showing the origin of the specimen, the food plant they were reared on, the number of individuals tested, and the date tested.

\begin{tabular}{|c|c|c|c|c|c|c|}
\hline Family & Subfamily & Species & Origin & Food Plant & $\begin{array}{c}\text { Number } \\
\text { Tested }\end{array}$ & $\begin{array}{c}\text { Date } \\
\text { Tested }\end{array}$ \\
\hline Bombycidae & Apatelodinae & Olceclostera angelica (Grote) & eggs from wild caught female at QUBS ${ }^{\top}$ & Fraxinus sp., Syringa sp. & 6 & Aug-09 \\
\hline \multirow[t]{15}{*}{ Saturniidae } & Ceratocampinae & Anisota virginiensis (Drury) & eggs from wild caught female at QUBS & Quercus sp. & 12 & Aug-09 \\
\hline & & Citheronia lobesis Rothschild & Costa Rica, on the way to Playa Naranjo & $\begin{array}{l}\text { Cochlospermum } \\
\text { vitifolium }\end{array}$ & 2 & Jun-09 \\
\hline & & Dryocampa rubicunda (Fabricius) ${ }^{2}$ & $\begin{array}{l}\text { eggs from wild caught females at Mer } \\
\text { Bleue (2006); Bill Oehkle }{ }^{3}(2009)\end{array}$ & Acer saccharum & 27 & Aug-09 \\
\hline & & Eacles imperialis (Drury) & eggs from wild caught female at QUBS & Pinus sp., Picea sp. & 11 & $\begin{array}{c}\text { Jul-09 } \\
\text { /Aug-09 }\end{array}$ \\
\hline & & Eacles ormondei Schaus & Costa Rica, Dr. Dan Janzen & Tapirira mexicana & 1 & Jun-09 \\
\hline & & Schausiella santarosensis Lemaire & Costa Rica, house of Dan Janzen & Hymenaea courbaril & 3 & Jun-09 \\
\hline & Hemilucinae & Automeris postalbida Schaus & Costa Rica, Dr. Dan Janzen & Acalypha diversifolia & 1 & Jun-09 \\
\hline & & Automeris io (Fabricius) & $\begin{array}{l}\text { Bill Oehlke, eggs from wild caught females } \\
\text { at QUBS }\end{array}$ & $\begin{array}{l}\text { Betula papyrifera, Acer } \\
\text { sp., Populus sp. }\end{array}$ & 20 & $\begin{array}{l}\text { Jul-07 } \\
/ \text { Jul-09 }\end{array}$ \\
\hline & & Automeris phrynon Druce & Costa Rica, Dr. Dan Janzen & Terminalia catappa & 1 & Jun-09 \\
\hline & Saturniinae & Actias luna (Linnaeus) ${ }^{4}$ & $\begin{array}{l}\text { eggs from wild caught females at Mer } \\
\text { Bleue (2005); eggs from wild caught } \\
\text { females at QUBS ( } 2009, \text { morphology only) }\end{array}$ & Betula papyrifera & 10 & Jul-05 \\
\hline & & $\begin{array}{l}\text { Antheraea pernyi (Guérin- } \\
\text { Méneville) }\end{array}$ & donated by Mario Ioppolo (Italy) & Quercus sp. & 2 & May-07 \\
\hline & & Antheraea polyphemus (Cramer) ${ }^{4}$ & $\begin{array}{l}\text { eggs from wild caught females at Mer } \\
\text { Bleue or East Bridgewater MA (USA); Bill } \\
\text { Oehlke (2005); eggs from wild caught } \\
\text { females at QUBS ( } 2009, \text { morphology only) }\end{array}$ & $\begin{array}{l}\text { Quercus rubra, Betula } \\
\text { papyrifera, Acer } \\
\text { saccharum }\end{array}$ & 150 & Jul-05 \\
\hline & & Attacus atlas (Linnaeus) $^{5}$ & Worldwide Butterflies (P-2004-02683) & Citrus sp. & 8 & Dec-06 \\
\hline & & Callosamia promethea (Drury) ${ }^{2}$ & $\begin{array}{l}\text { Bill Oehlke (2006 \& 2009); eggs from wild } \\
\text { caught female at QUBS (2009) }\end{array}$ & Betula sp. & 34 & $\begin{array}{c}\text { Jul-09 } \\
\text { /Aug-09 }\end{array}$ \\
\hline & & $\begin{array}{l}\text { Saturnia pyri (Denis \& } \\
\text { Schiffermüller) }\end{array}$ & $\begin{array}{l}\text { Bernhard Wenczel, Denmark (2007, P- } \\
\text { 2007-03105); Rolf Schafroth, Switzerland } \\
(2008, \text { P-2008-02614) }\end{array}$ & Populus sp. & 52 & $\begin{array}{l}\text { Jun-07 } \\
\text { /Jun-08 }\end{array}$ \\
\hline
\end{tabular}


Table 5.1 continued

\begin{tabular}{|c|c|c|c|c|c|c|}
\hline Family & Subfamily & Species & Origin & Food plant & $\begin{array}{c}\text { Number } \\
\text { Tested }\end{array}$ & $\begin{array}{l}\text { Date } \\
\text { Tested }\end{array}$ \\
\hline \multirow[t]{19}{*}{ Sphingidae } & Smerinthinae & $\begin{array}{l}\text { Adhemarius ypsilon (Rothschild \& } \\
\text { Jordan) }\end{array}$ & Costa Rica, Dr. Dan Janzen & Ocotea atirrensis & 1 & Jun-09 \\
\hline & & Amorpha juglandis (JE Smith) & eggs from wild caught female at QUBS & $\begin{array}{l}\text { Ostyra sp., Alnus sp., } \\
\text { Juglans sp., Fagus sp. }\end{array}$ & 18 & $\begin{array}{l}\text { Jul-08 } \\
\text { /Aug-09 }\end{array}$ \\
\hline & & Mimas tiliae (Linnaeus) ${ }^{4}$ & donated by Jake Miall (Europe) & Alnus sp. & 3 & Jul-05 \\
\hline & & Paonias excaecatus (JE Smith) ${ }^{7}$ & $\begin{array}{l}\text { eggs from wild caught females at QUBS } \\
\text { (2008); Bill Oehkle (2009) }\end{array}$ & Betula sp. & 14 & $\begin{array}{c}\text { Jul-09 } \\
\text { /Aug-09 }\end{array}$ \\
\hline & & Paonias myops (JE Smith) & eggs from wild caught female at QUBS & Prunus sp. (cherry) & 20 & Jul-09 \\
\hline & & Smerinthus cerisyi Kirby ${ }^{4}$ & $\begin{array}{l}\text { eggs from wild caught females at Mer } \\
\text { Bleue }\end{array}$ & Populus tremuloides & 10 & Jul-05 \\
\hline & & Smerinthus jamaicensis (Drury) ${ }^{5}$ & $\begin{array}{l}\text { eggs from wild caught females at Mer } \\
\text { Bleue }\end{array}$ & Populus tremuloides & 5 & Jul-06 \\
\hline & Sphinginae & Acherontia atropos (Linnaeus) & Worldwide Butterflies (P-2004-02683) & Nightshade & 5 & Jun-07 \\
\hline & & Ceratomia undulosa (Walker) & eggs from wild caught female at QUBS & Fraxinus sp. & 20 & Jul-09 \\
\hline & & Manduca albiplaga (Walker) & Costa Rica, Dr. Dan Janzen & Rollinia membranacea & 1 & Jun-09 \\
\hline & & Manduca florestan (Stoll) & Costa Rica, Dr. Dan Janzen & $\begin{array}{l}\text { New species (\#19080) } \\
\text { (Bignoniaceae) }\end{array}$ & 1 & Jun-09 \\
\hline & & $\begin{array}{l}\text { Manduca pellenia (Herrich- } \\
\text { Schaffer) }\end{array}$ & Costa Rica, Dr. Dan Janzen & Cestrum megalophyllum & 1 & Jun-09 \\
\hline & & Manduca sexta (Linnaeus) & $\begin{array}{l}\text { Dalhousie University (2005), Livefood } \\
\text { Mercier QC (2006), Canadian Feeders } \\
\text { Windsor ON (2008) }\end{array}$ & $\begin{array}{l}\text { Solanum lycopersicum, } \\
\text { Datura inoxia, Nicotiana } \\
\text { tabacum, Artificial diet }\end{array}$ & $62+$ & May-08 \\
\hline & & Sphinx chersis (Hubner) & $\begin{array}{l}\text { early instar caterpillar found on ash tree at } \\
\text { Carleton University }\end{array}$ & Fraxinus sp. & 1 & Jul-09 \\
\hline & & Sphinx drupiferarum (JE Smith) & Bill Oehlke & Prunus sp. (plum) & 16 & Aug-09 \\
\hline & & Sphinx kalmiae (JE Smith) & Bill Oehlke & Fraxinus sp. & 20 & Jul-09 \\
\hline & & Sphinx poecila Stephens & Bill Oehlke & Vaccinium sp. & 18 & Aug-09 \\
\hline & Macroglossinae & Darapsa choerilus (Cramer) & Bill Oehlke & Viburnum sp. & 5 & Jul-09 \\
\hline & & Darapsa myron (Cramer) & $\begin{array}{l}\text { caterpillar found eating grape on Carleton } \\
\text { campus and at Carleton land (Manotick) }\end{array}$ & Vitis $s p$. & 1 & Aug-09 \\
\hline
\end{tabular}


Table 5.1 continued

\begin{tabular}{|c|c|c|c|c|c|c|}
\hline Family & Subfamily & Species & Origin & Food plant & $\begin{array}{c}\text { Number } \\
\text { Tested }\end{array}$ & $\begin{array}{c}\text { Date } \\
\text { Tested }\end{array}$ \\
\hline \multirow[t]{7}{*}{ Sphingidae } & Macroglossinae & Deidamia inscriptum (Harris) & $\begin{array}{l}\text { last instar caterpillar found while beating } \\
\text { grape at QUBS }\end{array}$ & Vitis $s p$ & 1 & Jun-09 \\
\hline & & Eumorpha satellitia (Linnaeus) & Costa Rica, Dr. Dan Janzen & Vitis tiliifolia & 2 & Jun-09 \\
\hline & & Nyceryx magna (Felder) & Costa Rica, Dr. Dan Janzen & $\begin{array}{l}\text { Pentagonia donnell- } \\
\text { smithii }\end{array}$ & 1 & Jun-09 \\
\hline & & $\begin{array}{l}\text { Pachygonidia drucei (Rothschild \& } \\
\text { Jordan) }\end{array}$ & Costa Rica, Dr. Dan Janzen & Doliocarpus multiflorus & 1 & Jun-09 \\
\hline & & Sphecodina abbottii (Swainson) & $\begin{array}{l}\text { found as larvae in Ottawa ON, by } \\
\text { construction worker (2007) and Trisha } \\
\text { Gahan (2008) }\end{array}$ & Vitis sp. & 3 & Jul-08 \\
\hline & & Xylophanes anubus (Cramer) & Costa Rica, Dr. Dan Janzen & Psychotria panamensis & 1 & Jun-09 \\
\hline & & Xylophanes tyndarus (Boisduval) & Costa Rica, Dr. Dan Janzen & Faramea occidentalis & 2 & Jun-09 \\
\hline \multirow[t]{4}{*}{ Nymphalidae } & Morphinae & Antirrhea lindigii Felder & Costa Rica, Dr. Dan Janzen & Geonoma ferruginea & 1 & Jun-09 \\
\hline & & Morpho amathonte Deyrolle & Costa Rica, Dr. Dan Janzen & Lonchocarpus oliganthus & 1 & Jun-09 \\
\hline & Nymphalinae & Smyrna blomfildia (Fabricius) & Costa Rica, Dr. Dan Janzen & Urera caracasana & 1 & Jun-09 \\
\hline & Danainae & Danaus sp. & Costa Rica, Dr. Dan Janzen & Solandra grandiflora & 1 & Jun-09 \\
\hline Lasiocampidae & Macromphaliinae & Euglyphis sp. & Costa Rica, Dr. Dan Janzen & Cinnamomum brenesii & 1 & Jun-09 \\
\hline Hesperiidae & Eudaminae & Astraptes creteus crana Evans & Costa Rica, Dr. Dan Janzen & Styrax argenteus & 1 & Jun-09 \\
\hline \multirow[t]{3}{*}{ Notodontidae } & unknown & Lirimiris fascis & Costa Rica, Dr. Dan Janzen & $\begin{array}{l}\text { Mortoniodendron } \\
\text { guatemalense }\end{array}$ & 1 & Jun-09 \\
\hline & & Lirimiris guatemalensis Rothschild & Costa Rica, Dr. Dan Janzen & Guazuma ulmifolia & 1 & Jun-09 \\
\hline & Dudusinae & Crinodes besckei (Hübner) & Costa Rica, Dr. Dan Janzen & Gouania sp. & 1 & Jun-09 \\
\hline \multirow[t]{2}{*}{ Noctuidae } & Catocalinae & Acanthodica sinuilinea Prout & Costa Rica, Dr. Dan Janzen & Heteropterys obovata & 1 & Jun-09 \\
\hline & unknown & unknown species & Costa Rica, Dr. Dan Janzen & Dioscorea urophylla & 1 & Jun-09 \\
\hline
\end{tabular}

TQUBS - Queen's University Biological Station, Lake Opinicon, ON

${ }^{2}$ Species re-tested by me (originally by Antoine Hnain), only information obtained from my trials is included in the analyses presented here

${ }^{3}$ Eggs were purchased from wild females caught by Bill Oehkle. Moths were captured in Ontario and Prince Edward Island

${ }^{4}$ Species tested by Sarah Brown only, video footage analysed by me

${ }^{5}$ Species tested by A. Hnain only, video footage analysed by me

${ }^{6}$ Canadian Food Inspection Agency import permit numbers

${ }^{7}$ Species re-tested by me (originally by Justin Hick), only information obtained from my trials is included in the analyses presented here 
All other species were reared and housed in an insect rearing room at Carleton University in species-specific jars or plastic bins containing cuttings of the appropriate food plant. Caterpillars were exposed to a 16:8 hour light:dark cycle and kept at approximately $25^{\circ} \mathrm{C}$. Food plants were replenished as needed, usually once every 1-3 days per species. A photograph was taken of the larvae and adult females (when applicable) for each species to aid in species identification. Species that were purchased from companies or collected by individuals were identified by the source, and further confirmed in late instars using field guides and reference books (e.g. Covell 2005, Wagner 2005, Tuttle 2007). In both locations (Carleton University and Costa Rica) voucher specimens were kept whenever possible by first sacrificing the larva in boiling water and subsequently placing it in a vial of $70 \%$ ethanol. Voucher specimens are stored at Carleton University in the Yack laboratory.

\section{Attack experiments}

Individual late instar $\left(4^{\text {th }}\right.$ or $\left.5^{\text {th }}\right)$ caterpillars were placed on sprigs of host plant and isolated for at least 30 minutes prior to experimentation whenever possible. Head capsule widths were measured with a ruler to identify the instar of the caterpillar. Larvae were tested for sound production by delivering multiple pinches to the head capsule or posterior end using blunt forceps to simulate the attack of a predator (e.g. Bowers 2003, Grant 2006), with approximately 5 seconds between consecutive pinches. Behavioural responses were recorded for at least 60 s following the first pinch with a Sony handycam (DCR-HC85 or HDR-HC7, Tokyo, Japan) equipped with a Sony ECM microphone (ECM-MS907 or ECM-MS957, Tokyo, Japan) placed approximately 2-5 cm away from 
the head capsule of the larvae. Recordings at Carleton University were performed indoors in an enclosure lined with acoustic foam. Recordings in Costa Rica were performed outdoors at one of the research stations mentioned above, in a portable collapsible insect/reptile cage lined with acoustic foam. Trials were reviewed in QuicktimePro 7.6.5 and analysed for the occurrence of sound production and other defensive behaviours (see Results). In a few cases I analyzed previously collected video footage obtained by previous members of the Yack lab (S. Brown and A. Hnain) to gather further information about the defensive behaviours of particular species. These cases are noted in Table 5.1.

\section{Sound recording and analysis}

When sound production was discovered in a species, further recordings were performed to characterize their signals temporally and spectrally. Individual caterpillars were placed on a cutting of hostplant and stimulated to signal by delivering a pinch with blunt forceps to either the head capsule or body region. Sounds were recorded with a Brüel \& Kjær (Naerum, Denmark) 1/4"microphone type 4939, placed approximately 5 or $10 \mathrm{~cm}$ away from the caterpillar, amplified with a Brüel \& Kjær Nexxus conditioning amplifier type 2690, and recorded onto a Fostex FR-2 Field Memory Recorder (Gardena, CA, USA) at a sampling rate of $192 \mathrm{kHz}$. All recordings at Carleton University were performed in an acoustic chamber (Eckel Industries Ltd., Cambridge, MA, USA). In Costa Rica, specimens were brought indoors to perform the recordings inside the portable acoustic enclosure to decrease noise. Recordings were later analyzed using RavenPro Bioacoustics Research Program 1.4 (Cornell Laboratory of Ornithology, Ithaca, NY, USA) and AviSoft SASlab Pro (Avisoft Bioacoustics, Berlin, Germany). Information for 
A. polyphemus and A. luna was taken from Brown (2006) and Brown et al. (2007).

Temporal characteristics, including train duration, number of sound pulses in a train, pulse duration and number of components in a pulse, were measured from as many recordings as possible per species (see Results for specific n's). A train was defined as a series of sound pulses following an attack until sound production ceased (Fig 5.1a). Individual sound pulses were defined as the smallest unit of sound distinguishable by the unaided human ear (Fig 5.1b) and were named differently for each mechanism with respect to how they sounded to the human ear (i.e. click, chirp, rasp, whistle, see Results). Pulse components are equivalent to toothstrikes or impulses (Ewing 1989), and make up a pulse (Fig 5.1c).

Spectral characteristics including dominant frequency and quality factors Q3 and Q10 (Fig 5.1d; the dominant frequency divided by the frequency bandwidth at -3 and -10 $\mathrm{dB}$, Ewing 1989) were measured from as many recordings as possible per species (see Results for specific n's). Power spectra were produced using a 512-point Fast Fourier Transform (Hann window).

\section{Head capsule and mandible morphology}

When voucher specimens could be obtained (this was not possible for all of the Costa Rican species), mandibles from one specimen were removed, cleaned with an ultrasonic cleaner (Bransonic B200, Branson Ultrasonic Corporation, Danbury, CT) and photographed using an Olympus SZX12 (Olympus Corporation, Tokyo, Japan) light 
a

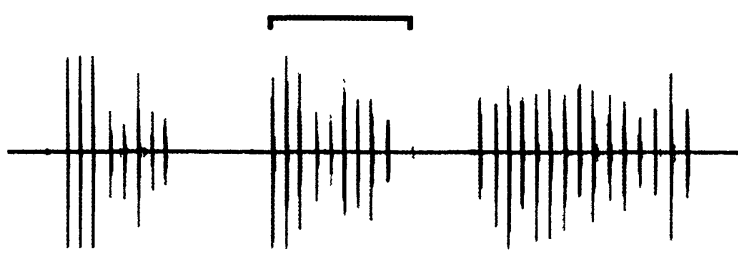

$\overline{1 s}$

b

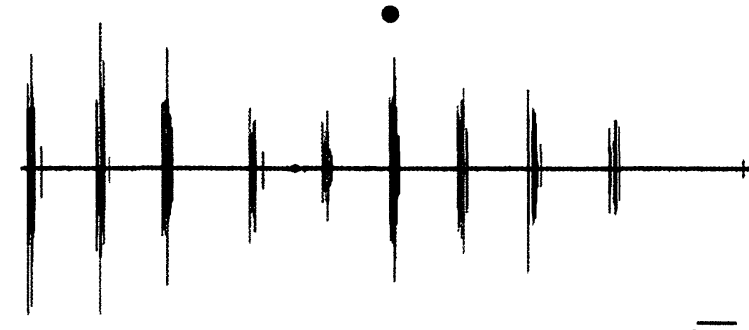

C

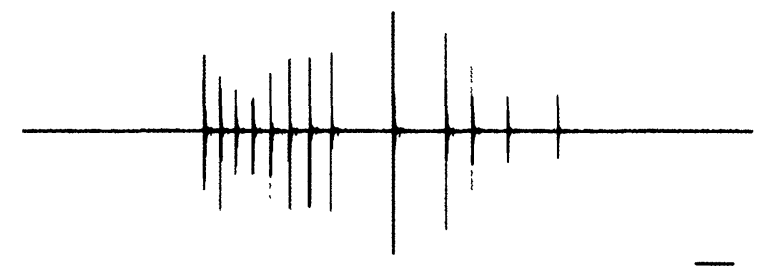

d

$0 . \overline{005} \mathrm{~s}$

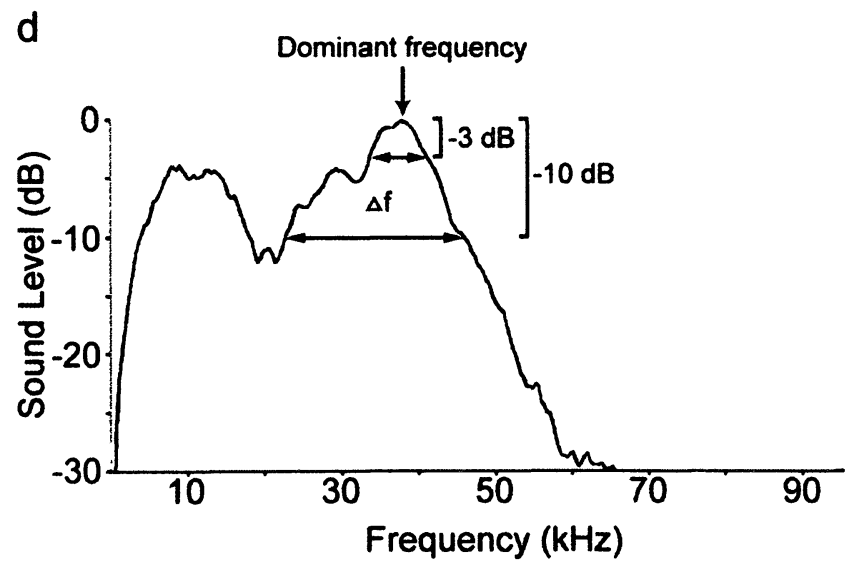

Figure 5.1 Representative trace from a sound-producing caterpillar displaying characteristics measured for analysis. (a) Oscillograms of three trains of sounds. (b) Expanded time scale of one train (bracket in a) showing 10 sound pulses. (c) Expanded time scale of one pulse (dot in b) showing 13 components. (d) Power spectrum with dominant frequency and frequency bandwidth $(\Delta \mathrm{f})$ at -3 and $-10 \mathrm{~dB}$. 
microscope equipped with an AxioCam MRc5 (Carl Zeiss Micro Imaging GmbH, Göttingen, Germany) digital camera. Images were captured using AxioVision 4.6. Excised mandibles were sputter-coated with gold-palladium and examined using a VEGA II XMU variable pressure scanning electron microscope (Tescan USA Inc., Cranberry Twp., PA). Head-capsule measurements were taken from images acquired with the microscope setup above from 1-5 fixed specimens per species. Length, width and area were measured from each image using ImageJ 1.42q (National Institutes of Health, USA). The shape of the head was described as rounded, oval or triangular, based on visual impressions and the difference between length and width of the head-capsule (i.e. if the average head width - head length $<-0.1$, the head was described as being oval).

\subsubsection{Results and Discussion}

Species accounts

To date, 60 species have been tested or noted in the literature for their ability to produce sounds: 49 from Bombycoidea (2 Bombycidae, 18 Saturniidae, 29 Sphingidae); 5 from Noctuoidea (3 Notodontidae, 2 Noctuidae); 4 from Papilionoidea (2 Morphinae, 1 Nymphalinae, 1 Danainae); 1 from Lasiocampoidea (Lasiocampidae) and 1 from Hesperioidea (Hesperiidae). During the course of my thesis, I tested 47 species directly (36 Bombycoidea and 11 outgroups), and analyzed tapes of an additional 6 species from previous students (all Bombycoidea, noted in Table 5.1). Detailed information on these 53 species is provided in the following species accounts. For each species, I have listed their geographical distribution and notable morphological and behavioural characteristics 
associated with sound production and/or other defences. For those species that produced sound, additional information is provided on the sound characteristics, proposed mechanisms, and relationship to other defences. Before presenting these species accounts, I provide brief summaries of the observed behaviours, as well as a 'roadmap' to explain how sounds, behaviours, and morphology were categorized or described. The data provided in these sheets will be discussed again at the end of this section, where sound production is mapped onto phylogenies to answer questions about taxonomic distribution, as well as in sections $\mathrm{B}$ and $\mathrm{C}$ of this chapter, which focus on evolutionary origins and possible functions. Please note that the 7 species missing from these sheets were either tested by a former student and did not have tapes that could be reviewed, or the information came strictly from the literature. All 60 species are included in the summary table at the end of this section (Table 5.2).

\section{Defensive behaviours}

A study of the defensive behaviours described in the following species accounts of the 42 Bombycoidea and 11 outgroup species revealed an interesting diversity of behaviours that occurred following a simulated attack. In some cases individuals would respond differently to attacks with forceps delivered to the anterior or posterior body regions, which is also noted in the species accounts. After reviewing footage I determined that most defensive behaviours could be classified into 12 general categories which are outlined below. However, as these categories are based on simulated attacks other defences may exist when the animal is confronted by a natural predator. 
Sound Production

In the 42 species of Bombycoidea that I studied, 14 made acoustic signals when disturbed, and one other clearly made sounds but it was uncertain whether or not these sounds were incidental or a true signal. These 'borderline' signals, and how to distinguish them from true communication signals will be discussed further in Part B of this chapter. Oscillograms are presented at approximately the same time scale for comparative purposes. Of the 11 outgroup species, all of which were of equivalent size to the average Bombycoidea tested, none produced sounds. After reviewing all species I studied, I have categorized the observed sounds into 4 general types: clicking, stridulation, rasping and whistling.

Clicking describes sharp, short-sounding sounds produced by the mandibles, and resembles the sounds made by clicking the fingernails of the thumb and finger together. The mechanism was confirmed in some species using video analysis but mandible movements were also visible with the unaided eye. Clicks have been found in 8 species and can occur individually or in trains, which can be long in duration for some species (Table 5.3). One click is equivalent to one pulse, produced by a single movement and contains a minimum of one component. Mandible clicking is considered a type of stridulation using undefined parts (Dumortier 1963) and is also used by other insects, such as grasshoppers, in conspecific communication and as a response to disturbance (Alexander 1960, Blondheim and Frankenberg 1983).

Stridulation occurs when the edge of one mandible rubs against the inside of the other, producing pulsed sounds, or chirps, that are similar to running something hard through the teeth of a plastic comb. As with clicking, the mechanism was confirmed in 
some species using video analysis but the movements could also be seen with the unaided eye. Chirps have been found in three species and occurred singly or in short or long trains (Table 5.3). Though individual chirps, like clicks, are also equivalent to individual pulses and are created by a single movement of the mandibles, one chirp typically contained more components. Since the mandibles of these caterpillars do not possess a defined file and scraper, mandible stridulation is also stridulation using undifferentiated parts. I am aware of two examples of orthopteran insects using undefined elytral stridualtion for conspecific communication, one in a katydid and the other in a grasshopper (Dumortier 1963 and references therein).

Rasping sounds are labelled as such based on terminology used by Heinrich (1979) to describe the sounds of the Abbott's sphinx caterpillar; 'crackling-rasping'. Rasps have been found in three species (Table 5.3); in two species the rasps sometimes sound like squeaks and other times like the balls of a Newton's cradle; in the third species they sound like an electric discharge. Sounds are produced when the mandibles are open and wide apart; I suspect the passage of air causes the vibration of a membrane somewhere in the foregut or buccal cavity of the caterpillar. This mechanism has not yet been confirmed though the opening of the mandibles can be seen using video analysis and the unaided eye. Rasps typically occur in trains, sometimes followed by a series of clicks, though they can occur alone. One rasp is equivalent to one pulse and usually contains more than two components. Since the mechanism is unconfirmed the existence of other examples is questionable at this time, however, if rasps are produced by the movement of air over a membrane, they may resemble the inspiration part of the cry of adult $A$. atropos (see Part $\mathrm{B}$ for more details). 
Whistling describes the sounds produced by the passage of air through a pair of abdominal spiracles and resembles the noise produced by a squeaky toy. The mechanism was confirmed using selective occlusions and laser vibrometry (described in Chapter 4) and has been found in one species (the walnut sphinx). Whistles are typically produced in trains but can occur alone. A single whistle is equivalent to a single pulse and is elicited by one longitudinal contraction. The production of sound by the movement of air is rare in insects, but has been noted in Madagascar hissing cockroaches and at least one species of beetle (Mukerji 1929, Nelson 1979, Sueur and Aubin 2006). Whistles likely also resemble the sounds produced during the expiration part of the cry of adult $A$. atropos (Busnel and Dumortier 1959).

Regurgitation

Regurgitation occurs when a caterpillar releases viscous fluid out through the mouth, and has been shown to be an effective defence against vertebrate and invertebrate predators (Grant 2006, Rostás and Blassmann 2009 and references therein). According to Grant (2006) there are three ways in which caterpillars use regurgitation as a defence: Primary regurgitators regurgitate soon after attack, can control the amount released and the direction it is aimed in, and re-imbibe the bubble when attacks cease. Secondary regurgitators will regurgitate after thrashing, biting or trying to escape, cannot control the amount released and do not re-imbibe. Finally, non-regurgitators will regurgitate only after long lasting attacks (if at all) and may be a result of exhaustion or stress rather than a true defence. Twenty-seven species regurgitated (64.3\%) either at some point during the attack trials or when being handled. Sometimes it was clearly directed at the attacking 
forceps or maintained as a bubble (e.g. X. anubus, A. polyphemus), while other times it was released in vast quantities after many pinches (e.g. $P$. drucei), while still others did not regurgitate at all (e.g. A. virginiensis). The temporal relationship between regurgitation and sound production is important in understanding the function of defensive sounds (i.e. does sound precede, accompany or follow regurgitation) and this is noted, where possible, on the sheets of individual species. This relationship was easier to document in species where there was a large number of individuals and so may not be summarized in 'low n' species as the one specimen may not have displayed both behaviours.

Chemical defence

A chemical defence was considered separate from regurgitation and was described by the presence of scoli or a strong odour. Three types of scoli were observed in the Saturniidae, urticating trees, secretory and spraying scoli. The two former types are effective injection devices for defensive compounds produced by the caterpillars, while the latter is capable of spraying compounds into sensitive eyes or mucous membranes of vertebrate predators (Deml and Dettner 2002).The chemical compounds produced by these caterpillars has been shown to be effective against a host of different predators including fungi, ants and birds (Deml and Dettner 1995). Chemical bearing scoli were observed in 5/42 Bombycoidea species, and a strong smelling odour was observed in 1 outgroup species. When sound was also observed in any of these species the relationship between the onset of signalling and the secretion of chemicals was noted, as for regurgitation. 
Thrashing

This heading encompasses three behaviours listed separately on the plates, thrashing, major thrashing and directed thrashing. Thrashing is a series of side to side movements where the caterpillar would release a few prolegs and swing its body in an arc from side to side. It occurred at various rates, from a struggled wriggling-like motion (e.g. S. kalmiae) to a high-paced wide arc at a rate of about 2 side movements per second (i.e. major thrashing, e.g. A. ypsilon and N. magna). Low to regular paced thrashing occurred in 18 species while major thrashing was observed in 6 species. Directed thrashing was different and involved the caterpillar swinging its anterior body once either towards or away from the site of attack (depending on if it was attacked posteriorly or anteriorly). This was by far the most common reaction to attack, occurring in $38 / 42$ species. Thrashing may stunt successful attacks by avian predators (Walters et al. 2001) and has been shown to be effective against parasitoids, coccinellids and ants (Stamp 1986, Deml and Dettner 1993, Gentry and Dyer 2002). Directed thrashing allows the caterpillar to defend the immediate area surrounding it with other behaviours (Stamp 1986) like biting or regurgitation.

Biting

Biting occurred as the caterpillars thrashed towards the part of the forceps that made contact with their bodies. This could be seen from the videos as mandibles being held open wide while in a straight position and closing them after thrashing. Ten species exhibited this behaviour. Biting has been shown to be effective against coccinellids and 
paratitoid wasps (Stamp 1986, Gentry and Dyer 2002). This behaviour was also observed while handling the caterpillars and in some cases was quite forceful.

\section{Curling}

This heading encompasses two defences listed in the sheets: curling-in and curling-up. In the former, the caterpillar would slightly curl the anterior portion of its body and hide its head in its ventral side. This behaviour was often accompanied by regurgitation (69.2\%). An extreme version of curling-in occurs in certain species of the subfamily Macroglossinae wherein the caterpillar is able to retract its head and thoracic segments into its abdomen. In total, 26/42 species exhibited this behaviour, especially on attacks directed at the anterior end. Curling-up occurred when the caterpillar curled its anterior end toward its posterior end, resembling a circle, ball or corkscrew. This behaviour was only seen in two species $(4.8 \%)$. In species with protective scoli curling-in or up is a way to present the scoli so that more area is protected (Deml and Dettner 1995). This has been shown to be effective against ants (Deml and Dettner 1993). In species without this protection, curling-in or up could draw attention to other morphological features. For example, some macroglossines that retract their head and thorax into their abdomen possess eye-spots that become more prominent with this behaviour (e.g. $X$. tyndarus). Alternatively, if there are no obvious morphological features, curling-in or up may simply make it more difficult for a successful attack on the head-capsule.

Twitching 
Twitching involved the caterpillar longitudinally contracting its body in rapid bursts occasionally causing small movements dorsally. Nine species exhibited this behaviour (21.4\%). Twitching may be similar to telescoping movements used by some insects to increase ventilation in preparation for increased activity (Miller 1974), and may serve a similar function in preparing for other behaviours such as thrashing or regurgitating. This will be discussed further in Part B of this chapter.

\section{Walking away}

Occasionally a caterpillar would simply walk away from the attack, leaving the leaf where it was staged (4/42 species, 9.5\%). This either occurred between successive pinches or once the pinches had stopped. This type of escape behaviour has been noted in simulated attacks with pipevine caterpillars (Stamp 1986), though its effectiveness with a natural predator is questionable, unless the caterpillar was able to flee very quickly.

\section{Dropping}

A few species would release all their prolegs and drop to the ground once an attack was delivered (4/42 species, 9.5\%). Once on the ground a few different behaviours were observed and are noted in the individual sheets. Dropping from a hostplant can be dangerous for a caterpillar (Dethier 1959) and many that do drop will hang from a silk thread, or lifeline, though this has not been observed in Bombycoidea (Sugiura and Yamazaki 2006). Dropping can be an effective defence against parasitoid wasps and coccinellids (Stamp 1986, Gentry and Dyer 2002) though it is unclear if the caterpillar would make it back to its plant. In Santa Rosa National Park, the only bombycoids that 
were observed to fall from their hostplant were species that were very polyphagous and would likely have little trouble finding another suitable host (Janzen 1984).

\section{Mandible morphology}

Since two of the four sound categories use the mandibles as the source of sound production I examined mandible structure from all species whenever possible. In total 33 sets of mandibles were photographed, leading to three general classifications, smooth, serrated, and ridged and serrated, inspired from the descriptions by Bernays and Janzen (1988). Smooth mandibles had an anterior edge that lacked any obvious teeth and had no serrated ridges on their inner or outer faces (though occasionally there was a single blunt ridge on the inner face). Serrated mandibles also lacked ridges on their inner and outer faces but the anterior edge was noticeably toothed or serrated. Ridged and serrated mandibles had a serrated anterior edge and possessed serrated ridges on either or both of the inner and outer face. Topographic nomenclature of the mandibles was described after Bernays and Janzen (1988) and is illustrated in Fig 5.2. 


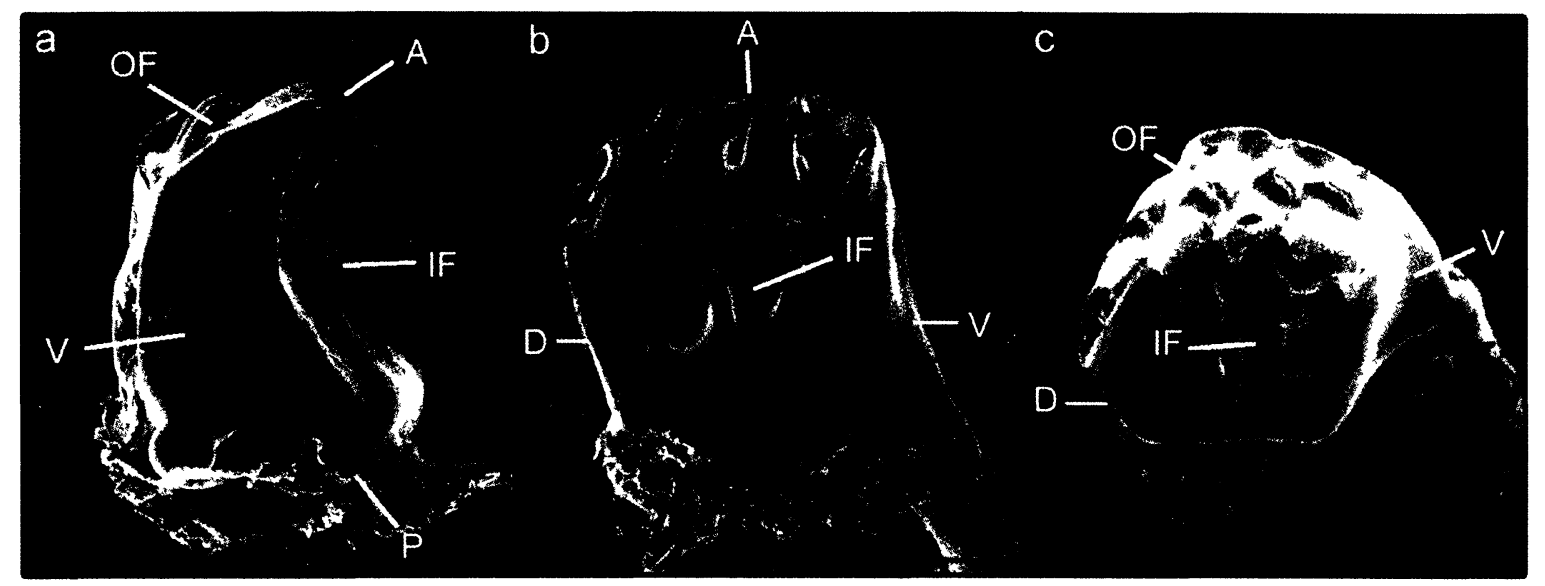

Figure 5.2 Topographic nomenclature of mandible morphology. This specimen was taken from Manduca sexta (Sphingidae), and illustrates and example of ridged and serrated mandibles (a) Ventral-anterior view of a right mandible, A- anterior edge, OF - outer face, IF - inner face, V ventral side. $P$ posterior side, scale bar $0.25 \mathrm{~mm}$. (b) Inner face view of left mandible, $D$ dorsal side, scale bar $0.25 \mathrm{~mm}$. (c) Antero-posterior view of left mandible, scale bar $0.25 \mathrm{~mm}$. 


\section{SUPERFAMILY: BOMBYCOIDEA}

\section{FAMILY: BOMBYCIDAE}

The family Bombycidae is a tentative grouping of at least 190 species in four subfamilies, Apatelodinae, Bombycinae, Phiditiinae and Prismostictinae (Regier et al. 2008a). The relationships among the traditional groupings were not supported in a recent study and the four subfamilies may be more closely related to other groups (Regier et al. 2008a).

\section{SUBFAMILY: APATELODINAE}

\section{Olceclostera angelica (Grote), the Angel, Fig 5.3}

The Angel is a new world moth found throughout eastern North America (Wagner 2005). Caterpillars of this species feed on ash (Fraxinus sp.) and lilac (Syringa sp.), both members of the Oleaceae or olive family (Wagner 2005).

Morphology: Late instar $O$. angelica caterpillars are very hairy, have black tufts of hair on the dorsal side of each abdominal segment and a partially concealed patch of red on the dorsal side of their second thoracic segment (Fig $5.3 \mathrm{a}, \mathrm{b}$ ). At the time of experimentation the fifth instar of this species was $4 \mathrm{~cm}$ in length, had a forward facing rounded head with an area of $7.45 \mathrm{~mm}^{2}$ (Fig $5.3 \mathrm{c}$ ) and serrated mandibles (Fig $5.3 \mathrm{~d}-\mathrm{g}$ ). Behavioural Trials (Fig $5.3 \mathrm{~h}$ ): Larvae of the Angel rest on the trunk of the tree during the day and move to the leaves at night to feed (Wagner 2005). Caterpillars were resting on the dorsal or ventral side of the leaf or on the individual sprig containers prior to experimentation. When attacked posteriorly the caterpillars would thrash their head towards the site of attack but also flick their posterior end away from the attack, with continued attacks the caterpillar dropped from the leaf. Anterior attacks caused the larvae 
to thrash away from the pinch and curl-in and in one case drop from the leaf. Once on the ground, 2/3 caterpillars crawled away. No sound production was observed. It has previously been reported that, when alarmed, the caterpillar raises its second thoracic segment to reveal its patch of red hair (Wagner 2005). I did observe the caterpillar revealing the patch, but not during attack trials. I suggest that the hairs of this species act as its main defence. Hairs have been shown to be effective against ants and bugs (Dyer 1997) and chickadees did not attempt to attack hairy caterpillars when given the opportunity, but when they were hungry and given no alternatives they did consume the insides of some species (Heinrich and Collins 1983). 


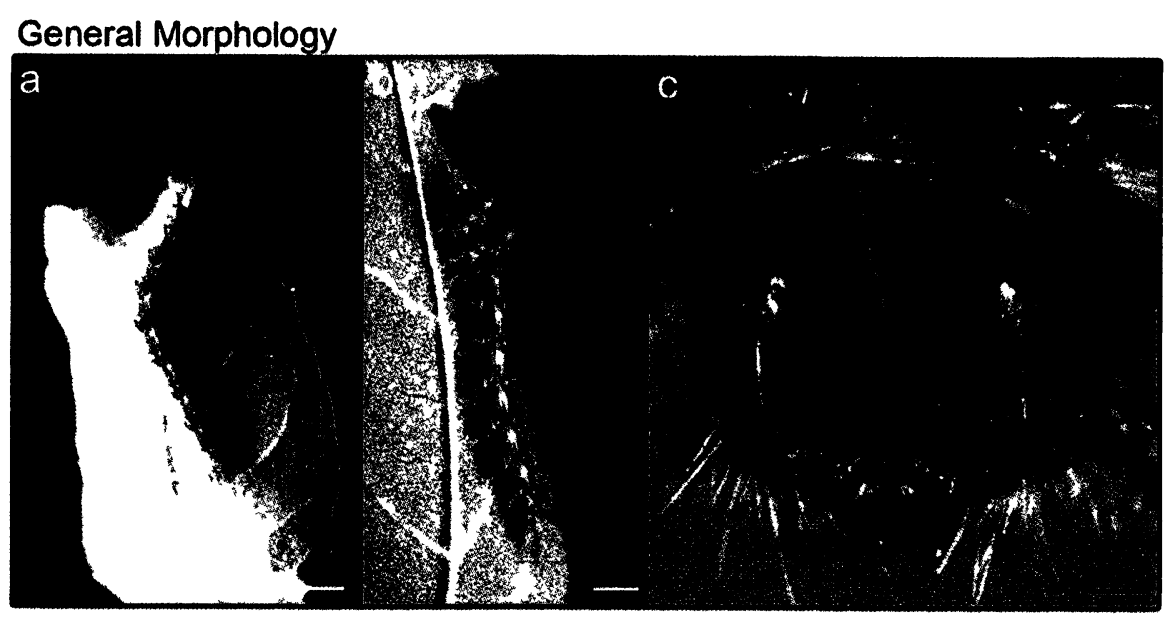

Mandibles

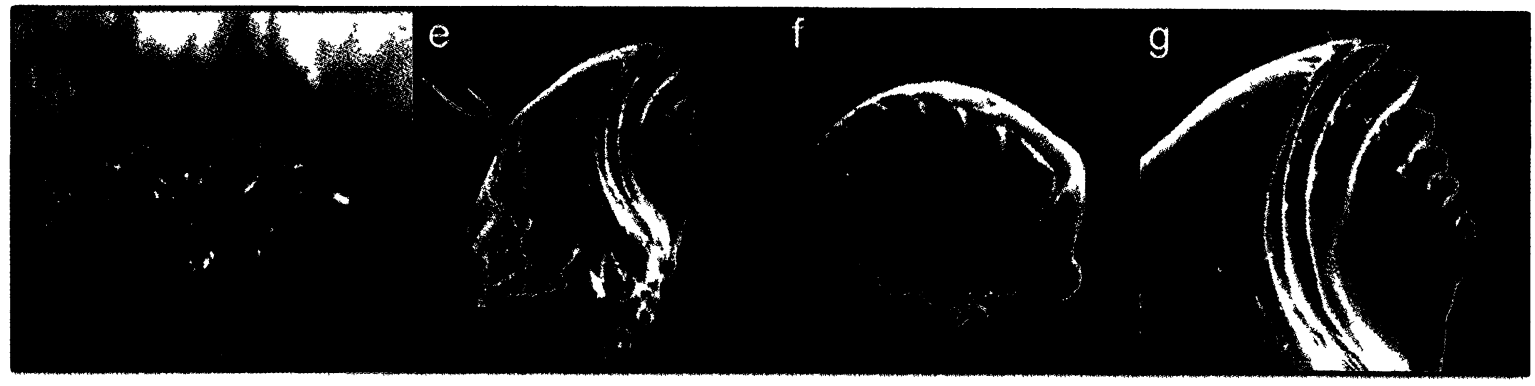

\section{Defensive Behaviours}

\begin{tabular}{|ll|}
\hline h Sound Production & $\square$ Biting \\
$\square$ Regurgitation & $\square$ Curling-in \\
$\square$ Chemical defense & $\square$ Curling up \\
$\square$ Thrashing & $\square$ Twitching \\
$\square$ Major thrashing & $\square$ Walk away \\
$\square$ Directed thrashing & $\square$ Dropping \\
\hline
\end{tabular}

Figure 5.3 Olceclostera angelica, the Angel. General morphological features of a late instar caterpillar. (a) Dorsal view of a late instar on its hostplant, scale bar $0.75 \mathrm{~cm}$. (b) Lateral view of a fifth instar caterpillar, scale bar $0.5 \mathrm{~cm}$. (c) Dorso-anterior view of the head capsule of a fifth instar larva, scale bar $0.5 \mathrm{~mm}$. Mandibles of a late instar caterpillar. (d) The mandibles of a fifth instar in closed position, scale bar $0.5 \mathrm{~mm}$; (e) right mandible, ventro-anterior view, scale bar $0.25 \mathrm{~mm}$; (f) left mandible, inner face, scale bar $0.25 \mathrm{~mm}$; (g) close up of anterior edge of right mandible, scale bar $0.1 \mathrm{~mm}$. (h) List of defensive behaviours observed upon attack and when handled. All images taken by Veronica Bura. 


\section{FAMILY: SATURNIIDAE}

This family encompasses just under 1900 species from eight subfamilies, Ceratocampinae, Hemileucinae, Saturniinae, Salassinae, Asenurinae, Cercophaninae, Oxyteninae and Agiliinae. Results are presented here for 6 species from Ceratocampinae, 3 from Hemileucinae and 6 Saturniinae. Larvae of this family, with few exceptaions, bear scoli or tubercles on their bodies (Tuskes et al. 1996).

\section{SUBFAMILY: CERATOCAMPINAE}

The scoli of this subfamily are long horn-like tubercles (Tuskes et al. 1996) that are not derived from secretory scoli (Deml and Dettner 2002).

Anisota virginiensis (Drury), the Pink-Striped Oakworm, Fig 5.4

The Pink-Striped Oakworm is a new world moth found throughout eastern North America (Tuskes et al. 1996, Wagner 2005). As their name implies, caterpillars of this species feed on oak (Quercus sp.) a member of the Fagaceae (Tuskes et al. 1996, Wagner 2005).

Morphology: Late instar $A$. virginiensis are pink and black striped, have a number of black projections covering their body, the longest of which are on the second thoracic segment and an orange-red head (Fig $5.4 \mathrm{a}, \mathrm{b}$ ). Fifth instar larvae were, on average, $4 \mathrm{~cm}$ in length $(\mathrm{n}=5)$, had an oval head with an area of $12.17 \mathrm{~mm}^{2}$ (Fig $\left.5.4 \mathrm{c}\right)$ and smooth mandibles (Fig $5.4 \mathrm{~d}$-g).

Behavioural Trials (Fig 5.4 h): Prior to experimentation the caterpillars were resting along the mid-vein on the underside of the leaf of hostplant. Both anterior and posterior attacks elicited no observable reaction in these caterpillars, however, in one trial the larva was eating and the attack caused it to stop eating. No sound production was observed. 

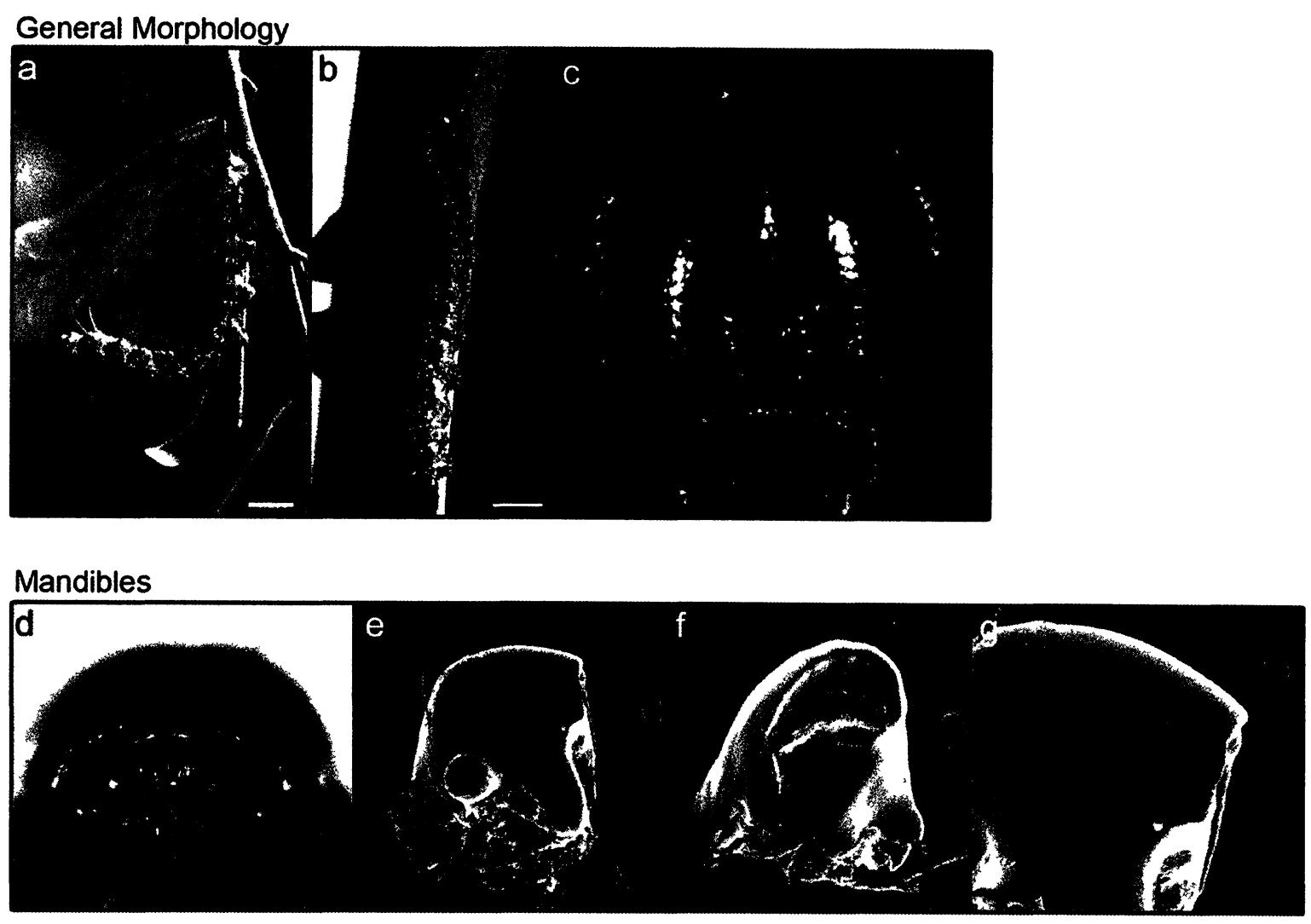

\section{Defensive Behaviours}

\begin{tabular}{|ll|}
\hline $\mathrm{h} \square$ Sound Production & $\square$ Biting \\
$\square$ Regurgitation & $\square$ Curling-in \\
$\square$ Chemical defense & $\square$ Curling up \\
$\square$ Thrashing & $\square$ Twitching \\
$\square$ Major thrashing & $\square$ Walk away \\
$\square$ Directed thrashing & $\square$ Dropping \\
\hline
\end{tabular}

Figure 5.4 Anisota virginiensis, the Pink-Striped Oakworm. General morphological features of a late instar caterpillar. (a) Lateral view of a late instar larva on its hostplant, scale bar $0.5 \mathrm{~cm}$. (b) Lateral view of a late instar caterpillar, scale bar $0.5 \mathrm{~cm}$. (c) Anterior view of the head capsule of a fifth instar caterpillar, scale bar $0.5 \mathrm{~mm}$. Mandibles of a late instar caterpillar. (d) The mandibles of a fifth instar larva in closed position, scale bar $0.5 \mathrm{~mm}$; (e) right mandible, ventroanterior view, scale bar $0.25 \mathrm{~mm}$; (f) left mandible, inner face, scale bar $0.25 \mathrm{~mm}$; (g) close up of the anterior ridge of the right mandible, scale bar $0.1 \mathrm{~mm}$. (h) List of defensive behaviours observed upon attack and when handled. All images taken by Veronica Bura, except (a) by Troy Bartlett (http://bugguide.net/node/view/1855/bgimage). 
Citheronia lobesis Rothschild, Fig 5.5

Citheronia lobesis is a new world moth found in Mexico and Central American and belongs to the subfamily Ceratocampinae (Janzen and Hallwachs 2009, Savela 2009). Caterpillars of this species feed on plants from ten different families, including Anacardiaceae, Rubiaceae and Viscaceae (Janzen and Hallwachs 2009).

Morphology: Late instar caterpillars of $C$. lobesis are a cream-brown colour with a black ventral side and many scolus-projections, especially at the anterior end (Fig $5.5 \mathrm{a}, \mathrm{b}$ ). At the time of experimentation the caterpillars were in their penultimate instar, $5.15 \mathrm{~cm}$ in length $(\mathrm{n}=2)$, had a rounded head with an area of $10.18 \mathrm{~mm}^{2}$ (Fig $\left.5.5 \mathrm{c}\right)$ and serrated mandibles (Fig $5.5 \mathrm{~d}-\mathrm{g}$ ).

Behavioural Trials: These large caterpillars are very visible on at least one of their foodplants (Cochlospermum vitifolium) as there are very few leaves and the larva rests on the bare stems and petioles (Miller et al. 2006). The two specimens I tested were found on the side of a road also resting out in the open on the branch of a tree (Fig 5.5 a). Prior to experimentation the larvae were resting on the stem of their hosplant sprig. Anterior attacks caused the larva to thrash away from the pinch and stridulate (see below). The caterpillar walked away from the immediate area after subsequent pinches. When attacked posteriorly caterpillars responded by thrashing towards the pinch, stridulating and regurgitating a lot. Again one larva left the immediate area following subsequent attacks. In both types of attacks the caterpillars also fanned out their thoracic scoli. Acoustics: Caterpillars of $C$. lobesis stridulated in response to attack.

Sound characteristics (Table 5.3): The acoustic signals produced by C. lobesis consist of short trains of stridulations, or chirps (Fig 5.6 a,b). Trains were on average $304.85 \pm$ 
$232.79 \mathrm{~ms}$ in duration and contained $1-3$ chirps ( $\mathrm{n}=4$ trains from 1 animal). Chirps were on average $109.99 \pm 42.19 \mathrm{~ms}$ in duration and contained 6-32 components ( $\mathrm{n}=7$ chirps). Sounds were broadband (mean $\mathrm{Q} 3=4.65 \pm 3.80, \mathrm{Q} 10=0.64 \pm 0.14, \mathrm{n}=5$ chirps from 1 animal) and had a mean dominant frequency of $32.10 \pm 10.76 \mathrm{kHz}$ (Fig $5.6 \mathrm{c}$ ). Mechanism: I suspect that chirps are produced using the mandibles. The anterior edge of one mandible slides against the inner face of the other to create chirps. The friction between the two parts is what creates the sound, much like rubbing two marbles together. The 'track marks' on the inner faces of the mandibles lend evidence to this suspicion (Fig $5.5 \mathrm{f}, \mathrm{g})$. These track marks are also seen on the mandibles of another sound producer ( $S$. pyri, see below) but are not present on the serrated mandibles of a non-sound producer (O. angelica). Video analysis should be performed to confirm this.

Behaviours associated with sound production and proposed function: Though the caterpillar did produce both sound and regurgitant, the behaviours did not seem as closely associated as they have in other species (see below). In the first five pinches of the attack trials one of the two caterpillars produced both sound and regurgitation (Fig $5.5 \mathrm{i}$ ). In this instance the sound preceded regurgitation. The larvae did, however, fan out their thoracic scoli. This behaviour was also been observed in urticating species (see below) and I believe is a way of increasing the surface area of their chemical defence. The scoli of $C$. lobesis are not urticating (Miller et al. 2006) so perhaps this behaviour is part of a bluff or startle display. Sound production occurred as the caterpillar would thrash towards the site of attack (in posterior attacks) as would the fanning out of the spines. The possibility that the stridulations are warning of impending regurgitation still remains, however, these caterpillars are palatable to at least some bird species as they are known to be fed to 
nestlings of the Black-headed Trogon (Trogon melanocephalus) (Miller et al. 2006). Further tests to tease apart these two possibilities are still required. 


\section{General Morphology}

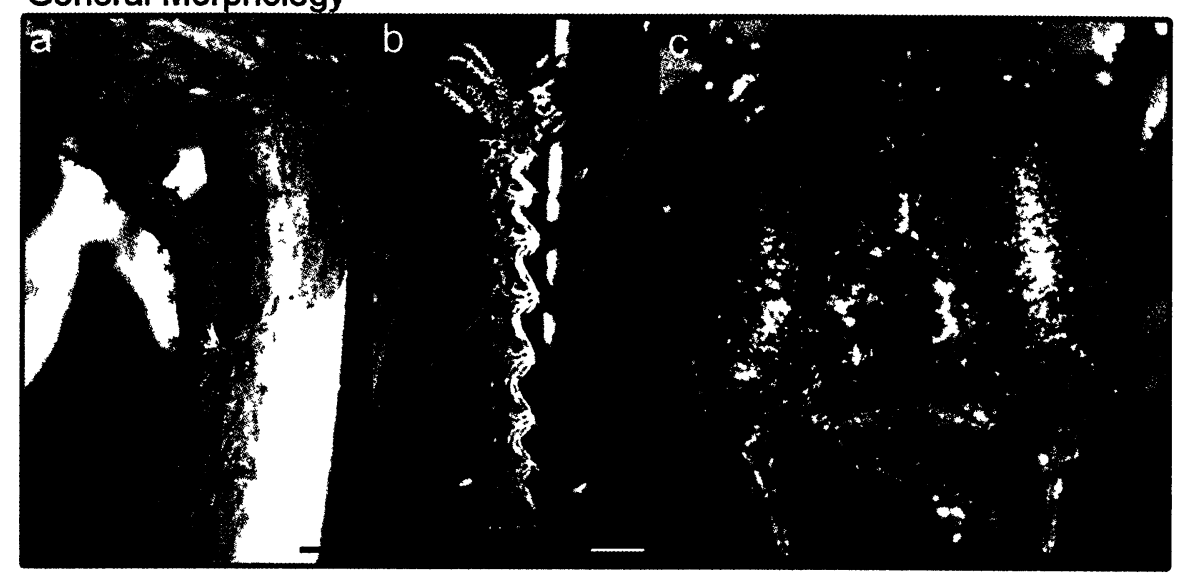

Mandibles

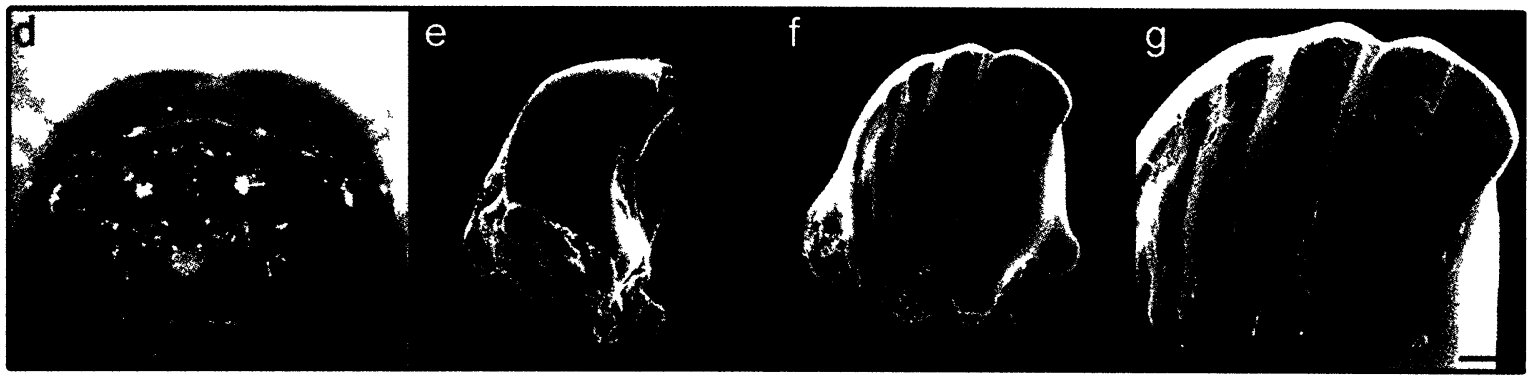

Defensive Behaviours

\begin{tabular}{|ll|}
\hline $\mathrm{h} \square$ Sound Production & $\square$ Biting \\
$\square$ Regurgitation & $\square$ Curling-in \\
$\square$ Chemical defense & $\square$ Curling up \\
$\square$ Thrashing & $\square$ Twitching \\
$\square$ Major thrashing & $\square$ Walk away \\
$\square$ Directed thrashing & $\square$ Dropping \\
\hline
\end{tabular}
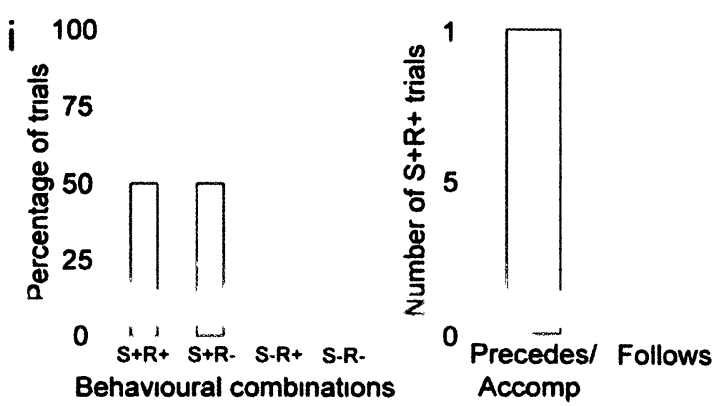

Figure 5.5 Citheronia lobesis. General features of a late instar caterpillar. (a) Lateral view of a penultimate instar on its hostplant, scale bar $1 \mathrm{~cm}$. (b) Lateral view of a final instar caterpillar, scale bar $1 \mathrm{~cm}$. (c) Anterior view of the head capsule of a penultimate instar, scale bar $0.5 \mathrm{~mm}$. Mandibles of a late instar caterpillar. (d) The mandibles of a penultimate instar in closed position, scale bar $0.5 \mathrm{~mm}$; (e) right mandible, ventro-anterior view, scale bar $0.25 \mathrm{~mm}$; (e) left mandible, inner face, scale bar $0.25 \mathrm{~mm}$; (g) close up of anterior edge of left mandible, scale bar $0.1 \mathrm{~mm}$. (h) List of defensive behaviours observed upon attack and when handled. (i) Histograms showing the relationship between sound production (S) and regurgitation (R) during the first 5 pinches of an attack $(n=2)$. All images taken by Veronica Bura, except (a) by Alan Fleming and (b) by Dan Janzen. 
a

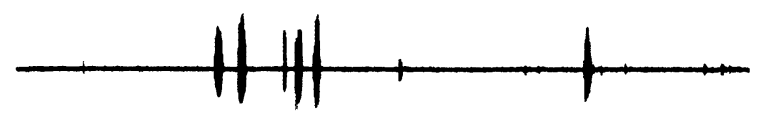

b
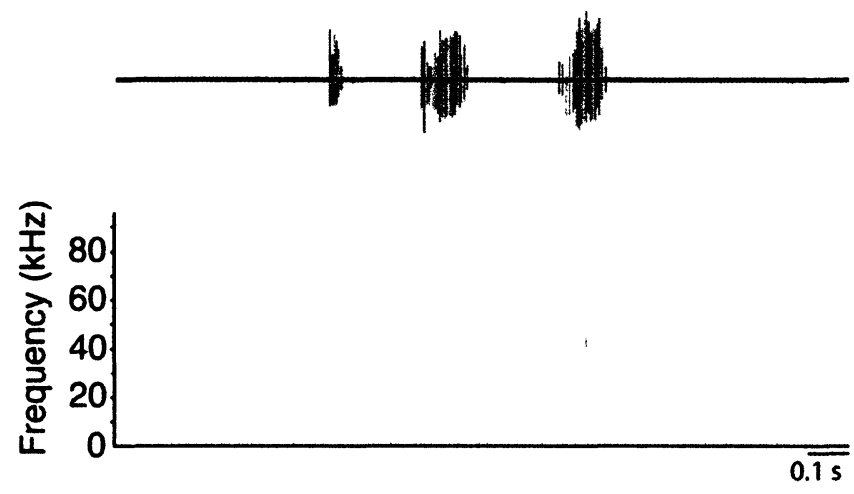

C

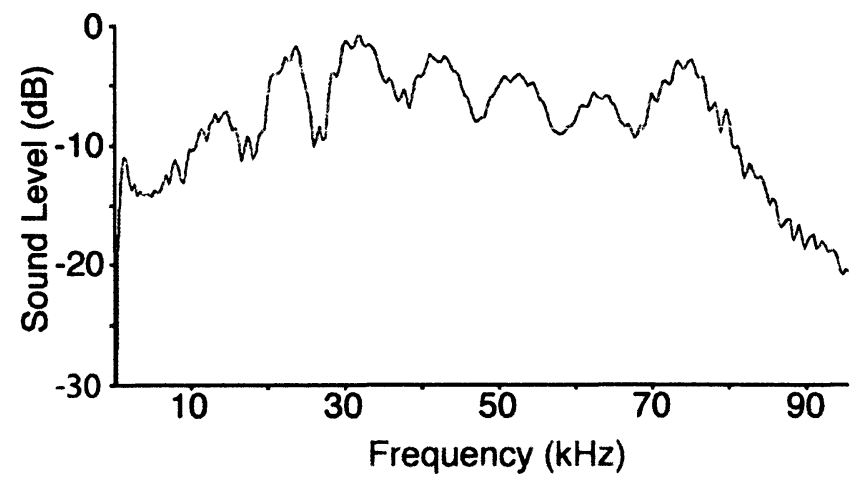

Figure 5.6 Sounds produced by late instar $\boldsymbol{C}$. lobesis. (a) Oscillogram of chirps following attack. (b) Time expansion of bracket in (a) showing the multiple components of each chirp. The accompanying spectrogram shows the frequency distribution of each chirp. (c) Power spectrum taken from one individual. 
Dryocampa rubicunda (Fabricius), the Rosy Maple moth, Fig 5.7

The Rosy Maple moth is a new world moth found throughout eastern North America (Tuskes et al. 1996, Wagner 2005). Caterpillars of this species feed principally of maple (Acer sp.), though there have been some reported on oak (Quercus sp.) (Tuskes et al. 1996, Wagner 2005).

Morphology: Late instar caterpillars of $D$. rubicunda are yellow to pale-green with longitudinal stripes, a red head and black projections on its body, the longest of which are on the second thoracic segment (Fig $5.7 \mathrm{a}, \mathrm{b}$ ). Fifth instar larvae were approximately $4 \mathrm{~cm}$ in length, on average $(\mathrm{n}=5)$, had an oval head with an area of $14.38 \mathrm{~mm}^{2}$ (Fig $\left.5.7 \mathrm{c}\right)$ and smooth mandibles (Fig $5.7 \mathrm{~d}-\mathrm{g}$ ).

Behavioural Trials (Fig 5.7 h): Prior to experimental trials, the caterpillars could be found on either the dorsal or ventral side of the leaf, feeding at the edge. When attacked posteriorly the caterpillars responded by thrashing towards the site of attack and regurgitating. Anterior attacks caused the larvae to thrash away from the pinch and regurgitate. No sound production was observed. Caterpillars from this species were not attacked by chickadees, even when they walked in front of the birds and were classified as unpalatable (Heinrich and Collins 1983). 


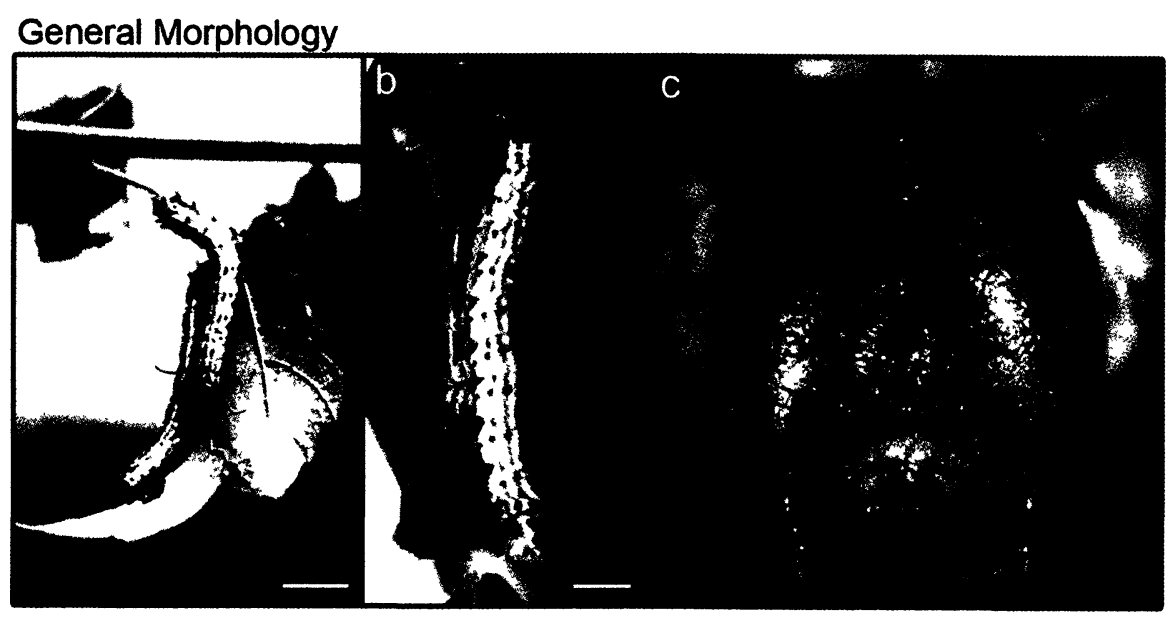

Mandibles

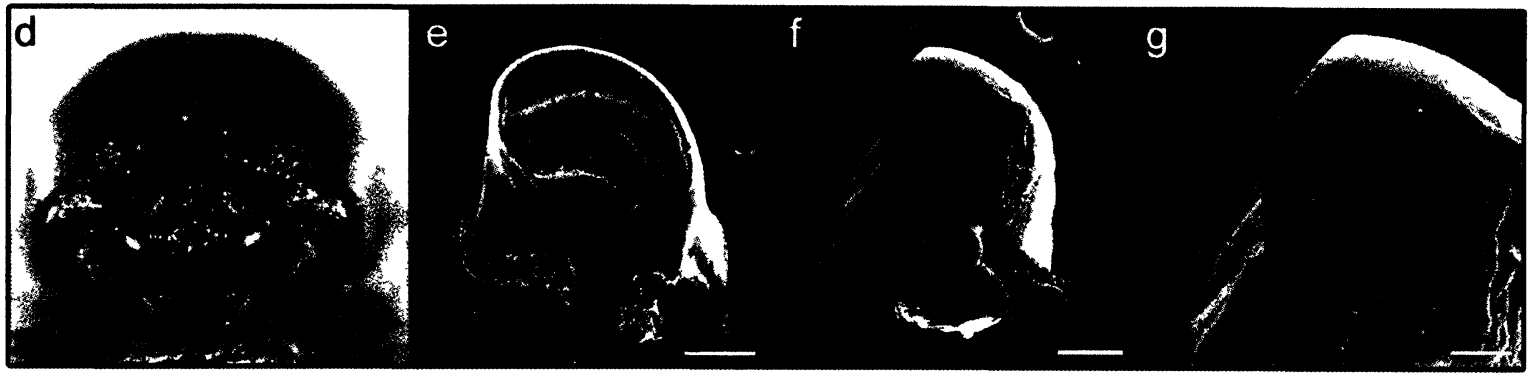

Defensive Behaviours

\begin{tabular}{|ll|}
\hline h Sound Production & $\square$ Biting \\
$\square$ Regurgitation & $\square$ Curling-in \\
$\square$ Chemical defense & $\square$ Curling up \\
$\square$ Thrashing & $\square$ Twitching \\
$\square$ Major thrashing & $\square$ Walk away \\
$\square$ Directed thrasning & $\square$ Dropping \\
\hline
\end{tabular}

Figure 5.7 Dryocampa rubicunda, the Rosy Maple moth. General morphological features of a late instar caterpillar. (a) Lateral view of two caterpillars on their hostplant, scale bar $1 \mathrm{~cm}$. (b) Lateral view of a fifth instar larva, scale bar $0.5 \mathrm{~cm}$. (c) Anterior view of the head capsule of a fifth instar larva, scale bar $0.5 \mathrm{~mm}$. Mandibles of a late instar caterpillar. (d) The mandibles of a fifth instar caterpillar in closed position, scale bar $0.5 \mathrm{~mm}$; (e) right mandible, inner face, scale bar $0.25 \mathrm{~mm}$; (f) left mandible, ventro-anterior view, scale bar $0.25 \mathrm{~mm}$; (g) close up of the anterior edge of the left mandible, scale bar $0.125 \mathrm{~mm}$. (h) List of defensive behaviours observed upon attack and when handled. All images taken by Veronica Bura, except (a) by Terry Price (http://www.forestryimages.org/images/768x512/1247211.jpg). 


\section{Eacles imperialis (Drury), the Imperial moth, Fig 5.8}

The Imperial moth is a new world moth found throughout eastern North America (Tuskes et al. 1996, Wagner 2005). According to Tuskes et al. (1996) the E. Imperialis in the Ottawa region would be considered E. Imperialis pini, a subspecies, but this separation is not indicated in other sources (e.g. Wagner 2005). Caterpillars of the Imperial moth feed on members from 10 families of plants, both coniferous and deciduous, including Pinus sp., Picea sp., Betula sp. and Quercus sp. (Tuskes et al. 1996, Wagner 2005).

Morphology: Caterpillars of $E$. Imperialis can occur in a number of different morphs, green, brown-black, red, orange, or a combination of these (Tuskes et al. 1996, Wagner 2005). The brood that I reared originally contained both black and green morphs, but they had all turned black or black-green by their final instar (Fig 5.8 a,b). These caterpillars had large white spiracles, white and black scoli projecting from their body, were covered in fine white hair and had a yellow and black head. Caterpillars were extremely difficult to spot on their hostplant. Fifth instar caterpillars were about $6.7 \mathrm{~cm}$ in length at the time of experimentation, had rounded heads with an area of $28.74 \mathrm{~mm}^{2}$ (Fig 5.8c) and serrated mandibles (Fig $5.8 \mathrm{~d}-\mathrm{g}$ ).

Behavioural Trials (Fig $5.8 \mathrm{~h}$ ): Prior to experimentation the caterpillar was resting on a sprig of hostplant. When attacked posteriorly the caterpillars responded first by regurgitating then thrashing towards the site of attack and subsequently from side to side. Anterior attacks caused the larvae to curl-in and thrash away from the pinch, and then regurgitate and thrash slightly from side to side if further harassed. No sound production was observed. I suggest that crypsis is the main defence in this species. 
General Morphology

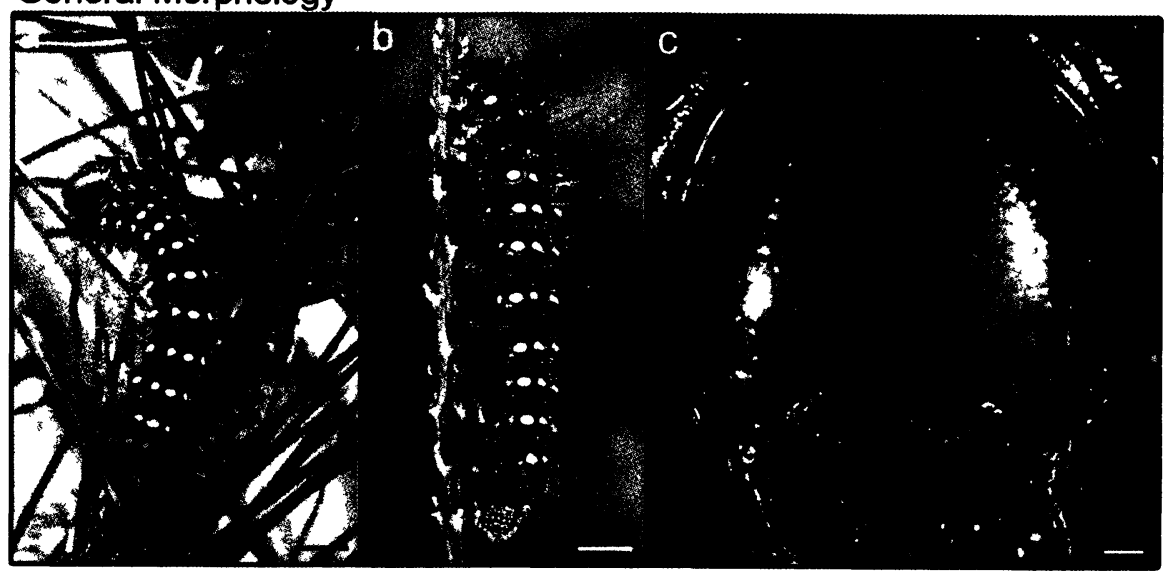

Mandibles

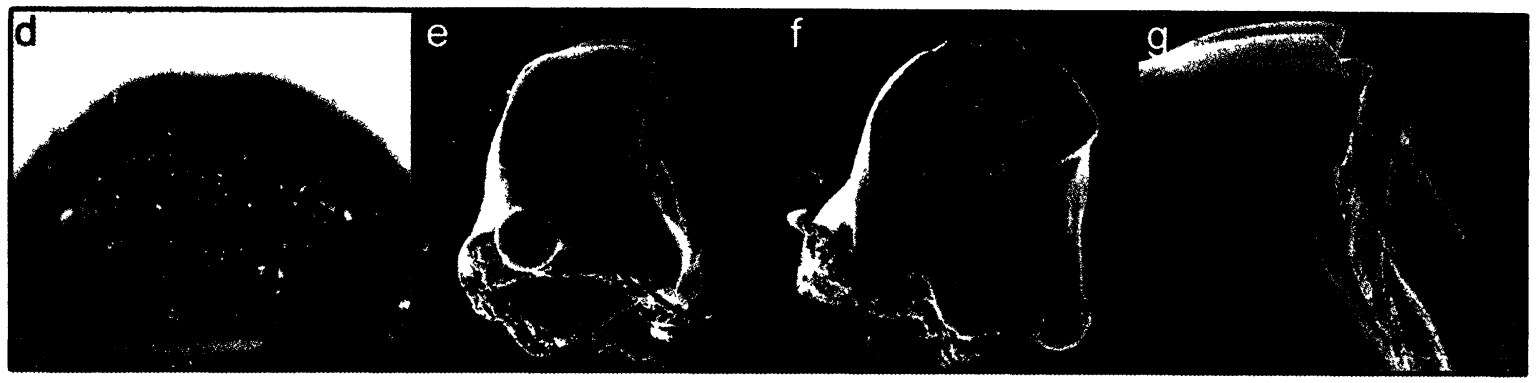

\section{Defensive Behaviours}

\begin{tabular}{|ll|}
\hline$\square$ Sound Production & $\square$ Biting \\
$\square$ Regurgitation & $\square$ Curling-in \\
$\square$ Chemical defense & $\square$ Curling up \\
$\square$ Thrashing & $\square$ Twitching \\
$\square$ Major thrashing & $\square$ Walk away \\
$\square$ Directed thrashing & $\square$ Dropping \\
\hline
\end{tabular}

Figure 5.8 Eacles imperialis, the Imperial moth. General morphological features of a late instar caterpillar. (a) Lateral view of a late instar on its hostplant, scale bar $1 \mathrm{~cm}$. (b) Lateral view of a fifth instar caterpillar, scale bar $0.75 \mathrm{~cm}$. (c) Anterior view of the head capsule of a fifth instar, scale bar $0.5 \mathrm{~mm}$. Mandibles of a late instar caterpillar. (d) The mandibles of a fifth instar in closed position, scale bar $0.5 \mathrm{~mm}$; (e) right mandible, ventro-anterior view, scale bar $0.25 \mathrm{~mm}$; (f) left mandible, inner face, scale bar $0.25 \mathrm{~mm}$; (g) close up of anterior edge of right mandible, scale bar $0.1 \mathrm{~mm}$. (h) List of defensive behaviours observed upon attack and when handled. All images taken by Veronica Bura, except (b) by Jayne Yack. 
Eacles ormondei Schaus, Fig 5.9

Eacles ormondei is a new world moth found from Mexico, through Central America to Ecuador (Savela 2009). Caterpillars eat plant species from 8 different families, including Malvaceae, Rosaceae and Anacardiaceae (Janzen and Hallwachs 2009).

Morphology: Eacles ormondei can appear in a number of morphs, including green, yellow and orange (Janzen and Hallwachs 2009). The late instar caterpillar was orange with a red-purple stripe along its lateral sides and covered in non-urticating scolus-like projections that were longer at the anterior end (Fig $5.9 \mathrm{a}, \mathrm{b})$. The specimen I tested was approximately $7 \mathrm{~cm}$ in length at the time of experimentation.

Behavioural Trials (Fig 5.9 c): The caterpillar was feeding from the underside of leaves of its hostplant prior to the trial. Posterior attacks caused the larva to thrash towards the site of attack, return to a straight position and twitch. After a couple of pinches as the caterpillar thrashed towards the forceps it would regurgitate. This could be observed both visually and audibly since there was a bubbling sound that accompanied the production of regurgitant (dicussed in part B). When attacked anteriorly the caterpillar thrashed away from the pinch and then continued from side to side, though this movement was not very elaborate. No sound production was observed. 
General Morphology

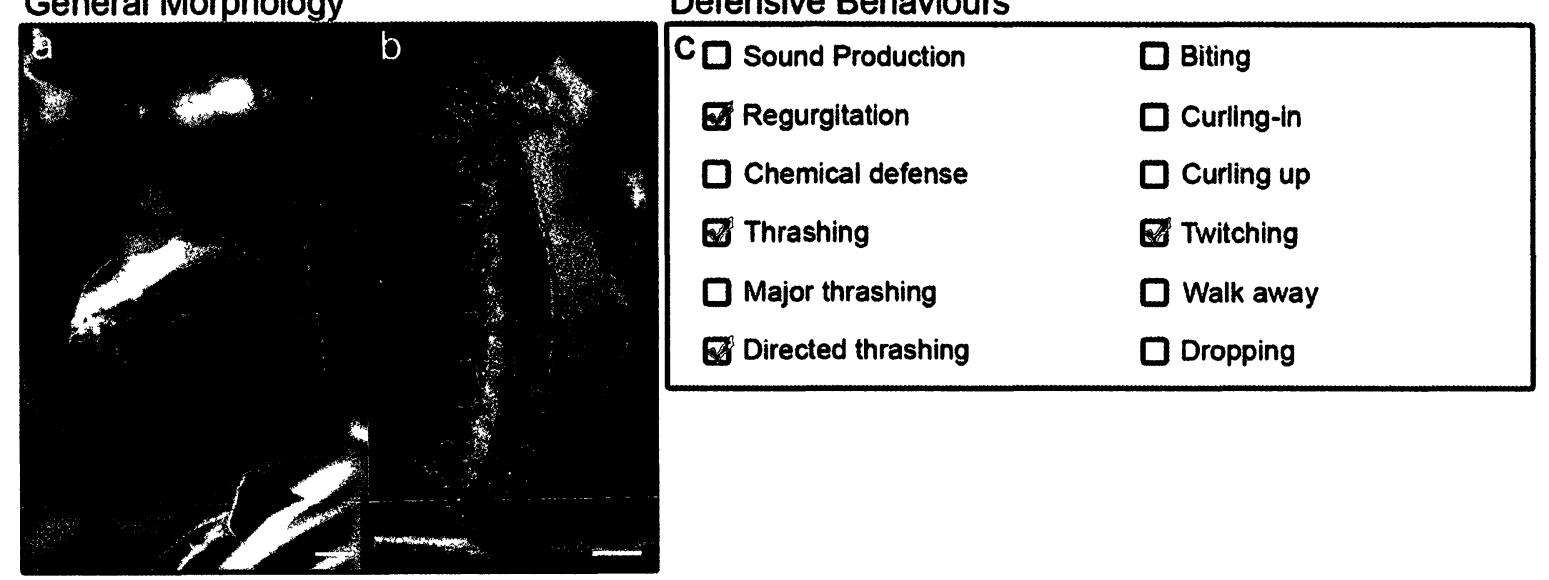

Figure 5.9 Eacles ormondei. General morphological features of a late instar caterpillar. (a) Lateral view of a late instar caterpillar on its hostplant, scale bar $1 \mathrm{~cm}$. (b) Lateral view of a late instar caterpillar, scale bar $0.75 \mathrm{~cm}$. (c) List of defensive behaviours observed upon attack and when handled. Image in (a) by Jayne Yack and (b) by Alan Fleming. 
Schausiella santarosensis Lemaire, Fig 5.10

Schausiella santarosensis is a new world moth endemic to Costa Rica (Miller et al. 2006, Janzen and Hallwachs 2009). Caterpillars of this species feed on mature leaves in the crowns of Hymenaea courbaril, a tall tree from the family Fabaceae (Miller et al. 2006, Janzen and Hallwachs 2009).

Morphology: Final instar caterpillars of $S$. santarosensis are large and green with four dorsal scolus-projections on their thoracic segments and their bodies are covered in little pink-red tubercles (Fig $5.10 \mathrm{a}, \mathrm{b}$ ). The ultimate instar caterpillar was $6.7 \mathrm{~cm}$ in length at the time of experimentation, had a rounded head with an area of $34.04 \mathrm{~mm}^{2}$ (Fig $5.10 \mathrm{c}$ ) and smooth mandibles (Fig $5.10 \mathrm{~d}-\mathrm{g}$ ).

Behavioural Trials (Fig $5.10 \mathrm{~h}$ ): I tested one final instar and two penultimate instars of this species. Prior to experimentation the caterpillars were resting on the stem of their hostplant. When attacked posteriorly the caterpillars responded by thrashing towards the site of attack, while stridulating, followed by a short burst of side to side thrashing, then returning to a straight position and twitching. Anterior attacks resulted in thrashing away from the pinch, and then side to side in a wide arc. In both sets of attacks regurgitation also occurred with increased attacks.

Acoustics: Schausiella santarosensis caterpillars produce bursts of stridulation (chirps) in response to attack.

Sound characteristics (Table 5.3): Chirps were produced in bursts of one to three instead of longer trains as seen in other species (e.g. S. pyri, see below) and were, on average, $193.66 \pm 245.96 \mathrm{~ms}$ in duration (Fig $5.11 \mathrm{a}, \mathrm{b} ; \mathrm{n}=3$ trains from 1 animal). Chirps were $57.35 \pm 15.29 \mathrm{~ms}$ in duration and contained $6-10$ components $(\mathrm{n}=4$ chirps). Sounds were 
broadband $(\mathrm{Q} 3=4.38 \pm 2.22, \mathrm{Q} 10=0.71 \pm 0.40, \mathrm{n}=5$ chirps from 1 animal $)$ and had $\mathrm{a}$ mean dominant frequency of $38.25 \pm 1.59 \mathrm{kHz}$ (Fig $5.11 \mathrm{c})$.

Mechanism: Though the mechanism of sound production was not investigated, I assume that sounds are produced using the mandibles, as in the other stridulating species. Further examination is required.

Behaviours associated with sound production and proposed function: Chirps were produced as the caterpillar thrashed; one chirp per body flick. Regurgitation followed the appearance of sound but later in the trial. These chirps may serve as a warning for the impending regurgitation, as in other species, though the association is not as strong, as during the first five pinches of the attack, no regurgitation was noted (Fig $5.10 \mathrm{i}$ ). The sounds were tightly associated with thrashing movements, and so may act as a signal of unprofitability, that the predator should move on because it will be time consuming to secure this caterpillar. 


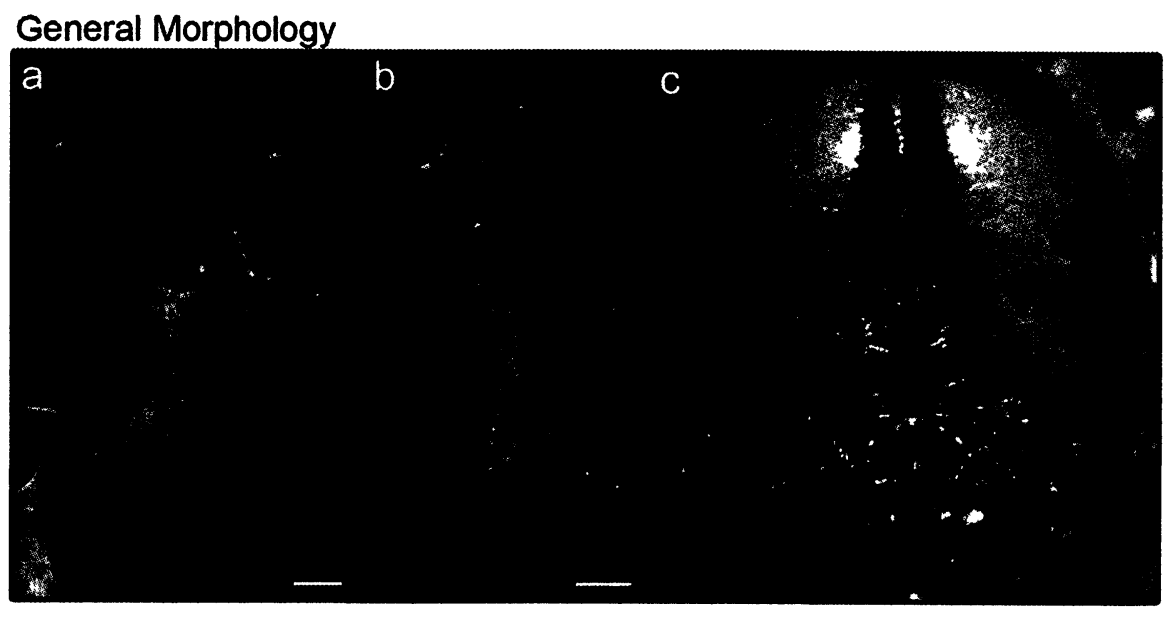

\section{Mandibles}

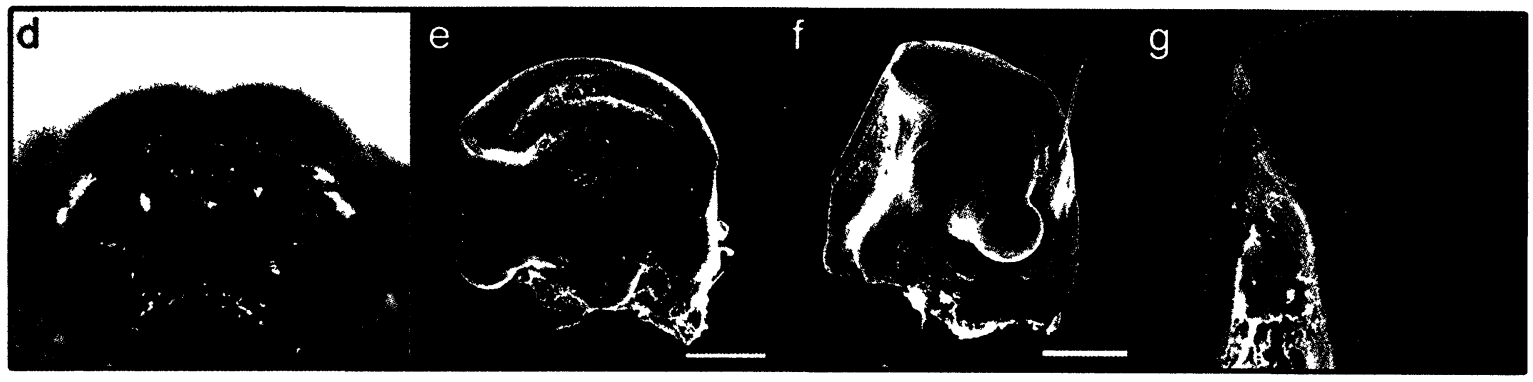

\section{Defensive Behaviours}

\begin{tabular}{|ll|}
\hline $\mathrm{W}$ Sound Production & $\square$ Biting \\
$\square$ Regurgitation & $\square$ Curling-in \\
$\square$ Chemıcal defense & $\square$ Curling up \\
$\square$ Thrashing & $\square$ Twitching \\
$\square$ Major thrashing & $\square$ Walk away \\
$\square$ Directed thrashing & $\square$ Dropping \\
\hline
\end{tabular}

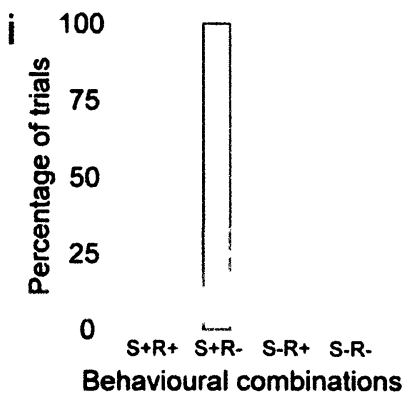

Figure 5.10 Schausiella santarosensis. General morphological characteristics of a late instar caterpillar. (a) Lateral view of a late instar on its hostplant, scale bar $1 \mathrm{~cm}$. (b) Lateral view of a final instar caterpillar, scale bar $0.75 \mathrm{~cm}$. (c) Anterior view of the head capsule of a final instar caterpillar, scale bar $0.5 \mathrm{~mm}$. Mandibles of a late instar caterpillar. (d) The mandibles of a final instar larva in closed position, scale bar $0.5 \mathrm{~mm}$; (e) right mandible, inner face, scale bar $0.5 \mathrm{~mm}$; (f) left mandible, ventro-anterior view, scale bar $0.5 \mathrm{~mm}$; (g) close up of anterior edge of left mandible, scale bar $0.1 \mathrm{~mm}$. (h) List of defensive behaviours observed upon attack and when handled. (i) Histogram showing the relationship between sound production (S) and regurgitation (R) during the first 5 pinches of an attack $(n=2)$. All images taken by Veronica Bura, except (a) by Dan Janzen and (b) by Alan Fleming. 
a

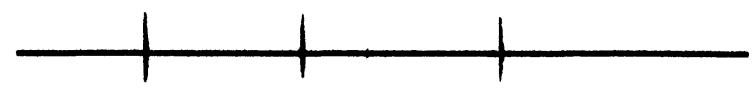

b
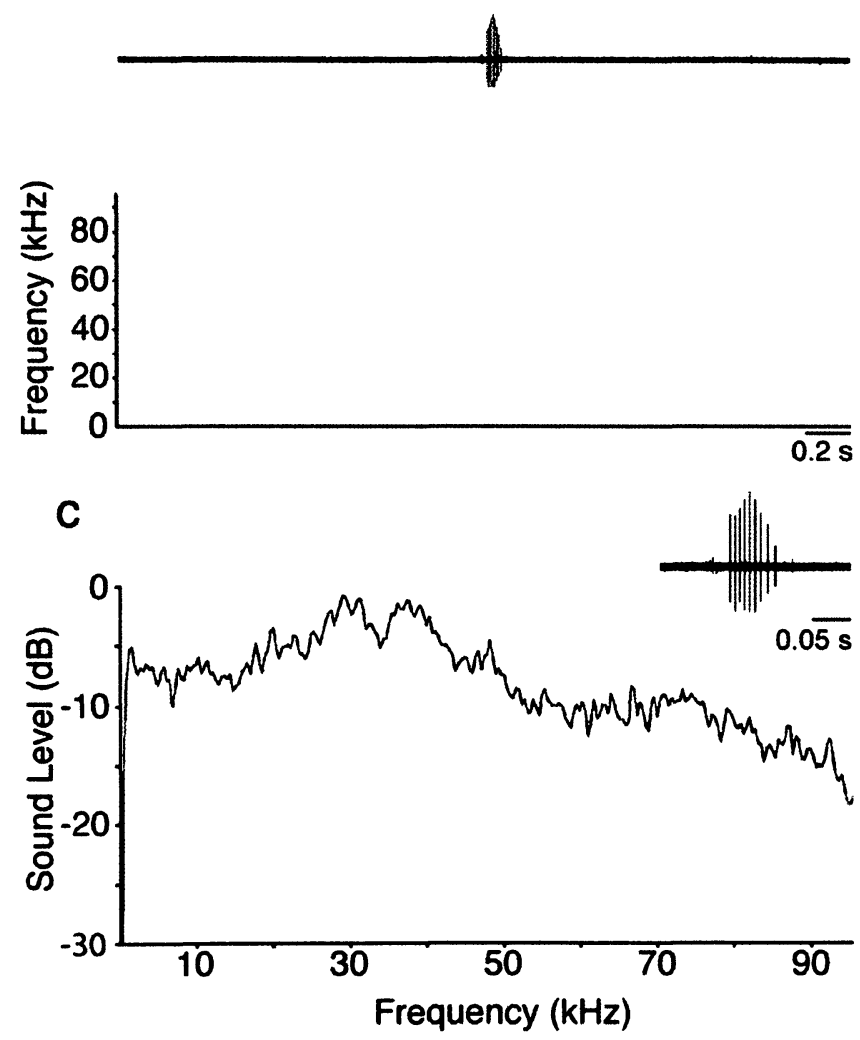

Figure 5.11 Sounds produced by late instar $S$. santarosensis. (a) Oscillogram of chirps following attack. (b) Time expansion of chirp indicated by black dot in (a). The accompanying spectrogram shows the frequency distribution. (c) Power spectrum taken from one individual. Further expanded time scale of chirp in (b) is inset showing the multiple components. 


\section{SUBFAMILY: HEMILEUCINAE}

Caterpillars of this subfamily have the shared characteristic of urticating scoli that deliver their chemicals by injection (Deml and Dettner 2002).

Automeris postalbida Schaus, Fig 5.12

Automeris postalbida is a new world moth found from Costa Rica to Ecuador (Savela 2009). The specimen that I tested has been identified as A. postalbidaDHJ07 and feeds on species from 25 different plant families (Janzen and Hallwachs 2009). Morphology: Caterpillars of $A$. postalbida are a rusty-orange colour with a pale stripe running the length of its lateral sides, and a dark patch on their dorsal posterior ends between long pink scoli (Fig $5.12 \mathrm{a}, \mathrm{b}$ ). It is covered in multi-branched urticating scoli that are more concentrated at the anterior and posterior ends. The specimen I tested was approximately $10 \mathrm{~cm}$ in length.

Behavioural Trials (Fig 5.12 c): The caterpillar was resting on a barren portion of its hostplant prior to experimentation. When attacked posteriorly the caterpillar fanned out its scoli, presumably to cover more area with its chemical defence, and thrashed towards the site of attack after multiple pinches. The fanned out scoli revealed a large black patch on the dorsal surface of the $8^{\text {th }}$ abdominal segment. When attacks were directed at the prolegs the directed thrashing was more evident. Anterior attacks caused the caterpillar to curl-in (scoli continued to be fanned out). No sound production was observed. I suspect the main defence in this species is its urticating spines. 
General Morphology

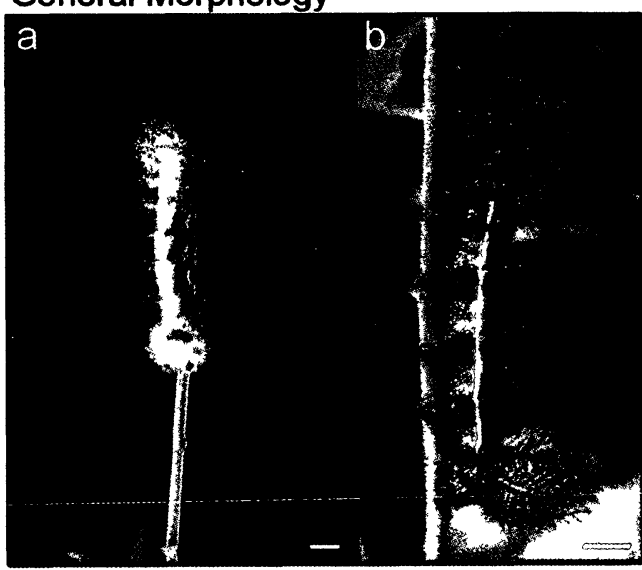

Defensive Behaviours

\begin{tabular}{|ll|}
\hline$\square$ Sound Production & $\square$ Biting \\
$\square$ Regurgitation & Curling-in \\
$\square$ Chemical defense & $\square$ Curling up \\
$\square$ Thrashing & $\square$ Twitching \\
$\square$ Major thrashing & $\square$ Walk away \\
$\square$ Directed thrashing & $\square$ Dropping \\
\hline
\end{tabular}

Figure 5.12 Automeris postalbida. General morphological features of a late instar caterpillar. (a) Dorsal view of a final instar caterpillar on a twig of hostplant, scale bar $1 \mathrm{~cm}$. (b) Lateral view of a fifth instar larva, scale bar $1 \mathrm{~cm}$. (c) List of defensive behaviours observed upon attack and when handled. Image in (a) by Jayne Yack and (b) by Alan Fleming. 
Automeris io (Fabricius), the Io moth, Fig 5.13

The Io moth is a new world moth found throughout much of the eastern half of North America (Tuskes et al. 1996, Wagner 2005). Caterpillars feed on members of 9 plant families, with notable genera including Betula, Prunus, Populus and Salix (Tuskes et al. 1996, Wagner 2005).

Morphology: Automeris io caterpillars are green with a white and pink stripe along their lateral sides and are covered in urticating, multi-branched scoli (Fig 5.13 a,b,d, Wagner 2005). Fifth instar caterpillars were, on average, $4.6 \mathrm{~cm}$ in length $(\mathrm{n}=5)$ at the time of experimentation, had rounded heads with an area of $23.81 \mathrm{~mm}^{2}$ (Fig $5.13 \mathrm{c}$ ) and smooth mandibles (Fig 5.13 e-h).

Behavioural Trials (Fig 5.13 i): Late instar caterpillars typically rested along the midvein on the underside of the leaves of hostplant prior to experimental trials. Anterior attacks caused the caterpillar to curl-in and thrash away from the pinch. When attacks continued the larva would curl-up by bringing the anterior and posterior ends together along the ventral side and fan out its scoli. This behaviour would make the areas protected by the caterpillars urticating scoli dominant and the ventral surface that lack scoli protected. No sound production was observed. As with its congener, I suspect the urticating scoli are the main defence in these caterpillars; when they were touched to the tough skin at the tips of the fingers it was slightly unpleasant and itchy, but when touched to the sensitive skin on the wrist there was noticeable inflammation accompanied by a burning sensation and extreme itch (personal observation). 


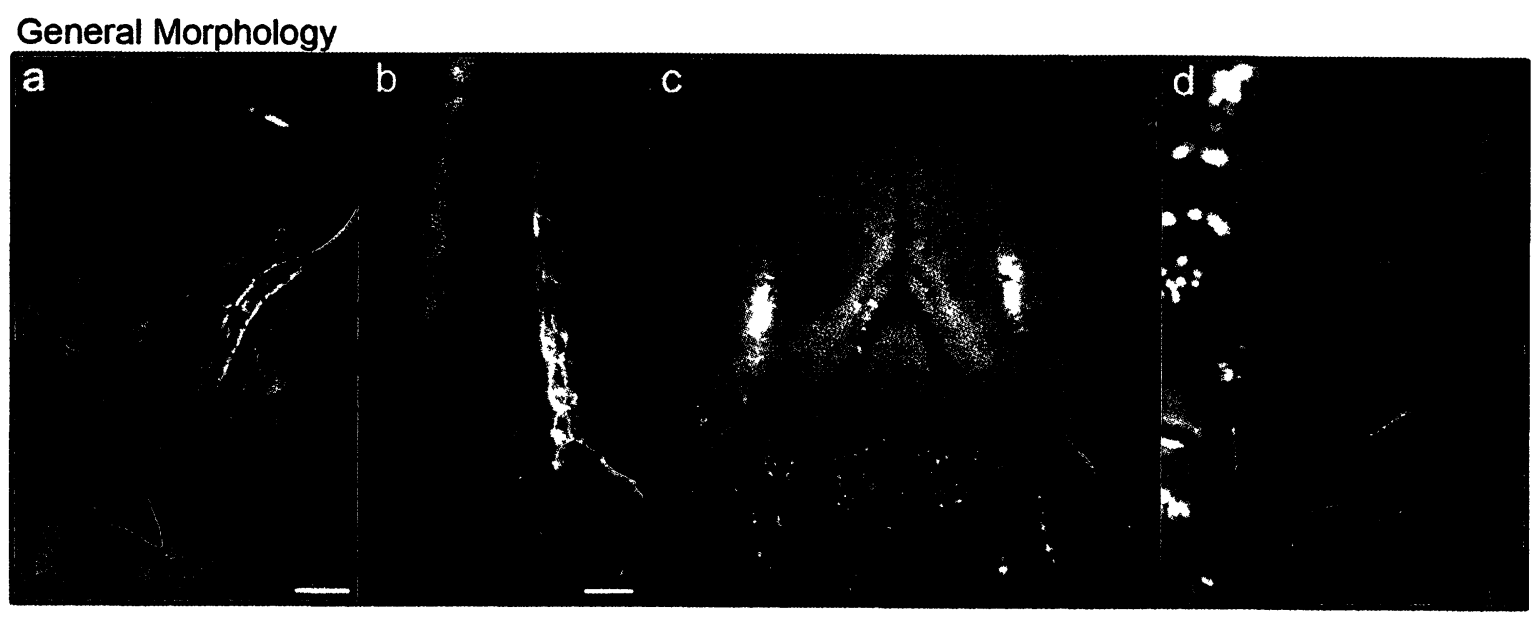

\section{Mandibles}

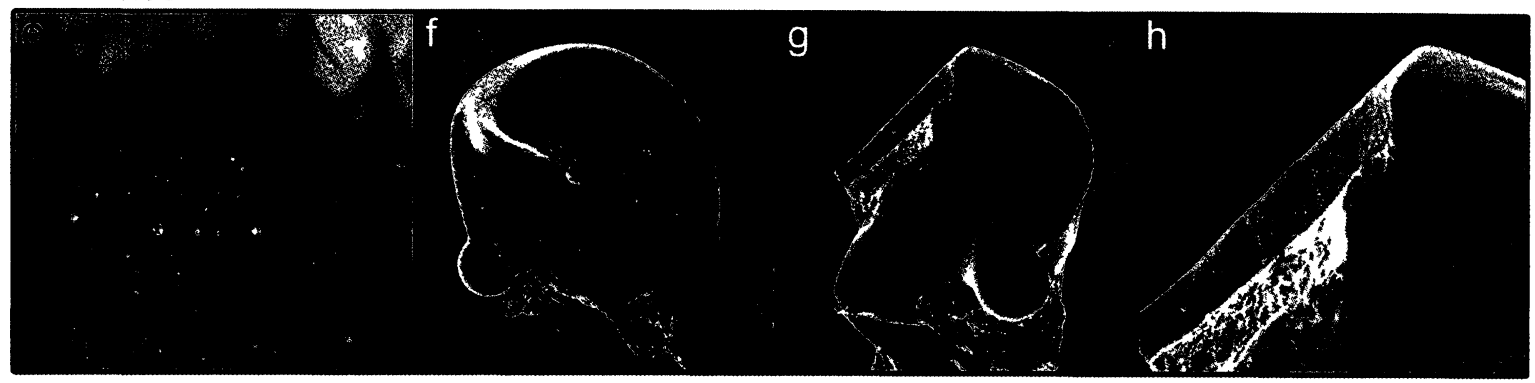

Defensive Behaviours

\begin{tabular}{|ll|}
\hline$\square$ Sound Production & $\square$ Biting \\
$\square$ Regurgitation & $\square$ Curling-in \\
$\square$ Chemical defense & $\square$ Curling up \\
$\square$ Thrashing & $\square$ Twitching \\
$\square$ Major thrashing & $\square$ Walk away \\
$\square$ Directed thrashing & $\square$ Dropping \\
\hline
\end{tabular}

Figure 5.13 Automeris io, the Io moth. General morphological features of a late instar caterpillar. (a) Lateral view of a late instar caterpillar on its hostplant, scale bar $1 \mathrm{~cm}$. (b) Dorsolateral view of a fifth instar caterpillar, scale bar $0.5 \mathrm{~cm}$. (c) Anterior view of the head capsule of a fifth instar, scale bar $0.5 \mathrm{~mm}$. (d) Close up of multi-branched urticating scoli, scale bar $0.5 \mathrm{~mm}$. Mandibles of a late instar caterpillar. (e) The mandibles of a fifth instar caterpillar in closed position, scale bar $0.5 \mathrm{~mm}$; (f) right mandible, inner face, scale bar $0.25 \mathrm{~mm}$; (g) left mandible, ventro-anterior view, scale bar $0.25 \mathrm{~mm}$; (h) close up of anterior edge of left mandible, scale bar $0.1 \mathrm{~mm}$. (i) List of defensive behaviours observed upon attack and when handled. All images taken by Veronica Bura, except (a) by Christina Borders (http://bugguide.net/node/view/82690/ bgimage) and (b) by Jayne Yack. 
Automeris phrynon Druce, Fig 5.14

Automeris phrynon is a new world moth found from Costa Rica to Ecuador (Marquis 1984, Savela 2009, Janzen and Hallwachs 2009). Caterpillars of this species feed on species from 13 different plant families (Janzen and Hallwachs 2009). Morphology: A phrynon larva was green, had a white longitudinal strip on the lateral side of its body and was covered in multi-branched urticating scoli (Fig 5.14 a,b). The specimen I tested was $5.2 \mathrm{~cm}$ in length at the time of experimentation.

Behavioural Trials (Fig 5.14 c): Prior to experimentation the caterpillar was resting on the dorsal surface of the leaf of its hostplant (Fig 5.14 a). Anterior attacks caused the caterpillar to fan out its scoli, presumably to cover more area with its chemical defence, and walked around the leaf as if it was attempting to get away from the attacking forceps. When attacked posteriorly the larva thrashed towards the site of attack and made quick, jerky movements from side to side over a very small distance. There is some debate among the three observers (A. Fleming, J. Yack and V. Bura) as to whether this species produced sound, but until more specimens have been tested I am reporting that the potential clicking sounds observed from the video were incidental noises from the movement of the caterpillar's scoli or thoracic legs and not intentional signals. Since it was not clear, I am counting this a negative for sound production. Therefore, no sound production was observed. As with the two other species in this subfamily I believe that the urticating scoli are the main defence in this species. 
General Morphology

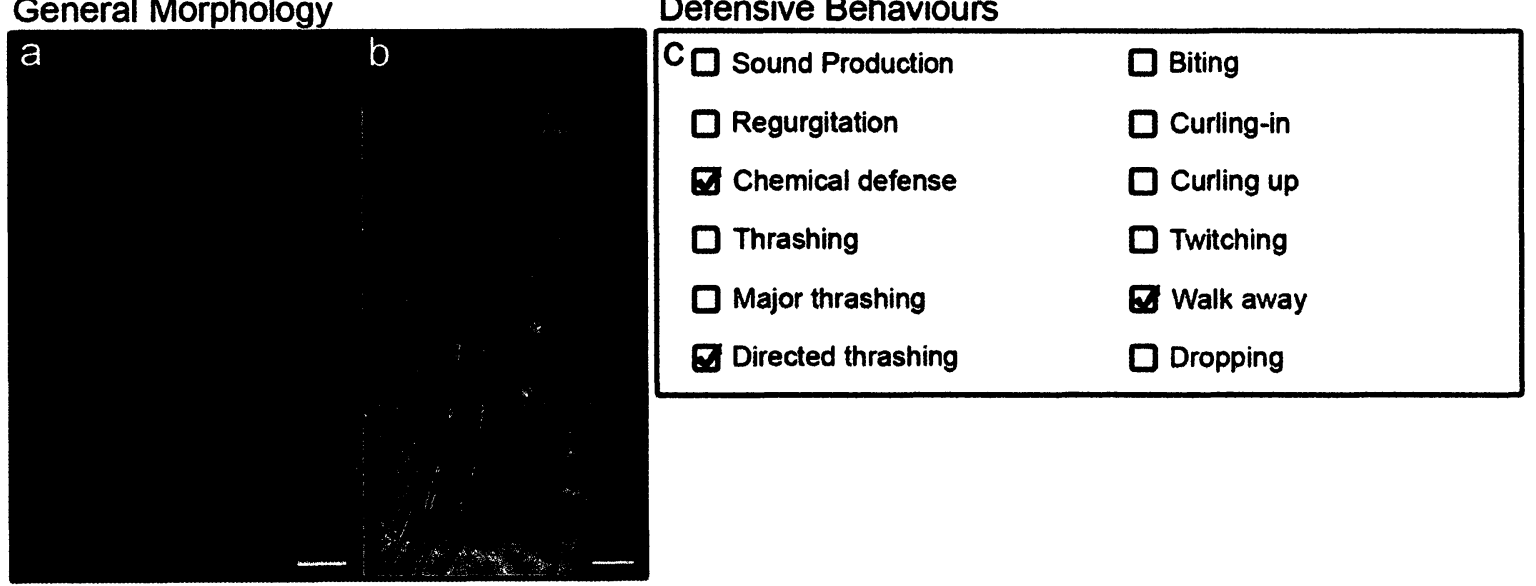

Figure 5.14 Automeris phrynon. General morphological features of a late instar caterpillar. (a) Dorsal view of a final instar caterpillar on its hostplant, scale bar $1 \mathrm{~cm}$. (b) Lateral view of a fifth instar caterpillar, scale bar $0.5 \mathrm{~cm}$. (c) List of defensive behaviours observed upon attack and when handled. Image in (a) by Jayne Yack and (b) by Veronica Bura. 


\section{SUBFAMILY: SATURNIINAE}

Caterpillars in this subfamily bear spiny scoli, some of which can house chemicals, although most are reduced to bumps in the late instars of some species (Tuskes et al. 1996, Deml and Dettner 2002).

Actias luna (Linnaeus), the Luna moth, Fig 5.15

The luna moth is a new world moth found throughout eastern North America (Tuskes et al. 1996, Wagner 2005). Caterpillars of this species feed on a variety of plants from five families including birch (Betula sp.), walnut (Juglans sp.), and hickory (Carya sp.) (Tuskes et al. 1996, Wagner 2005).

Morphology: Late instar Luna moth caterpillars are green with pink-red tubercles on each segment, a yellow line between each abdominal segment and a green head capsule (Fig $5.15 \mathrm{a}, \mathrm{b}$ ). At the time of experimentation, the caterpillars were about $5.3 \mathrm{~cm}$ in length $(\mathrm{n}=3)$, had a rounded head with an area of $26.04 \mathrm{~mm}^{2}$ (Fig $5.15 \mathrm{c}$ ) and ridged and serrated mandibles (Fig $5.15 \mathrm{~d}-\mathrm{g}$ ).

Behavioural Trials (Fig $5.15 \mathrm{~h}$ ): Caterpillars were resting on the underside of the leaf of hostplant, grasping the petiole, or on the twig prior to experimentation. When attacked anteriorly, the caterpillars responded by thrashing away from the pinch while clicking. This was followed by twitching and thrashing from side to side, and then regurgitating after a couple of pinches. Some caterpillars curled their heads in slightly, while others moved backwards a very short distance along the plant.

Acoustics: Caterpillars click in response to attack. 
Sound characteristics (Table 5.3): Luna moth caterpillars produced trains of clicks in response to attack by forceps (Fig $5.16 \mathrm{a}, \mathrm{b}$ ). The two-component clicks were $69.8 \pm 8.4$ ms long $(\mathrm{n}=9)$ and had a dominant frequency of $21.5 \pm 9.7 \mathrm{kHz}$ (Fig $5.16 \mathrm{c}, \mathrm{n}=14)$. Mechanism: Clicks were produced by the mandibles, likely by one contacting the other as they are closed.

Behaviours associated with sound production and proposed function: In animals that clicked and regurgitated (6/10), the clicks always preceded or accompanied regurgitation (Fig 5.15 i). This suggests that these signals function as an acoustic aposematic warning of an impending regurgitation, as previously hypothesized by Brown et al. (2007). 


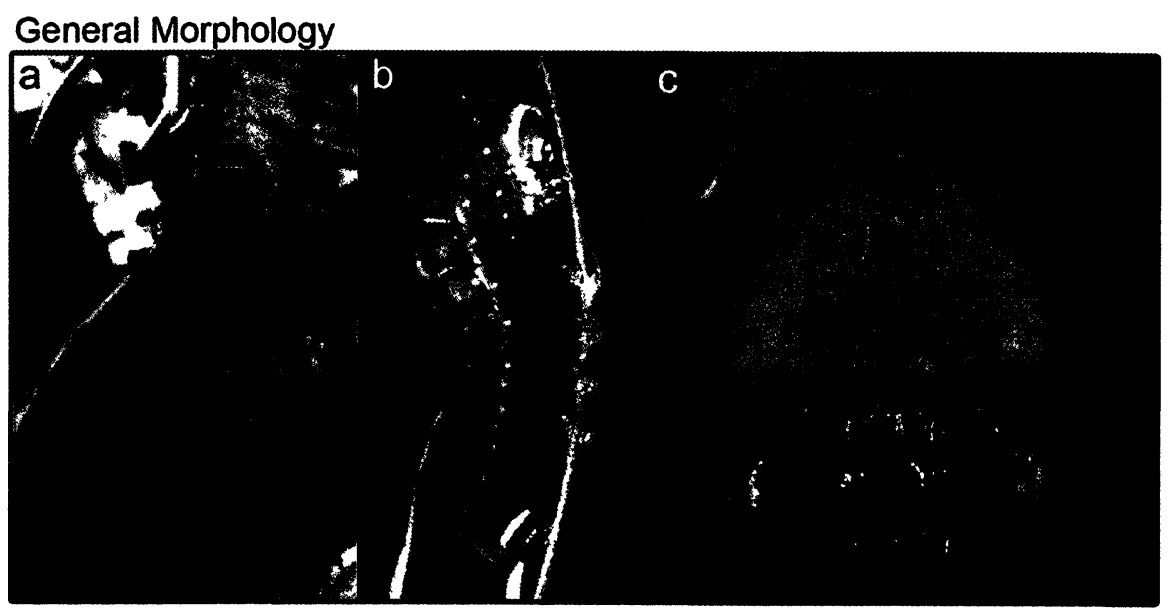

Mandibles

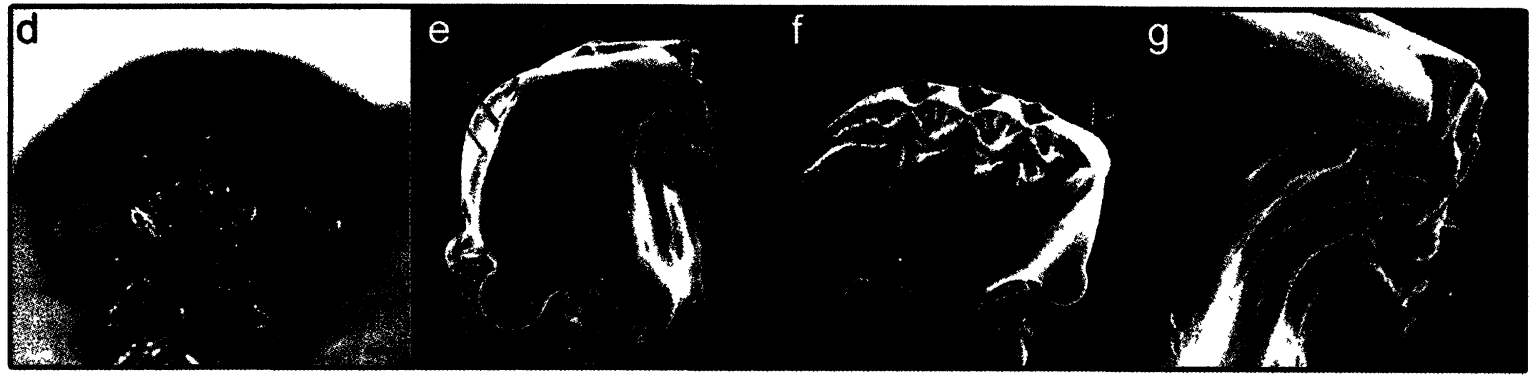

Defensive Behaviours

\begin{tabular}{|ll|}
\hline W Sound Production & $\square$ Biting \\
$\square$ Chemical defense & $\square$ Curling-in \\
$\square$ Thrashing & $\square$ Curling up \\
$\square$ Major thrashing & $\square$ Twitching \\
$\square$ Directed thrashing & $\square$ Walk away \\
\hline
\end{tabular}

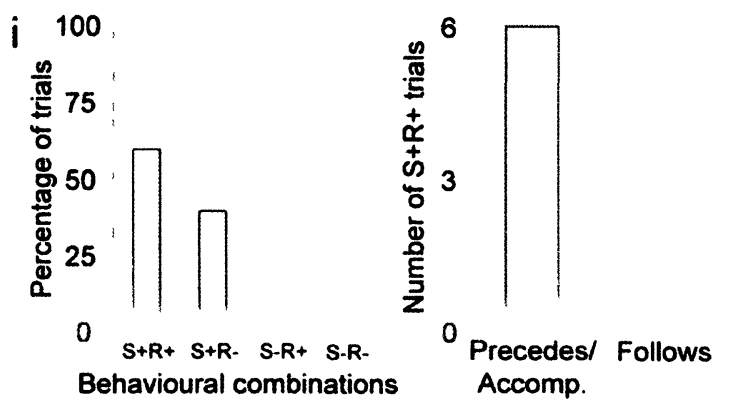

Figure 5.15 Actias luna, the Luna moth. General morphological features of a late instar caterpillar. (a) Dorso-lateral view of a late instar on its hostplant (anterior is down), scale bar $1 \mathrm{~cm}$. (b) Lateral view of a fifth instar larva, scale bar $0.5 \mathrm{~cm}$. (c) Anterior view of the head capsule of a fifth instar caterpillar, scale bar $0.5 \mathrm{~mm}$. Mandibles of a late instar caterpillar. (d) The mandibles of a fifth instar caterpillar in closed position, scale bar $0.5 \mathrm{~mm}$; (e) right mandible, ventro-anterior view, scale bar $0.25 \mathrm{~mm}$; (f) left mandible, inner face, scale bar $0.25 \mathrm{~mm}$; (g) close up of anterior edge of right mandible, scale bar $0.1 \mathrm{~mm}$. (h) List of defensive behaviours observed upon attack and when handled. (i) Histograms showing the relationship between sound production (S) and regurgitation (R) during the first 5 pinches of an attack $(n=10$, data from Sarah Brown). All images taken by Veronica Bura, except (b) by Sarah Brown. 
a
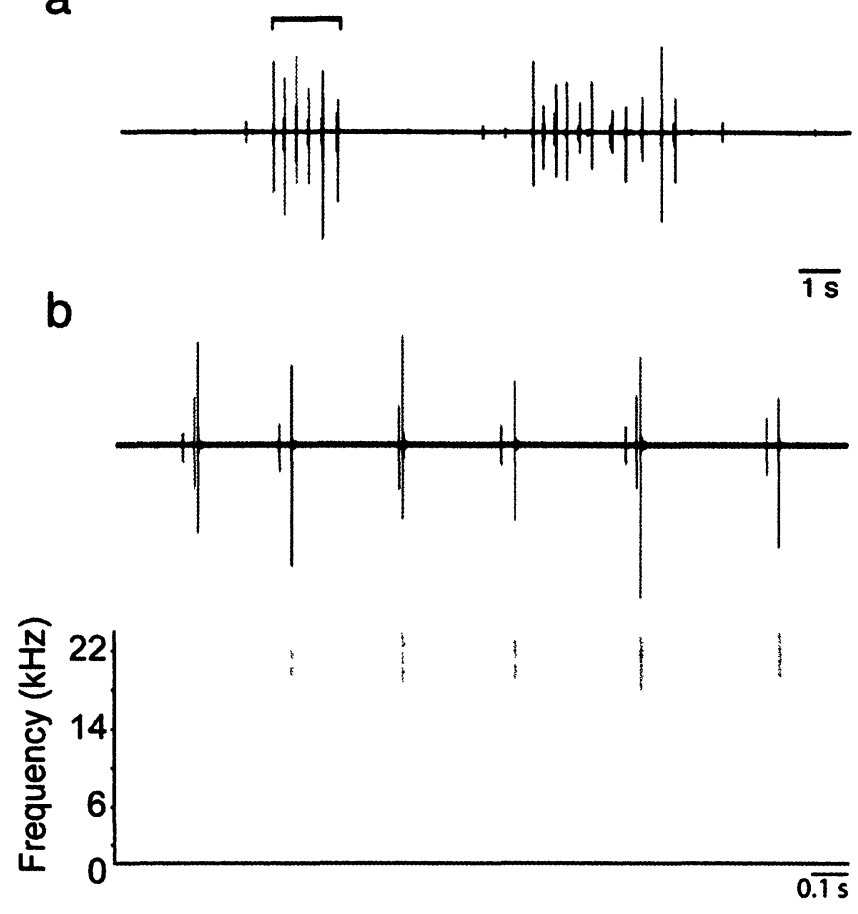

C

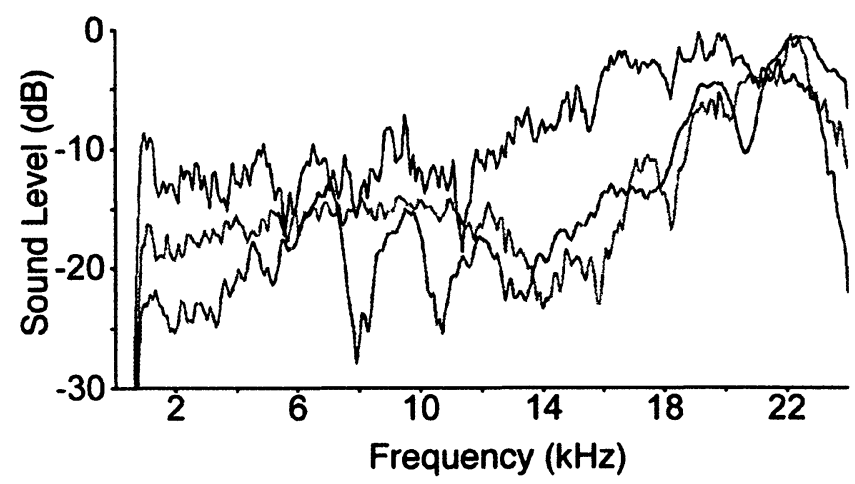

Figure 5.16 Sounds produced by late instar $A$. luna. (a) Oscillogram of clicks following attack. (b) Time expansion of bracket in (a) showing the multiple components of each click. The accompanying spectrogram shows the frequency distribution of each click. (c) Power spectra taken from three individuals. Please note that these sounds were sampled at a lower rate than those species that I recorded and so the spectrogram and power spectra have been cut off at a lower frequency and may not represent the full bandwidth of the signals. 
Antheraea pernyi (Guérin-Méneville), the Chinese Oak moth, Fig 5.17

The Chinese Oak moth is an old world moth found in eastern and central Asia (Mansingh 1972). Caterpillars feed on oak (Quercus sp.), beech (Fagus sp.), birch (Betula sp.) and several other plants (Pittaway 2010).

Morphology: Late instar Chinese Oak moth caterpillars are green, with a yellow stripe along their lateral sides and a spotted head capsule (Fig 5.17 a,b). At the time of experimentation the larvae were $4.15 \mathrm{~cm}$ in length $(\mathrm{n}=2)$, had rounded heads with an area of $27.05 \mathrm{~mm}^{2}$ (Fig $5.17 \mathrm{c}$ ) and ridged and serrated mandibles (Fig $5.17 \mathrm{~d}-\mathrm{g}$ ). Behavioural Trials (Fig 5.17 h): Caterpillars were resting on the underside of the leaves of hostplant or on the twig prior to experimentation. When attacked anteriorly the caterpillars thrashed away from the pinch and curled in, then thrashed from side to side and regurgitated on subsequent pinches. Sound production was not observed on tape, though clicking was observed when the animals were handled.

Acoustics: Due to the lack of recorded sounds, the characteristics of the acoustics are not yet known, though sounds appeared to be made by using the mandibles, as in other clicking species. The observation that these caterpillars regurgitate in response to attack suggests that like its congener Antheraea polyphemus, these larvae are also producing acoustic warning signals. However, future studies are needed to confirm this. 


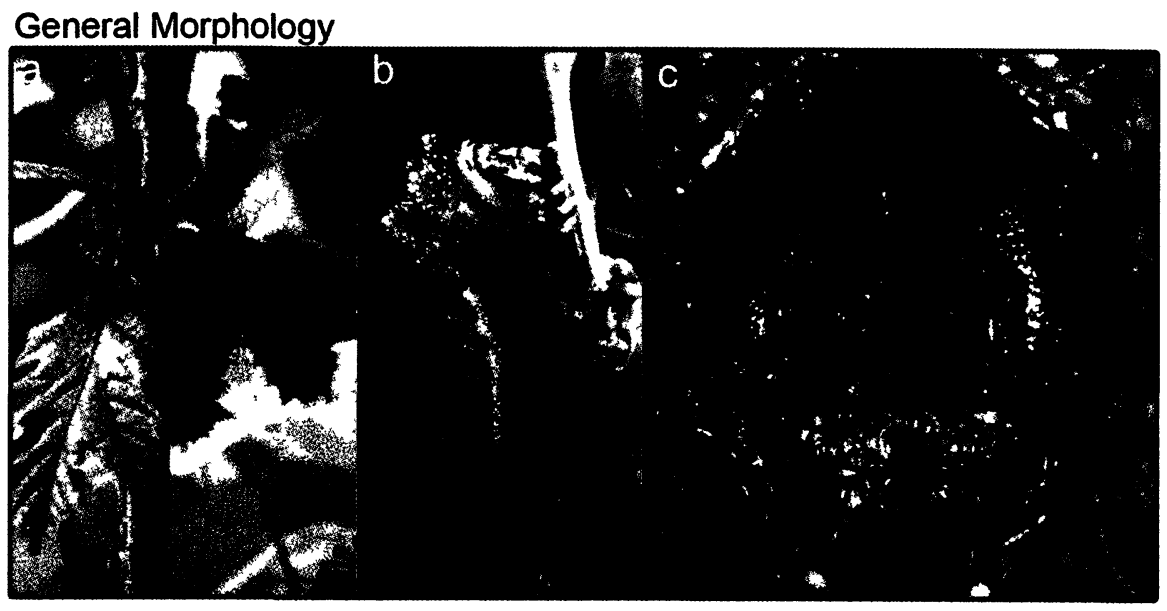

Mandibles

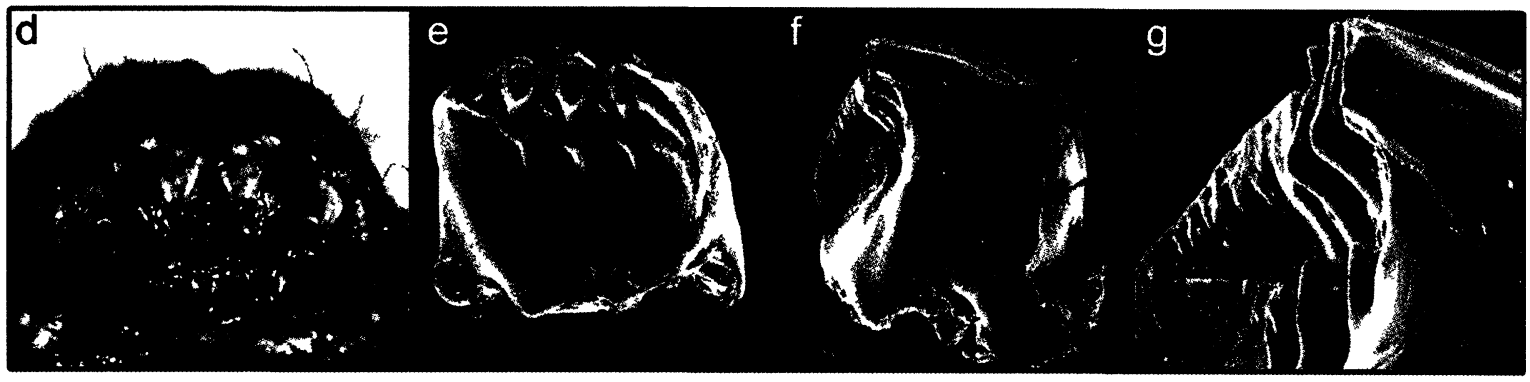

\section{Defensive Behaviours}

\begin{tabular}{|ll|}
\hline$\square$ Sound Production & $\square$ Biting \\
$\square$ Regurgitation & $\square$ Curling-in \\
$\square$ Chemical defense & $\square$ Curling up \\
$\square$ Thrashing & $\square$ Twitching \\
$\square$ Major thrashing & $\square$ Walk away \\
$\square$ Directed thrashing & $\square$ Dropping \\
\hline
\end{tabular}

Figure 5.17 Antheraea pernyi, the Chinese Oak moth. General morphological features of a late instar. (a) Antero-lateral view of a late instar caterpillar on its hostplant, scale bar $1 \mathrm{~cm}$. (b) Lateral view of a late instar larva, scale bar $0.5 \mathrm{~cm}$. (c) Anterior view of the head capsule of a fifth instar, scale bar $0.5 \mathrm{~mm}$. Mandibles of a late instar caterpillar. (d) The mandibles of a fifth instar in closed position, scale bar $0.5 \mathrm{~mm}$; (e) right mandible, inner face, scale bar $0.25 \mathrm{~mm}$; (g) left mandible, ventro-anterior view, scale bar $0.25 \mathrm{~mm}$; $(\mathrm{g})$ close up of anterior edge of left mandible, scale bar $0.1 \mathrm{~mm}$. (h) List of defensive behaviours observed upon attack and when handled. All images taken by Veronica Bura. 
Antheraea polyphemus (Cramer), the Polyphemus moth, Fig 5.18

The Polyphemus moth is a new world moth found throughout North America (Tuskes et al. 1996, Wagner 2005). Caterpillars of this species feed on plants from nine different families and include birch (Betula sp.), oak (Quercus sp.) and maple (Acer sp.) (Tuskes et al. 1996, Wagner 2005).

Morphology: Late instar caterpillars of A. polyphemus are large and green, with yellow stripes on their abdominal segments and a orange-brown head capsule (Fig 5.18 a,b). Caterpillars were about $6 \mathrm{~cm}$ long $(\mathrm{n}=3)$, had rounded heads with an area of $32.54 \mathrm{~mm}^{2}$ (Fig $5.18 \mathrm{c}$ ) and had ridged and serrated mandibles (Fig $5.18 \mathrm{~d}-\mathrm{g}$ ).

Behavioural Trials (Fig 5.18 h): Late instar larvae were resting on the underside of the leaves of hostplant prior to experimentation. When attacked anteriorly caterpillars thrashed away from the pinch, clicked and regurgitated. If regurgitation did not occur on the first pinch it occurred after subsequent pinches, as did some side to side thrashing and curling-in.

Acoustic: Caterpillars click when disturbed.

Sound characteristics (Table 5.3): Caterpillars produce trains of clicks that lasted over 1 min and contained 50-55 clicks (Fig 5.19 a,b). Individual clicks lasted $24.7 \pm 17.2 \mathrm{~ms}$ and contained multiple components. Clicks were broadband $(\mathrm{Q} 3=4.22 \pm 0.83, \mathrm{Q} 10=1.19 \pm$ $0.49, \mathrm{n}=15$ clicks from 3 animals) with a dominant frequency of $13.8 \pm 7.7 \mathrm{kHz}$ (Fig $5.19 \mathrm{c}, \mathrm{n}=30)$.

Mechanism: Video analysis confirmed older reports that the clicks emitted by the caterpillars were produced using their mandibles. As the ridges on the mandibles contact each other when they close they produce an audible click. 
Behaviours associated with sound production and proposed function: Brown determined that these clicks likely function as an acoustic aposematic warning due to the close relationship between sound and regurgitation (Fig 5.18 i). She found that the regurgitant had deterrent properties for both ants and mice, and suspects it would act similarly for birds. In trials with chickens, pecking by the bird always induced sound production and all larvae survived the trials. In another study the caterpillars were noted to click, thrash from side to side and regurgitate onto attacking chickadees (Heinrich and Collins 1983). The chickadees would sometimes kill the late instar caterpillars but they did not eat them, suggesting they may in fact be unpalatable to birds. 


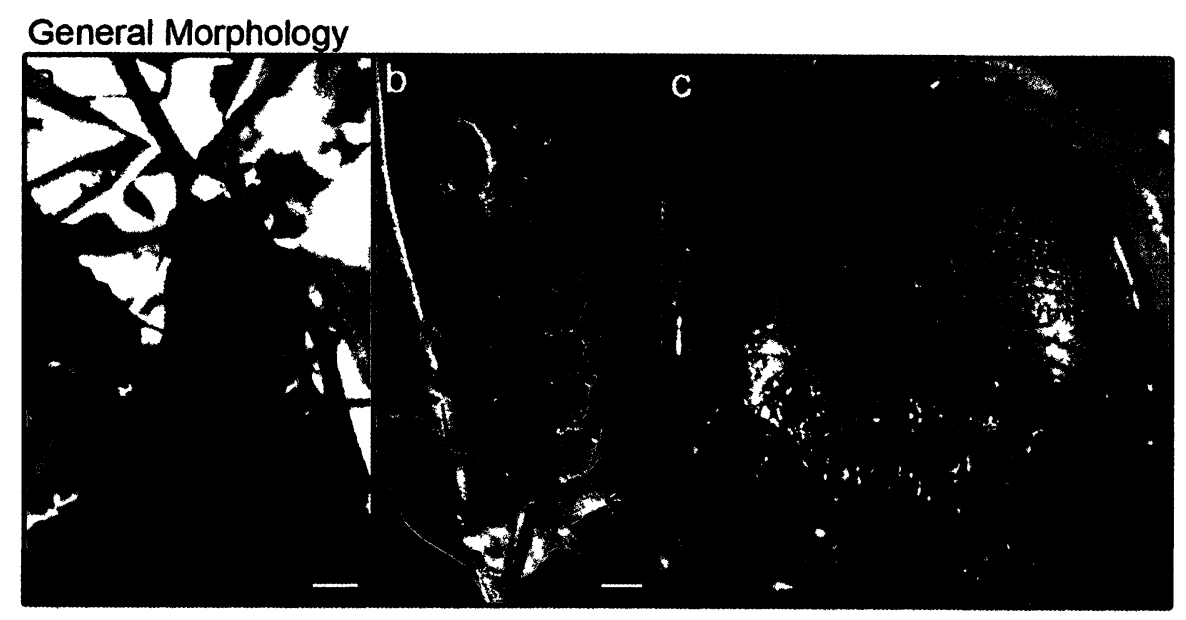

Mandibles

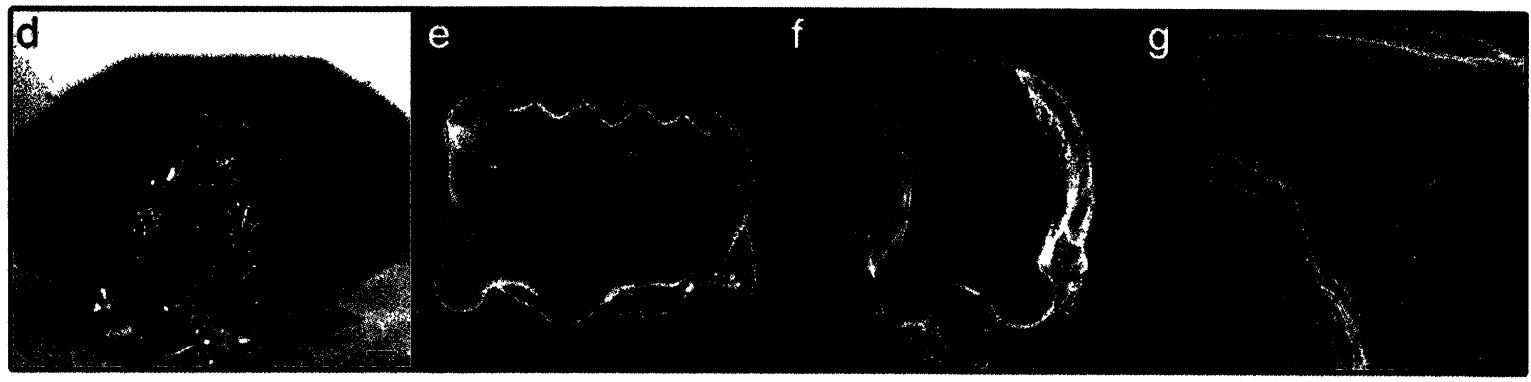

\section{Defensive Behaviours}

\begin{tabular}{|ll|}
\hline $\mathrm{W} \square$ Sound Production & $\square$ Biting \\
$\square$ Regurgitation & $\square$ Curling-in \\
$\square$ Chemical defense & $\square$ Curling up \\
$\square$ Thrashing & $\square$ Twitching \\
$\square$ Major thrashing & $\square$ Walk away \\
$\square$ Directed thrashing & $\square$ Dropping \\
\hline
\end{tabular}

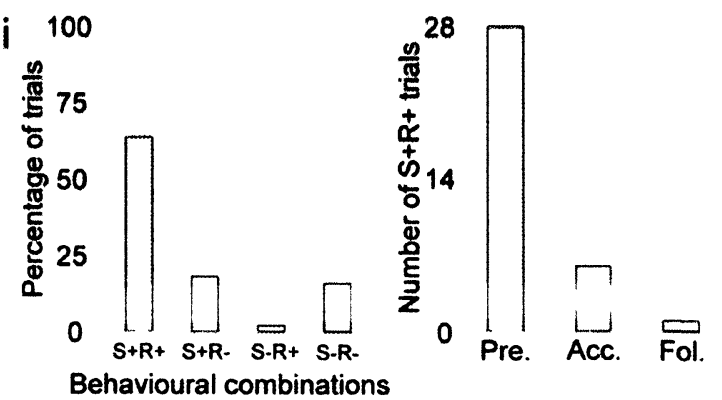

Figure 5.18 Antheraea polyphemus, the Polyphemus moth. General morphological features of a late instar caterpillar. (a) Lateral view of a late instar on its hostplant, scale bar $1 \mathrm{~cm}$. (b) Lateral view of a fifth instar caterpillar, scale bar $0.5 \mathrm{~cm}$. (c) Anterior view of the head capsule of a fifth instar caterpillar, scale bar $0.5 \mathrm{~mm}$. Mandibles of a late instar caterpillar. (d) The mandibles of a fifth instar in closed position, scale bar $0.5 \mathrm{~mm}$; (e) right mandible, inner face, scale bar $0.25 \mathrm{~mm}$; (f) left mandible, ventro-anterior view, scale bar $0.25 \mathrm{~mm}$; (g) close up of anterior edge of left mandible, scale bar $0.1 \mathrm{~mm}$. (h) List of defensive behaviours observed upon attack and when handled. (i) Histograms showing the relationship between sound production (S) and regurgitation (R) during the first 5 pinches of an attack $(n=50$, data from Sarah Brown). All images by Veronica Bura, except (a) by Gary Pignato (http://bugguide.net/node/view/131717/bgimage) and (b) by Sarah Brown. 
a

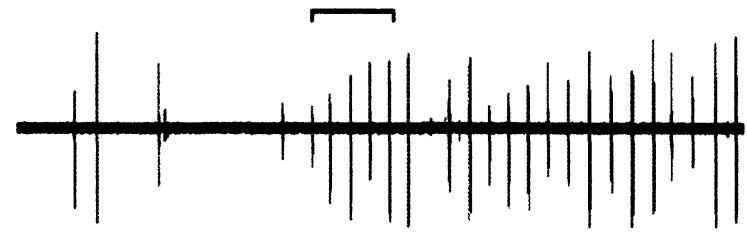

b

$\overline{1 \mathrm{~s}}$
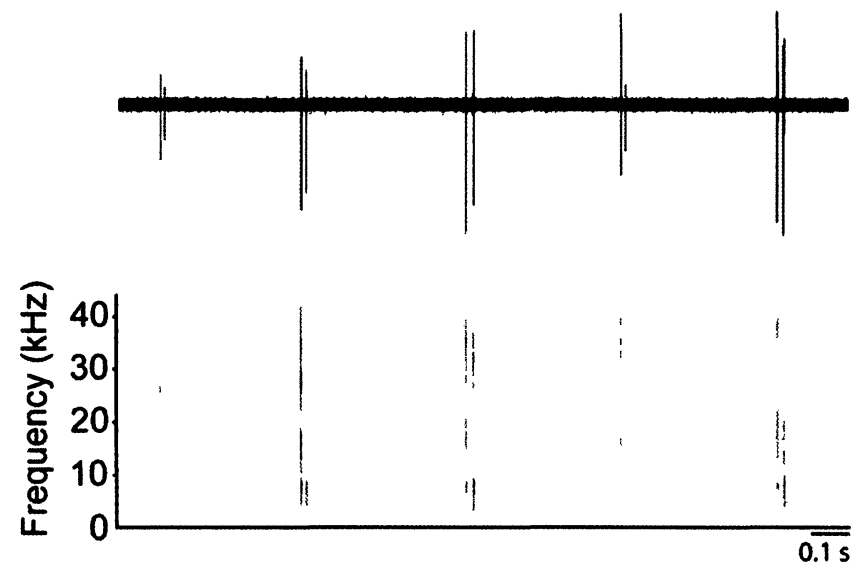

C

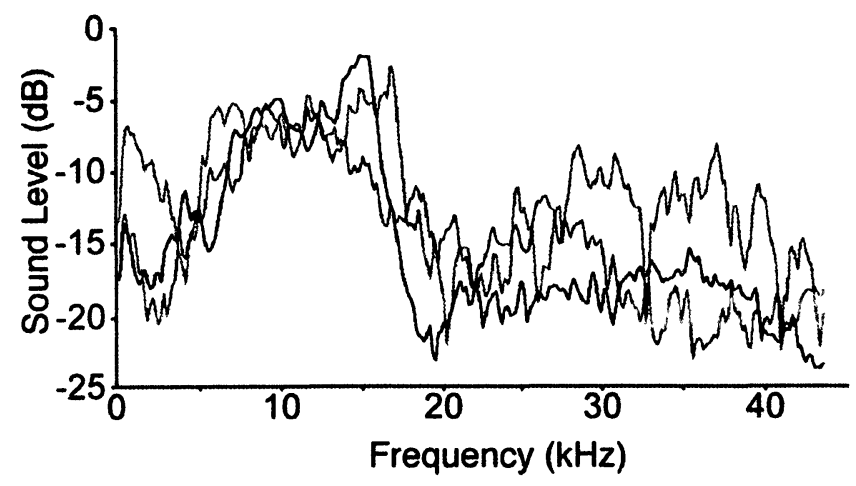

Figure 5.19 Sounds produced by late instar $A$. polyphemus. (a) Oscillogram of clicks following attack. (b) Time expansion of bracket in (a) showing the multiple components of each click. The accompanying spectrogram shows the frequency distribution of each click. (c) Power spectra taken from three individuals (modified from Brown et al. (2007)). 
Attacus atlas (Linnaeus), the Atlas moth, Fig 5.20

Attacus atlas is an old world moth found throughout south-eastern Asia and the Indo-Pacific islands (Peigler 1989). Caterpillars of this species feed on plants from 46 different families including Oleaceae (olive), Rosaceae (rose) and Betulaceae (birch) (Peigler 1989).

Morphology: The large caterpillars of the Atlas moth are green, covered in long scoli and a white, waxy bloom (Fig $5.20 \mathrm{a}, \mathrm{b}$ ). The fifth instar larva was about $8.9 \mathrm{~cm}$ in length at the time of experimentation, had a rounded head with an area of $43.04 \mathrm{~mm}^{2}$ (Fig 5.20 c) and smooth mandibles (Fig $5.20 \mathrm{e}-\mathrm{h}$ ).

Behavioural Trials (Fig 5.20 i): Caterpillars were resting on a bare twig of hostplant prior to experimentation. Anterior attacks caused the larvae to curl-in, thrash away from the pinch and secrete a foul-smelling liquid from their scoli. No sound production was observed. The secretions of Atlas moth caterpillars, which can be discharged as droplets or sprayed at a distance, contain biogenic amines, histamine and acetylcholine and have been shown to have a weak deterrent effect on ant predators (Deml and Dettner 1994). Deml and Dettner (1994) suggest that the main target of these secretions are avian predators based on some components of the secretions, the mode of delivery and the irritant properties as felt by humans. In another study pieces of caterpillar were offered to captive quails and whole dead caterpillars to wild jackdaws (Deml and Dettner 1995). The quails increased their handling time and grooming behaviours, whereas the jackdaws picked off the head of the caterpillar and ate the insides, leaving almost the entire integument intact, showing that the secretions do have an effect on avian predators. 


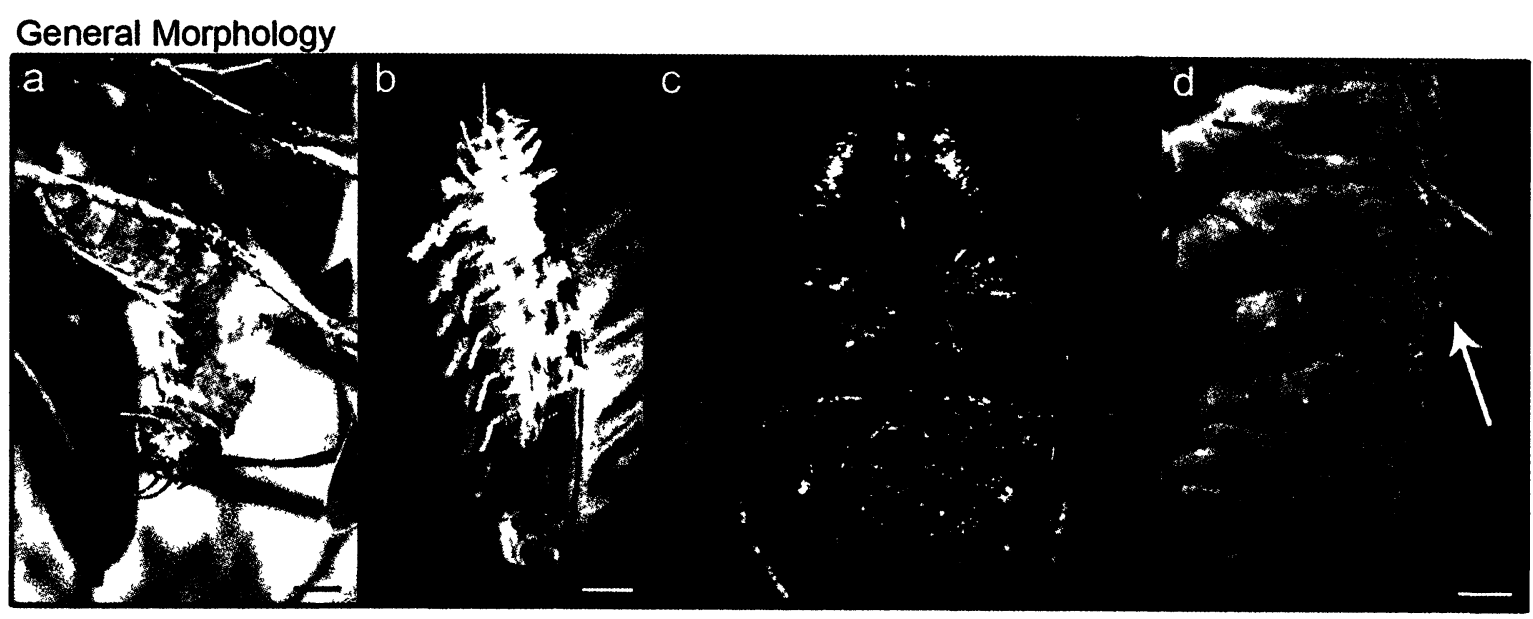

Mandibles

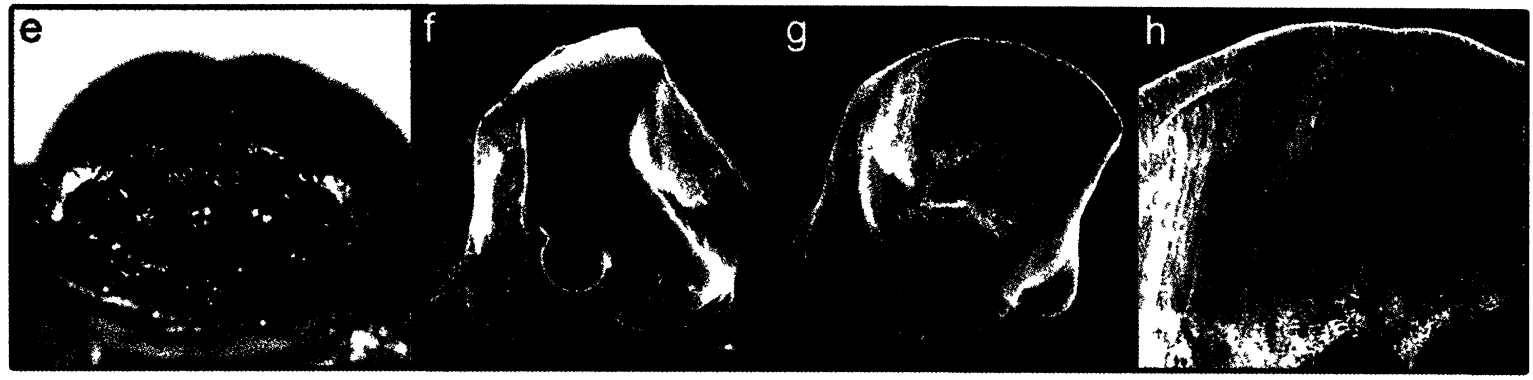

Defensive Behaviours

\begin{tabular}{|ll|}
\hline i $\square$ Sound Production & $\square$ Biting \\
$\square$ Regurgitation & $\square$ Curling-in \\
$\square$ Chemical defense & $\square$ Curling up \\
$\square$ Thrashing & $\square$ Twitching \\
$\square$ Major thrashing & $\square$ Walk away \\
$\square$ Directed thrashing & $\square$ Dropping \\
\hline
\end{tabular}

Figure 5.20 Attacus atlas, the Atlas moth. General morphological features of a late instar caterpillar. (a) Lateral view of a fifth instar caterpillar resting on its hostplant, scale bar $1 \mathrm{~cm}$. (b) Lateral view of a fifth instar caterpillar, scale bar $1 \mathrm{~cm}$. (c) Anterior view of the head capsule of a fifth instar, scale bar $0.5 \mathrm{~mm}$. (d) Close up of scolus secretions (arrow), scale bar $0.5 \mathrm{~cm}$. Mandibles of a late instar caterpillar. (e) The mandibles of a fifth instar caterpillar in closed position, scale bar $0.5 \mathrm{~mm}$; (f) right mandible, ventro-anterior view, scale bar $0.5 \mathrm{~mm}$; (g) left mandible, inner face, scale bar $0.5 \mathrm{~mm}$; (h) close up of anterior edge of left mandible, scale bar $0.1 \mathrm{~mm}$. (i) List of defensive behaviours observed upon attack and when handled. All images taken by Veronica Bura, except (a) by Ron Yeo (http://2.bp.blogspot.com/_pbKM4qxm q4c/SeYPmj0L2VI/AAAAAAAAI_0/MBlg2Ntk1ek/s400/14.jpg) and (b) by Antoine Hnain. 
Callosamia promethea (Drury), the Promethea moth, Fig 5.21

The Promethea moth is a new world moth found throughout eastern North America (Tuskes et al. 1996, Wagner 2005). Caterpillars of this species feed on plants from several families including Oleaceae and Rosaceae (Tuskes et al. 1996, Wagner 2005).

Morphology: Final instar Promethea caterpillars are pale-green with four red scoli on their dorsal thoracic segments and one yellow scolus on their posterior end (Fig $5.21 \mathrm{a}, \mathrm{b}$ ). They are covered in black dots and have a yellow head and anal plate. At the time of experimentation, fifth instar caterpillars were $5.2 \mathrm{~cm}$ in length $(\mathrm{n}=5)$, had rounded heads with an area of $12.45 \mathrm{~mm}^{2}$ (Fig $5.21 \mathrm{c}$ ) and slightly serrated mandibles (Fig $5.21 \mathrm{~d}-\mathrm{g}$ ). Behavioural Trials (Fig 5.21 h): Caterpillars were resting on the underside of the leaf along the mid-vein prior to experimentation. When attacked anteriorly the caterpillars responded by curling-in and regurgitating on subsequent pinches. Posterior attacks resulted in slight directed thrashing towards the site of attack (more like raising its head dorsally off the leaf) followed by regurgitation and some quiet clicking. Acoustics: The sounds produced by the Promethea caterpillar were intermittent quiet clicks.

Sound characteristics: Promethea clicks did not occur in trains (Fig 5.22 a). Individual clicks were $71.38 \pm 132.36 \mathrm{~ms}$ in duration and contained $1-2$ components (Fig $5.22 \mathrm{~b}, \mathrm{n}=$ 6 clicks from 1 animal). Clicks were broadband $(\mathrm{Q} 3=4.22 \pm 0.83, \mathrm{Q} 10=1.19 \pm 0.49, \mathrm{n}$ $=5$ clicks from 1 animal) and had a mean dominant frequency of $34.08 \pm 7.07 \mathrm{kHz}$ (Fig $5.22 \mathrm{c})$ 
Mechanism: Video analysis revealed that clicks were produced using the mandibles. Clicks are likely produced as the anterior edge of one mandible contacts the other as the mandibles as closed.

Behaviours associated with sound production and proposed function: Regurgitation was observed in all trials, whereas clicking occurred in $8 / 20$ trials (Fig 5.21 i). Of those 8 trials clicking followed regurgitation in all but one trial. Based on these observations I would argue that sound production in C. promethea caterpillars may be incidental and not a true signal. It is also interesting to note that this species was previously identified as a non-sound producer by another student (A. Hnain), so the behaviour may not be typical of all populations, though it should be noted that those tested in my study were from two different populations. Sounds produced by these caterpillars may represent an example of a 'precursor' to more ritualized signals and will be discussed further in part B. 


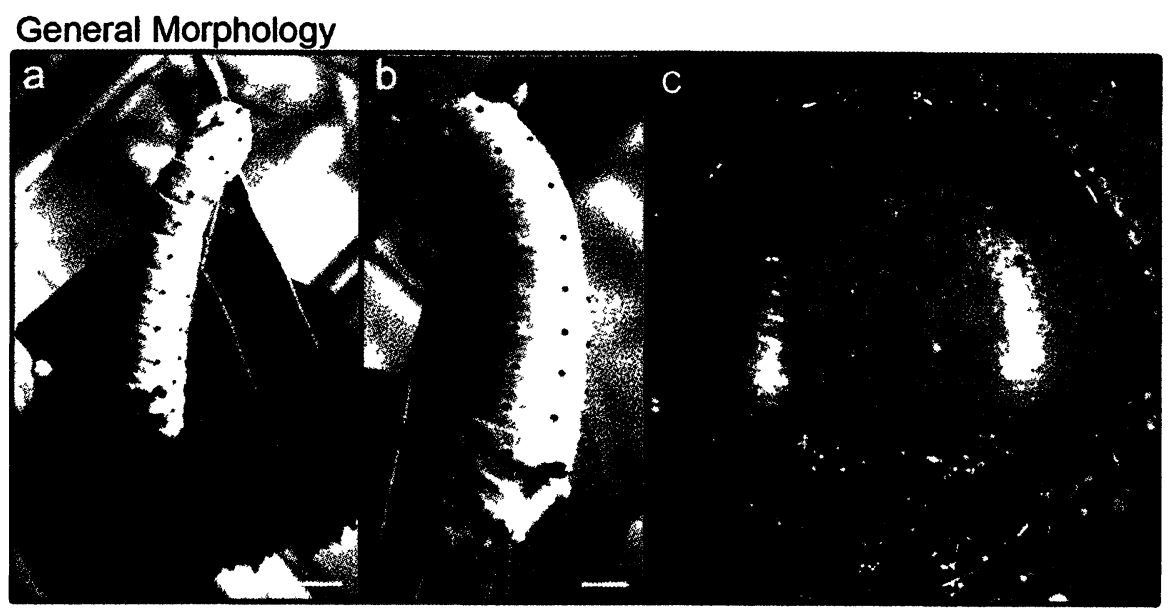

\section{Mandibles}

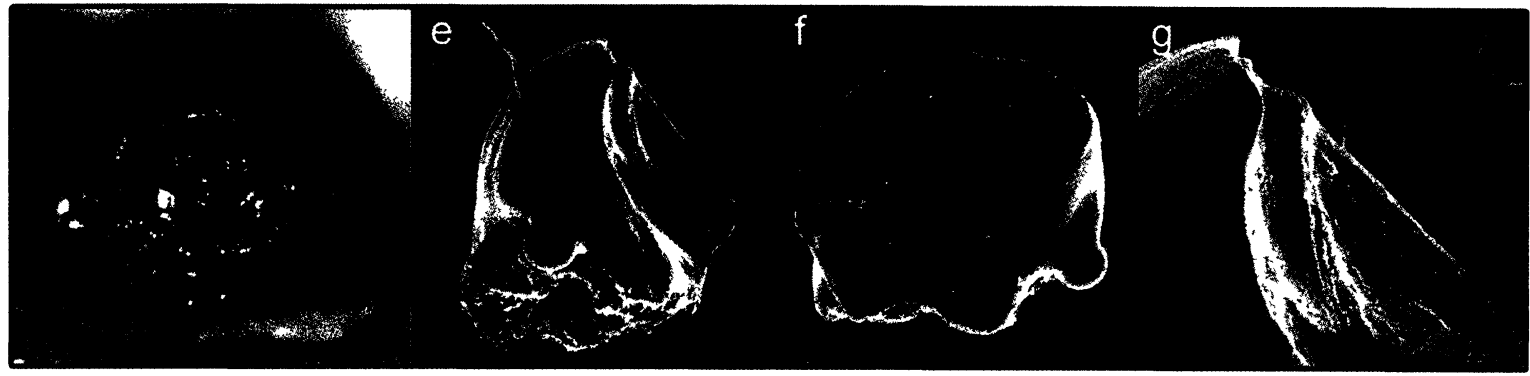

Defensive Behaviours

\begin{tabular}{|ll|}
\hline h \\
$\square$ Sound Production & $\square$ Biting \\
$\square$ Chemical defense & $\square$ Curling-in \\
$\square$ Thrashing & $\square$ Curling up \\
$\square$ Major thrashing & $\square$ Twitching \\
$\square$ Directed thrashing & $\square$ Walk away \\
\hline
\end{tabular}

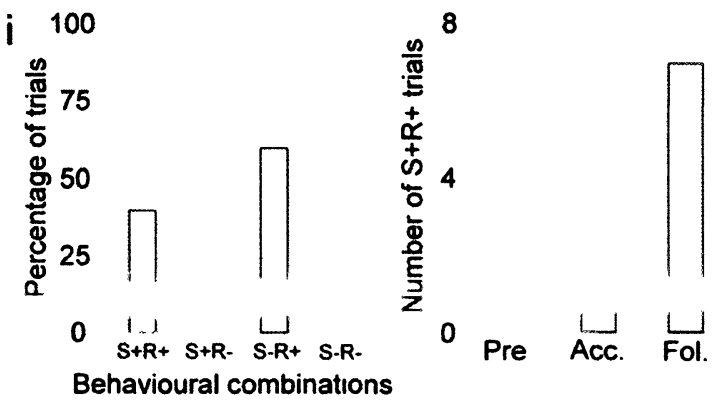

Figure 5.21 Callosamia promethea, the Promethea moth. General morphological characteristics of a late instar caterpillar. (a) Dorsal view of a fifth instar on its hostplant, anterior is down, scale bar $0.75 \mathrm{~cm}$. (b) Lateral view of a fifth instar caterpillar, anterior is down, scale bar $0.5 \mathrm{~cm}$. (c) Anterior view of the head capsule of a fifth instar larva, scale bar $0.5 \mathrm{~mm}$. Mandibles of a late instar caterpillar. (d) The mandibles of a final instar in closed position, scale bar $0.5 \mathrm{~mm}$; (e) right mandible, ventro-anterior view, scale bar $0.25 \mathrm{~mm}$; (f) left mandible, inner face, scale bar 0.25 $\mathrm{mm}$; (g) close up of anterior edge of right mandible, scale bar $0.1 \mathrm{~mm}$. (h) List of defensive behaviours observed upon attack and when handled. (i) Histograms showing the relationship between sound production (S) and regurgitation (R) during the first 5 pinches of an attack $(n=$ 20). All images taken by Veronica Bura. 
a

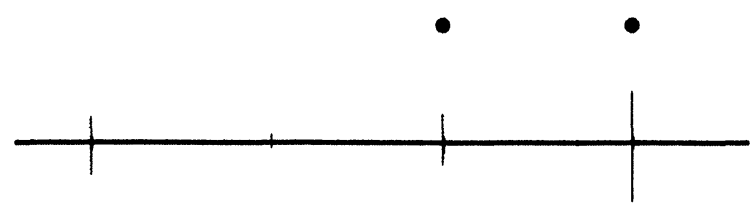

b

$\overline{1 s}$
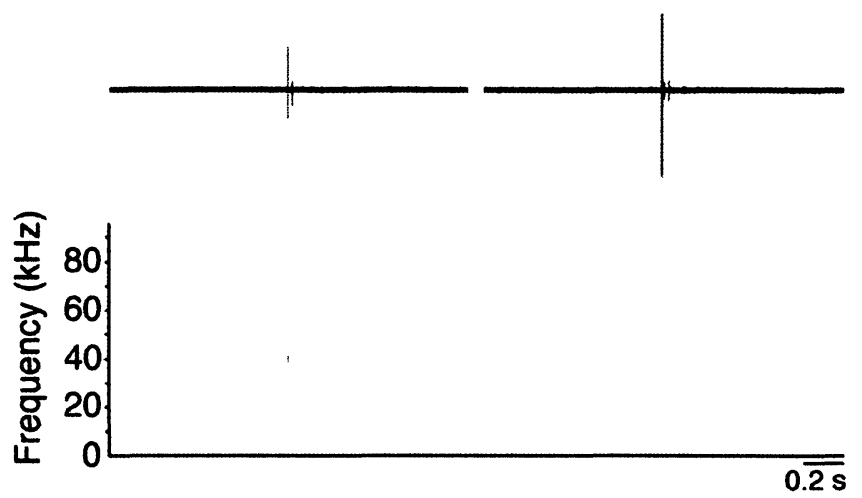

C

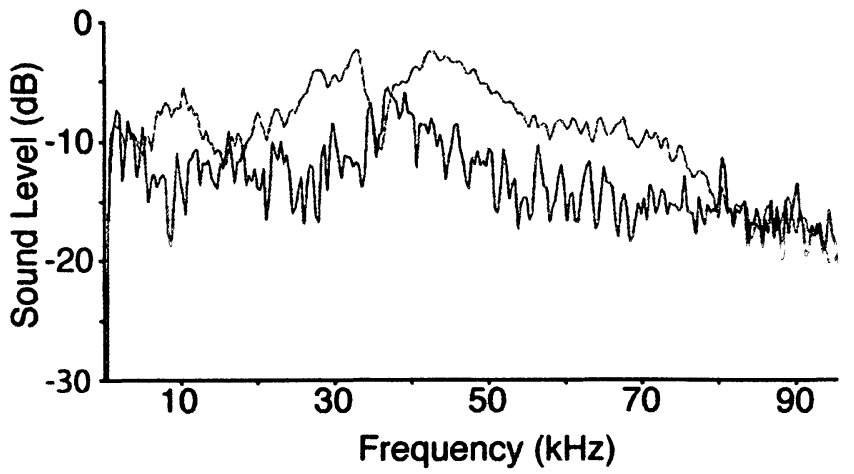

Figure 5.22 Sounds produced by late instar $C$. promethea. (a) Oscillogram of clicks following attack. (b) Time expansion of clicks indicated by black circles in (a) showing the multiple components of each click. The accompanying spectrogram shows the frequency distribution of each click. (c) Power spectra taken from two individuals. 
Saturnia pyri (Denis \& Schiffermüller), the Great Peacock moth, Fig 5.23

The Great Peacock moth is an old world moth found throughout southern Europe, Asia Minor, and Northern Africa (Rougeot 1971). Caterpillars feed on plant species from 10 different families including Rosaceae, Juglandaceae and Betulaceae (Pittaway 2010). Morphology: Saturnia pyri caterpillars are large, green and covered in colourful scoli (Fig $5.23 \mathrm{a}, \mathrm{b}$ ). Late instar larvae were about $7 \mathrm{~cm}$ in length at the time of experimentation, had rounded heads with an area of $31.04 \mathrm{~mm}^{2}$ (Fig $5.23 \mathrm{c}$ ) and serrated mandibles (Fig $5.23 \mathrm{e}-\mathrm{h}$ ).

Behavioural Trials (Fig 5.23 i): Late instar larvae were resting on the underside of the leaves of their hostplant along the petiole prior to experimentation. Anterior attacks caused the larvae to curl-in, chirp and secrete chemicals from its anterior scoli. When attacked posteriorly the caterpillars would thrash towards the site of attack, chirp and secrete chemicals from both the anterior and posterior scoli. The secretions were accompanied by a foul-smelling odor.

Acoustics: Details on the sound characteristics, mechanism and associated behaviours have been reported in Bura et al. (2009) and Chapter 3 of this thesis but are presented here for comparative purposes.

Sound characteristics (Table 5.3): Giant Peacock moth caterpillars produced trains of chirps that lasted $1.7 \pm 1.12 \mathrm{~s}$ and contained $5.74 \pm 3.45$ chirps (Fig $5.24 \mathrm{a}, \mathrm{b}, \mathrm{n}=42$ ). Chirps could be divided into two types (Fig 5.24 c). Type 1 chirps were longer, $67.48 \pm$ $23.15 \mathrm{~ms}$, occurred at the beginning of a train, had a mean dominant frequency of $34.45 \pm$ $11.93 \mathrm{kHz}(\mathrm{n}=25)$ and were broadband $(\mathrm{Q} 3=4.97 \pm 0.44, \mathrm{Q} 10=3.07 \pm 1.17, \mathrm{n}=15$ chirps from 3 animals). Type two chirps were shorter, $9.14 \pm 10.17 \mathrm{~ms}$, occurred at the 
end of a train, had a mean dominant frequency of $21.57 \pm 15.19 \mathrm{kHz}(\mathrm{n}=23)$ and were more broadband than type $1(\mathrm{Q} 3=1.43 \pm 0.16, \mathrm{Q} 10=1.10 \pm 0.40, \mathrm{n}=15$ chirps from 3 animals).

Mechanism: Video analysis revealed that stridulation was achieved using the mandibles. The anterior edge of one mandible slides against the inner face of the other mandible to produce chirps.

Behaviours associated with sound production and proposed function: The appearance of chemical secretions was correlated with the production of sound (Fig $5.23 \mathrm{j}$ ). Of 20 trials, 18 had both sound production and chemical secretion and in 16 of those trials sound production preceded or accompanied the secretion. This close association suggests that chirps function as an acoustic warning signal for the scoli-secretions. In other studies these chemicals have been shown to have deterrent properties against ants, microorganisms, fungi and birds (Deml and Dettner 1993, 1995, Deml 2001). 
General Morphology

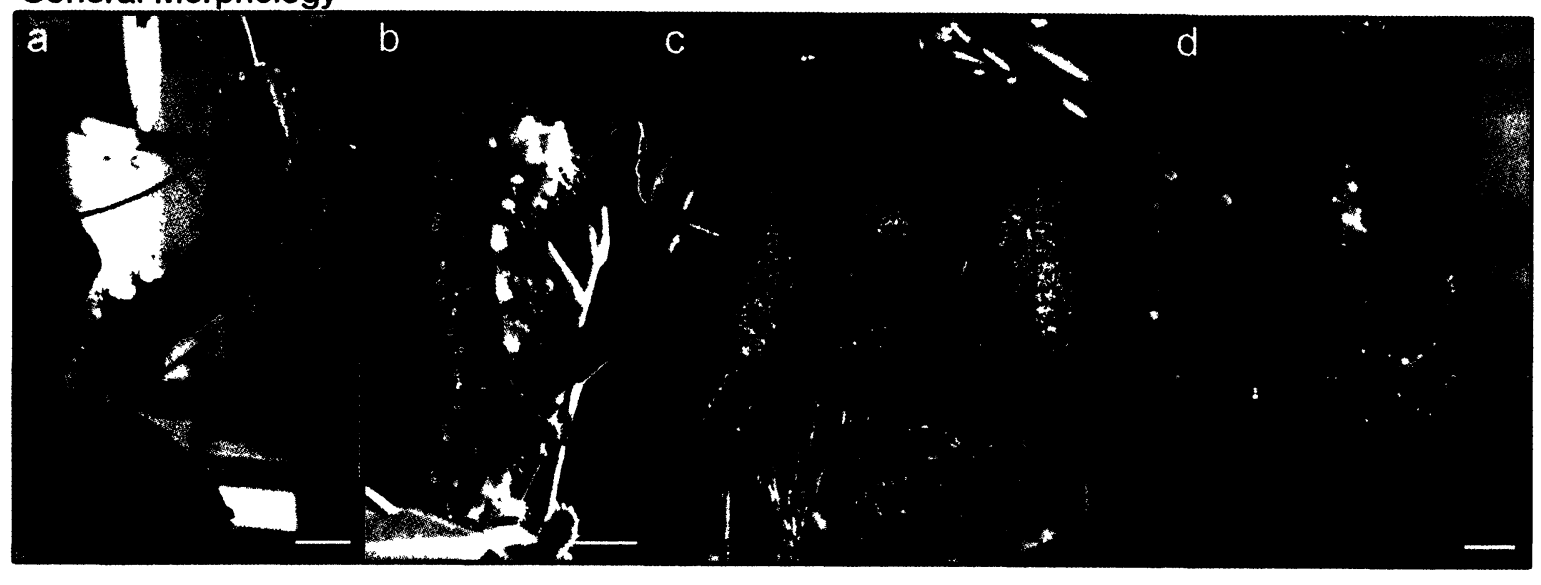

Mandibles

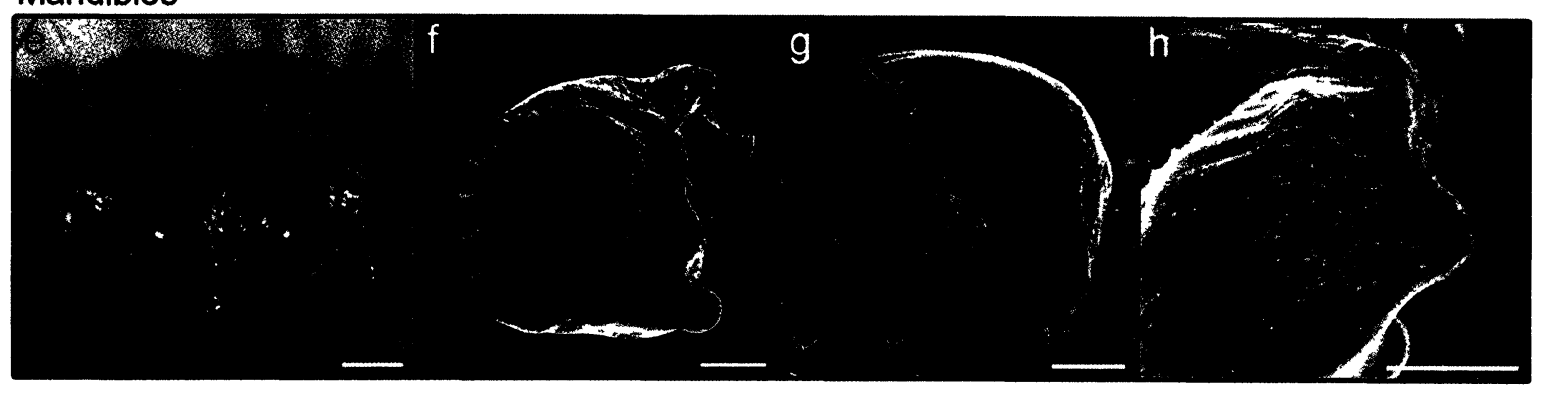

Defensive Behaviours

\begin{tabular}{|ll|}
\hline $\mathrm{i} \square$ Sound Production & $\square$ Biting \\
$\square$ Regurgitation & $\square$ Curling-in \\
$\square$ Chemical defense & $\square$ Curling up \\
$\square$ Thrashing & $\square$ Twitching \\
$\square$ Major thrashing & $\square$ Walk away \\
$\square$ Directed thrashing & $\square$ Dropping \\
\hline
\end{tabular}

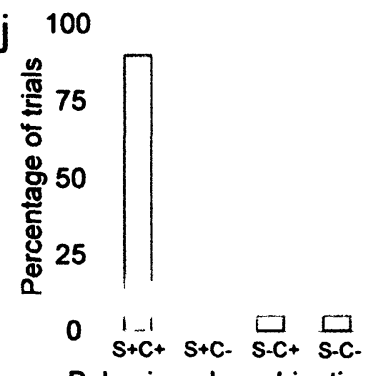

Behavioural combinations

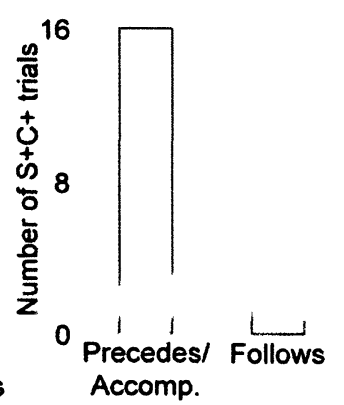

Figure 5.23 Saturnia pyri, the Great Peacock moth. General morphological characteristics of a late instar caterpillar. (a) Dorso-lateral view of a late instar caterpillar on its hostplant, scale bar 1 $\mathrm{cm}$. (b) Lateral view of a late instar, scale bar $1 \mathrm{~cm}$. (c) Anterior view of the head capsule of a late instar larva, scale bar $0.5 \mathrm{~mm}$. (d) Close up of scolus showing defensive secretions, scale bar 0.5 $\mathrm{mm}$. Mandibles of a late instar caterpillar. (e) The mandibles of a late instar larva in closed position, scale bar $1 \mathrm{~mm}$; (f) right mandible, inner face, scale bar $0.5 \mathrm{~mm}$; (g) left mandible, ventro-anterior view, scale bar $0.5 \mathrm{~mm}$; (h) close up of textured inner face of right mandible, scale bar $0.5 \mathrm{~mm}$. (i) List of defensive behaviours observed upon attack and when handled. (j) Histograms showing the relationship between sound production (S) and chemical secretion (C) during the first 5 pinches of an attack $(n=20)$. All images taken by Veronica Bura. 
a

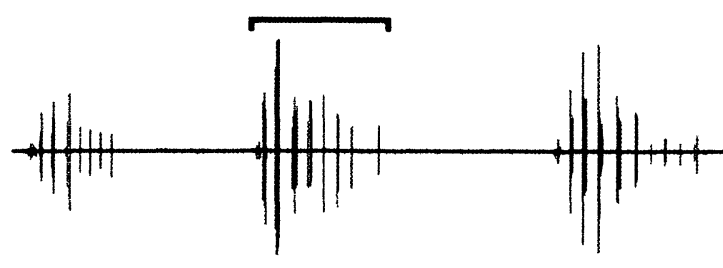

b
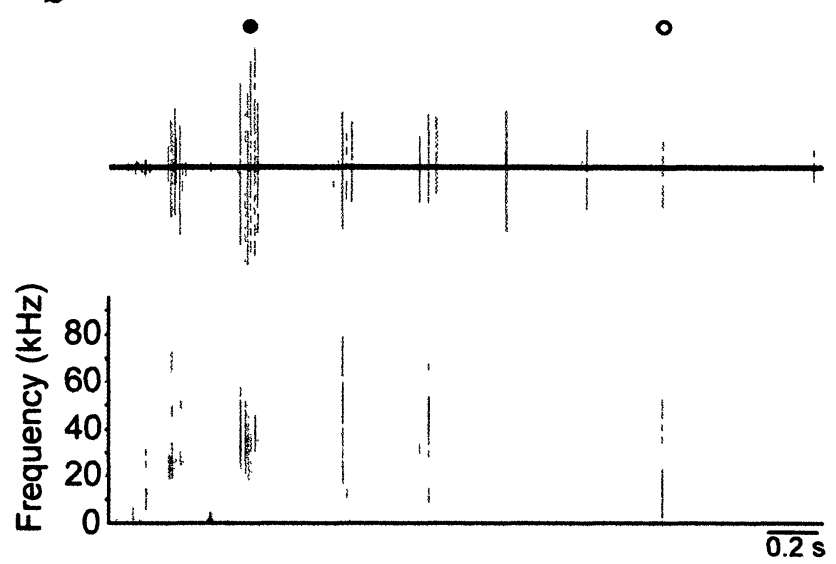

C Multi-component

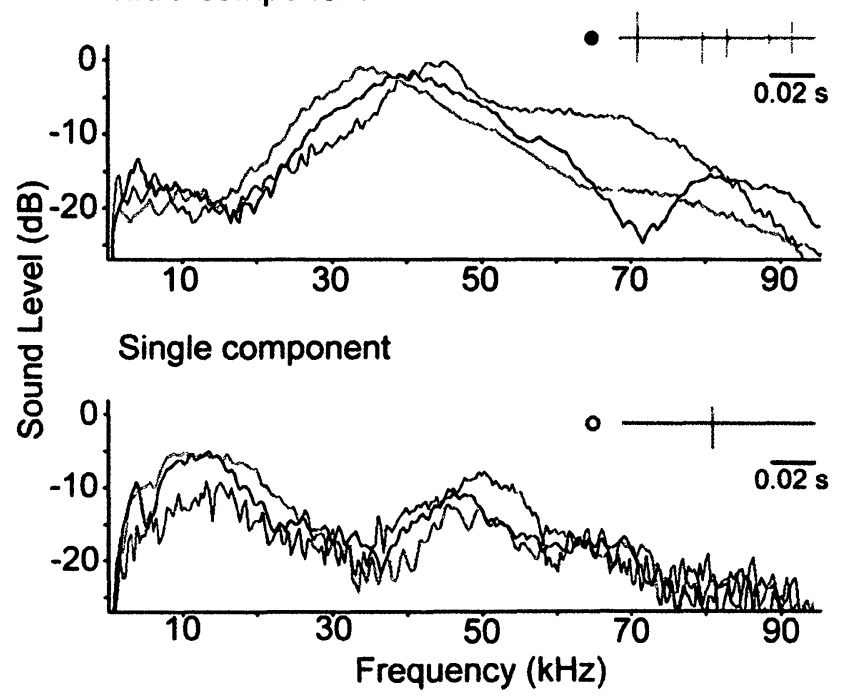

Figure 5.24 Sounds produced by late instar S. pyri. (a) Oscillogram of chirps following attack. (b) Time expansion of the bracketed train from part (a), showing typical multicomponent (closed circle) and single component (open circle) chirps. The accompanying spectrogram shows the frequency distribution of each chirp. (c) Power spectra of multicomponent and single component chirps taken from three individuals. Time expanded chirps from (b) are inset for each type. 


\section{FAMILY: SPHINGIDAE}

This family encompasses 1400 species from three subfamilies, Smerinthinae, Sphinginae and Macroglossinae (Kawahara et al. 2009). Results are presented here for 8 species from Smerinthinae, 10 from Sphinginae and 9 from Macroglossinae.

\section{SUBFAMILY: SMERINTHINAE}

The principle defence for the majority of species in this subfamily is crypsis and many larvae are countershaded (Kitching and Cadiou 2000).

Adhemarius ypsilon (Rothschild \& Jordan), Fig 5.25

Adhemarius ypsilon is a new world moth found from northern Mexico through Central America to Venezuela (Oehkle 2009, Savela 2009). This species feeds on members of the Laurel family (Lauraceae), including 11 species of Ocotea sp. (Janzen and Hallwachs 2009).

Morphology: Caterpillars of $A$. ypsilon can be green or yellow (Oehkle 2009). The single specimen that I tested was green (Fig 5.25 a), $8.1 \mathrm{~cm}$ in length, had a triangular head with an area of $28.88 \mathrm{~mm}^{2}$ (Fig $5.25 \mathrm{c}$ ) and smooth mandibles with a blunt, sculpted ridge on its inner face (Fig $5.25 \mathrm{~d}-\mathrm{g}$ ).

Behavioural trials (Fig 5.25 h): The A. ypsilon larva was positioned on what would have been the underside of the leaf (plant was eaten), securing itself to the plant using its prolegs and claspers to grip the stem and petiole. When attacked anteriorly the caterpillar first thrashed away from the pinch and subsequently thrashed from side to side at a rate of about 2.95 flicks per second, in a wide arc from proleg 4. Attacks directed at the posterior end elicited similar behaviours with the first thrash being towards the site of attack instead of away from it. Once the caterpillar had been attacked a few times it would begin 
thrashing when the forceps came close to it, making it difficult to deliver successive pinches. No sound production was observed. Thrashing, in general but especially at high speeds, would likely discourage continued attack by some predators as it would be very time consuming and potentially costly. 


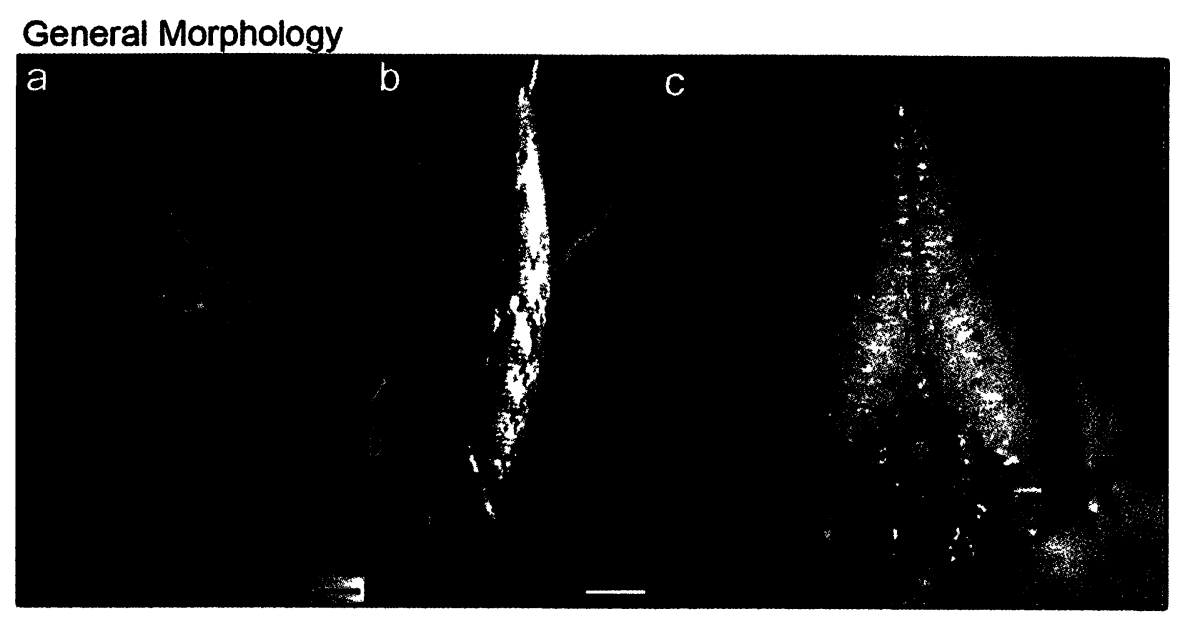

Mandibles

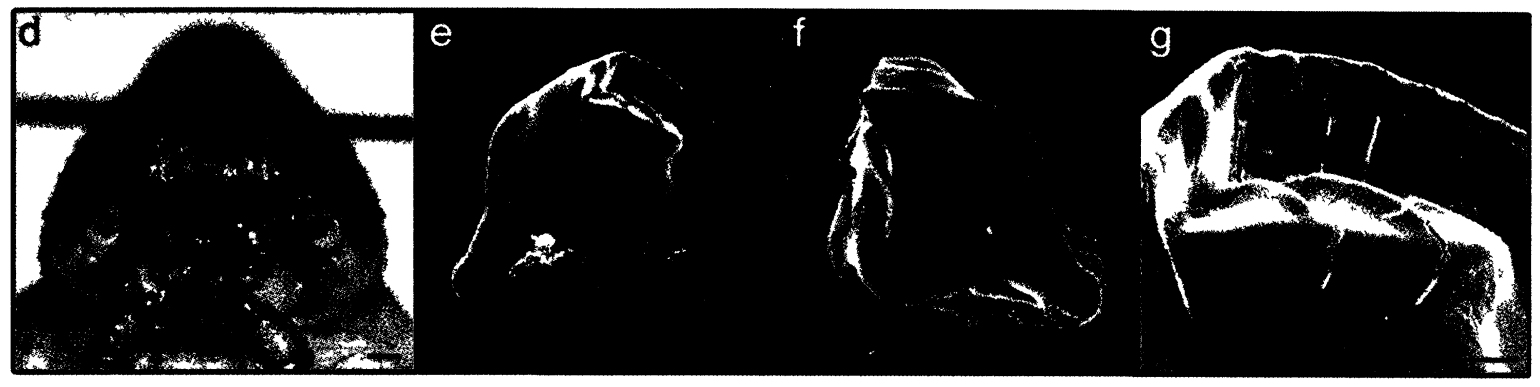

Defensive Behaviours

\begin{tabular}{|ll|}
\hline$\square$ Sound Production & $\square$ Biting \\
$\square$ Regurgitation & $\square$ Curlıng-in \\
$\square$ Chemical defense & $\square$ Curling up \\
$\square$ Thrashing & $\square$ Twitching \\
$\square$ Major thrashing & $\square$ Walk away \\
$\square$ Directed thrashing & $\square$ Dropping \\
\hline
\end{tabular}

Figure 5.25 Adhemarius ypsilon. General morphological features of a late instar caterpillar. (a) Lateral view of a fifth instar on a sprig of hostplant, scale bar $1 \mathrm{~cm}$. (b) Dorso-lateral view of a fifth instar caterpillar, scale bar $1 \mathrm{~cm}$. (c) Anterior view of the head capsule of a fifth instar, scale bar $0.5 \mathrm{~mm}$. Mandibles of a late instar caterpillar. (d) The mandibles of a late instar in closed position, the labrum has been removed, scale bar $0.5 \mathrm{~mm}$; (e) right mandible, inner face, scale bar $0.25 \mathrm{~mm}$; (f) left mandible, ventral-anterior view, scale bar $0.25 \mathrm{~mm}$; (g) close up of anterior edge and blunt ridge on inner face of right mandible, scale bar $0.1 \mathrm{~mm}$. (h) List of defensive behaviours observed upon attack and when handled. All images taken by Veronica Bura except (a) by Jayne Yack and (b) by Dan Janzen. 


\section{Amorpha juglandis (JE Smith), the Walnut sphinx, Fig 5.26}

The Walnut sphinx is a new world moth found throughout eastern and central North America (Wagner 2005, Tuttle 2007). Caterpillars feed on butternut and walnut (Juglans sp.), hickory (Carya sp.), hop hornbeam (Ostrya sp.), American hornbeam (Carpinus caroliniana), hazelnut (Corylus sp.), alder (Alnus sp.) and beech (Fagus sp.) (Wagner 2005, Tuttle 2007).

Morphology: Walnut sphinx caterpillars can vary in colour from green to reddish-brown and are covered in white granules (Tuttle 2007). The broods that I reared consisted solely of green caterpillars (Fig 5.26 a,b) and were very difficult to find on the plant. Fifth instar larvae were approximately $4.5 \mathrm{~cm}$ in length, had triangular heads with an area of 20.53 $\mathrm{mm}^{2}$ (Fig $5.26 \mathrm{c}$ ), and smooth mandibles (Fig $5.26 \mathrm{e}-\mathrm{h}$ ).

Behavioural Trials (Fig 5.26 i): Wagner (2005) noted that when disturbed, A. juglandis caterpillars thrash violently from side to side and produce sound. During the day, larvae rest along the midrib of the leaf (Fig 5.26 a, Wagner 2005). I did not record any instances of violent thrashing in this species, though, when handled it would eagerly bite and regurgitate, thrashing around to gain a new point of 'attack'. When attacked anteriorly or posteriorly the caterpillar would respond with directed thrashing and sound production. Acoustic: Details on the sound characteristics, mechanism and associated behaviours have been reported in Chapter 4 but are presented here for comparative purposes. Sound characteristics (Table 5.3): The acoustic signals produced by A. juglandis consist of trains of whistles (Fig 5.27 a). Trains were on average $2626 \pm 2339 \mathrm{~ms}$ in duration and contained 1-8 whistles ( $\mathrm{n}=30$ trains from 10 animals). Whistles were on average $440 \pm$ $272 \mathrm{~ms}$ in duration $(\mathrm{n}=101)$. Whistles could be divided into three types $(1,2,3)$ and had 
different spectral characteristics (see Table 4.1, Fig 5.17 b,c); types 1 and 2 were broadband while type 3 was narrowband.

Mechanism: Through the use of selective spiracular obstructions, laser vibrometry and high speed videography it was confirmed that the sounds of these caterpillars are produced by the movement of air through the last abdominal spiracle (Fig $5.26 \mathrm{~d}$ ), as discussed earlier in Chapter 4.

Behaviours associated with sound production and proposed function: Whistles were produced when the caterpillar was attacked. Though they did regurgitate when handled for extended periods, this regurgitation was not at all closely associated with sound production (Fig $5.26 \mathrm{j}$ ). Sounds were produced in conjunction with a longitudinal contraction of the body and likely function to startle vertebrate predators, like birds (see Chapter 4 for additional detail). 


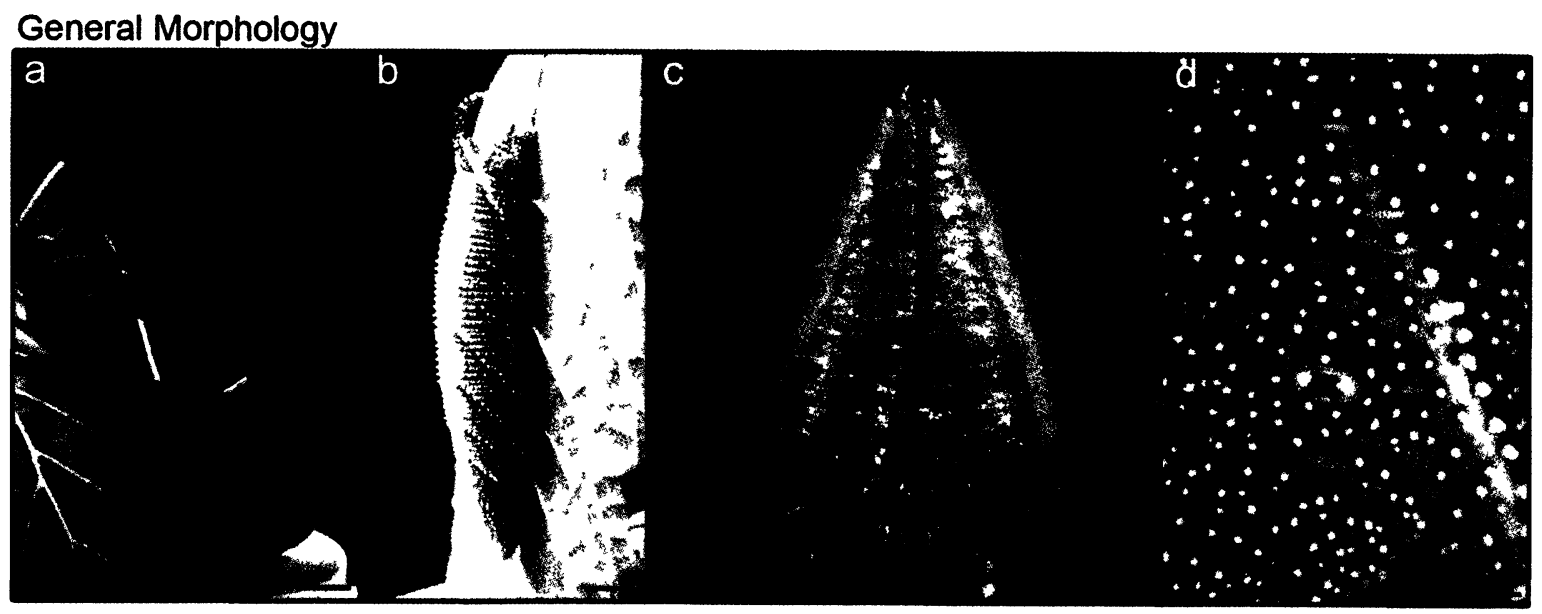

Mandibles

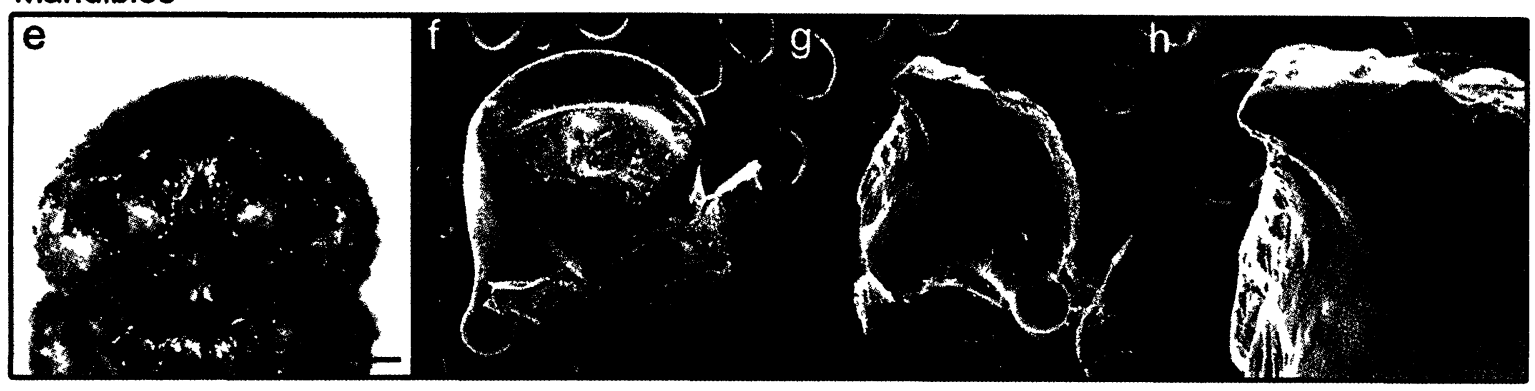

Defensive Behaviours

\begin{tabular}{|ll|}
\hline i $\square$ Sound Production & $\square$ Biting \\
$\square$ Regurgitation & $\square$ Curling-in \\
$\square$ Chemical defense & $\square$ Curling up \\
$\square$ Thrashing & $\square$ Twitching \\
$\square$ Major thrashing & $\square$ Walk away \\
$\square$ Directed thrashing & $\square$ Dropping \\
\hline
\end{tabular}

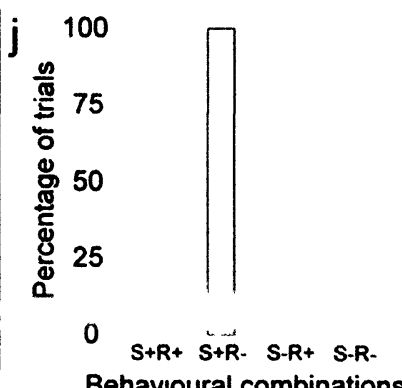

Figure 5.26 Amorpha juglandis, the Walnut sphinx. General morphological features of a late instar caterpillar. (a) Lateral view of a fifth instar caterpillar resting on its hostplant, scale bar 1 $\mathrm{cm}$. (b) Lateral view of a fifth instar caterpillar, scale bar $0.5 \mathrm{~cm}$. (c) Anterior view of the head capsule of a fifth instar, scale bar $0.5 \mathrm{~mm}$. (d) Close up of $8^{\text {th }}$ abdominal spiracle, the sound producing structure, scale bar $1 \mathrm{~mm}$. Mandibles of a late instar caterpillar. (e) The mandibles of a fifth instar caterpillar in closed position, scale bar $0.5 \mathrm{~mm}$; (f) right mandible, inner face, scale bar $0.25 \mathrm{~mm}$; (g) left mandible, ventro-anterior view, scale bar $0.25 \mathrm{~mm}$; (h) close up of anterior edge of left mandible, scale bar $0.1 \mathrm{~mm}$. (i) List of defensive behaviours observed upon attack and when handled. (j) Histogram showing the relationship between sound production (S) and regurgitation $(R)$ during the first 5 pinches of an attack $(n=8)$. All images taken by Veronica Bura. 
a

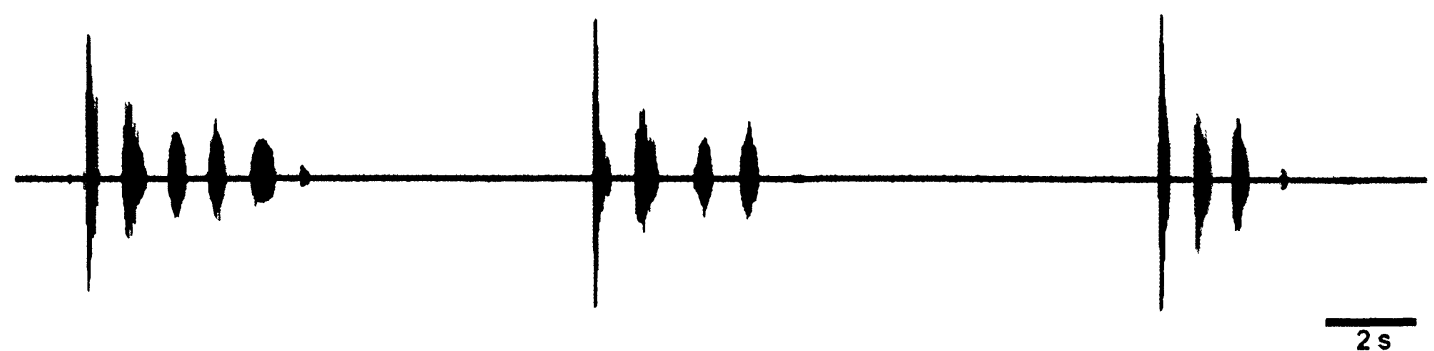

b
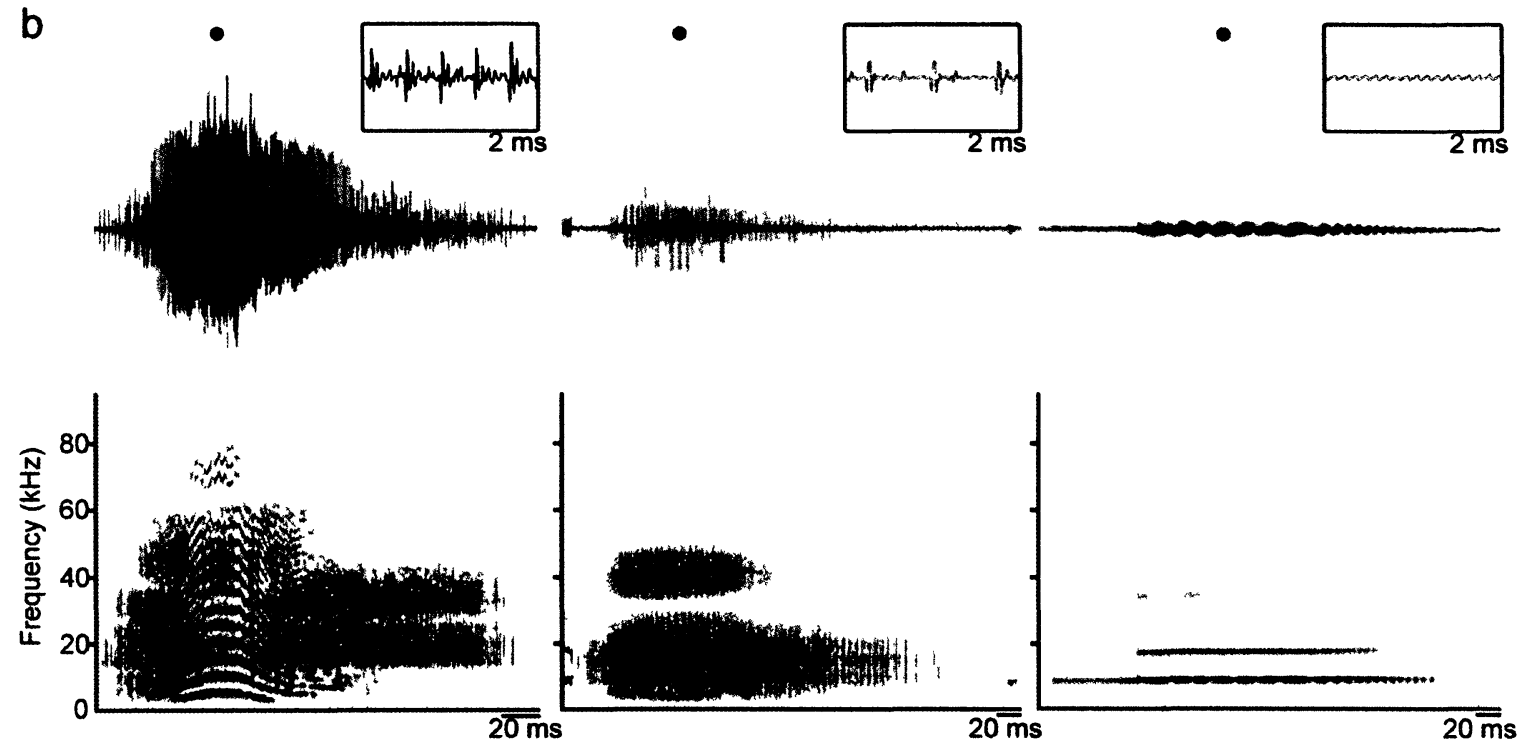

。
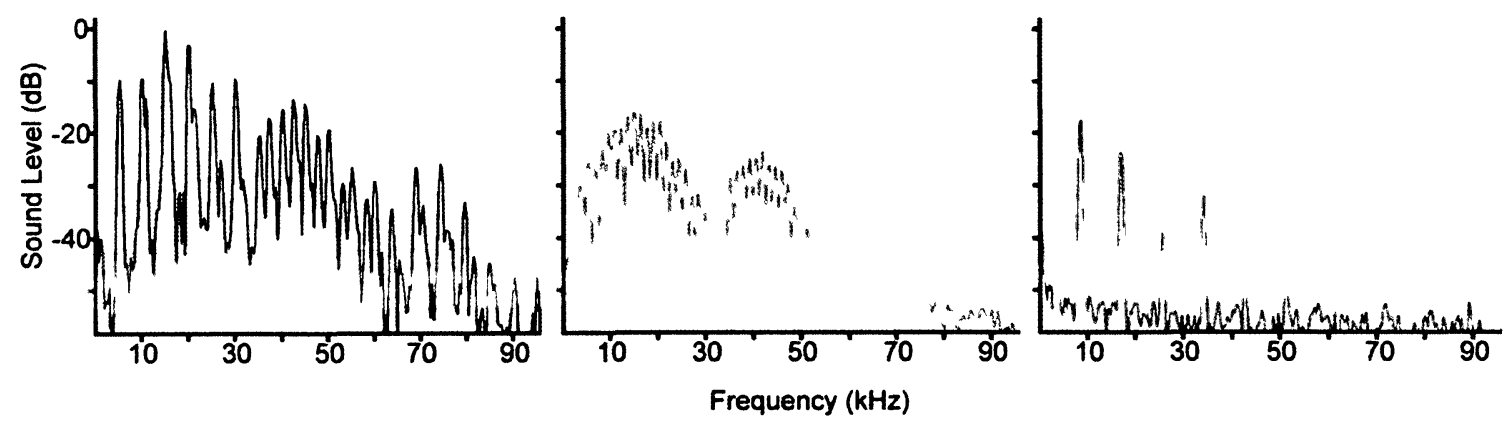

Figure 5.27 Amorpha juglandis sounds recorded from fifth instar larvae. (a) Oscillogram of three pulse trains following three consecutive attacks. Attack onset is marked with an arrow. (b) Oscillograms and corresponding spectrograms of time expanded pulses from the last train in (a), showing from left to right, Types 1,2 and 3. Insets represent an expanded time scale of the area represented by the black circle. (c) Power spectra of the 3 pulse types taken from the area represented by the black circle in (b). 
Mimas tiliae (Linnaeus), the Lime hawkmoth, Fig 5.28

The Lime hawkmoth is an old world moth found throughout Europe and western Asia (Pittaway 1993). Caterpillars of $M$. tiliae feed on plant species from 8 families, principally from Betulaceae (alder, Alnus sp.), Ulmaceae (elm, Ulmus sp.), Malvaceae (basswood, Tilia sp.) and Rosaceae (cherry, Prunus sp.) (Pittaway 1993).

Morphology: Larvae of this species occur in two morphs, green or bluish-white (Pittaway 1993). Final instar caterpillars were green (Fig 5.28 b), about $5.8 \mathrm{~cm}$ in length, had a triangular head with an area of $14.82 \mathrm{~mm}^{2}$ (Fig $5.28 \mathrm{c}$ ) and smooth mandibles with a slight blunt ridge on the inner face (Fig $5.28 \mathrm{~d}-\mathrm{g}$ ).

Behavioural Trials (Fig $5.28 \mathrm{~h}$ ): Lime hawkmoth caterpillars rest on the underside of leaves along the mid-vein (Pittaway 1993). Anterior attacks delivered to the head capsule caused the larvae to thrash away from the pinch and continue from side to side at a rate of about 2.75 flicks per second from the third proleg. One of the three specimens tested dropped from the leaf, releasing its legs as the pinch was delivered. No sound production was observed. I suspect that thrashing at high speeds is the main defence used by these caterpillars. 


\section{General Morphology}

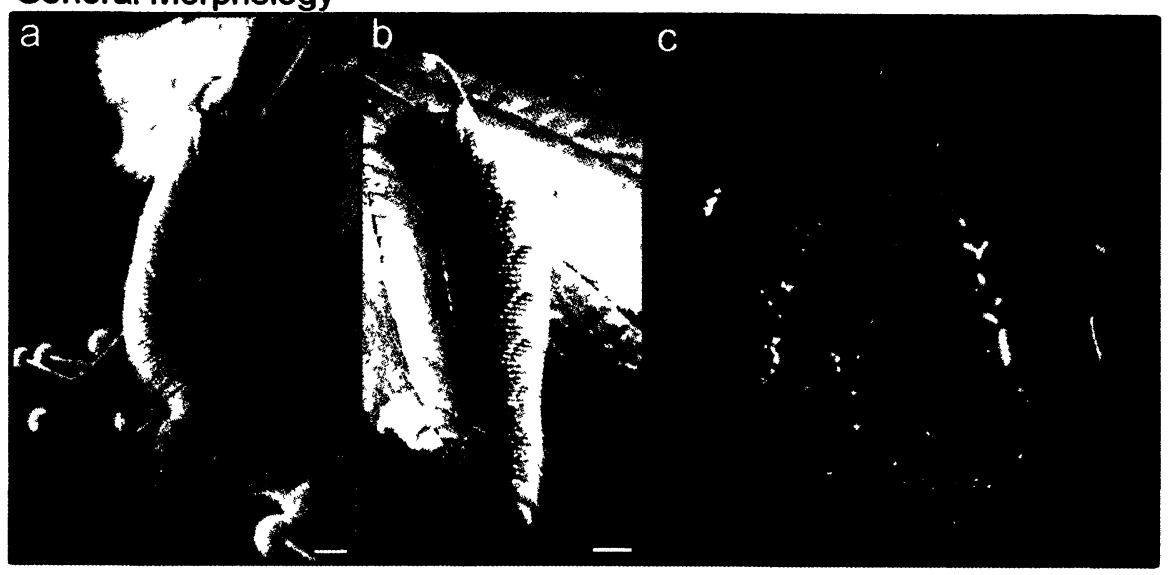

Mandibles

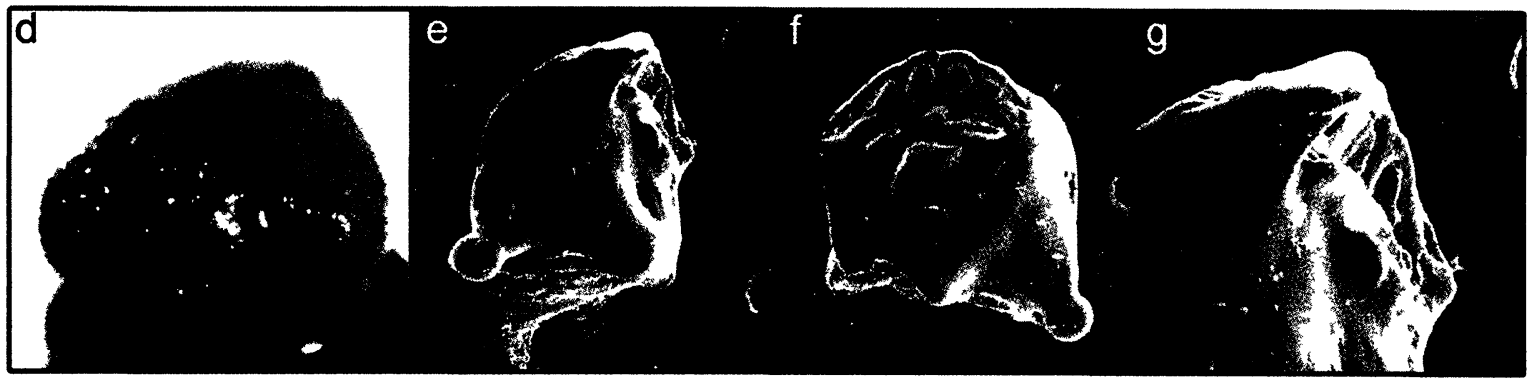

Defensive Behaviours

\begin{tabular}{|ll|}
\hline$\square$ Sound Production & $\square$ Biting \\
$\square$ Regurgitation & $\square$ Curling-in \\
$\square$ Chemical defense & $\square$ Curling up \\
$\square$ Thrashing & $\square$ Twitching \\
$\square$ Major thrashing & $\square$ Walk away \\
$\square$ Directed thrashing & $\square$ Dropping \\
\hline
\end{tabular}

Figure 5.28 Mimas tiliae, the Lime hawkmoth. General morphological features of a late instar caterpillar. (a) Dorso-lateral view of a late instar on its hostplant, anterior is down, scale bar 0.5 $\mathrm{cm}$. (b) Dorso-lateral view of a fifth instar caterpillar, anterior is down, scale bar $0.5 \mathrm{~cm}$. (c) Anterior view of the head capsule of a fifth instar, scale bar $0.5 \mathrm{~mm}$. Mandibles of a late instar caterpillar. (d) The mandibles of a late instar in closed position, scale bar $1 \mathrm{~mm}$; (e) right mandible, ventral-anterior view, scale bar $0.25 \mathrm{~mm}$; (f) left mandible, inner face, scale bar 0.25 $\mathrm{mm}$; (g) close up of anterior edge of right mandible, scale bar $0.1 \mathrm{~mm}$. (h) List of defensive behaviours observed upon attack and when handled. All images taken by Veronica Bura except (a) by J.C. Schou (http://www.biopix.com/Photo.asp?PhotoId=24911\&Photo=Lime-Hawk-moth(Mimas-tiliae) and (b) by Sarah Brown. 
Paonias excaecatus (JE Smith), the Blinded sphinx, Fig 5.29

The Blinded sphinx is a new world moth found much of North America (Wagner 2005, Tuttle 2007). Caterpillars of $P$. excaecatus feed on members of the Rosaceae, Salicaceae, Betulaceae and Ulmaceae families, and are considered to be generalized feeders (Wagner 2005, Tuttle 2007).

Morphology: The larvae of the Blinded sphinx can be highly variable, they are usually green, granular and may possess red splotches (Wagner 2005, Tuttle 2007). The brood that I reared did not possess any red splotching and was bright green (Fig $5.29 \mathrm{a}, \mathrm{b})$. Fifth instar larvae had an average length of $5.36 \mathrm{~cm}(\mathrm{n}=5)$, a triangular head with an area of $25.89 \mathrm{~mm}^{2}$ (Fig $5.29 \mathrm{c}$ ) and smooth mandibles with a blunt ridge on the inner face (Fig $5.29 \mathrm{~d}-\mathrm{g})$.

Behavioural Trials (Fig 5.29 h): Paonias excaecatus larvae rest on the underside of the leaf, gripping the mid-vein and petiole (Fig 5.29 a). When attacked posteriorly the larvae would curl-in and regurgitate. Two caterpillars also thrashed towards the site of attack. On anterior attacks the caterpillars would again curl-in, thrash away from the attack and regurgitate. No sound production was observed. Since regurgitation was observed in both sets of trials it may be the main defence for this species. However, it also appears to blend in well with its hostplant, indicating that crypsis may be the most important. 


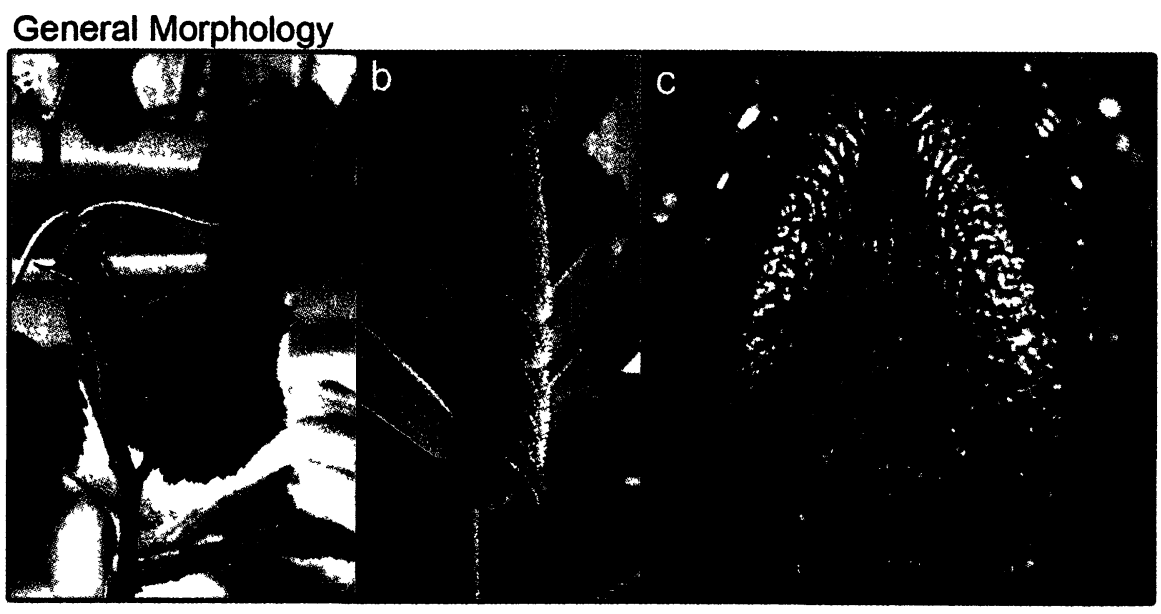

Mandibles

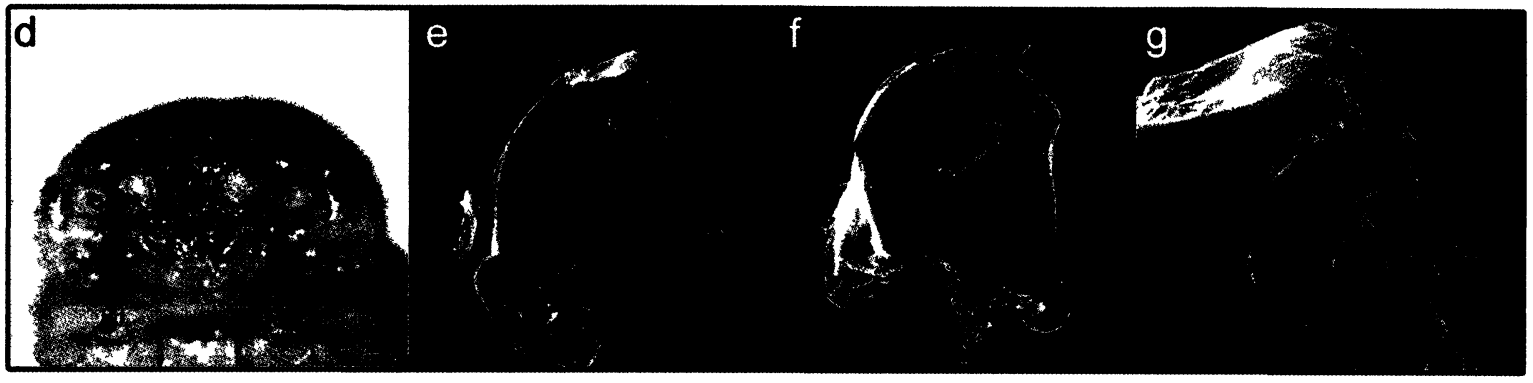

Defensive Behaviours

\begin{tabular}{|ll|}
\hline$\square$ Sound Production & $\square$ Biting \\
$\square$ Regurgitation & $\square$ Curling-in \\
$\square$ Chemical defense & $\square$ Curling up \\
$\square$ Thrashing & $\square$ Twitching \\
$\square$ Major thrashing & $\square$ Walk away \\
$\square$ Directed thrashing & $\square$ Dropping \\
\hline
\end{tabular}

Figure 5.29 Paonias excaecatus, the Blinded sphinx. General morphological features of a late instar caterpillar. (a) Lateral view of a late instar on its hostplant, anterior is down, scale bar $1 \mathrm{~cm}$. (b) Lateral view of a fifth instar caterpillar, scale bar $0.5 \mathrm{~cm}$. (c) Anterior view of the head capsule of a fifth instar, scale bar $0.5 \mathrm{~mm}$. Mandibles of a late instar caterpillar. (d) The mandibles of a late instar in closed position, scale bar $0.5 \mathrm{~mm}$; (e) right mandible, ventral-anterior view, scale bar $0.25 \mathrm{~mm}$; (f) left mandible, inner face, scale bar $0.25 \mathrm{~mm}$; (g) close up of anterior edge of right mandible, scale bar $0.1 \mathrm{~mm}$. (h) List of defensive behaviours observed upon attack and when handled. All images taken by Veronica Bura. 


\section{Paonias myops (JE Smith), the Small-Eyed sphinx, Fig 5.30}

The Small-Eyed sphinx is a new world moth found throughout much of North America (Wagner 2005, Tuttle 2007). Larvae of this species feed on members of the Rosaceae family, principally cherries (Prunus sp.) (Wagner 2005, Tuttle 2007). Morphology: Late instar caterpillars are green with several red splotches (Fig $5.30 \mathrm{a}, \mathrm{b}$ ), said to resemble the spots on mature cherry trees (Tuttle 2007). Larvae were approximately $4.5 \mathrm{~cm}$ long in their fifth instar $(\mathrm{n}=5)$, had a triangular head with an area of $16.43 \mathrm{~mm}^{2}$ (Fig $5.30 \mathrm{c}$ ) and had smooth mandibles (Fig $5.30 \mathrm{~d}-\mathrm{g}$ ).

Behavioural Trials (Fig 5.30 h): Caterpillars of $P$. myops rest along the mid-vein on the underside of the leaf and feed mainly at night (Wagner 2005, Tuttle 2007). To remove evidence of their presence and remain cryptic, these larvae eliminate partially eaten leaves by clipping them at their base (Wagner 2005). Both anterior and posterior attacks caused the larvae to curl-in slightly and regurgitate. Anterior attacks also caused the larvae to back up slowly along the mid-vein a few steps, but the larvae never left the area completely. These caterpillars would also bite and regurgitate when handled. No sound production was observed. As with its congener, the main defence for this species is likely crypsis or regurgitation. Since the red splotches are said to resemble the spots on its hostplant, I would lean towards crypsis in this case. 

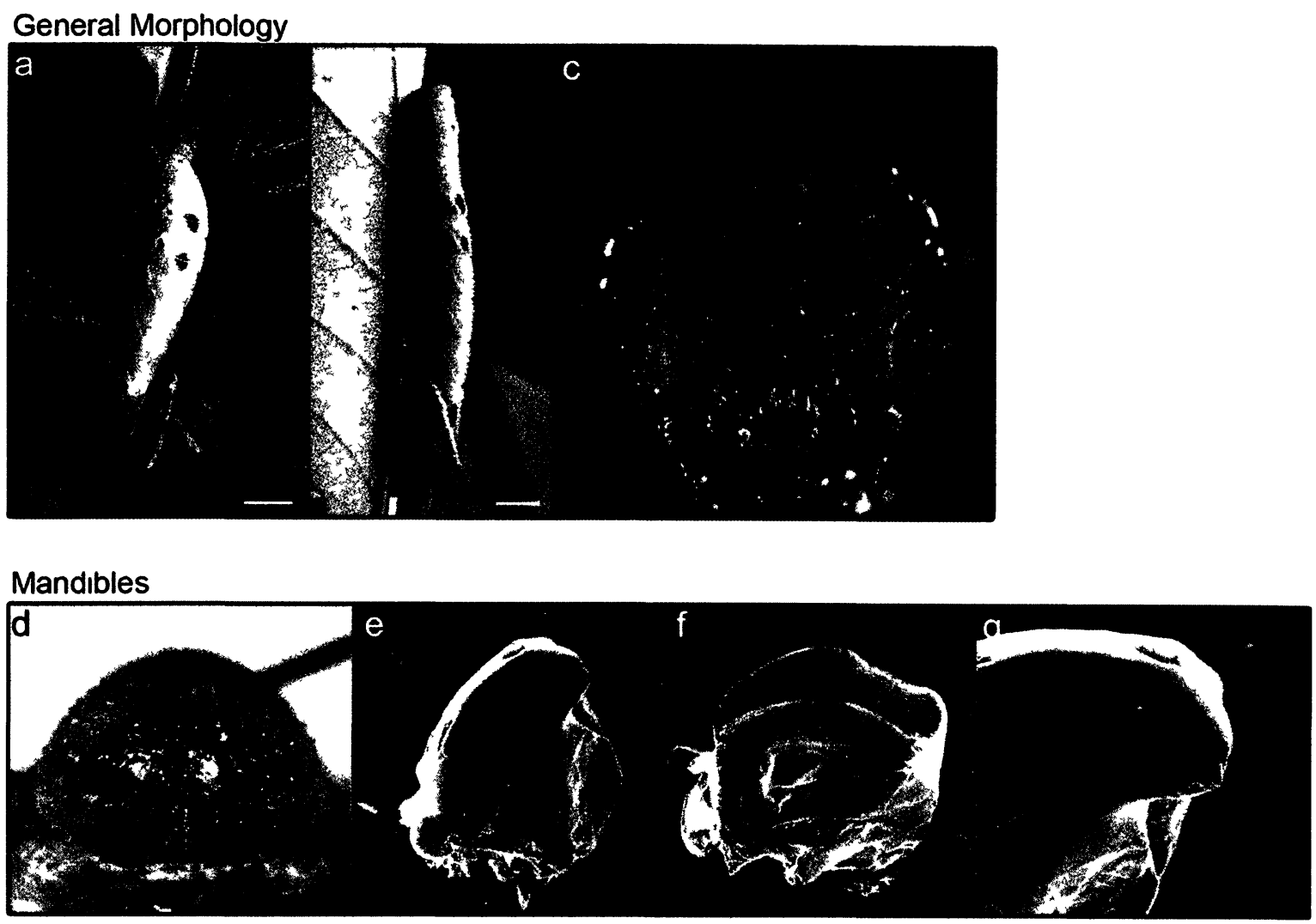

\section{Defensive Behaviours}

\begin{tabular}{|ll|}
\hline$\square$ Sound Production & Biting \\
$\square$ Regurgitation & Curling-In \\
$\square$ Chemical defense & $\square$ Curling up \\
$\square$ Thrashing & $\square$ Twitching \\
$\square$ Major thrashing & $\square$ Walk away \\
$\square$ Directed thrashing & $\square$ Dropping \\
\hline
\end{tabular}

Figure 5.30 Paonias myops, the Small-Eyed sphinx. General morphological features of a late instar caterpillar. (a) Lateral view of a late instar on its hostplant, scale bar $0.75 \mathrm{~cm}$. (b) Lateral view of a fifth instar caterpillar, scale bar $0.5 \mathrm{~cm}$. (c) Anterior view of the head capsule of a fifth instar, scale bar $0.5 \mathrm{~mm}$. Mandibles of a late instar caterpillar. (d) The mandibles of a late instar in closed position, scale bar $0.5 \mathrm{~mm}$; (e) right mandible, ventral-anterior view, scale bar $0.25 \mathrm{~mm}$; (f) left mandible, inner face, scale bar $0.25 \mathrm{~mm}$; (g) close up of anterior edge of right mandible, scale bar $0.1 \mathrm{~mm}$. (h) List of defensive behaviours observed upon attack and when handled. All images taken by Veronica Bura, except (a) by L. Massman (http://bugguide.net/node/view/ 338342/bgimage). 
Smerinthus cerisyi Kirby, the One-Eyed sphinx, Fig 5.31

The One-Eyed sphinx is a new world moth found throughout much of Canada and northern and western United States (Wagner 2005, Tuttle 2007). Larvae of this species feed exclusively on members of the Saliaceae (willow) family, principally poplar and willow (Wagner 2005, Tuttle 2007).

Morphology: One-Eyed sphinx caterpillars can be highly variable, ranging from pale yellow to blue-green (Fig 5.31 a,b, Wagner 2005). Tested larvae were lime green (Fig $5.31 \mathrm{~b}$ ), $5.7 \mathrm{~cm}$ in length, had a triangular head with an area of $21.32 \mathrm{~mm}^{2}$ (Fig $5.31 \mathrm{c}$ ) and smooth mandibles with a blunt ridge on the inner face (Fig $5.31 \mathrm{~d}-\mathrm{g}$ ).

Behavioural Trials (Fig 5.31 h): Late instar caterpillars rest on the underside of the leaf along the mid-vein or petiole (Fig $5.31 \mathrm{~b}$ ). When attacked anteriorly $S$. cerisyi caterpillars would curl-in and thrash away from the pinch and subsequently regurgitate. No sound production was observed. I suggest that either crypsis or regurgitation is the main defence for these green caterpillars. 


\section{General Morphology}

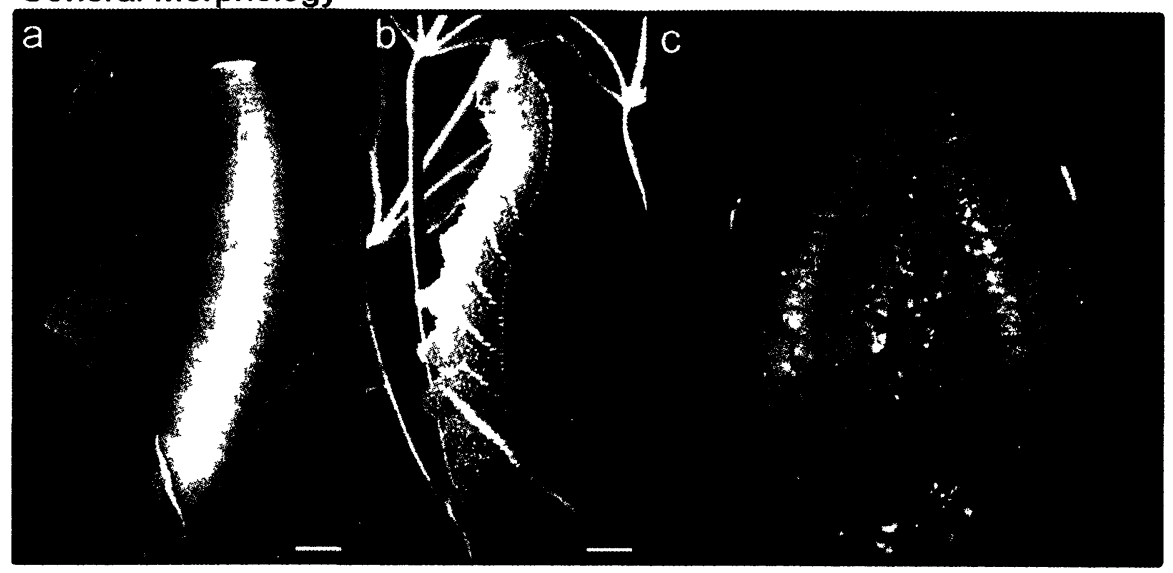

Mandibles

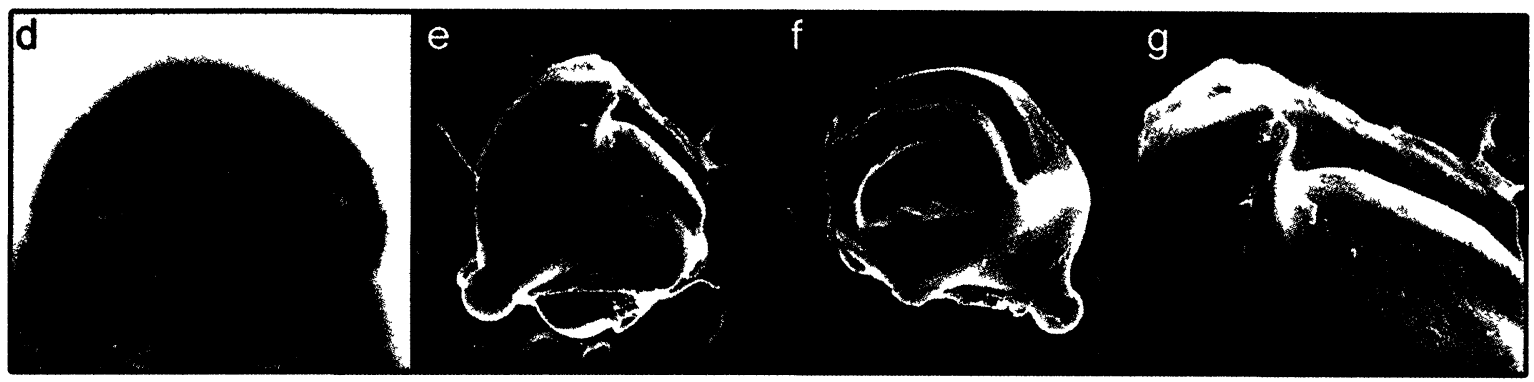

Defensive Behaviours

\begin{tabular}{|ll|}
\hline h Sound Production & $\square$ Biting \\
$\square$ Regurgitation & $\square$ Curling-in \\
$\square$ Chemical defense & $\square$ Curling up \\
$\square$ Thrashing & $\square$ Twitching \\
$\square$ Major thrashing & $\square$ Walk away \\
$\square$ Directed thrashing & $\square$ Dropping \\
\hline
\end{tabular}

Figure 5.31 Smerinthus cerisyi, the One-Eyed sphinx. General morphological features of a late instar caterpillar. (a) Dorsal view of a late instar on its hostplant, scale bar $0.5 \mathrm{~cm}$. (b) Lateral view of a fifth instar caterpillar, scale bar $0.5 \mathrm{~cm}$. (c) Anterior view of the head capsule of a fifth instar, scale bar $0.5 \mathrm{~mm}$. Mandibles of a late instar caterpillar. (d) The mandibles of a late instar in closed position, scale bar $0.5 \mathrm{~mm}$; (e) right mandible, ventral-anterior view, scale bar $0.25 \mathrm{~mm}$; (f) left mandible, inner face, scale bar $0.25 \mathrm{~mm}$; (g) close up of anterior edge of right mandible, scale bar $0.1 \mathrm{~mm}$. (h) List of defensive behaviours observed upon attack and when handled. All images taken by Veronica Bura, except (a) by Bill Oehkle (http://www.silkmoths.bizland.com/ scerisyi.htm) and (b) by Sarah Brown. 
Smerinthus jamaicensus (Drury), the Twin-Spotted sphinx, Fig 5.32

The Twin-Spotted sphinx is a new world moth found throughout much of North America (Wagner 2005, Tuttle 2007). Larvae feed on members of the Salicaceae and Ulmaceae, principally on willows (Salix sp.) (Wagner 2005, Tuttle 2007).

Morphology: Caterpillars of S. jamaicensus can be blue-green to lime green and have a blue horn (Wagner 2005, Tuttle 2007). Fifth instar larvae were blue-green (Fig 5.32 a,b), about $5 \mathrm{~cm}$ long, had a triangular head with an area of $21.42 \mathrm{~mm}^{2}$ (Fig $5.32 \mathrm{c}$ ) and smooth mandibles with a slight blunt ridge on the inner face (Fig $5.32 \mathrm{~d}-\mathrm{g}$ ).

Behavioural Trials (Fig $5.32 \mathrm{~h}$ ): Larvae typically rest on the underside of leaves (Wagner 2005). When attacked anteriorly caterpillars would thrash away from the pinch, curl-in and subsequently regurgitate. No sound production was observed, in contrast to that reported by Sanborn (1868). As for the other green caterpillars in this subfamily, I suspect that crypsis or regurgitation is the main defence employed by this species. 


\section{General Morphology}

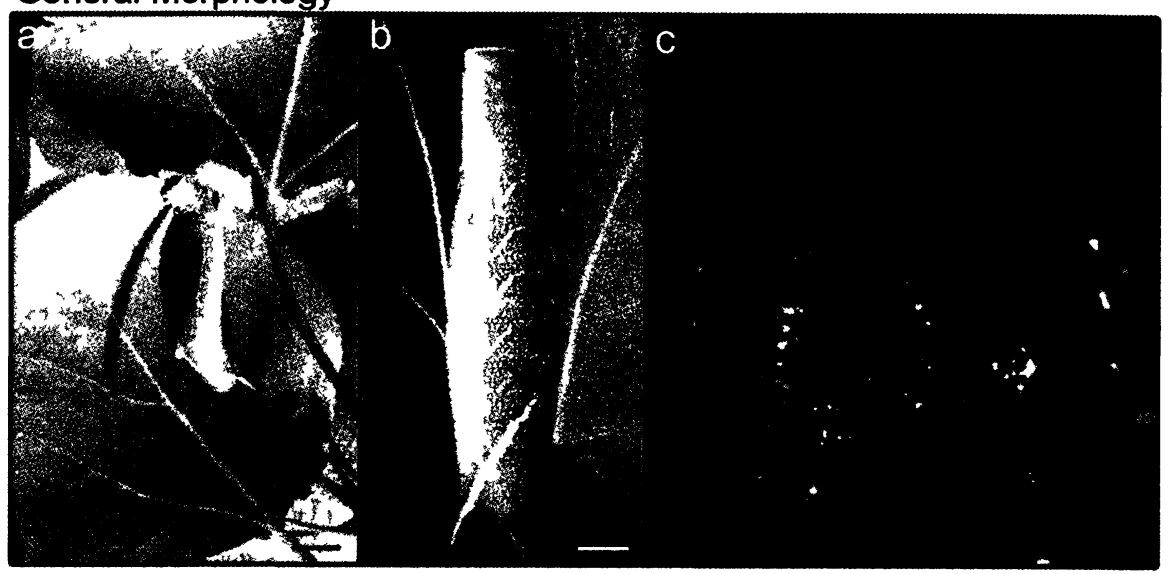

Mandibles

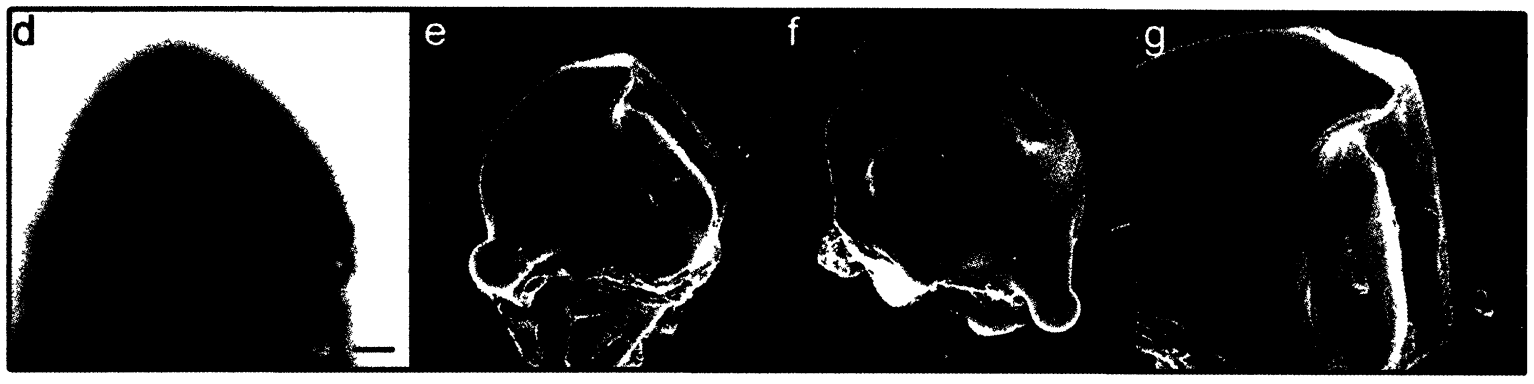

Defensive Behaviours

\begin{tabular}{|ll|}
\hline h Sound Production & $\square$ Biting \\
Regurgitation & $\square$ Curling-in \\
$\square$ Chemical defense & $\square$ Curling up \\
$\square$ Thrashing & $\square$ Twitching \\
$\square$ Major thrashing & $\square$ Walk away \\
$\square$ Directed thrashing & $\square$ Dropping \\
\hline
\end{tabular}

Figure 5.32 Smerinthus jamaicensus, the Twin-Spotted sphinx. General morphological features of a late instar caterpillar. (a) Dorsal view of a late instar on its hostplant, scale bar $1 \mathrm{~cm}$. (b) Lateral view of a fifth instar caterpillar, scale bar $0.5 \mathrm{~cm}$. (c) Anterior view of the head capsule of a fifth instar, scale bar $0.5 \mathrm{~mm}$. Mandibles of a late instar caterpillar. (d) The mandibles of a late instar in closed position, scale bar $0.5 \mathrm{~mm}$; (e) right mandible, ventral-anterior view, scale bar $0.25 \mathrm{~mm}$; (f) left mandible, inner face, scale bar $0.25 \mathrm{~mm}$; (g) close up of anterior edge of right mandible, scale bar $0.1 \mathrm{~mm}$. (h) List of defensive behaviours observed upon attack and when handled. All images taken by Veronica Bura, except (a) and (b) by Antoine Hnain. 


\section{SUBFAMILY: SPHINGINAE}

Crypsis is the primary stradegy used by the majority of species in this group, many species are countershaded and occur in different colour morphs (Kitching and Cadiou 2000).

Acherontia atropos (Linnaeus), the Death's Head Hawkmoth, Fig 5.33

The Death's Head Hawkmoth is an old world moth found along the Mediterranean Sea in northern Africa, the Middle East and southern Europe, occasionally being found in central and northern Europe (Pittaway 1993). Larvae of this species feed principally on members of the nightshade or potato family (Solanaceae), but also Verbenaceae (verbena family), Oleaceae (olive family), Beta sp. (beets), Nerium sp. (oleander), Buddleja sp., and others (Pittaway 1993).

Morphology: Larvae of $A$. atropos can be green, brown or yellow in their final instar, green being the most common (Pittaway 1993). At full size, the final instar of this species is capable of achieving a length of $12-13 \mathrm{~cm}$ (Pittaway 1993).

Behaviour and Acoustics: Late instar caterpillars are highly inactive and rest by hanging underneath the leaves of hostplant, using the stem or petiole as an anchor (Pittaway 1993). I was unable to obtain any video footage of this species and therefore consider this data to be incomplete. I can report on the characteristics of the sounds as I was able to get recordings. I suspect that these caterpillars would directed thrash, thrash from side to side and regurgitate in addition to the noted behaviours of clicking and biting (Pittaway 1993). Sound characteristics (Table 5.3): The acoustic signals consisted of trains of clicks (Fig $5.34 \mathrm{a}, \mathrm{b})$ that lasted, on average, $3025 \pm 1514 \mathrm{~ms}$ and contained $4-15$ clicks ( $\mathrm{n}=10$ trains from 3 animals). Individual clicks had a mean duration of $13.70 \pm 3.09 \mathrm{~ms}$ and contained 
$1-5$ components $(\mathrm{n}=90$ clicks). Clicks were broadband (mean Q3 $=3.51 \pm 1.28, \mathrm{Q} 10=$ $1.28 \pm 0.59, \mathrm{n}=20$ clicks from 4 animals) and had a mean dominant frequency of $23.33 \pm$ $2.97 \mathrm{kHz}$ (Fig $5.34 \mathrm{c})$.

Mechanism: Though unconfirmed experimentally, the suspected mechanism of clicking is through the use of the mandibles (Pittaway 1993, Brown et al. 2007 and references therein). 


\section{General Morphology}

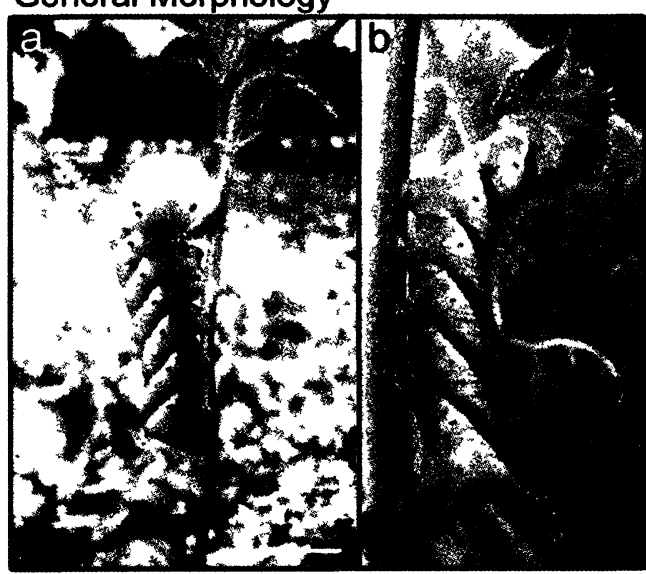

Defensive Behaviours

\begin{tabular}{|ll|}
\hline $\mathbb{E}$ Sound Production & Biting \\
$\mathbb{E}$ Regurgitation & $\square$ Curling-in \\
$\square$ Chemical defense & $\square$ Curling up \\
$\mathbb{E}$ Thrashing & $\square$ Twitching \\
$\square$ Major thrashing & $\square$ Walk away \\
$\mathbb{E}$ Directed thrashing & $\square$ Dropping \\
\hline
\end{tabular}

Figure 5.33 Acherontia atropos, the Death's Head hawkmoth. General morphological features of a late instar caterpillar. (a) Dorsal view of the caterpillar on its hostplant, scale bar $1 \mathrm{~cm}$. (b) Lateral view of a fifth instar caterpillar, scale bar $0.5 \mathrm{~cm}$. (c) List of defensive behaviours noted in the literature. Suspected behaviours are indicated by the open check marks and have not been confirmed. Image in (a) by FranciscoGC (http://static.panoramio.com/photos/original/ 12098143.jpg), (b) by Jayne Yack. 
a
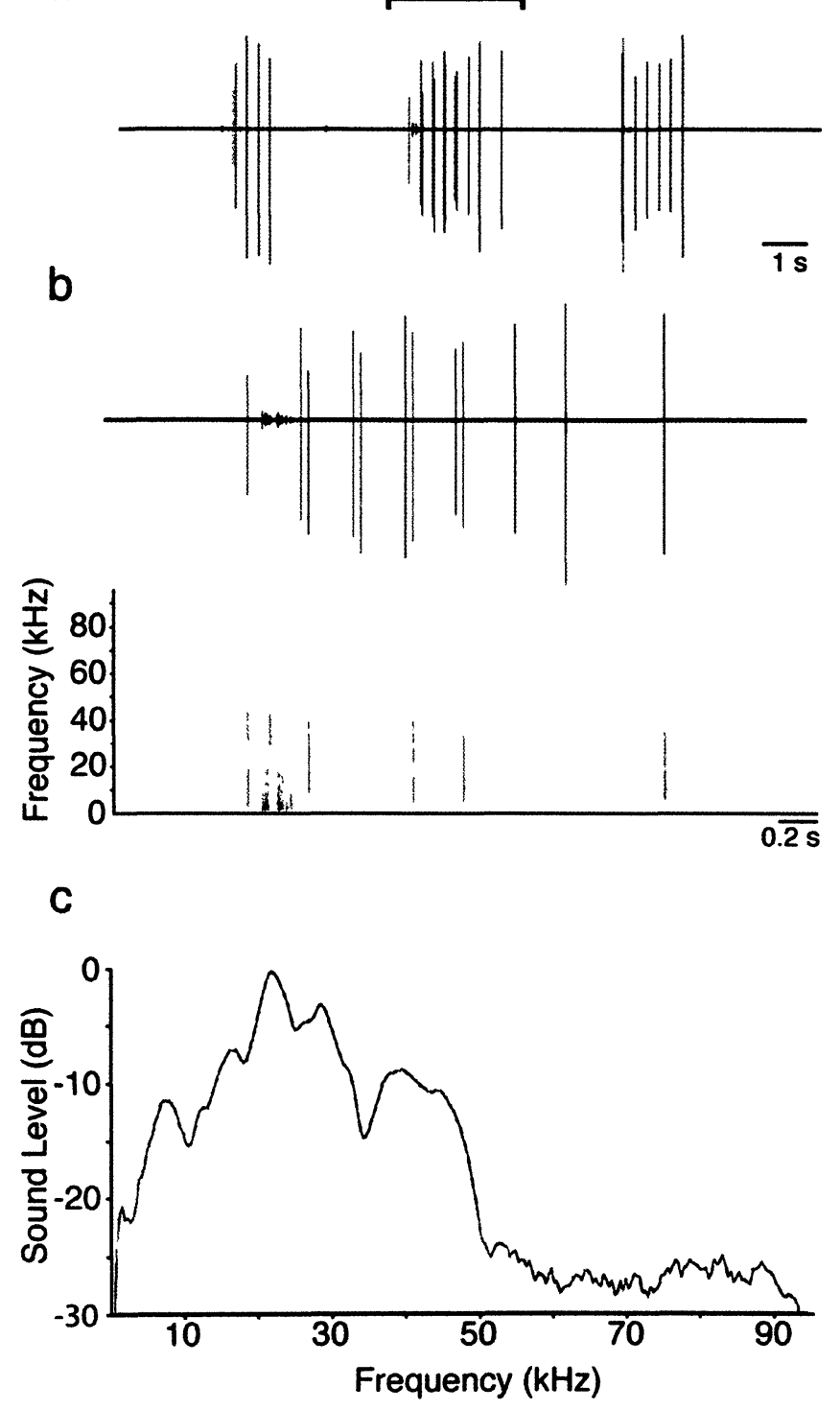

Figure 5.34 Sounds produced by late instar $\boldsymbol{A}$. atropos. (a) Oscillogram of clicks following attack. (b) Time expansion of bracket in (a) showing the multiple components of each click. The accompanying spectrogram shows the frequency distribution of each click. (c) Power spectrum taken from one individual. 
Ceratomia undulosa (Walker), the Waved sphinx, Fig 5.35

The Waved sphinx is a new world moth found throughout eastern and central North America (Wagner 2005, Tuttle 2007). Caterpillars of this species feed mainly on members of the olive family (Oleaceae), including ash (Fraxinus sp.), privet (Ligustrum sp.) and lilac (Syringa sp.) (Wagner 2005, Tuttle 2007).

Morphology: Larvae of the Waved sphinx can be highly variable (Tuttle 2007). In the brood that I reared, there were three different variations though green was always the principle colour. One was very pale with primarily white accents (Fig 5.35 a), another was a darker green with a pink horn and a bit of pink along its lateral stripes (Fig 5.35 b) and the third was the same darker green with a pink horn and head capsule and large pink splotches along its lateral stripes. All varieties had the characteristic black stipulations on the anal plate and claspers (Wagner 2005). Fifth instar caterpillars were, on average, 5.7 $\mathrm{cm}$ in length $(\mathrm{n}=5)$ at the time of experimentation, had an oval head with an area of $26.78 \mathrm{~mm}^{2}$ (Fig $5.35 \mathrm{c}$ ) and ridged and serrated mandibles (Fig $5.35 \mathrm{~d}-\mathrm{g}$ ).

Behavioural trials (Fig 5.35 h): Ceratomia undulosa caterpillars rested affixed to the midrib on the underside of the leaves of their hostplant. When attacked anteriorly the larvae would thrash away from the pinch, curl-in and thrash from side to side. Posterior attacks would cause the caterpillars to curl-in and subsequently regurgitate. Small side to side thrashing and twitching were also observed on these attacks. No sound production was observed. I suspect the main defence used by this species is crypsis. 


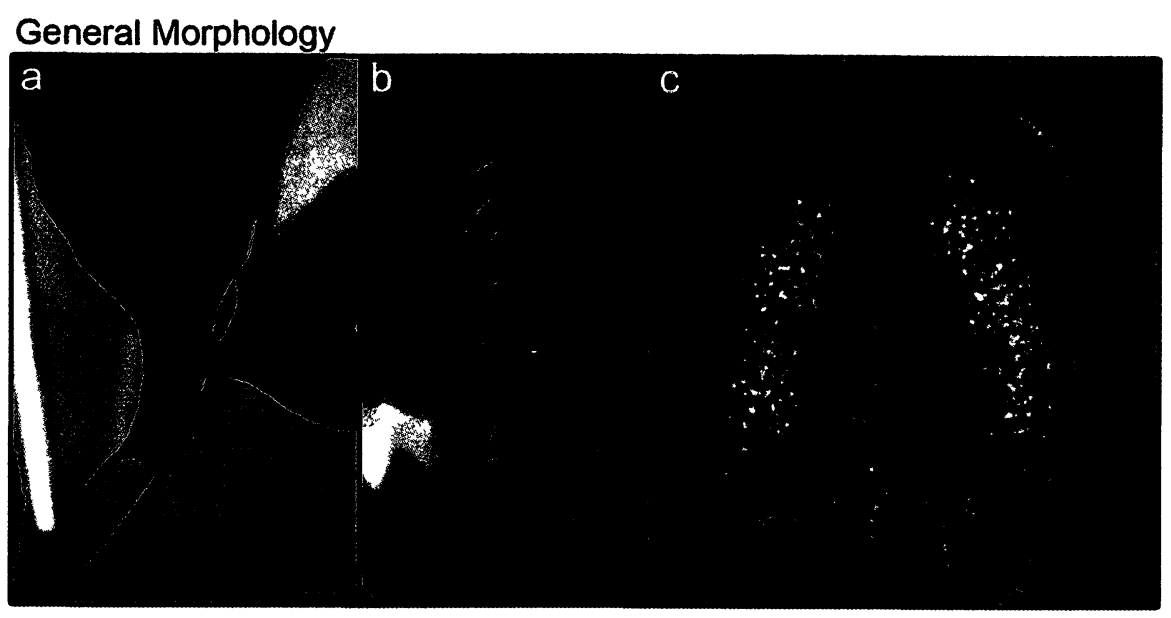

\section{Mandibles}

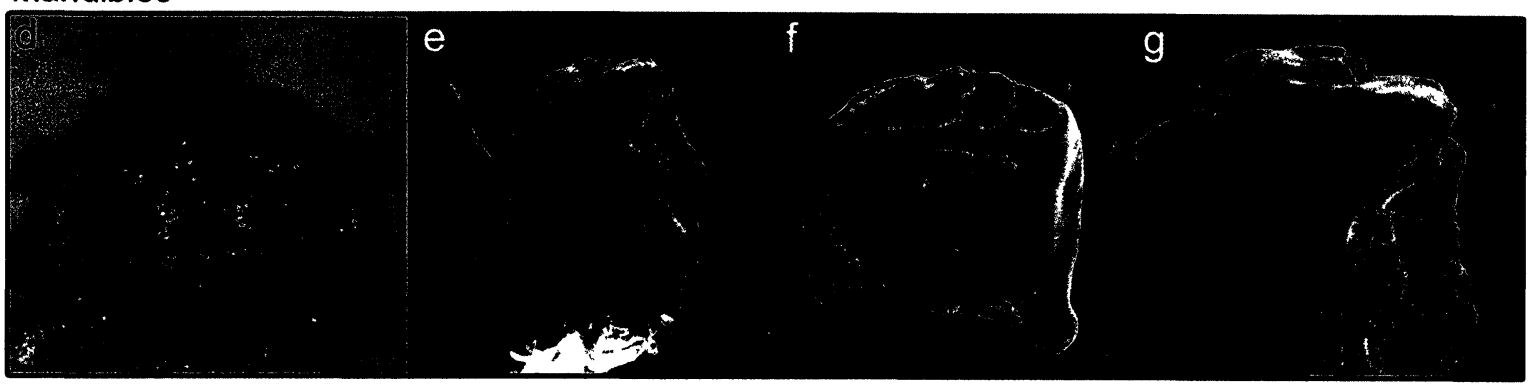

Defensive Behaviours

\begin{tabular}{|ll|}
\hline h Sound Production & $\square$ Biting \\
$\square$ Regurgitation & $\square$ Curling-in \\
$\square$ Chemical defense & $\square$ Curling up \\
$\square$ Thrashing & $\square$ Twitching \\
$\square$ Major thrashing & $\square$ Walk away \\
$\square$ Directed thrashing & $\square$ Dropping \\
\hline
\end{tabular}

Figure 5.35 Ceratomia undulosa, the Waved sphinx. General morphological features of a late instar caterpillar. (a) Lateral view of a fifth instar larva on ash leaves, scale bar $1 \mathrm{~cm}$. (b) Lateral view of a fifth instar caterpillar, scale bar $5 \mathrm{~mm}$. (c) Anterior view of the head capsule of a fifth instar, scale bar $0.5 \mathrm{~mm}$. Mandibles of a late instar caterpillar. (d) The mandibles of a late instar in closed position, scale bar $0.5 \mathrm{~mm}$; (e) right mandible, ventral-anterior view, scale bar $0.25 \mathrm{~mm}$; (f) left mandible, inner face, scale bar $0.25 \mathrm{~mm}$; (g) close up of anterior edge of left mandible, scale bar $0.1 \mathrm{~mm}$. (h) List of defensive behaviours observed upon attack and when handled. All images taken by Veronica Bura. 


\section{Manduca albiplaga (Walker), the White-plaqued sphinx, Fig 5.36}

Manduca albiplaga is a new world moth found throughout Mexico, Central America and South America (Oehkle 2009, Savela 2009). Caterpillars feed on members of the Boraginaceae (Borage or Forget-me-not) and Annonaceae (custard apple) families (Janzen and Hallwachs 2009).

Morphology: The final instar caterpillar of the White-plaqued sphinx was a bright lime green dorsally and a paler green ventrally (Fig $5.36 \mathrm{a}, \mathrm{b}$ ). It had a pink horn and white and black lateral stripes on each segment. This specimen was $8.3 \mathrm{~cm}$ in length at the time of experimentation.

Behavioural Trials (Fig 5.36 c): The Manduca albiplaga larva was resting on the midvein on the underside of the leaf prior to experimentation (Fig 5.36 a). It attached itself using its claspers and prolegs, letting the anterior portion of its body hang. When attacked anteriorly the caterpillar thrashed away from the pinch and subsequently began thrashing from side to side at a rate of approximately 2.38 flicks per second from proleg 4. Posterior attacks caused the larva to spew copious amounts of regurgitant towards the attacking forceps, and subsequently thrash from side to side. No sound production was observed. I suggest that major thrashing combined with regurgitation is the main defence for this species. 
General Morphology

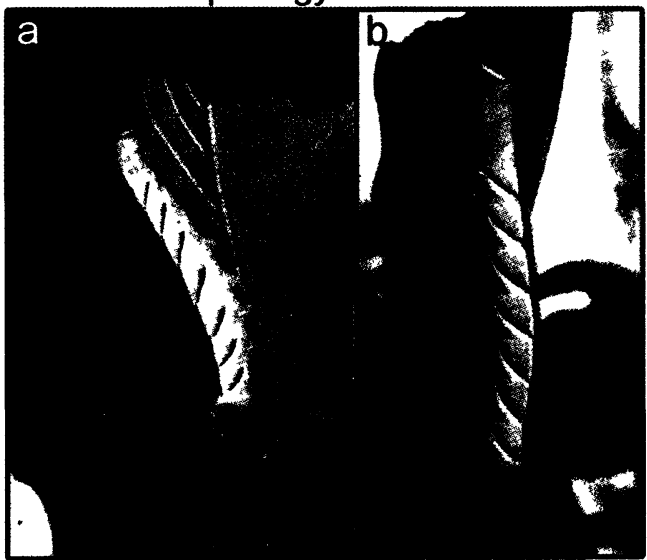

Defensive Behaviours

\begin{tabular}{|ll|}
\hline$\square$ Sound Production & $\square$ Biting \\
$\square$ Regurgitation & $\square$ Curling-in \\
$\square$ Chemical defense & $\square$ Curling up \\
$\square$ Thrashing & $\square$ Twitching \\
$\square$ Major thrashing & $\square$ Walk away \\
$\square$ Directed thrashing & $\square$ Dropping \\
\hline
\end{tabular}

Figure 5.36 Manduca albiplaga. General morphological features of a late instar caterpillar. (a) Dorsal view of the caterpillar on its hostplant, scale bar $1 \mathrm{~cm}$. (b) Lateral view of a fifth instar caterpillar, scale bar $0.5 \mathrm{~cm}$. (c) List of defensive behaviours observed upon attack and when handled. All images taken by Alan Fleming. 
Manduca florestan (Stoll), Fig 5.37

Manduca florestan is a new world moth found from southern Arizona and New Mexico through Central America to northern South America (Oehkle 2009, Savela 2009). Larvae feed on species from at least 9 different plant families including Fabaceae, Oleaceae and Solanaceae (Tuttle 2007, Janzen and Hallwachs 2009). Morphology: The fifth instar M. florestan that I tested was green and covered in white stripes that ran from its dorsal side laterally and anteriorly towards the ventral side (Fig $5.37 \mathrm{a}$ ). This individual was $7.8 \mathrm{~cm}$ in length at the time of experimentation. Behavioural Trials (Fig 5.37 c): This caterpillar appears to rest on the underside of the leaf along the mid-vein, holding on using its claspers and prolegs and letting the anterior portion of its body hang (Fig $5.37 \mathrm{~b}$ ). Both anterior and posterior attacks caused this caterpillar to thrash from side to side in a wide arc at a rate of approximately 2 flicks per second from either proleg 3 or 4 . No sound production was observed. 


\section{General Morphology}

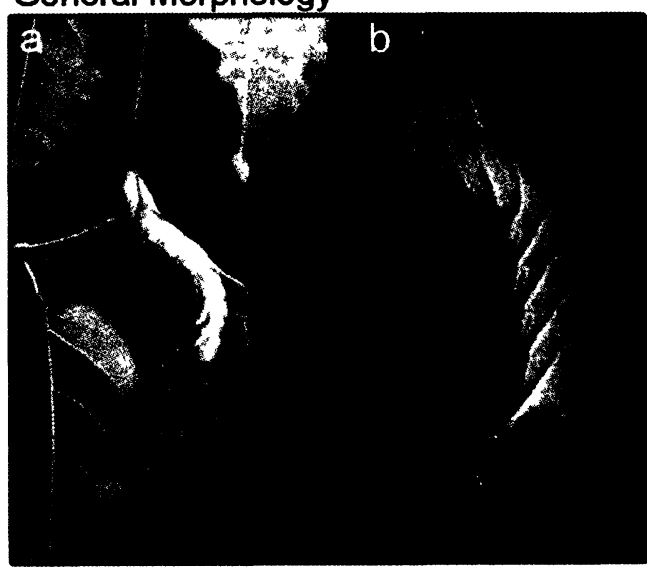

\section{Defensive Behaviours}

\begin{tabular}{|ll|}
\hline$\square$ Sound Production & $\square$ Biting \\
$\square$ Regurgitation & $\square$ Curling-in \\
$\square$ Chemical defense & $\square$ Curling up \\
$\square$ Thrashing & $\square$ Twitching \\
$\square$ Major thrashing & $\square$ Walk away \\
$\square$ Directed thrashing & $\square$ Dropping \\
\hline
\end{tabular}

Figure 5.37 Manduca floristan. General morphological features of a late instar caterpillar. (a) Dorsal view of the caterpillar on its hostplant, anterior is down, scale bar $1 \mathrm{~cm}$. (b) Lateral view of a fifth instar caterpillar, scale bar $0.5 \mathrm{~cm}$. (c) List of defensive behaviours observed upon attack and when handled Image in (a) by Jayne Yack, (b) by Dan Janzen. 
Manduca pellenia (Herrich-Schaffer), Fig 5.38

Manduca pellenia is a new world moth found from northern Mexico, through Central America to northern South America (Oehkle 2009, Savela 2009). This species feeds exclusively on members of the Solanaceae family (Janzen and Hallwachs 2009). Morphology: The final instar M. pellenia caterpillar is green with white and lime-green stripes running laterally and anteriorly from dorsal to ventral (Fig $5.38 \mathrm{a}, \mathrm{b}$ ). It has a limegreen horn and yellow stipulations on the dorsal side of its thorax. My specimen was approximately $8.8 \mathrm{~cm}$ in length, had a rounded head capsule with an area of about 38 $\mathrm{mm}^{2}$ (Fig $5.38 \mathrm{c}$ ) and ridged and serrated mandibles (Fig $5.38 \mathrm{~d}-\mathrm{g}$ ).

Behavioural Trials (Fig $5.38 \mathrm{~h}$ ): Manduca pellenia larvae rest on the underside of the leaf along the mid-vein, holding on with their prolegs and claspers and letting the anterior portion of its body hang (Fig $5.38 \mathrm{~b}$ ). When attacked anteriorly the fifth instar caterpillar regurgitated and began thrashing from side to side at a rate of about 2.17 flicks per second from proleg 3 or 4 . Posterior attacks elicited similar behaviours with the addition of some quiet clicking. The larva responded to being repositioned by biting. Acoustics (Fig 5.39): Caterpillars click in response to attack.

Sound characteristics: The acoustic signals produced by M. pellenia consist of trains of quiet clicks (Fig 5.39 a,b). Trains were on average $2269 \pm 1339 \mathrm{~ms}$ in duration, containing 5-8 clicks ( $\mathrm{n}=3$ trains from 1 animal). Clicks were typically $2.63 \pm 8.09 \mathrm{~ms}$ in duration and contained 1-2 components $(\mathrm{n}=19$ clicks). Sounds were broadband (mean $\mathrm{Q} 3=2.62 \pm 1.47, \mathrm{Q} 10=0.88 \pm 0.64, \mathrm{n}=5$ clicks from 1 animal) and had a mean dominant frequency of $17.55 \pm 17.03 \mathrm{kHz}$ (Fig $5.39 \mathrm{c}$ ). 
Mechanism: Although I did not investigate this directly, based on the similarity of these clicks to those of other species I would suggest that they are produced using the mandibles. Likely, ridges on opposing mandibles come into contact as the mandibles are closed to produce a click, though verification is required.

Behaviours associated with sound production and proposed function: Clicks were only noticeable when the caterpillar was attacked posteriorly, and even then they were difficult to hear. Clicks occurred while the caterpillar remained motionless in between successive bouts of thrashing. During the first five pinches of an attack, the production of sound followed the appearance of regurgitation (Fig 5.38 i). However, sound production was not observed on the anterior attack (the fifth pinch was a posterior attack), which was preformed first, so it is difficult to say if regurgitation occurred before or after sound production. Considering only the posterior attack it appeared as though regurgitation occurred following the first appearance of sound production, suggesting a warning function for the sounds, but this would need to be verified with additional trials. 


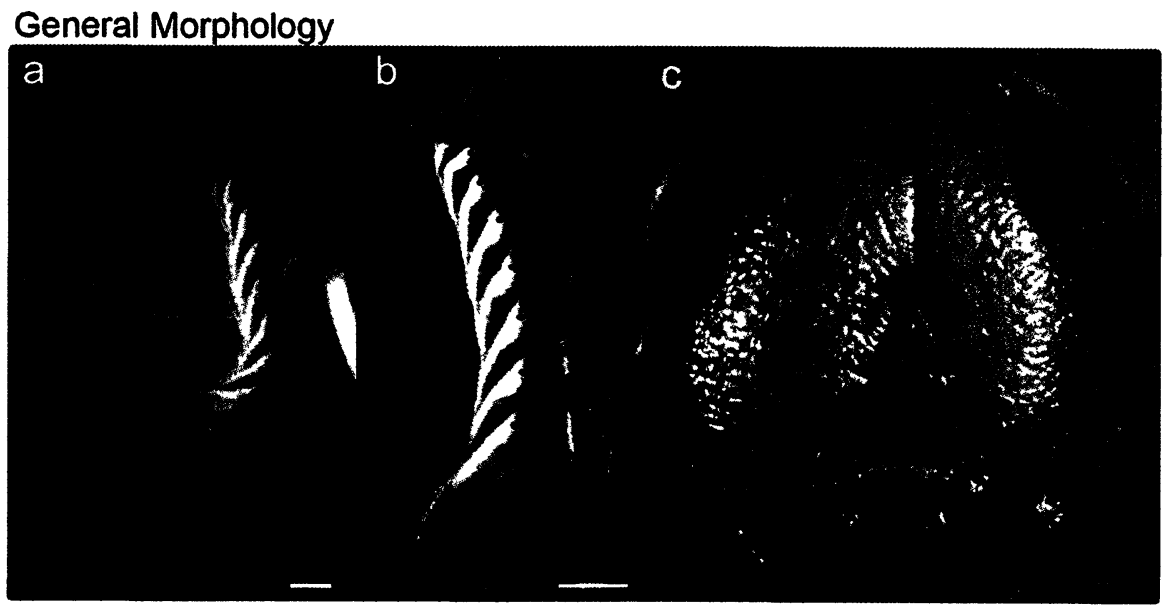

Mandibles

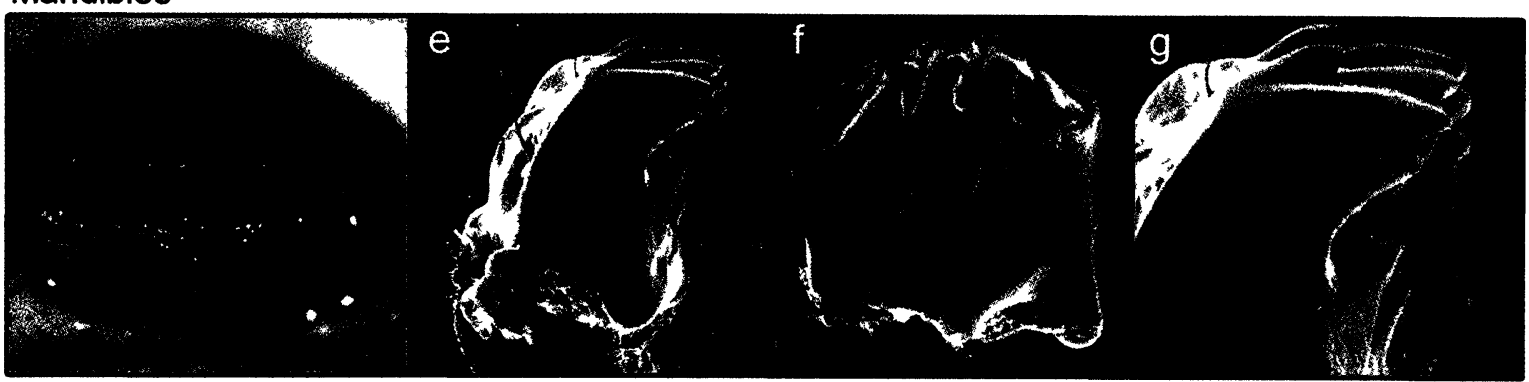

Defensive Behaviours

\begin{tabular}{|ll|}
\hline$\square$ Sound Production & $\square$ Biting \\
$\square$ Regurgitation & $\square$ Curlıng-in \\
$\square$ Chemical defense & $\square$ Curling up \\
$\square$ Thrashing & $\square$ Twitching \\
$\square$ Major thrashing & $\square$ Walk away \\
$\square$ Directed thrashing & $\square$ Dropping \\
\hline
\end{tabular}
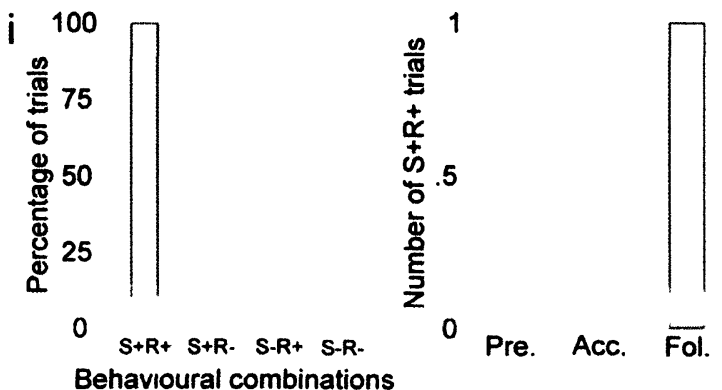

Figure 5.38 Manduca pellenia. General morphological features of a late instar caterpillar. (a) Lateral view of a late instar on its hostplant, scale bar $1 \mathrm{~cm}$. (b) Dorso-lateral view of a fifth instar caterpillar, scale bar $1 \mathrm{~cm}$. (c) Anterior view of the head capsule of a fifth instar, scale bar 0.5 $\mathrm{mm}$. Mandibles of a late instar caterpillar. (d) The mandibles of a late instar in closed position, scale bar $0.5 \mathrm{~mm}$; (e) right mandible, ventral-anterior view, scale bar $0.1 \mathrm{~mm}$; (f) left mandible, inner face, scale bar $0.1 \mathrm{~mm}$; (g) close up of anterior edge of right mandible, scale bar $0.1 \mathrm{~mm}$. (h) List of defensive behaviours observed upon attack and when handled. (i) Histograms showing the relationship between sound production (S) and regurgitation (R) during the first 5 pinches of an attack $(n=1)$. All images taken by Veronica Bura except (a) by Jayne Yack and (b) by Dan Janzen. 
a
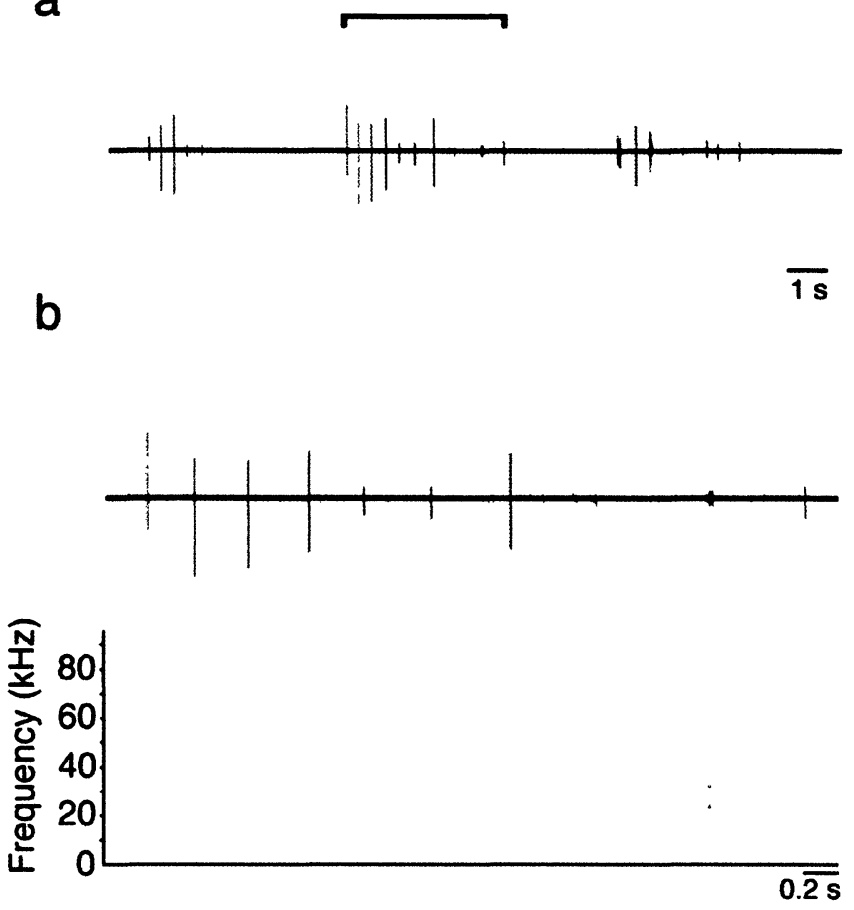

C

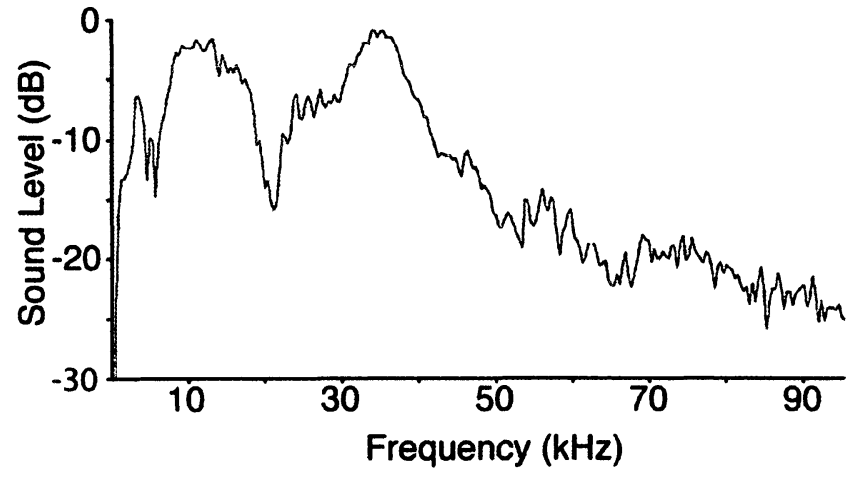

Figure 5.39 Sounds produced by late instar M. pellenia. (a) Oscillogram of clicks following attack. (b) Time expansion of bracket in (a) showing the individual components of each click. The accompanying spectrogram shows the frequency distribution of each click. (c) Power spectra taken from one individual. 
Manduca sexta (Linnaeus), the Tobacco Hornworm, Fig 5.40

Manduca sexta is a new world moth found in southern Canada and throughout much of the United States (Wagner 2005, Tuttle 2007). Tobacco Hornworms feed exclusively on members of the Solanaceae, including tomato (Solanum lycopersicum), potato (Solanum tuberosum) and datura (Datura sp.) (Wagner 2005, Tuttle 2007). Morphology: Caterpillars can be green, brown or black but can be distinguished by their white lateral stripes (Wagner 2005, Tuttle 2007). The larvae reared in the lab were green (Fig $5.40 \mathrm{a}, \mathrm{b}$ ), had a rounded head with an area of $22.39 \mathrm{~mm}^{2}$ (Fig $5.40 \mathrm{c}$ ) and ridged and serrated mandibles (Fig $5.40 \mathrm{e}-\mathrm{h}$ ). Caterpillars are capable of reaching $9 \mathrm{~cm}$ in length (Wagner 2005).

Behavioural Trials (Fig 5.40 i): Prior to experimentation the hornworms would rest along the mid-vein on the underside of the leaf. These caterpillars will feed during the day (Tuttle 2007). When attacked anteriorly the larvae would thrash away from the pinch, curl-in and produce sound. Subsequent pinches would cause the caterpillars to regurgitate (Fig $5.40 \mathrm{~d}$ ) and occasionally thrash from side to side.

Acoustics: Details on the sound characteristics, mechanism and associated behaviours have been reported in Chapter 2 of this thesis but are presented here for comparative purposes.

Sound characteristics: Trains of clicks were produced by $M$. sexta caterpillars when they were attacked (Fig $5.41 \mathrm{a}$ ). Trains were $3490 \pm 4920 \mathrm{~ms}$ in duration and contained 2-38 clicks ( $n=16$ trains from 16 animals). Individual clicks lasted $45.08 \pm 29.09 \mathrm{~ms}$ and contained 1-4 components (Fig 5.41 b). Sounds were broadband (Q3 $=4.71 \pm 0.19, \mathrm{Q} 10$ 
$=1.36 \pm 0.41, \mathrm{n}=15$ clicks from 3 animals) and had a dominant frequency of $28.05 \pm$ $9.85(\mathrm{n}=25$ clicks from 5 animals; Fig $5.41 \mathrm{c})$.

Mechanism: Video analysis and ablation experiments revealed that clicks are produced using the mandibles. The anterior and outer ridges of one mandible catch on the inner ridge of the other to produce individual clicks.

Behaviours associated with sound production and proposed function: Clicks were produced when the caterpillars were attacked on their head capsule and prolegs or when poked with a stiff piece of fishing line. Sounds were closely associated with regurgitation (Fig $5.40 \mathrm{j}$ ), and most often preceded its appearance. This coupling of defences suggests that clicks function to warn predators of an impending regurgitant defence. 


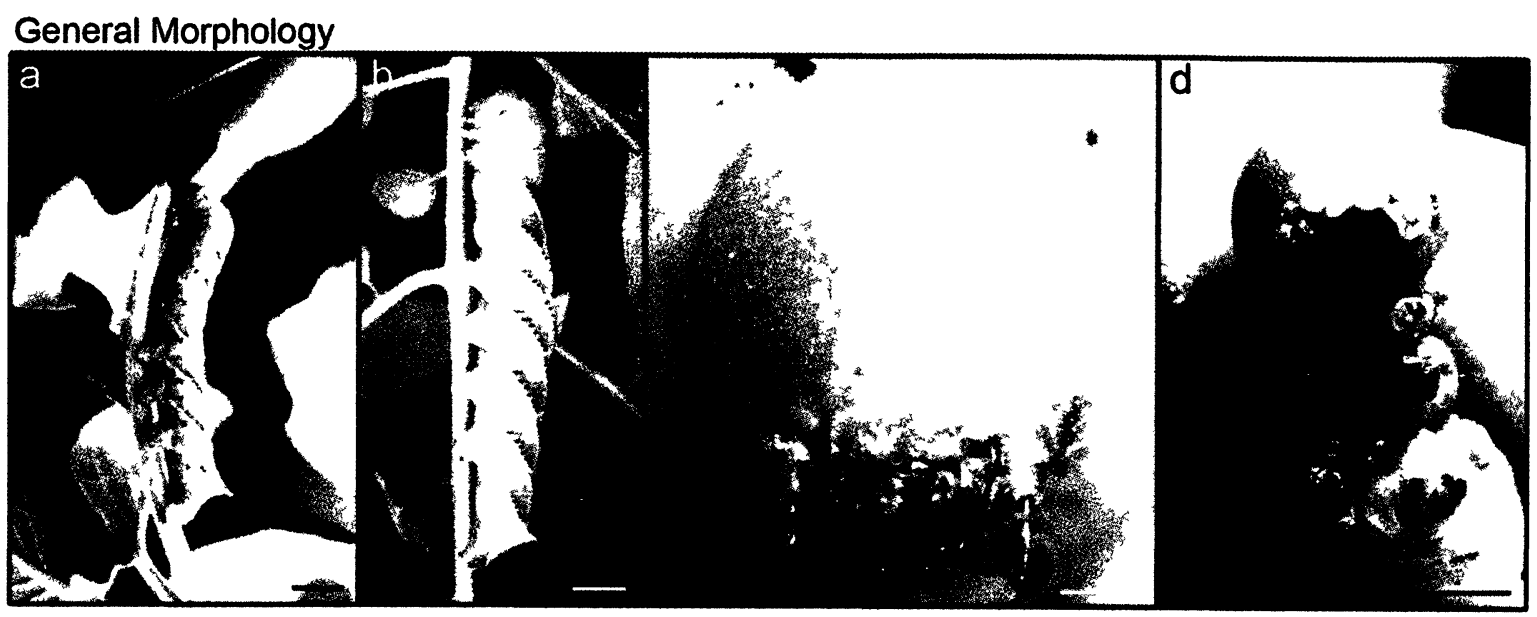

Mandibles

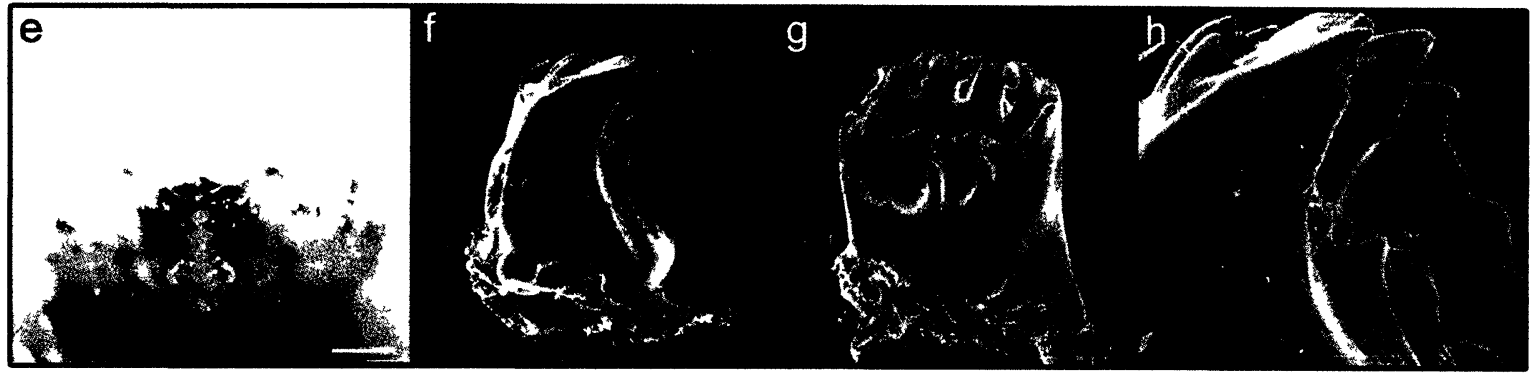

Defensive Behaviours

\begin{tabular}{|ll|}
\hline i $\square$ Sound Production & $\square$ Biting \\
$\square$ Regurgitation & $\square$ Curling-in \\
$\square$ Chemical defense & $\square$ Curling up \\
$\square^{\text {-h a ashing }}$ & $\square$ ditc ning \\
$\square$ Major thrashing & $\square$ Walk away \\
$\square$ Directed thrashing & $\square$ Dropping \\
\hline
\end{tabular}
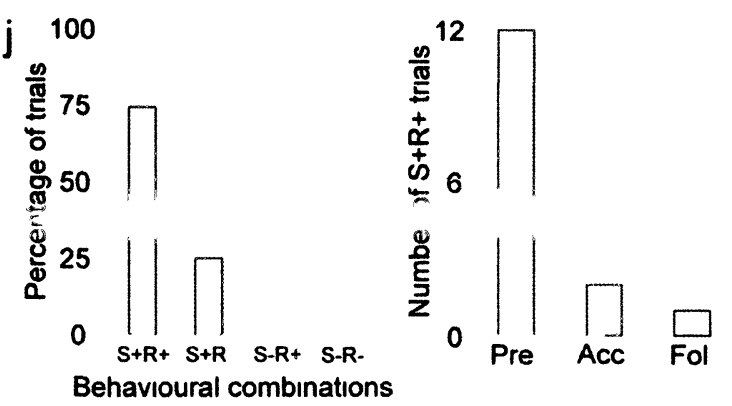

Figure 5.40 Manduca sexta, the Tobacco Hornworm. General morphological features of a late instar caterpillar. (a) Lateral view of a late instar larva on its hostplant, scale bar $0.5 \mathrm{~cm}$. (b) Lateral view of a fifth instar, scale bar $0.75 \mathrm{~cm}$. (c) Anterior view of the head capsule of a fifth instar, scale bar $1 \mathrm{~mm}$. (d) A late instar caterpillar regurgitating, scale bar $0.25 \mathrm{~cm}$. Mandibles of a late instar caterpillar. (e) The mandibles in closed position, scale bar $1 \mathrm{~mm}$; (f) right mandible, ventral-anterior view, scale bar $0.25 \mathrm{~mm}$; (g) left mandible, inner face, scale bar $0.25 \mathrm{~mm}$; (h) close up of anterior edge of right mandible, scale bar $0.1 \mathrm{~mm}$. (i) List of defensive behaviours observed upon attack and when handled. (j) Histograms showing the relationship between sound production (S) and regurgitation $(R)$ during the first 5 pinches of an attack $(n=20)$. All images taken by Veronica Bura, except (a) and (b) by Antoine Hnain and (d) and (e) by Jayne Yack. 
a

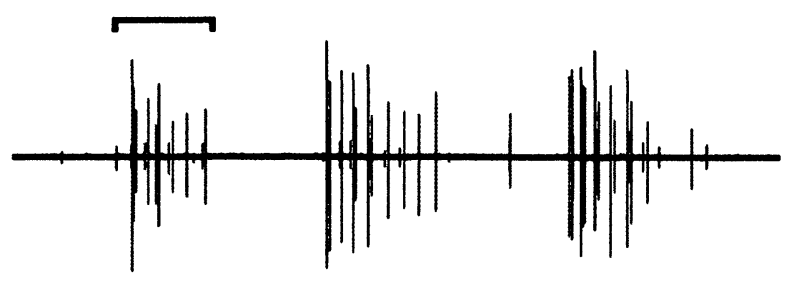

$\overline{1 s}$
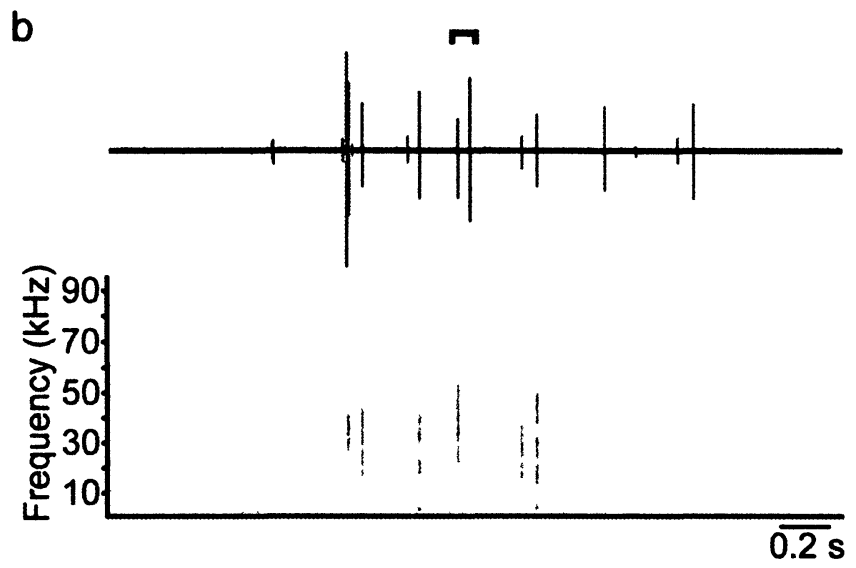

C

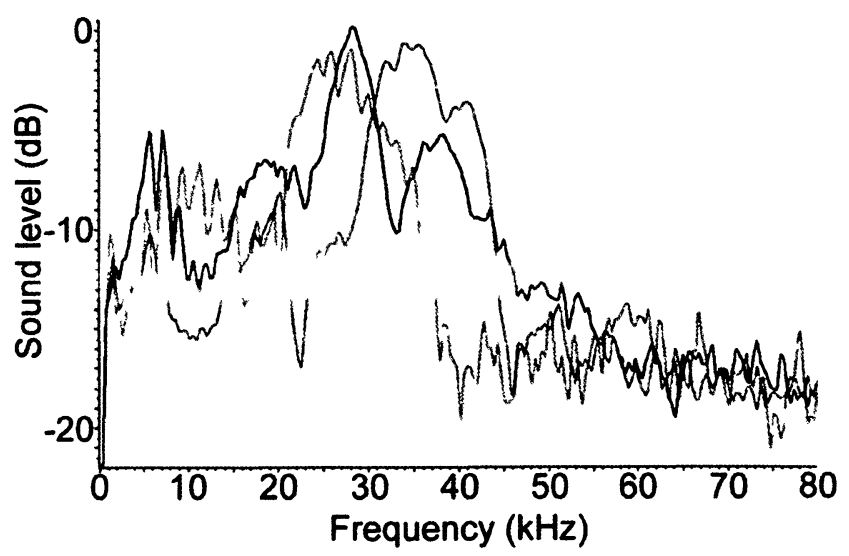

Figure 5.41 Sounds produced by late instar M. sexta caterpillars. (a) Oscillogram of clicks following attack. (b) Time expansion of bracket in (a) showing the multiple components of each click. The accompanying spectrogram shows the frequency distribution of each click. (c) Power spectra taken from three individuals. 


\section{Sphinx chersis (Hubner), the Great Ash sphinx, Fig 5.42}

The Great Ash sphinx is a new world moth found throughout much of North America (Wagner 2005, Tuttle 2007). Larvae of this species feed on members of

Oleaceae, Rosaceae and Salicaceae plant families, principally on ash (Fraxinus sp.) and other members of Oleaceae (Wagner 2005, Tuttle 2007).

Morphology: Caterpillars of the Great Ash sphinx can be green, bluish-green or slightly pink (Wagner 2005, Tuttle 2007). The single specimen I was able to acquire was a large green caterpillar (Fig $5.42 \mathrm{a}, \mathrm{b}) 5.8 \mathrm{~cm}$ in length, had an oval shaped head with an area of $33.91 \mathrm{~mm}^{2}$ (Fig $5.42 \mathrm{c}$ ) and ridged and serrated mandibles (Fig $5.42 \mathrm{~d}-\mathrm{g}$ ).

Behavioural Trials (Fig $5.42 \mathrm{~h}$ ): Late instar larvae rest along the mid-vein underneath the leaf, and actively feed during the day (Fig 5.42 a, Tuttle 2007). When attacked posteriorly the caterpillar curled-in and thrashed from side to side with its mandibles wide open, in theory preparing to bite the attacking forceps. The larva would thrash towards the forceps as they were making contact with its abdomen. Anterior attacks caused the same behaviours. When handled, this caterpillar would readily bite. No sound production was observed. Based on the observed defernses I suggest that the main defence used by this species is a combination of thrashing and biting. 


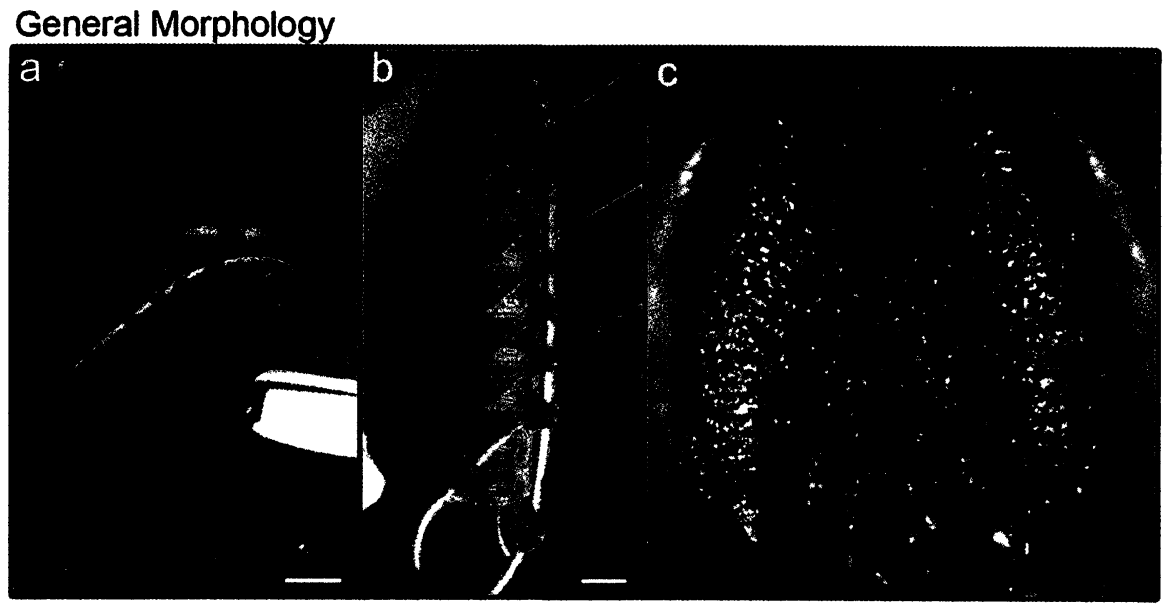

\section{Mandibles}

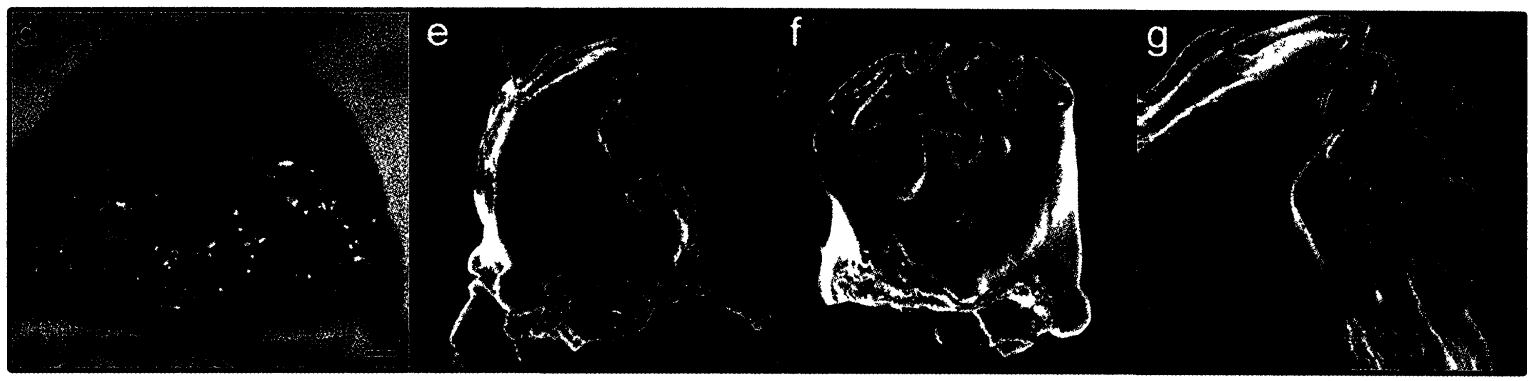

Defensive Behaviours

\begin{tabular}{|ll|}
\hline $\mathrm{h} \square$ Sound Production & $\square$ Biting \\
$\square$ Regurgitation & $\square$ Curling-in \\
$\square$ Chemical defense & $\square$ Curling up \\
$\square$ Thrashing & $\square$ Twitching \\
$\square$ Major thrashing & $\square$ Walk away \\
$\square$ Directed thrashing & $\square$ Droppıng \\
\hline
\end{tabular}

Figure 5.42 Sphinx chersis, the Great Ash sphinx. General morphological features of a late instar caterpillar. (a) Lateral view of a late instar on its hostplant, scale bar $1 \mathrm{~cm}$. (b) Lateral view of a fifth instar caterpillar, scale bar $0.5 \mathrm{~cm}$. (c) Anterior view of the head capsule of a fifth instar, scale bar $0.5 \mathrm{~mm}$. Mandibles of a late instar caterpillar. (d) The mandibles of a late instar in closed position, scale bar $0.5 \mathrm{~mm}$; (e) right mandible, ventral-anterior view, scale bar $0.25 \mathrm{~mm}$; (f) left mandible, inner face, scale bar $0.25 \mathrm{~mm}$; (g) close up of anterior edge of right mandible, scale bar $0.1 \mathrm{~mm}$. (h) List of defensive behaviours observed upon attack and when handled. All images taken by Veronica Bura. 
Sphinx drupiferarum (JE Smith), the Wild Cherry sphinx, Fig 5.43

The Wild Cherry sphinx is a new world moth found throughout much of North America (Wagner 2005, Tuttle 2007). Caterpillars of this species feed primarily on members of the Rosaceae family, especially cherries (Prunus sp.), but have also been noted on some Ulmaceae (Wagner 2005, Tuttle 2007).

Morphology: Late instar larvae of the Wild Cherry sphinx are green with a pink horn and pink and white lateral stripes (Fig $5.43 \mathrm{a}, \mathrm{b}$ ). They had an average length of $7.62 \mathrm{~cm}$ $(n=5)$, a rounded head with an area of $31.98 \mathrm{~mm}^{2}$ (Fig $5.43 \mathrm{c}$ ) and ridged and serrated mandibles (Fig $5.43 \mathrm{~d}-\mathrm{g}$ ).

Behavioural Trials (Fig 5.43 h): Sphinx drupiferarum caterpillars rest on the underside of the leaf along the mid-vein (Fig 5.43 a). When attacked posteriorly the caterpillars would thrash towards the site of attack, curl-in slightly and regurgitate. In half of these trials $(n=4)$ the caterpillar also thrashed from side to side. Anterior attacks caused the larvae to curl-in and thrash away from the pinch and continue thrashing from side to side. Regurgitation was also observed in these trials. This species would also bite when it was handled. No sound production was observed. 


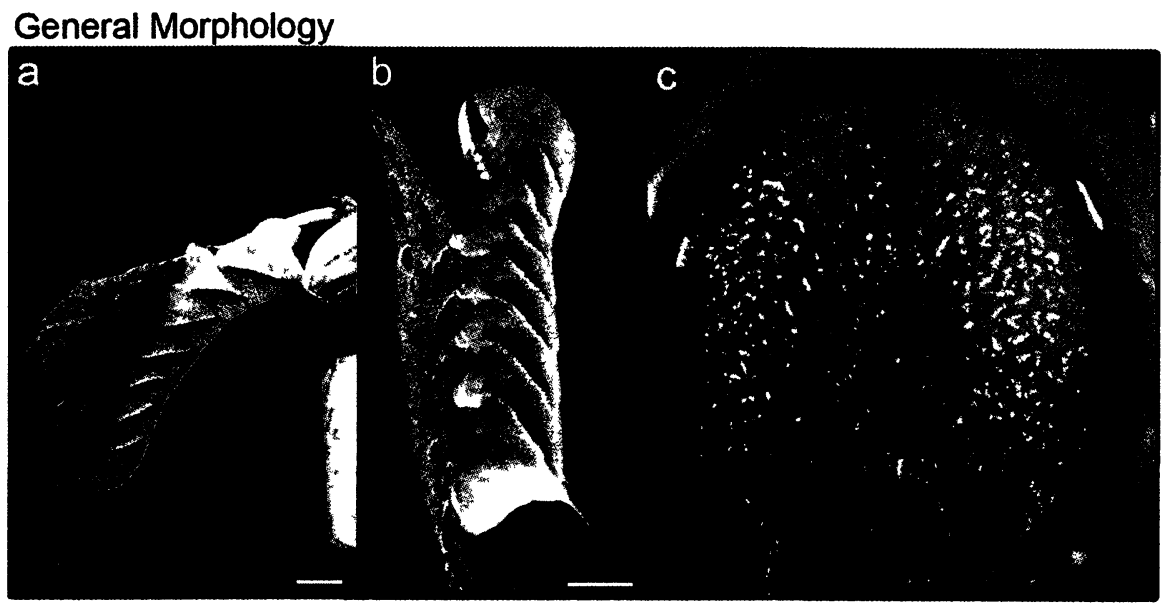

\section{Mandibles}

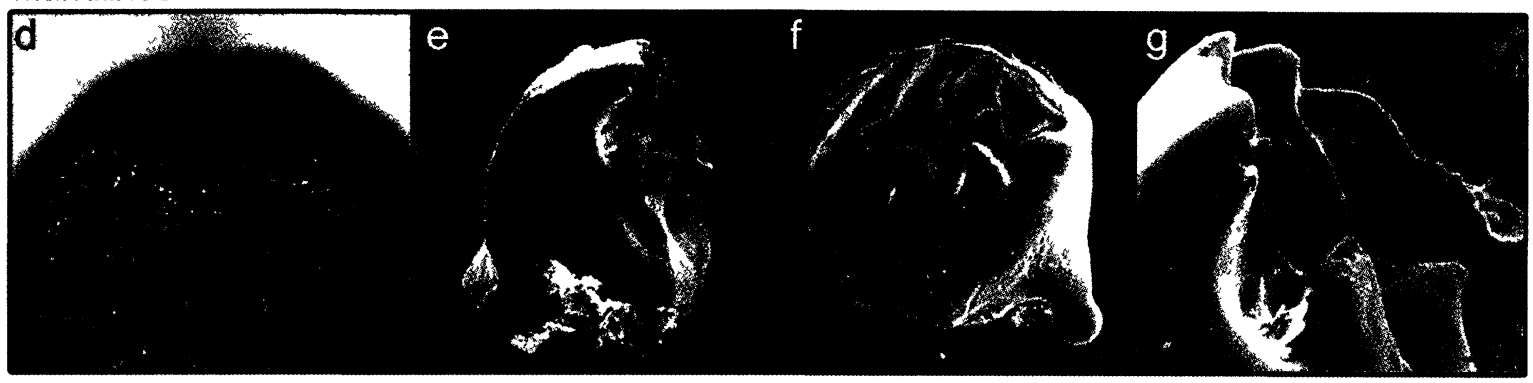

Defensive Behaviours

\begin{tabular}{|ll|}
\hline$\square$ Sound Production & Biting \\
$\square$ Regurgitation & $\square$ Curling-in \\
$\square$ Chemical defense & $\square$ Curling up \\
$\square$ Thrashing & $\square$ Twitching \\
$\square$ Major thrashing & $\square$ Walk away \\
$\square$ Directed thrashing & $\square$ Dropping \\
\hline
\end{tabular}

Figure 5.43 Sphinx drupiferarum, the Wild Cherry sphinx. General morphological features of a late instar caterpillar. (a) Lateral view of a late instar on its hostplant, scale bar $1 \mathrm{~cm}$. (b) Lateral view of a fifth instar caterpillar, scale bar $1 \mathrm{~cm}$. (c) Anterior view of the head capsule of a fifth instar, scale bar $0.5 \mathrm{~mm}$. Mandibles of a late instar caterpillar. (d) The mandibles of a late instar in closed position, scale bar $0.5 \mathrm{~mm}$; (e) right mandible, ventral-anterior view, scale bar $0.25 \mathrm{~mm}$; (f) left mandible, inner face, scale bar $0.25 \mathrm{~mm}$; (g) close up of anterior edge of right mandible, scale bar $0.1 \mathrm{~mm}$. (h) List of defensive behaviours observed upon attack and when handled. All images taken by Veronica Bura. 
Sphinx kalmiae (JE Smith), the Laurel sphinx, Fig 5.44

The Laurel sphinx is a new world moth found throughout eastern North America (Wagner 2005, Tuttle 2007). Caterpillars of this species feed on members of the Oleaceae, Betulaceae and Ericaceae families, commonly associated with ash species (Fraxinus sp.) (Wagner 2005, Tuttle 2007).

Morphology: Sphinx kalmiae caterpillars can be blue-green or yellow-green (Wagner 2005). The brood that I reared were more on the blue side with yellow and black lateral stripes (Fig 5.44 a,b). Fifth instar larvae were $6.96 \mathrm{~cm}$ in length $(\mathrm{n}=5)$, had oval shaped heads with an area of $22.29 \mathrm{~mm}^{2}$ (Fig $5.44 \mathrm{c}$ ) and ridged and serrated mandibles (Fig 5.44 $\mathrm{d}-\mathrm{g}$ ). These caterpillars are considered one of the most stunning sphingid larvae (Wagner 2005, Tuttle 2007).

Behavioural Trials: Laurel sphinx caterpillars rest on the underside on leaves along the mid-vein (Fig 5.44 b) and sever partially eaten leaves at their petioles (Wagner 2005). When attacked posteriorly caterpillars would curl-in, twitch and weakly thrash from side to side. Anterior attacks caused the larvae to curl-in, thrash away from the pinch and continue weakly thrashing from side to side. No sound production was observed. 


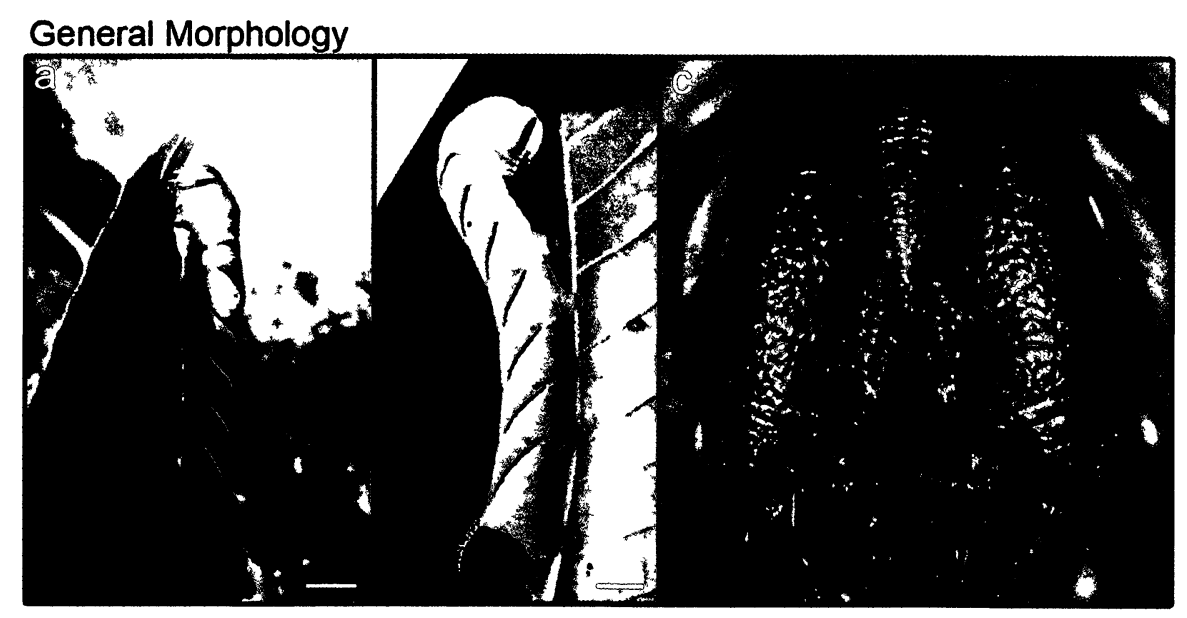

Mandibles

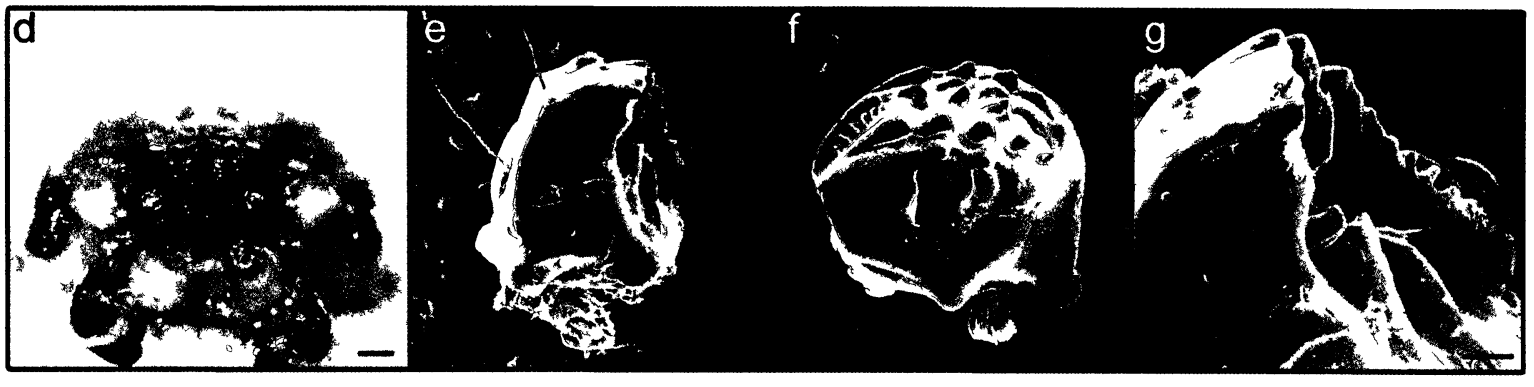

Defensive Behaviours

\begin{tabular}{|ll|}
\hline $\mathrm{h} \square$ Sound Production & $\square$ Bitıng \\
$\square$ Regurgitation & $\square$ Curling-in \\
$\square$ Chemical defense & $\square$ Curling up \\
$\square$ Thrashing & $\square$ Twitching \\
$\square$ Major thrashing & $\square$ Walk away \\
$\square$ Drrected thrashing & $\square$ Dropping \\
\hline
\end{tabular}

Figure 5.44 Sphinx kalmiae, the Laurel sphinx. General morphological features of a late instar caterpillar. (a) Lateral view of a late instar on its hostplant, scale bar $1 \mathrm{~cm}$. (b) Lateral view of a fifth instar caterpillar, scale bar $0.5 \mathrm{~cm}$. (c) Anterior view of the head capsule of a fifth instar, scale bar $0.5 \mathrm{~mm}$. Mandibles of a late instar caterpillar. (d) The mandibles of a late instar in closed position, scale bar $0.5 \mathrm{~mm}$; (e) right mandible, ventral-anterior view, scale bar $0.25 \mathrm{~mm}$; (f) left mandible, inner face, scale bar $0.25 \mathrm{~mm}$; (g) close up of anterior edge of right mandible, scale bar $0.1 \mathrm{~mm}$. (h) List of defensive behaviours observed upon attack and when handled. All images taken by Veronica Bura. 
Sphinx poecila Stephens, Fig 5.45

Sphinx poecila is a new world moth found throughout much of Canada and some northern United States (Tuttle 2007). Caterpillars feed on species from many plant families including Pinaceae, Myricaceae, Rosaceae and Ericaceae (Tuttle 2007). Morphology: Caterpillars of $S$. poecila can be green or a purplish wine colour with several white lateral stripes that are lined with purple in the green morph and black in the purple morph (Fig 5.45 a,b,d, Tuttle 2007). These caterpillars were very difficult to spot amongst the leaves of its hostplant. Fifth instar caterpillars were $5.76 \mathrm{~cm}$ in length $(\mathrm{n}=$ 5), had rounded heads with an area of $27.7 \mathrm{~mm}^{2}$ (Fig $5.45 \mathrm{c}$ ) and ridged and serrated mandibles (Fig 5.45 e-h).

Behavioural Trials (Fig 5.45 i): Sphinx poecila larvae feed during the day (Tuttle 2007) and rest on the underside of the leaves of their hostplant (Fig 5.45 a). When attacked posteriorly the caterpillars would thrash towards the site of attack and regurgitate. The larvae held their mandibles wide open as if to bite the attacking forceps. Anterior attacks caused the caterpillars to thrash away from the pinch and regurgitate, again holding its mandibles wide open. Some slight curling-in was observed on anterior attacks as well. This species would bite when handled. No sound production was observed. 
General Morphology

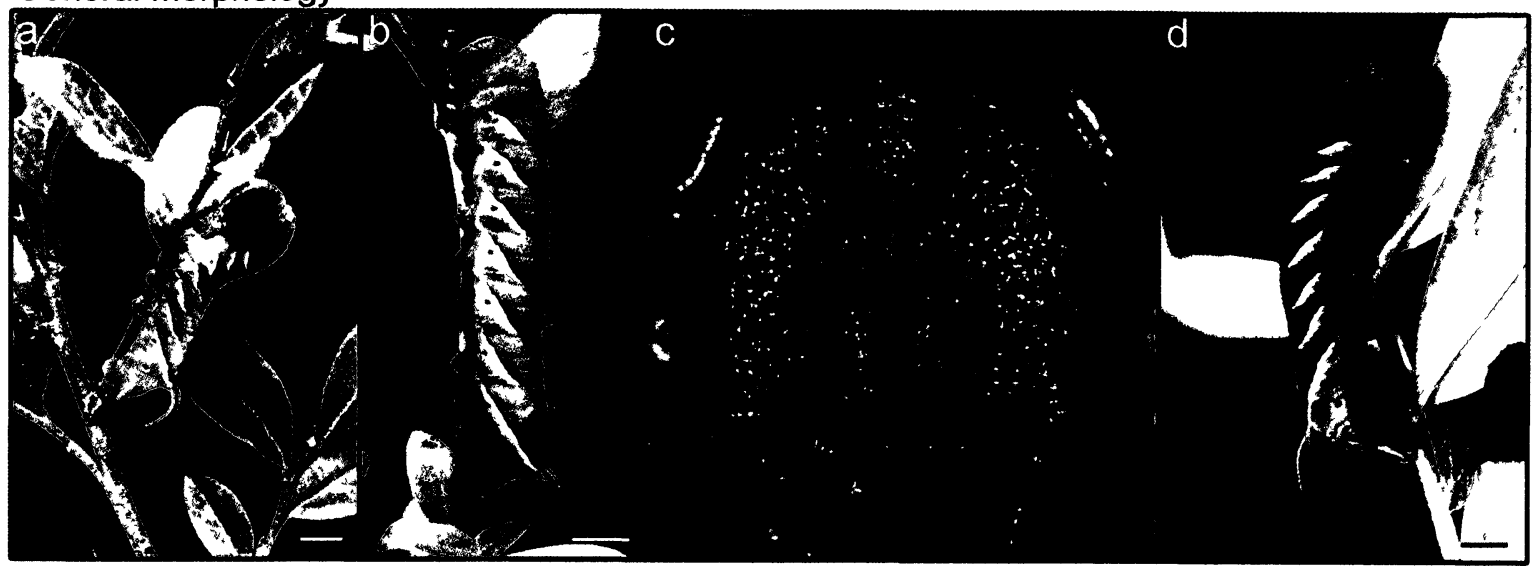

Mandibles

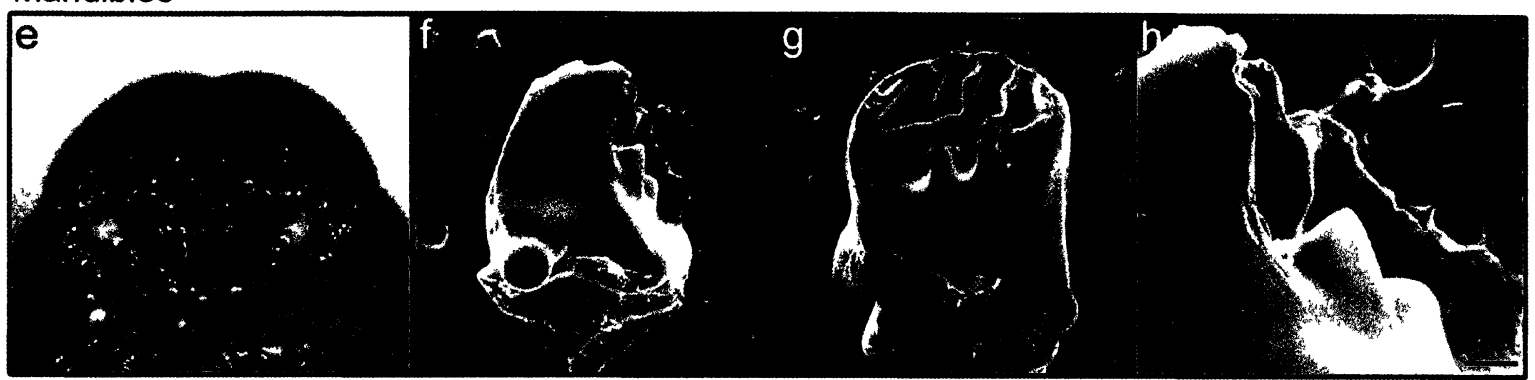

Defensive Behaviours

\begin{tabular}{|ll|}
\hline$\square$ Sound Production & $\square$ Biting \\
$\square$ Regurgitation & $\square$ Curling-in \\
$\square$ Chemical defense & $\square$ Curling up \\
$\square$ Thrashing & $\square$ Twitching \\
$\square$ Major thrashing & $\square$ Walk away \\
$\square$ Directed thrashing & $\square$ Dropping \\
\hline
\end{tabular}

Figure 5.45 Sphinx poecila. General morphological features of a late instar caterpillar. (a) Lateral view of a late instar on its hostplant, scale bar $0.5 \mathrm{~cm}$. (b) Lateral view of a fifth instar caterpillar, scale bar $0.75 \mathrm{~cm}$. (c) Anterior view of the head capsule of a fifth instar, scale bar 0.5 $\mathrm{mm}$. (d) A wine-coloured fifth instar morph, scale bar $0.5 \mathrm{~cm}$. Mandibles of a late instar caterpillar. (e) The mandibles of a late instar in closed position, scale bar $0.5 \mathrm{~mm}$; (f) right mandible, ventral-anterior view, scale bar $0.25 \mathrm{~mm}$; (g) left mandible, inner face, scale bar 0.25 $\mathrm{mm}$; (h) close up of anterior edge of right mandible, scale bar $0.1 \mathrm{~mm}$. (i) List of defensive behaviours observed upon attack and when handled. All images taken by Veronica Bura. 


\section{SUBFAMILY: MACROGLOSSINAE}

Caterpillars in some genera of this subfamily possess eyespots that create the appearance of a small snake (Kitching and Cadiou 2000). The eyespots can be exaggerated by enlarging body segments via head retraction or segment inflation (Kitching and Cadiou 2000).

\section{Darapsa choerilus (Cramer), the Azalea sphinx, Fig 5.46}

The Azalea sphinx is a new world moth found throughout eastern North American and central and western Canada (Wagner 2005, Tuttle 2007). This species feeds on azalea (Rhododendron sp.) and viburnum (Viburnum sp.), and has been reported on blueberry (Vaccinium sp.) and honeysuckle (Lonicera sp.) (Wagner 2005, Tuttle 2007).

Morphology: Azalea sphinx caterpillars have both brown and green colour morphs (Tuttle 2007). The specimens I reared were stout, green caterpillars with enlarged abdominal segments (Fig $5.46 \mathrm{a}, \mathrm{b})$. Fifth instar larvae had a mean length of $5 \mathrm{~cm}(\mathrm{n}=2)$, a small rounded head with an area of $10.94 \mathrm{~mm}^{2}$ (Fig $5.46 \mathrm{c}$ ) and ridged and serrated mandibles, the ridge on the inner face being blunt (Fig $5.46 \mathrm{e}-\mathrm{h}$ ).

Behavioural Trials (Fig 5.46 i): Darapsa choerilus larvae rest on the underside of the leaves of their hostplant and are difficult to find without a search image. Anterior attacks caused the caterpillars to curl-in to the extreme, retracting their head and thoracic segments into their abdomen (Fig $5.46 \mathrm{~d}$ ). While in this posture they would thrash away from the pinch and subsequently thrash slightly from side to side, twitch and regurgitate. When attacked posteriorly the same extreme curling-in occurred followed by twitching, regurgitation and slow directed thrashing towards the site of attack. No sound production was observed. 


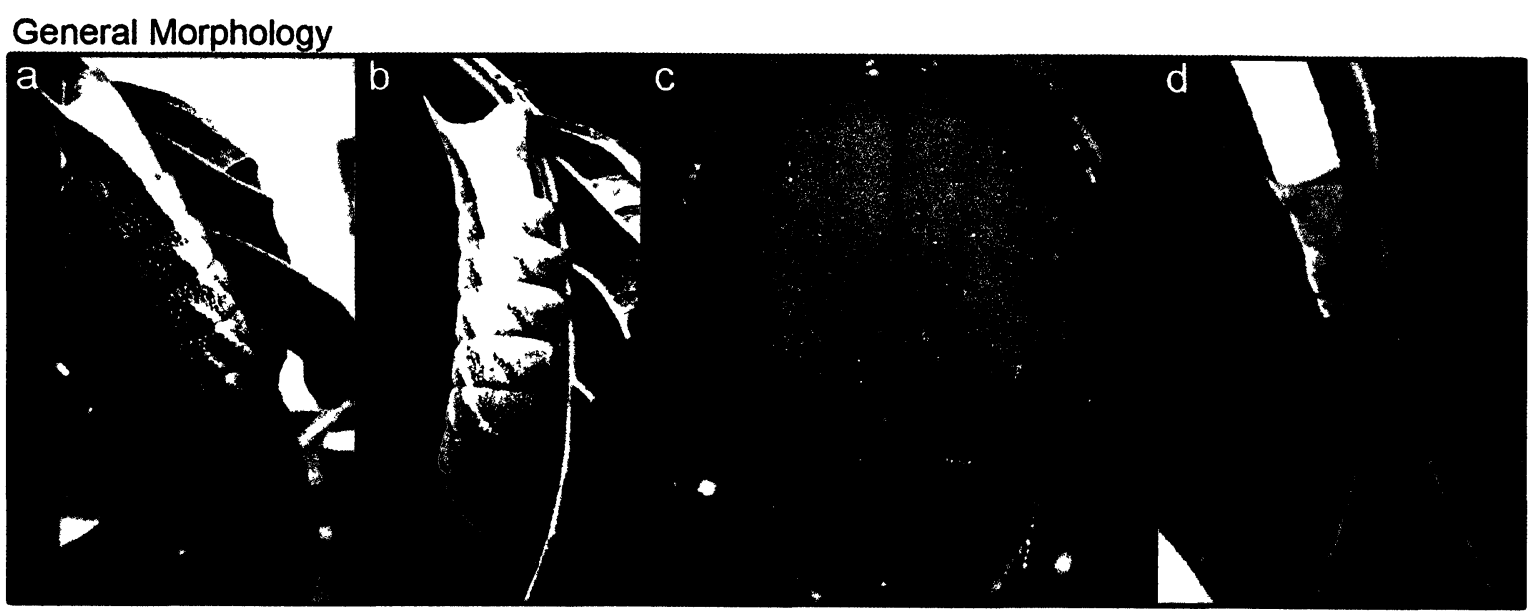

\section{Mandibles}

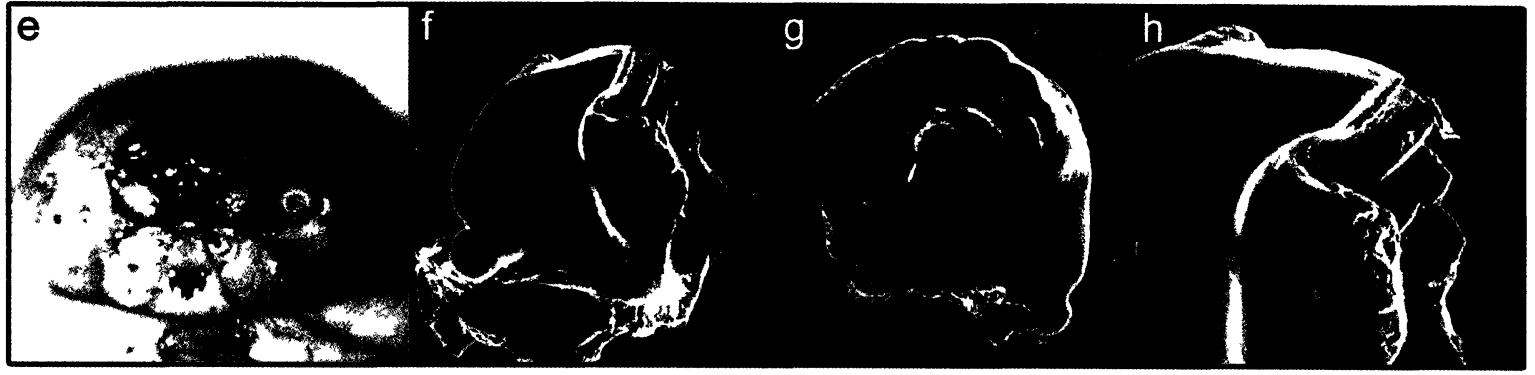

\section{Defensive Behaviours}

\begin{tabular}{|ll|}
\hline i $\square$ Sound Production & $\square$ Biting \\
$\square$ Regurgitation & $\square$ Curling-in \\
Chemical defense & $\square$ Curling up \\
$\square$ Major thrashing & $\square$ Twitching \\
$\square$ Directed thrashing & $\square$ Walk away \\
\hline
\end{tabular}

Figure 5.46 Darapsa choerilus, the Azalea sphinx. General morphological features of a late instar caterpillar. (a) Dorsal view of a fifth instar larva on its hostplant, scale bar $0.5 \mathrm{~cm}$. (b) Lateral view of a fifth instar caterpillar, scale bar $0.5 \mathrm{~cm}$. (c) Anterior view of the head capsule of a fifth instar, scale bar $0.5 \mathrm{~mm}$. (d) A fifth instar caterpillar with its head retracted, scale bar 0.5 $\mathrm{cm}$. Mandibles of a late instar caterpillar. (e) The mandibles of a late instar in closed position, scale bar $0.5 \mathrm{~mm}$; (f) right mandible, ventral-anterior view, scale bar $0.25 \mathrm{~mm}$; (g) left mandible, inner face, scale bar $0.25 \mathrm{~mm}$; (h) close up of anterior edge of right mandible, scale bar $0.1 \mathrm{~mm}$. (i) List of defensive behaviours observed upon attack and when handled. All images taken by Veronica Bura. 


\section{Darapsa myron (Cramer), the Hog sphinx, Fig 5.47}

The Hog sphinx is a new world moth found throughout eastern and central North America (Wagner 2005, Tuttle 2007). These caterpillars feed on members of the Vitaceae family, including grape (Vitis sp.), ampelopsis (Ampelopsis sp.) and Virginia creeper (Parthenocissus quinquefolia) (Wagner 2005, Tuttle 2007).

Morphology: Darapsa myron caterpillars can be yellow, blue-green, green or brown (Wagner 2005). The two specimens I was able to obtain were orange-brown (Fig $5.47 \mathrm{~b}$ ). The caterpillars had an average length of $4.8 \mathrm{~cm}(n=2)$, a small, rounded head with an area of $12.07 \mathrm{~mm}^{2}$ (Fig $5.47 \mathrm{c}$ ) and ridged and serrated mandibles (Fig $5.47 \mathrm{e}-\mathrm{h}$ ). This species also had enlarged abdominal segments.

Behavioural Trials (Fig 5.47 i): Hog sphinx larvae rest on the underside of the leaves of their hostplant along the mid-vein (Tuttle 2007). The specimens I was able to obtain were found as larva late in the season, and I only experimented with one. Before the attacks began, the head and thorax of the caterpillar was already retracted into its abdomen (i.e. was already showing some defensive behaviour). When attacked anteriorly the curled-in caterpillar would thrash from side to side from proleg 2 and regurgitate. Posterior attacks caused directed thrashing towards the site of attack followed by side to side thrashing and regurgitation. No sound production was observed. Please note, I am cautious about interpreting the results with this species as normal behaviour because it was found as a late instar larva. If the caterpillar was pre-pupal the behaviours may not be representative as behaviours change when this stage is reached (personal observation, e.g. Stamp 1986). 


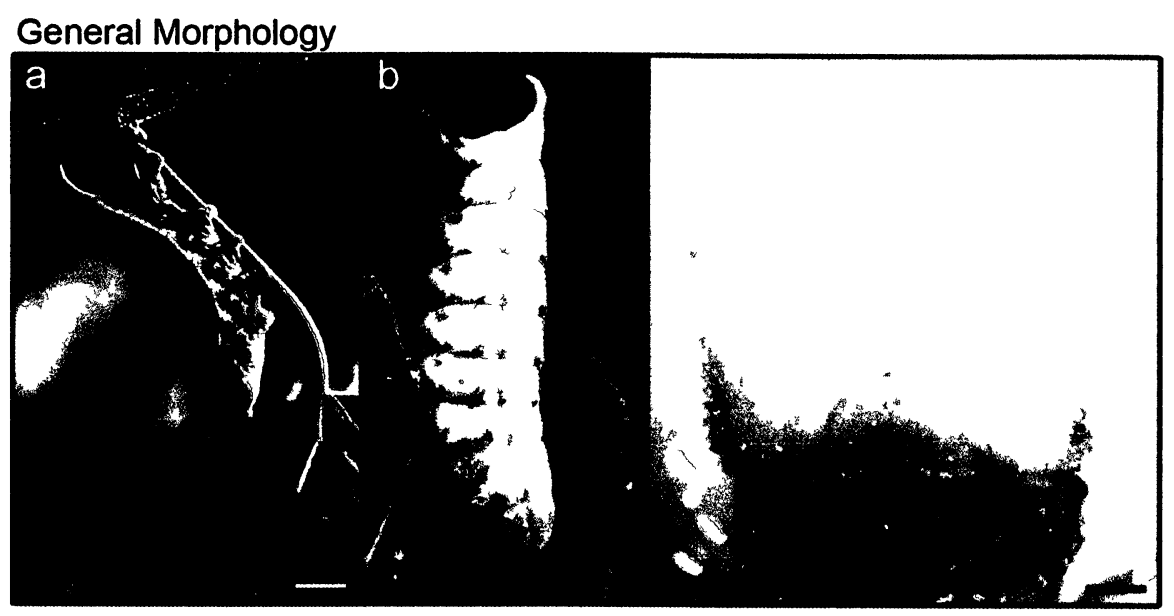

Mandibles

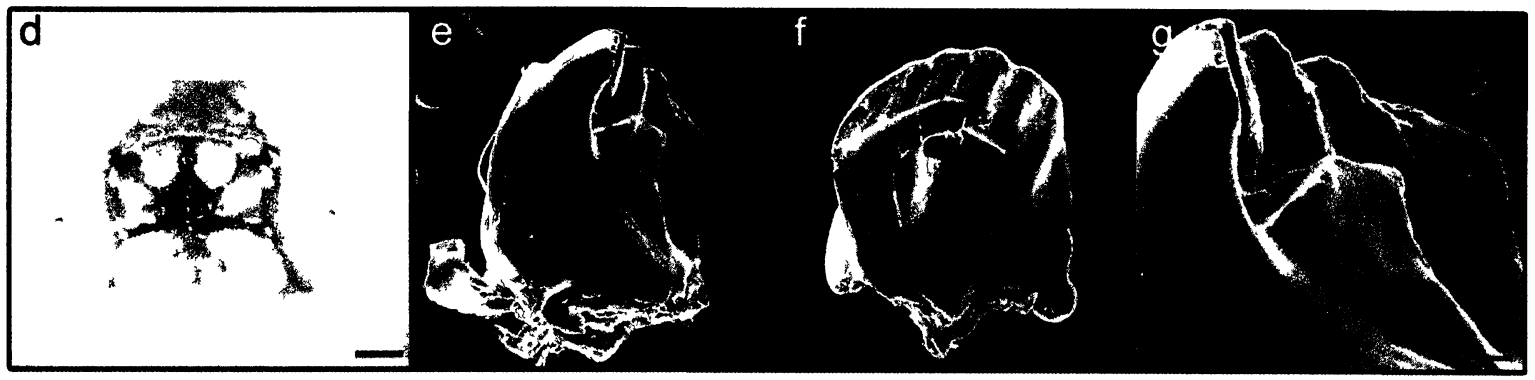

\section{Defensive Behaviours}

\begin{tabular}{|ll|}
\hline $\mathrm{h}$ Sound Production & $\square$ Biting \\
$\square$ Regurgitation & $\square$ Curling-in \\
$\square$ Chemical defense & $\square$ Curling up \\
$\square$ Thrashing & $\square$ Twitching \\
$\square$ Major thrashing & $\square$ Walk away \\
$\square$ Directed ihrasining & $\square$ Dropping \\
\hline
\end{tabular}

Figure 5.47 Darapsa myron, the Hog sphinx. General morphological features of a late instar caterpillar. (a) Lateral view of a late instar on its hostplant, scale bar $0.5 \mathrm{~cm}$. (b) Dorso-lateral view of a fifth instar caterpillar, scale bar $0.5 \mathrm{~cm}$. (c) Anterior view of the head capsule of a fifth instar, scale bar $0.5 \mathrm{~mm}$. Mandibles of a late instar caterpillar. (d) The mandibles of a late instar in closed position, scale bar $0.5 \mathrm{~mm}$; (e) right mandible, ventral-anterior view, scale bar $0.1 \mathrm{~mm}$; (f) left mandible, inner face, scale bar $0.1 \mathrm{~mm}$; (g) close up of anterior edge of right mandible, scale bar $0.1 \mathrm{~mm}$. (h) List of defensive behaviours observed upon attack and when handled. All images taken by Veronica Bura except (a) by Lance Risley (http://www.forestryimages.org/ browse/detail.cfm?imgnum=1791033). 
Deidamia inscriptum (Harris), the Lettered Sphinx, Fig 5.48

The lettered sphinx is a new world moth found throughout eastern North America (Wagner 2005, Tuttle 2007). Caterpillars feed on ampelopsis (Ampelopsis sp.), grape (Vitis sp.), woodbine (Parthenocissus vitacea) and Virginia creeper (Parthenocissus quinquefolia), all members of the Vitaceae family, as well as sourwood (Oxydendrum arboreum), a member of Ericaceae (Wagner 2005, Tuttle 2007).

Morphology: Deidamia inscriptum is a cryptic, green caterpillar with a sub-dorsal stripe on each side running the length of the body (Fig 5.48 a,b). I obtained a single specimen (n $=1$ ) that was about $4.5 \mathrm{~cm}$ long, had a small, rounded head (area $11.49 \mathrm{~mm}^{2}$, Fig $5.48 \mathrm{c}$ ) and ridged and serrated mandibles (Fig $5.48 \mathrm{e}-\mathrm{h}$ ).

Behavioural trials (Fig 5.48 i): The lettered sphinx larva was positioned along the midrib of the grape leaf prior to being attacked. It was resting with its body along the leaf and not in the typical 'sphinx' posture. When attacked anteriorly, the larva thrashed away from the pinch and retracted its head and part of its thorax into its abdomen, but not to the extent seen in other species (e.g. Darapsa choerilus, Darapsa myron, and Eumorpha satellitia). Subsequent pinches would cause the larva to thrash from side to side laterally, bringing its head next to its posterior end. Eventually the caterpillar dropped from the leaf, which could indicate that it was becoming prepupal or that it did not have a good purchase on the leaf. When replaced on the mid-vein of the leaf and attacked posteriorly the larva did not fall and demonstrated similar behaviours to the anterior attacks. It would thrash towards the site of attack and then laterally from side to side. No sound production was observed. 
Though I did not observe this in the single specimen I encountered, it has been reported that when disturbed the caterpillar will arch its head back over its abdomen and regurgitate (Fig 5.48 d) (Wagner 2005, Tuttle 2007), the specimen I had may have been approaching pupation which could explain the change in behaviour. 


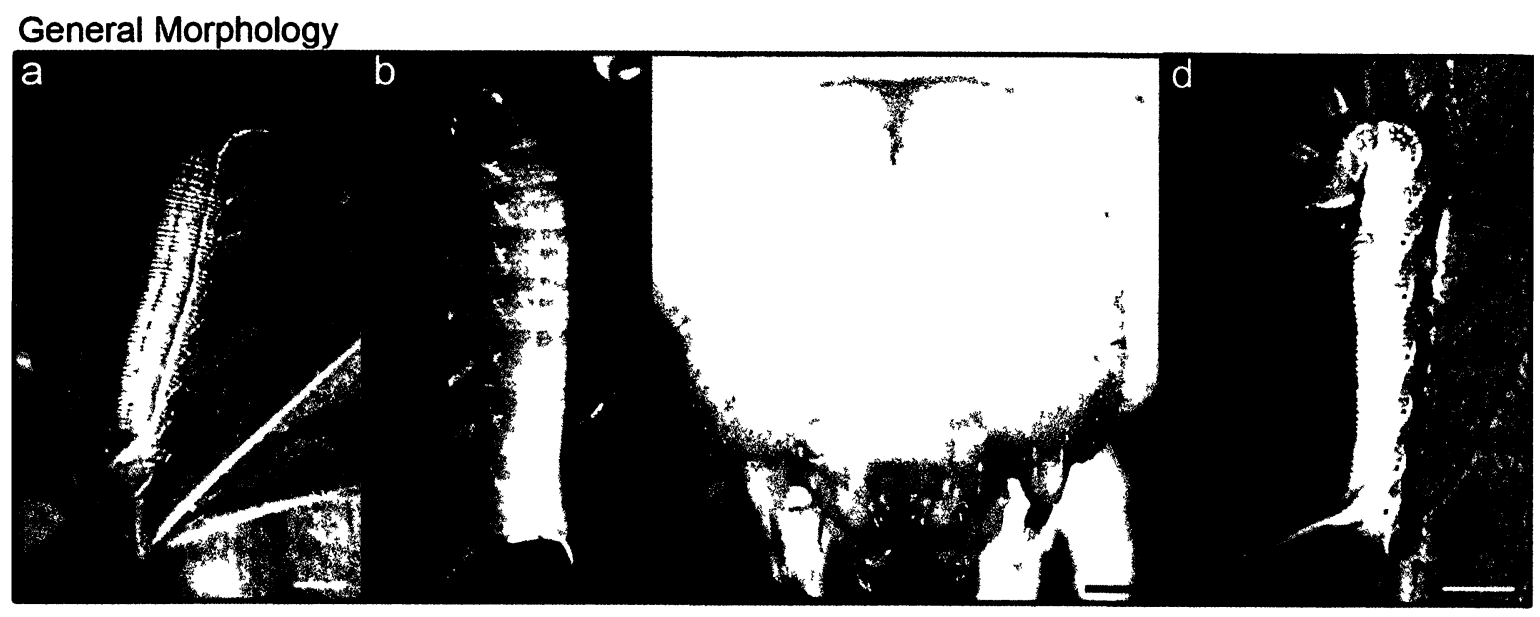

Mandibles

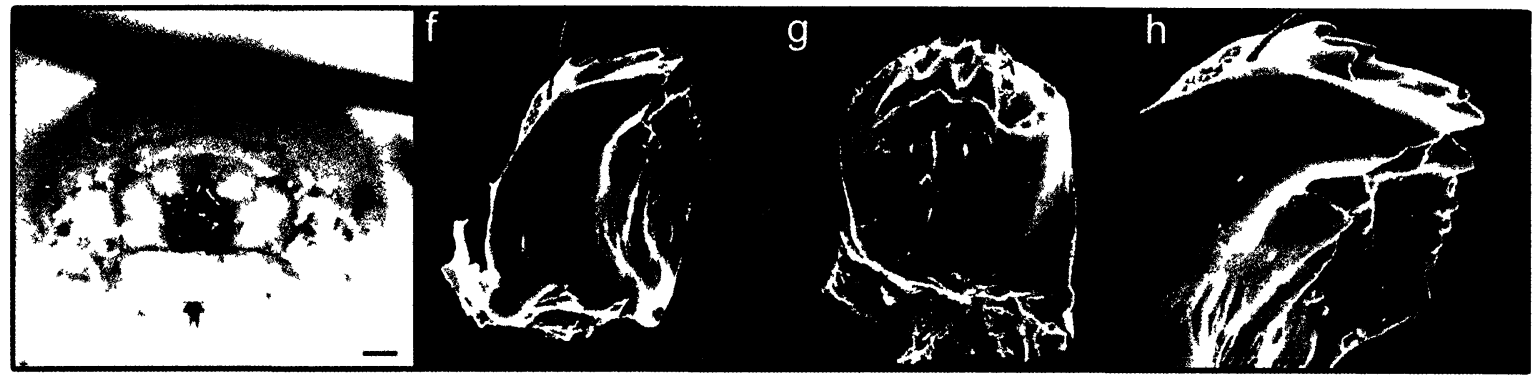

Defensive Behaviours

\begin{tabular}{|ll|}
\hline$\square$ Sound Production & $\square$ Biting \\
$\square$ Regurgitation & $\square$ Curling-in \\
$\square$ Chemical defense & $\square$ Curling up \\
$\square$ Thrashing & $\square$ Twitching \\
$\square$ Major thrashing & $\square$ Walk away \\
$\square$ Directed thrashing & $\square$ Dropping \\
\hline
\end{tabular}

Figure 5.48 Deidamia inscriptum, the Lettered sphinx. General morphological features of a late instar caterpillar. (a) Dorsal view of a late instar larva on its hostplant, scale bar $0.75 \mathrm{~cm}$. (b) Lateral view of a fifth instar caterpillar, scale bar $0.5 \mathrm{~cm}$. (c) Anterior view of the head capsule of a fifth instar, scale bar $0.5 \mathrm{~mm}$. (d) A late instar caterpillar in curled back defensive pose, scale bar $0.5 \mathrm{~cm}$. Mandibles of a late instar caterpillar. (e) The mandibles of a late instar in closed position, scale bar $0.5 \mathrm{~mm}$; (f) right mandible, ventral-anterior view, scale bar $0.25 \mathrm{~mm}$; (g) left mandible, inner face, scale bar $0.25 \mathrm{~mm}$; (h) close up of anterior edge of right mandible, scale bar $0.1 \mathrm{~mm}$. (i) List of defensive behaviours observed upon attack and when handled. All images taken by Veronica Bura, except (a) by Michael van Buskirk (http://www.silkmoths.bizland. com/dinscrip.htm) and (d) by M.J. Hatfield (http://bugguide.net/node/view/285026/bgimage). 
Eumorpha satellitia (Linnaeus), Fig 5.49

Eumorpha satellitia is a new world moth found in the southernmost regions of Texas and Florida, as well as throughout Costa Rica, northern Panama and southern Nicaragua (Tuttle 2007, Biodiversity occurrence data provided by: Instituto Nacional de Biodiversidad (Costa Rica) (Accessed through GBIF Data Portal, data.gbif.org, 2010-0408)). This species feeds on members of the grape family (Vitaceae) (Tuttle 2007, Janzen and Hallwachs 2009).

Morphology: Caterpillars of this species occur in two colour morphs, reddish brown and green (Fig $5.49 \mathrm{a}, \mathrm{b}$, Tuttle 2007). My green specimen was $8.5 \mathrm{~cm}$ in length, had a rounded head with an area of $30.91 \mathrm{~mm}^{2}$ (Fig $5.49 \mathrm{c}$ ), and had ridged and serrated mandibles, though the ridge on the inner face is not as prominent as in other species (Fig $5.49 \mathrm{e}-\mathrm{h})$.

Behavioural Trials (Fig 5.49 i): Late instar $E$. Satellitia larvae rest on the woody portion of their hostplant, revealing themselves only when they move to the foliage to eat (Tuttle 2007). The two morphs of this species that I tested reacted slightly differently to attack. The brown morph, when attacked posteriorly thrashed towards the site of attack and curled-in so that its head and thorax were retracted into its abdomen. On anterior attacks it thrashed away from the pinch and eventually started to walk away from the encounter. No sound production was observed. The green morph, when attacked posteriorly also thrashed towards the site of attack but subsequently would also thrash from side to side in a wide arc. This specimen also curled-in the same way but regurgitated and produced sound, a quiet clicking. When attacked anteriorly the same behaviours were observed. Acoustics: When attacked these caterpillars produced quiet clicks. 
Sound characteristics (Table 5.3): I was unable to obtain any high-sampled recordings of the sounds produced by these caterpillars, so details on the frequency characteristics of their signals are still unknown. Temporally, trains were $289.40 \pm 427.75 \mathrm{~ms}$ and contained $1-4$ clicks (Fig 5.50 a). Clicks lasted $40.89 \pm 36.65 \mathrm{~ms}$ and contained 1-4 components (Fig $5.50 \mathrm{~b}$ ).

Mechanism: As with other clicking species, I suspect that clicks are produced using the mandibles. 


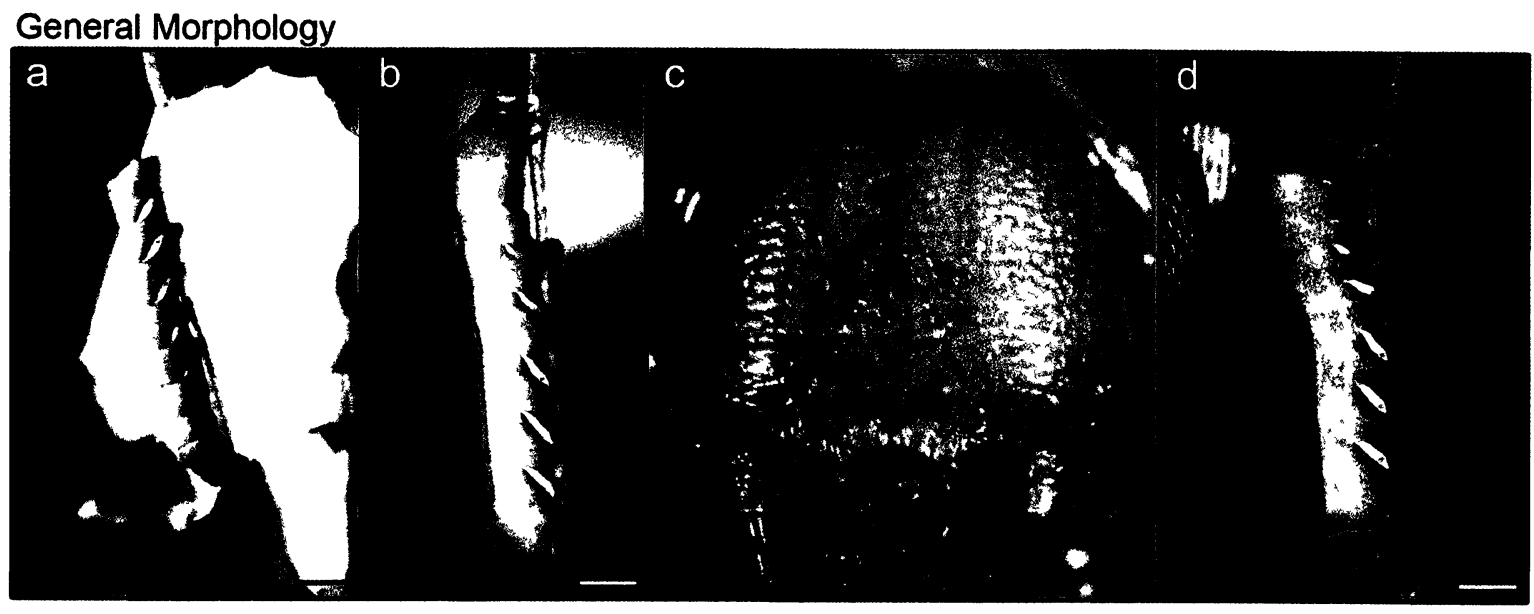

Mandibles

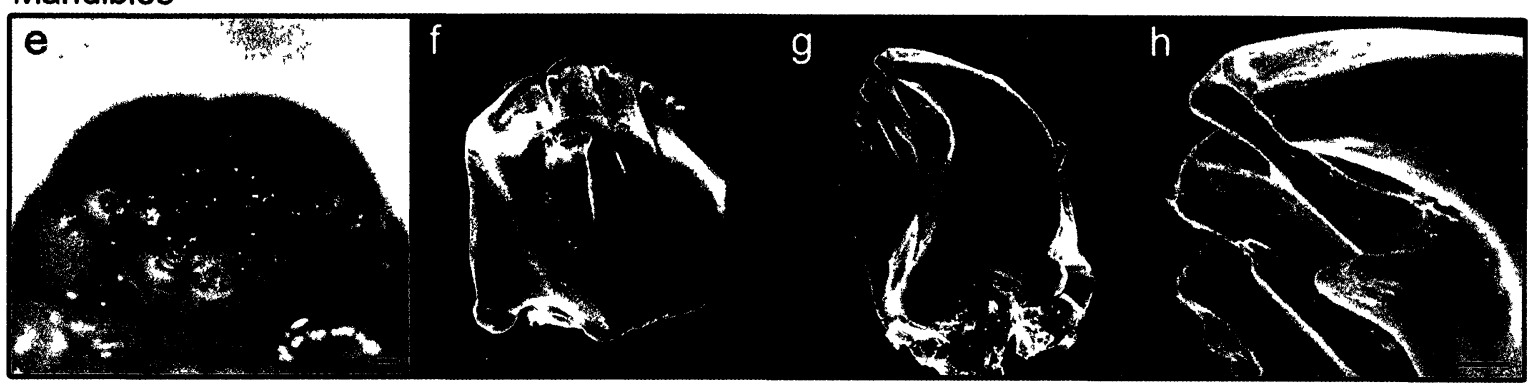

Defensive Behaviours

\begin{tabular}{|ll|}
\hline i $\square$ Sound Production & $\square$ Biting \\
$\square$ Regurgitation & $\square$ Curling-in \\
$\square$ Chemical defense & $\square$ Curling up \\
$\square$ Thrashing & $\square$ Twitching \\
$\square$ Major thrashing & $\square$ Walk away \\
$\square$ Directed thrashing & $\square$ Dropping \\
\hline
\end{tabular}

Figure 5.49 Eumorpha satellitia. General morphological features of a late instar caterpillar. (a) Lateral view of a late instar larva on its hostplant, anterior is down, scale bar $1 \mathrm{~cm}$. (b) Lateral view of a fifth instar caterpillar, scale bar $1 \mathrm{~cm}$. (c) Anterior view of the head capsule of a fifth instar, scale bar $0.5 \mathrm{~mm}$. (d) A late instar caterpillar with head retracted, scale bar $1 \mathrm{~cm}$. Mandibles of a late instar caterpillar. (e) The mandibles of a late instar in closed position, scale bar $0.5 \mathrm{~mm}$; (f) right mandible, inner face, scale bar $0.25 \mathrm{~mm}$; (g) left mandible, ventral-anterior view, scale bar $0.25 \mathrm{~mm}$; (h) close up of anterior edge of left mandible, scale bar $0.1 \mathrm{~mm}$. (i) List of defensive behaviours observed upon attack and when handled. All images taken by Veronica Bura, except (a) by Jayne Yack and (b) by Alan Fleming. 


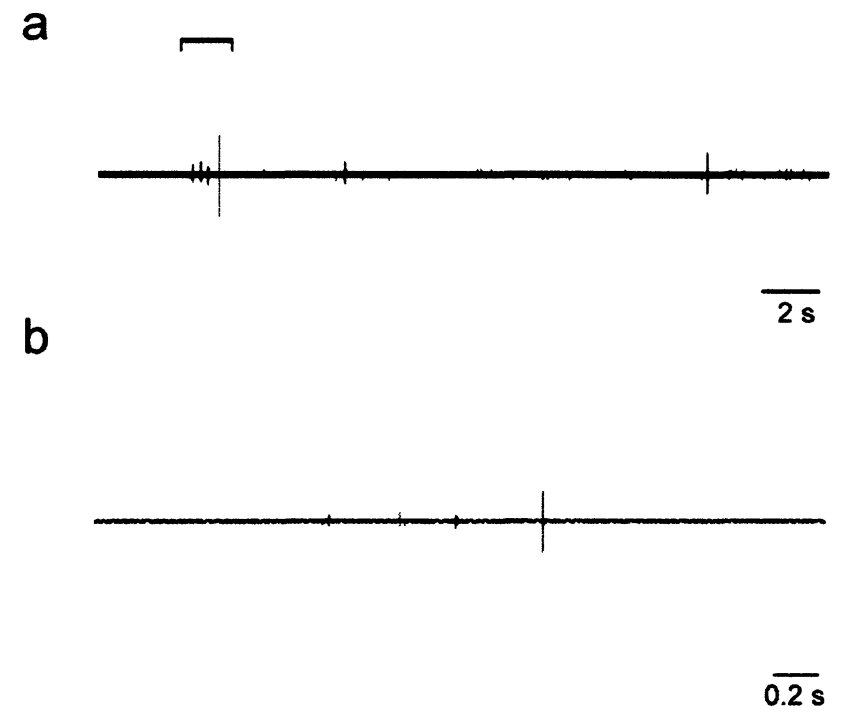

Figure 5.50 Sounds produced by late instar $\boldsymbol{E}$. satellitia caterpillars. (a) Oscillogram of clicks following attack. (b) Time expansion of bracket in (a). 
Nyceryx magna (Felder), Fig 5.51

Nyceryx magna is a new world moth found in Costa Rica, Brazil and Ecuador (Oehkle 2009, Savela 2009). Caterpillars feed on Pentagonia donnell-smithii, a member of the Rubiaceae family (Janzen and Hallwachs 2009).

Morphology: The fifth instar caterpillar that I tested was green with a black stripe running dorsally, surrounded by yellow on either side (Fig $5.51 \mathrm{a}, \mathrm{b})$. Its caudal horn was a pinkish-red and its rounded head was bright blue and $27.59 \mathrm{~mm}^{2}$ in area (Fig $5.51 \mathrm{c}$ ). The larva was $6.8 \mathrm{~cm}$ in length and had ridged and serrated mandibles (Fig $5.51 \mathrm{~d}-\mathrm{g}$ ). Behavioural Trials (Fig $5.51 \mathrm{~h}$ ): The caterpillar rested along the mid-vein on the underside of the leaf (Fig $5.51 \mathrm{a}$ ). When attacked anteriorly the caterpillar thrashed away from the pinch and attempted to leave the area, but stopped when it was attacked again. The larva then began to produce sound and thrash from side to side. Posterior attacks also elicited sound production and the caterpillar would thrash towards the site of attack and then back and forth at a rate of approximately 3.16 flicks per second from either the $3^{\text {rd }}$ or $4^{\text {th }}$ proleg. The caterpillar regurgitated when handled.

Acoustics: The sounds produced by this caterpillar resembled a rasp at times and a squeak at other times.

Sound characteristics (Table 5.3): The acoustic signals produced by $N$. magna consist of trains of sounds that were $3860 \pm 1392 \mathrm{~ms}$ in duration, containing 8-15 rasps (Fig 5.52 $\mathrm{a}, \mathrm{b}, \mathrm{n}=4$ trains from 1 animal). Rasps were $50.39 \pm 11.16 \mathrm{~ms}$ in duration and contained 6-21 components $(n=46$ sound pulses). Sounds were broadband (mean $Q 3=6.20 \pm 7.09$, $\mathrm{Q} 10=1.33 \pm 1.31, \mathrm{n}=5$ sounds from 1 animal) and had a mean dominant frequency of $24.83 \pm 12.51 \mathrm{kHz}$ (Fig $5.52 \mathrm{c})$. 
Mechanism: Video analysis revealed that sounds are produced when the mandibles are open, much like the mechanism used by the Abbott's sphinx (see below). Unlike the Abbott's sphinx, however, there did not appear to be any click portion of the signal. Behaviours associated with sound production and proposed function: Sounds were produced as the caterpillar thrashed from side to side. It was difficult to get the larva to signal when it was unable to move. The lack of regurgitation associated with signalling suggests the sounds may function as a startle signal (Fig $5.51 \mathrm{i}$ ). However, the speed at which this caterpillar was capable of thrashing could make it more difficult and time consuming to obtain, so these sound may also function as a general warning of unprofitability, indicating the time it would take to capture the caterpillar would not be worth it. 


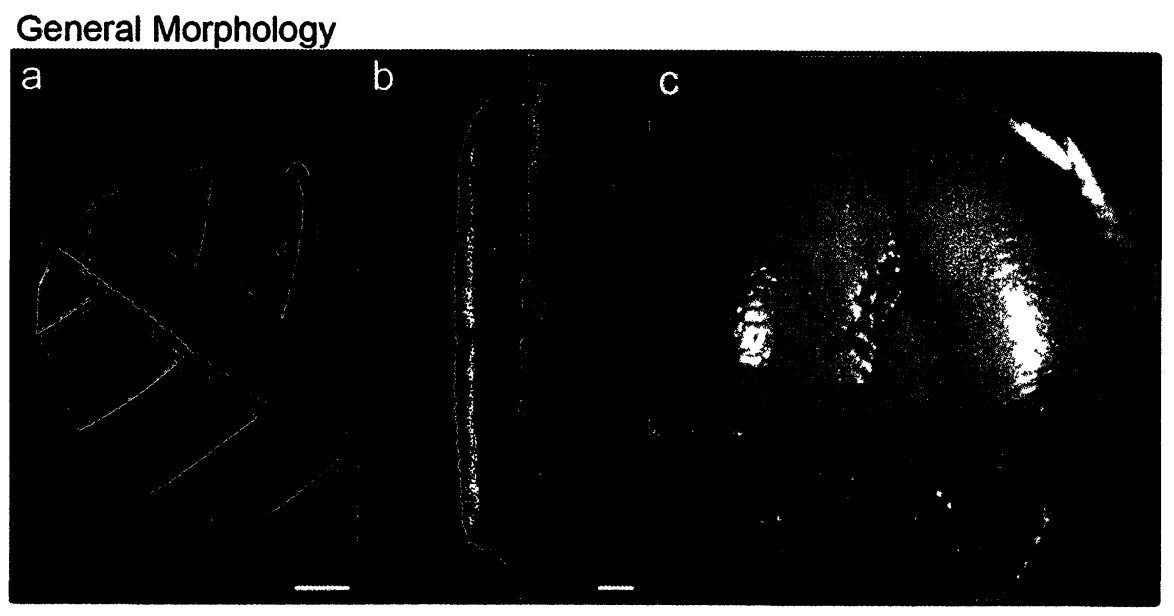

Mandibles

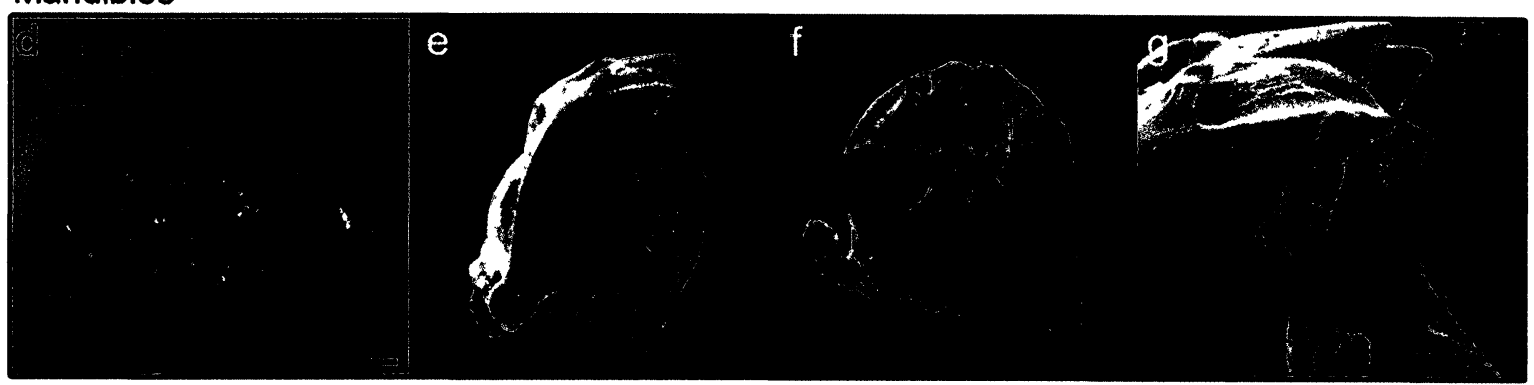

\section{Defensive Behaviours}

\begin{tabular}{|ll|}
\hline h日 Sound Production & $\square$ Biting \\
$\square$ Regurgitation & $\square$ Curling-in \\
$\square$ Chemical defense & $\square$ Curling up \\
$\square$ Thrashing & $\square$ Twitching \\
$\square$ Major thrashing & $\square$ Walk away \\
$\square$ Orected thrashing & $\square$ Dropping \\
\hline
\end{tabular}

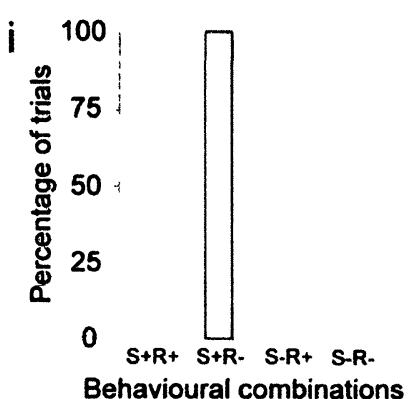

Figure 5.51 Nyceryx magna. General morphological features of a late instar caterpillar. (a) Dorsal view of a late instar on its hostplant, scale bar $1 \mathrm{~cm}$. (b) Lateral view of a fifth instar caterpillar, scale bar $0.5 \mathrm{~cm}$. (c) Anterior view of the head capsule of a fifth instar, scale bar 0.5 $\mathrm{mm}$. Mandibles of a late instar caterpillar. (d) The mandibles of a late instar in closed position, scale bar $0.5 \mathrm{~mm}$; (e) right mandible, ventral-anterior view, scale bar $0.25 \mathrm{~mm}$; (f) left mandible, inner face, scale bar $0.25 \mathrm{~mm}$; (g) close up of anterior edge of right mandible, scale bar $0.1 \mathrm{~mm}$. (h) List of defensive behaviours observed upon attack and when handled. (i) Histogram showing the relationship between sound production (S) and regurgitation (R) during the first 5 pinches of an attack $(n=1)$. All images taken by Veronica Bura except (a) and (b) by Alan Fleming. 
a

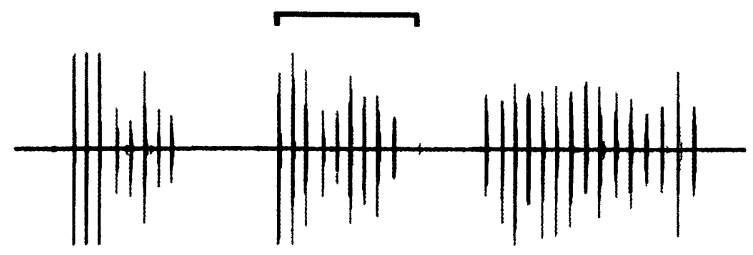

b
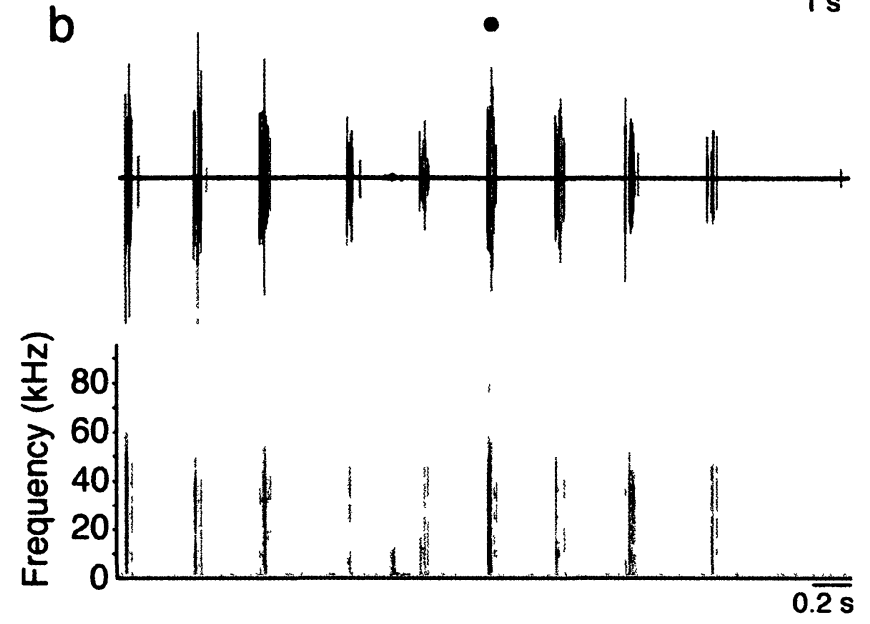

C

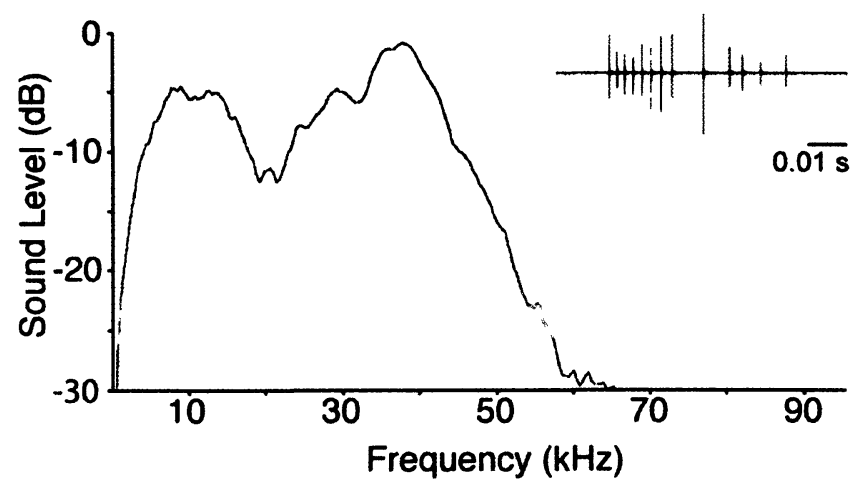

Figure 5.52 Sounds produced by late instar $N$. magna caterpillars. (a) Oscillogram of rasps following attack. (b) Time expansion of bracket in (a). The accompanying spectrogram shows the frequency distribution of each rasp. (c) Power spectrum taken from one individual. Further expanded time scale of rasp (dot in b) is inset showing the multiple components. 
Pachygonidia drucei (Rothschild \& Jordan), Fig 5.53

Pachygonidia drucei is a new world moth found from southern Mexico, through Central America south to Ecuador (Oehkle 2009, Savela 2009). Caterpillars feed on Doliocarpus dentatus, D. multiflorus and Tetracera hydrophila, all members of the Dilleniaceae family (Janzen and Hallwachs 2009).

Morphology: Pachygonidia drucei caterpillars are green and have a brown vein-like pattern on their dorsal surface (Fig $5.53 \mathrm{a}, \mathrm{b}$ ). They lose their horn in their final instar and can reach lengths of $6.5 \mathrm{~cm}$ (Janzen and Hallwachs 2009).

Behavioural Trials (Fig 5.53 c): Prior to the trial, the $P$. drucei caterpillar was resting along the mid-vein on the underside of the leaf. When attacked posteriorly the larva would respond with rasping sounds, slight directed thrashing, twitching and slow winding thrashing towards the posterior end. Winding thrashes were preceded by twitching. Anterior attacks caused the caterpillar to regurgitate a lot right away, thrash away from the pinch and then in a wide arc from side to side. Less sound production was observed on this attack.

Acoustics: Sounds produced by the $P$. drucei caterpillar seemed quieter to the unaided human ear than those of S. abbottii and N. magna, which produced similar, rasp-type sounds.

Sound characteristics (Table 5.3): The acoustic signals produced by $P$. drucei consist of trains of sounds that were $176.75 \pm 23.80 \mathrm{~ms}$ in duration, containing 1-2 rasps (Fig 5.54 $\mathrm{a}, \mathrm{b}, \mathrm{n}=5$ trains from 1 animal). Rasps were $102.44 \pm 85.51 \mathrm{~ms}$ in duration and contained $2-43$ components ( $n=8$ sound pulses). Sounds were broadband (mean Q3 $=2.85 \pm 2.30$, 
$\mathrm{Q} 10=0.69 \pm 0.24, \mathrm{n}=5$ sounds from 1 animal) and had a mean dominant frequency of $42.90 \pm 10.68 \mathrm{kHz}$ (Fig $5.54 \mathrm{c})$.

Mechanism: Video analysis revealed that sounds are produced when the mandibles are open, much like the mechanism used by the Abbott's sphinx (see below). Again, there did not appear to be any 'click' portion in the signals of this caterpillar. Behaviours associated with sound production and proposed function: Sound production occurred during the winding thrashes of the caterpillar. During the first five pinches of the attack, no regurgitation was observed (Fig $5.53 \mathrm{~d}$ ). A lot of regurgitation did occur later in the trial, however, after the caterpillar had been attacked for a long period of time. Further investigations are required to determine the relationship between behaviours. 
General Morphology
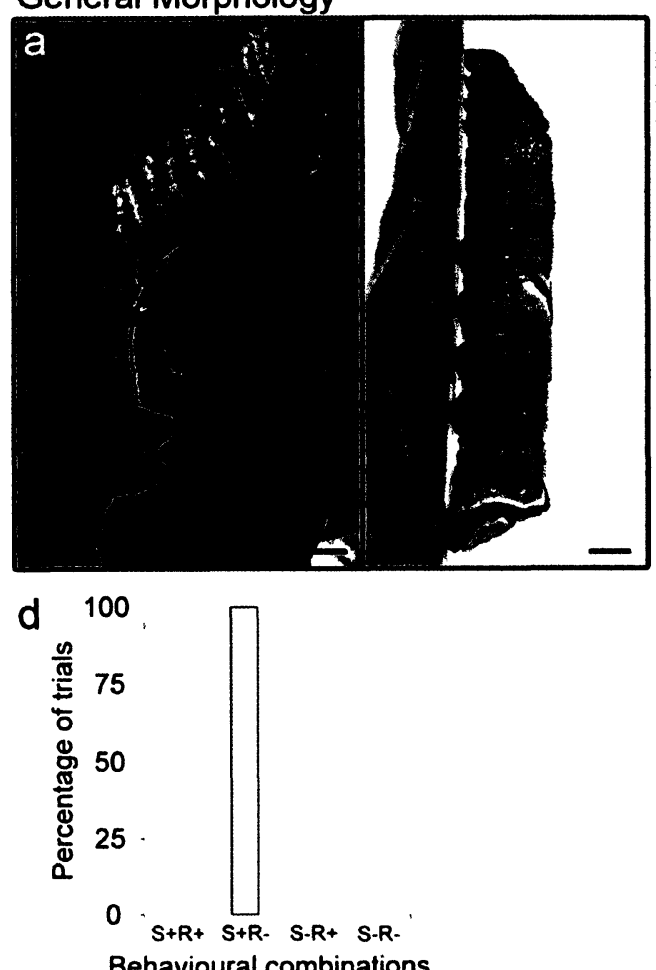

Defensive Behaviours

\begin{tabular}{|ll|}
\hline Regurgitation & $\square$ Biting \\
$\square$ Chemical defense & $\square$ Curling-in \\
$\square$ Thrashing & $\square$ Curling up \\
$\square$ Major thrashing & $\square$ Twitching \\
$\square$ Directed thrashing & $\square$ Dropping away \\
\hline
\end{tabular}

Figure 5.53 Pachygonidia drucei. General morphological features of a late instar caterpillar. (a) Dorsal view of the caterpillar on its hostplant, scale bar $1 \mathrm{~cm}$. (b) Lateral view of a fifth instar caterpillar, scale bar $0.5 \mathrm{~cm}$. (c) List of defensive behaviours observed upon attack and when handled. (d) Histogram showing the relationship between sound production (S) and regurgitation (R) during the first 5 pinches of an attack $(n=1)$. Image in (a) by Jayne Yack, (b) by Alan Fleming. 
a
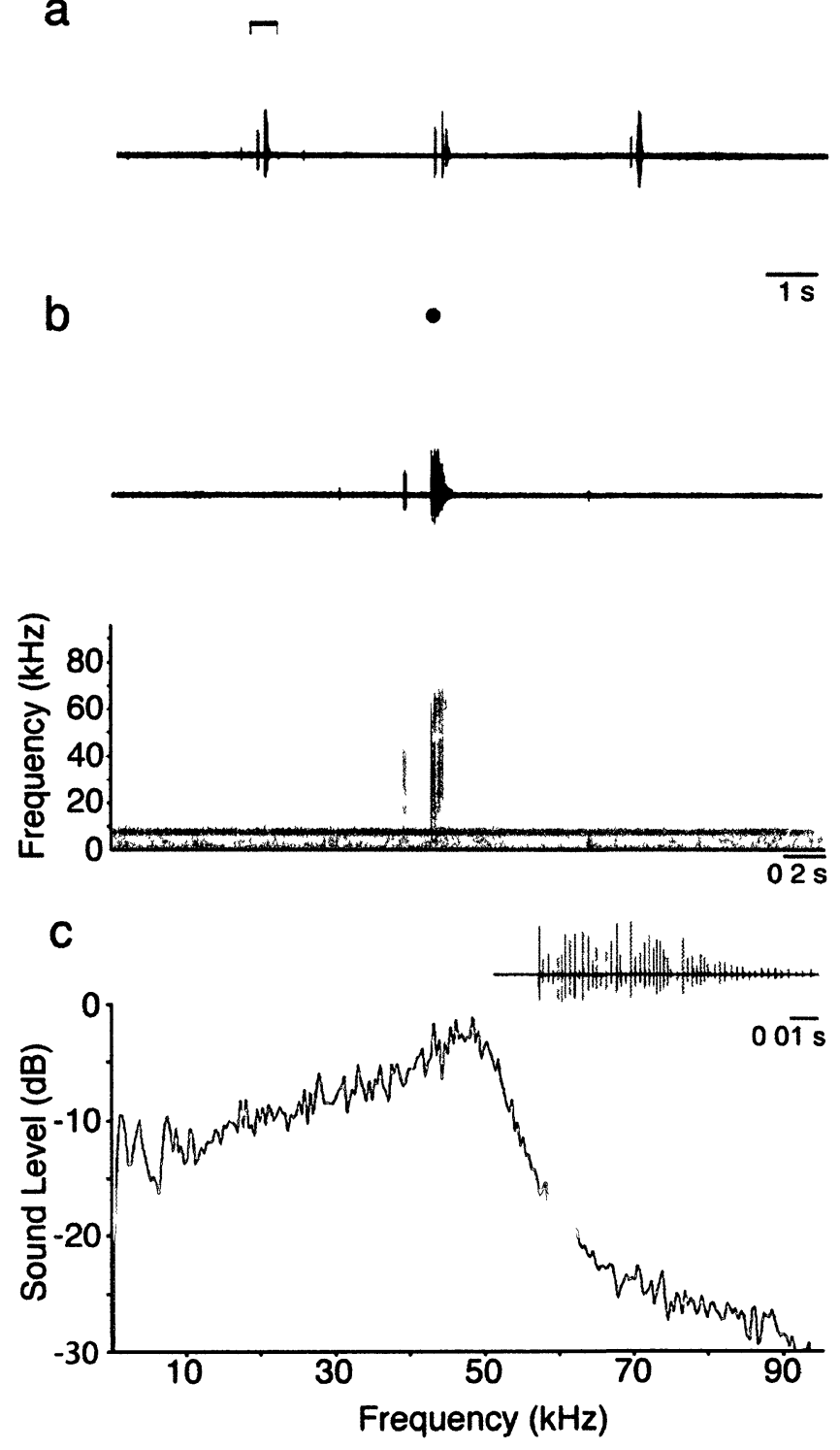

Figure 5.54 Sounds produced by late instar $\boldsymbol{P}$. drucei caterpillars. (a) Oscillogram of rasps following attack. (b) Time expansion of bracket in (a). The accompanying spectrogram shows the frequency distribution of each rasp. (c) Power spectrum taken from one individual. Further expanded time scale of rasp (dot in b) is inset showing the multiple components. 
Sphecodina abbottii (Swainson), the Abbott's sphinx moth, Fig 5.55

The Abbott's sphinx is a new world moth found throughout eastern North America (Wagner 2005, Tuttle 2007). Larvae of this species feed on Ampelopsis (Ampelopsis sp.), grape (Vitis sp.) and Virginia creeper (Parthenocissus quinquefolia), all members of the Vitaceae family (Wagner 2005, Tuttle 2007).

Morphology: The final instar of this species appears in two cryptic morphs (Wagner 2005, Tuttle 2007). The brown morph resembles the wooden bark of the vine and the green saddled morph is said to resemble bunches of grapes (Heinrich 1979, Wagner 2005, Tuttle 2007). Both of the morphs have lost their characteristic horn and replaced it with a convincing 'eye' (Wagner 2005). I have only encountered the brown morph of these caterpillars (Fig 5.55 b) during the course of my studies, and in very limited numbers $(n=4)$. The fifth instar of this morph was approximately $7-7.5 \mathrm{~cm}$ in length, had a rounded head with an area of $20.97 \mathrm{~mm}^{2}$ (Fig $5.55 \mathrm{c}$ ), and ridged and serrated mandibles (Fig $5.55 \mathrm{e}-\mathrm{h}$ ).

Behavioural trials (Fig 5.55 i): Abbott's sphinx caterpillars rest along the stem or branch of their food plant (Fig $5.55 \mathrm{~b}$ ). They remain in this position when lightly disturbed, only responding when stimulation is quite strong (personal observation, Heinrich 1979). When attacked anteriorly the caterpillar responded with sound production (rasping and clicking) and by thrashing in a wide arc away from the forceps and curling upward, and then, doing the same thing in the opposite direction (Fig $5.55 \mathrm{~d}$ ). When attacked posteriorly the same behaviours were observed but the initial thrash was towards the site of attack instead of away. When handled, these caterpillars would deliver a strong bite whenever possible. As the larva became pre-pupal it would more readily 
drop from its food plant when attacked and occasionally adopt a snake-like posture if further harassed on the ground, whereby it would curl-up and raise its posterior end off the ground displaying its 'eye'. If provoked after adopting this posture the caterpillar would flip its body from side to side while producing sound. Thrashing, sound production and a snake-like posture during the wandering phase have also been noted by other authors (e.g. Heinrich 1979, Wagner 2005).

Acoustic: The sounds produced by these caterpillars have been described as 'cracklingrasping'and squeaking in anecdotal reports (Heinrich 1979, Wagner 2005).

Sound characteristics (Table 5.3): The acoustic signals produced by $S$. abbottii consist of trains of sounds made up of two distinct components, a group of rasps followed by a series of quiet clicks (Fig $5.56 \mathrm{a}, \mathrm{b}$ ). Trains were on average $6906 \pm 3732 \mathrm{~ms}$ in duration, with $7-36$ sounds, including both rasps and clicks ( $n=8$ trains from 2 animals). Rasps were $82.23 \pm 24.34 \mathrm{~ms}$ in duration and contained 1-30 components, while clicks were $0.74 \pm 0.74 \mathrm{~ms}$ in duration and contained 1-3 component $(\mathrm{n}=165$ sounds). Rasps were broadband (mean Q3 $=9.49 \pm 3.17, \mathrm{Q} 10=0.95 \pm 0.23, \mathrm{n}=9$ rasps from 2 animals $)$ and had a mean dominant frequency of $33.03 \pm 15.24 \mathrm{kHz}$. Clicks were more narrowband (mean $\mathrm{Q} 3=9.16 \pm 4.48, \mathrm{Q} 10=3.17 \pm 2.14, \mathrm{n}=9$ rasps from 2 animals) with a dominant frequency of $24.86 \pm 14.48 \mathrm{kHz}$ (Fig $5.56 \mathrm{c}$ ).

Mechanism: Video analysis revealed that the rasp portion of the signal is not produced by the mandibles, as has previously been suggested (Heinrich 1979). The caterpillar first opens its mandibles maximally and then produces the signal. To date, the exact mechanism remains unknown but it may be produced by the movement of air past a flap in the foregut (see Part B). Clicks, however, are likely produced by the mandibles, with 
one contacting the other as they are closed (as in other species described in this thesis). Clicks were never produced when the mandibles of the caterpillar were magnified so the possibility cannot be ruled out.

Behaviours associated with sound production and proposed function: The rasp portion of the signal occurred during the winding thrash, while the clicking occurred while the animal was curled-up. The lack of regurgitation or other overt chemical defence in these species (Fig $5.55 \mathrm{j}$ ) suggests the sounds may function as part of a startle display. However, due to the nasty bite these caterpillars are capable of inflicting, sounds may also function as a warning. Though presumably palatable, when two brown morphs were placed on bark within 1-2 m of the nest of a Red-eyed Vireo (Vireo olivaceus) they were completely ignored (Heinrich 1979). 


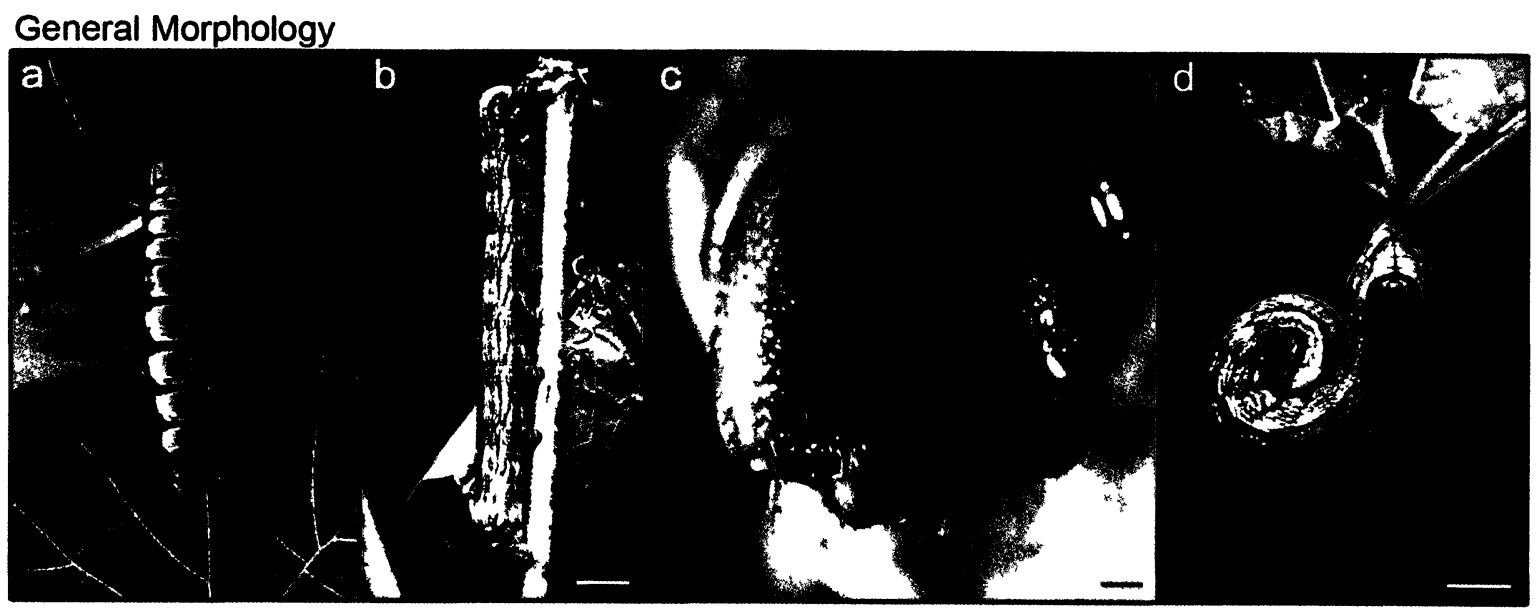

Mandibles

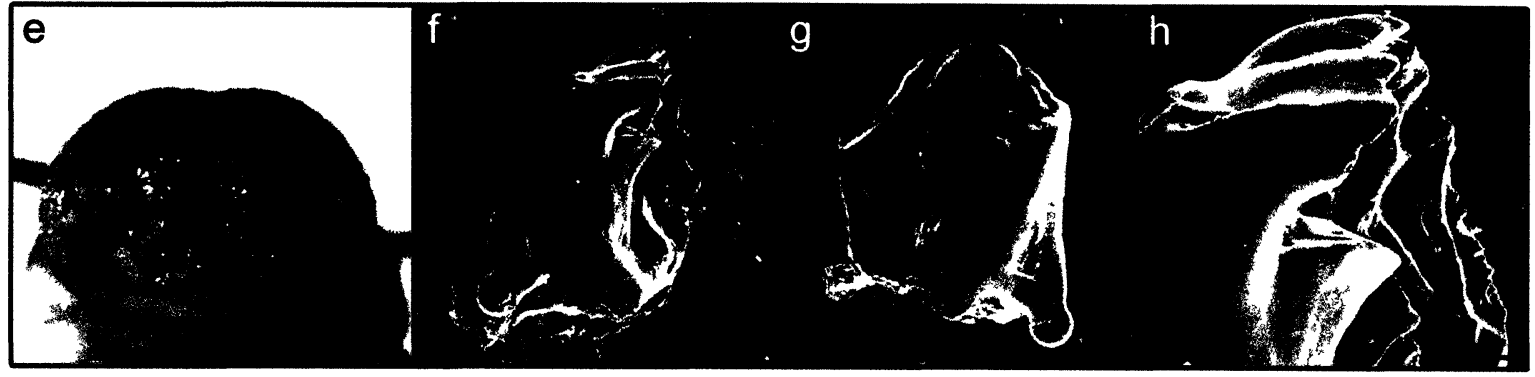

Defensive Behaviours

\begin{tabular}{|ll|}
\hline i $\square$ Sound Production & $\square$ Bitıng \\
$\square$ Regurgitation & $\square$ Curlıng-in \\
$\square$ Chemical defense & $\square$ Curlıng up \\
$\square$ Thrashing & $\square$ Twitching \\
$\square$ Major thrashıng & $\square$ Walk away \\
$\square$ Directed thrashing & $\square$ Dropping \\
\hline
\end{tabular}

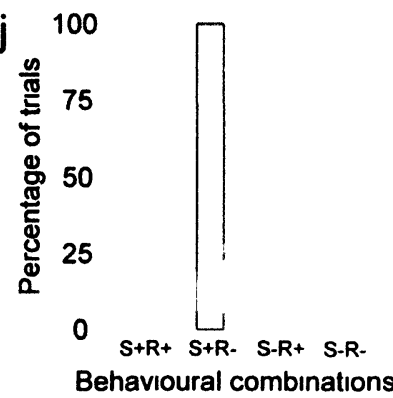

Figure 5.55 Sphecodina abbottii, the Abbott's sphinx. General morphological features of a late instar caterpillar. (a) Lateral view of a late instar larva on its hostplant, scale bar $0.5 \mathrm{~cm}$. (b) Lateral view of a fifth instar caterpillar, scale bar $0.75 \mathrm{~cm}$. (c) Anterior view of the head capsule of a fifth instar, scale bar $0.5 \mathrm{~mm}$. (d) A late instar caterpillar in curled-up position, scale bar 0.75 $\mathrm{cm}$. Mandibles of a late instar caterpillar. (e) The mandibles of a late instar in closed position, scale bar $0.5 \mathrm{~mm}$; (f) right mandible, ventral-anterior view, scale bar $0.25 \mathrm{~mm}$; (g) left mandible, inner face, scale bar $0.25 \mathrm{~mm}$; (h) close up of anterior edge of right mandible, scale bar $0.1 \mathrm{~mm}$. (i) List of defensive behaviours observed upon attack and when handled. (j) Histogram showing the relationship between sound production (S) and regurgitation $(R)$ during the first 5 pinches of an attack $(n=3)$. All images taken by Veronica Bura, except (a) by Freda Holmes (http://bugguide.net/node/view/206760/bgimage). 
a

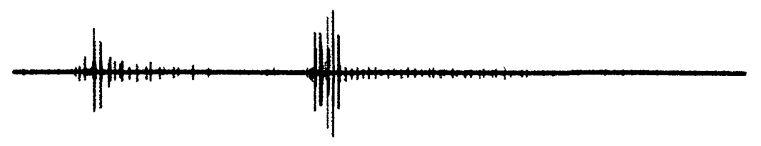

b $\overline{25}$
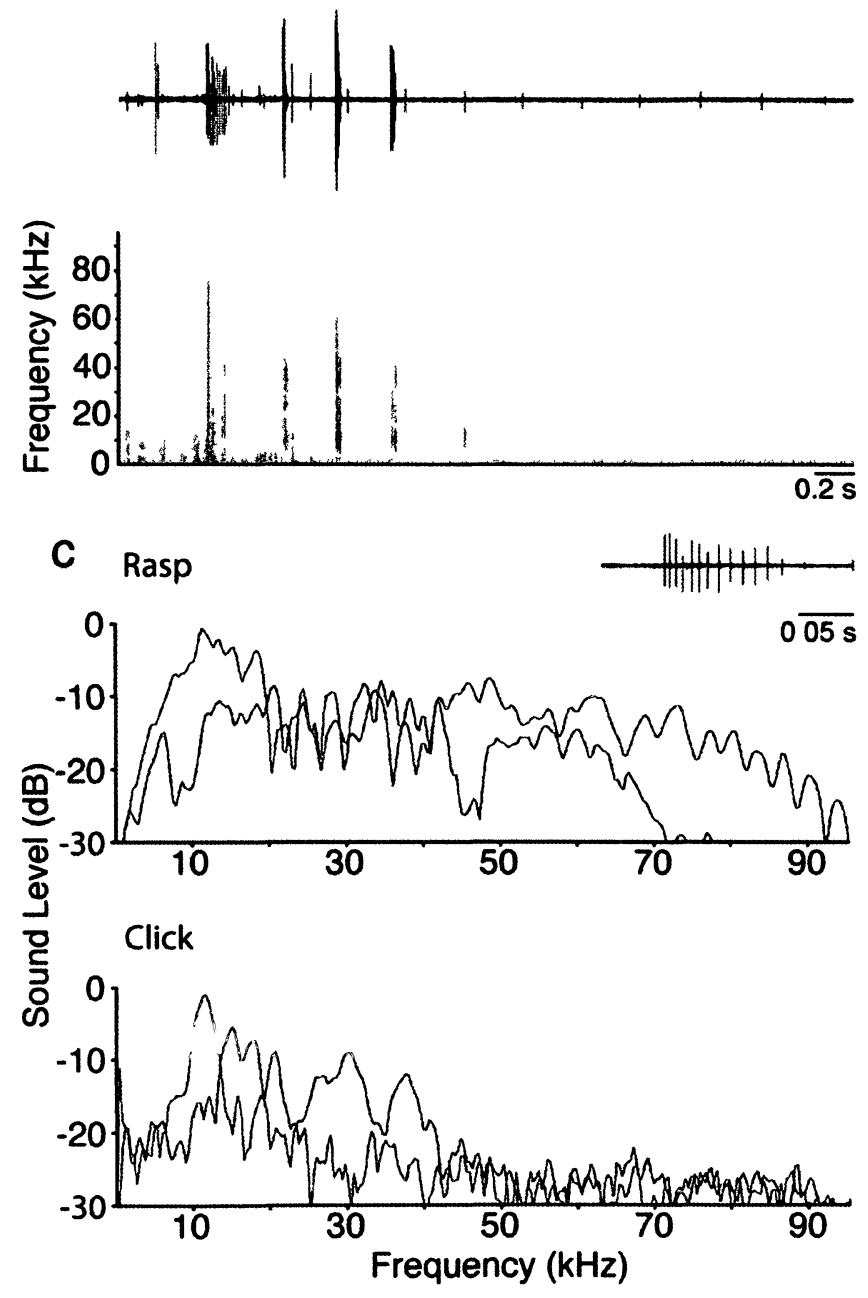

Figure 5.56 Sounds produced by late instar $S$. abbottii caterpillars. (a) Oscillogram of rasps and clicks following attack. (b) Time expansion of bracket in (a). The accompanying spectrogram shows the frequency distribution of each rasp. (c) Power spectra taken from two individuals. Further expanded time scale of rasp (dot in $b$ ) is inset showing the multiple components. 
Xylophanes anubus (Cramer), Fig 5.57

Xylophanes anubus is a new world moth found from southern Mexico through Central America and south to Argentina (Oehkle 2009, Savela 2009). Caterpillars feed on members of the Rubiaceae family, mainly Psychotria sp. (17 species) (Janzen and Hallwachs 2009).

Morphology: Late instar caterpillars can be green or brown (Fig $5.57 \mathrm{a}, \mathrm{b}$ ) (Janzen and Hallwachs 2009). They possess a pair of thoracic eyespots on their dorsal surface and are capable of making them appear more prominent by retracting their head and thorax into their abdomen (personal observation). The specimen I tested was $4.6 \mathrm{~cm}$ in length. Behavioural Trials (Fig 5.57 c): Late instar $X$. anubus larvae rest at the base of their hostplant or under a branch when not feeding (Janzen 1984). Posterior attacks caused the penultimate instar caterpillar to thrash towards the site of attack and begin twitching, subsequently regurgitating a lot and trying to get it on the attacking forceps. Between pinches, the caterpillar ran up the mid-vein of the leaf towards the petiole, attempting to leave the experimental area. When attacked anteriorly the caterpillar curled-in by retracting its head and thorax into its abdomen and thrashed away from the pinch. Pinches were then concentrated on the eye-spots and little to no response was observed other than the caterpillar attempting to leave the area. No sound production was observed. Curling-in in this species may enhance the eyespots on its thorax, potentially mimicking a snake, and likely functions as its main defence. 
General Morphology

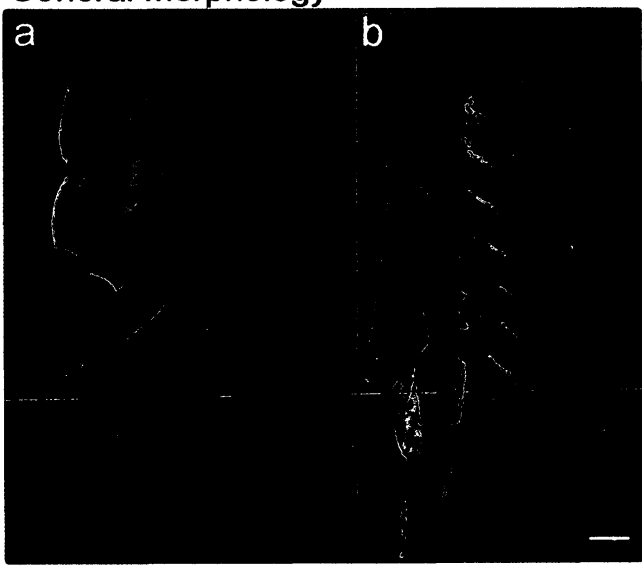

Defensive Behaviours

\begin{tabular}{|ll|}
\hline$\square$ Sound Production & $\square$ Biting \\
$\square$ Regurgitation & $\square$ Curling-in \\
$\square$ Thrashing & $\square$ Curling up \\
$\square$ Major thrashing & $\square$ Twitching \\
$\square$ Directed thrashing & $\square$ Walk away \\
\hline
\end{tabular}

Figure 5.57 Xylophanes anubus. General morphological features of a late instar caterpillar. (a) Dorsal view of a late instar caterpillar on its hostplant, scale bar $1 \mathrm{~cm}$. (b) Lateral view of a fifth instar caterpillar, scale bar $0.5 \mathrm{~cm}$. (c) List of defensive behaviours observed upon attack and when handled. Image in (a) by Jayne Yack, (b) by Dan Janzen. 
Xylophanes tyndarus (Boisduval), Fig 5.58

Xylophanes tyndarus is a new world moth that is found from southern Mexico, through Central America to Brazil, Bolivia and Paraguay (Oehkle 2009, Savela 2009). Caterpillars feed on Faramea occidentalis, a member of the Rubiaceae family (Janzen and Hallwachs 2009).

Morphology: Final instar caterpillars of $X$. tyndarus are brown and have a pair of black thoracic eyespots on their dorsal surface (Fig $5.58 \mathrm{a}, \mathrm{b})$. The two specimens I tested were similar in length, around $6.3 \mathrm{~cm}$.

Behavioural Trials (Fig 5.58 c): Prior to being attacked, the fifth instar larva was resting on the stem of its piece of hostplant (Fig $5.58 \mathrm{a}, \mathrm{b})$. When attacked posteriorly the caterpillar thrashed towards the site of attack and a little from side to side, and eventually it dropped from the hostplant. Twitching was also observed in one animal. Anterior attacks caused extreme curling-in with head and thorax retracted into the abdomen and thrashing away from the attack. No sound production was observed. The eye-spots on the dorsal side of the caterpillar, and accompanying behaviour to exaggerate them, likely function as the main defence in this species and create the appearance of a small snake. 


\section{General Morphology}

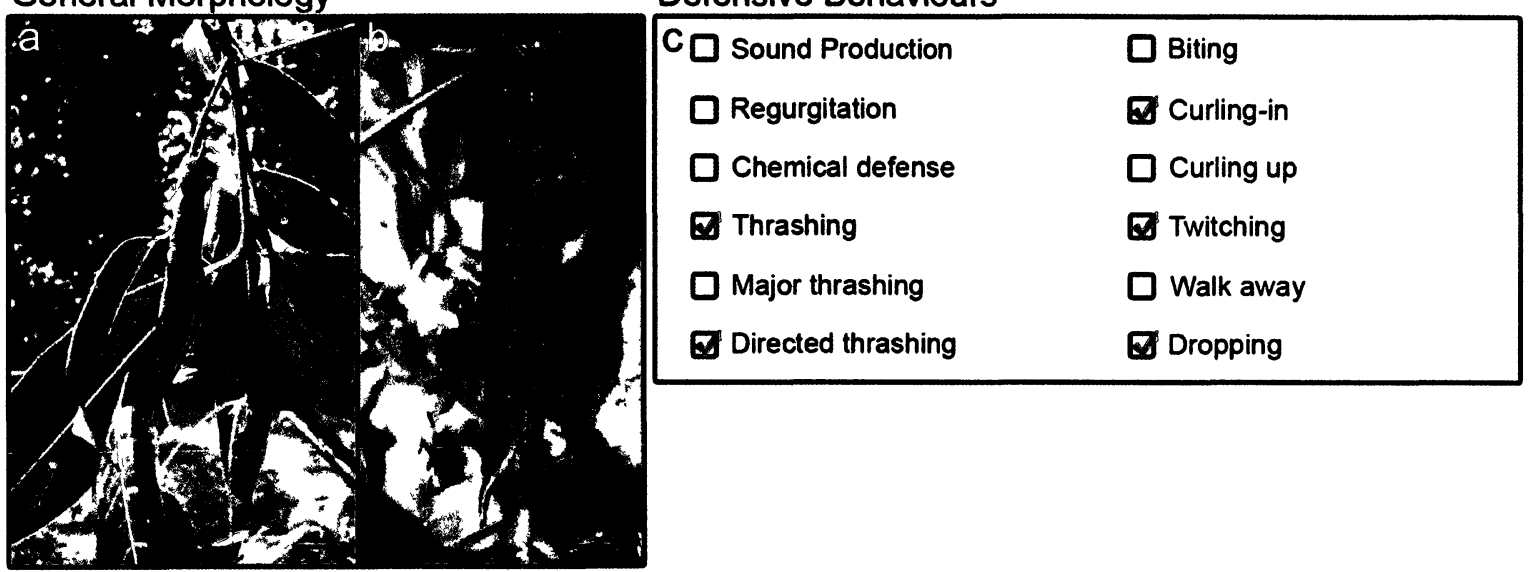

Figure 5.58 Xylophanes tyndarus. General morphological features of a late instar caterpillar. (a) Dorsal view of the caterpillar on its hostplant, scale bar $1 \mathrm{~cm}$. (b) Lateral view of a fifth instar caterpillar, scale bar $0.75 \mathrm{~cm}$. (c) List of defensive behaviours observed upon attack and when handled. Image in (a) by Veronica Bura, (b) by Alan Fleming. 


\section{OUTGROUPS}

The species that follow were tested because they were large caterpillars $(>3 \mathrm{~cm}$ in length) and were readily available.

\section{SUPERFAMILY: LASIOCAMPOIDEA}

\section{FAMILY: LASIOCAMPIDAE}

Many lasiocampid caterpillars have long, thin setae along the side of their bodies and near the head (Wagner 2005). 'Hairy' caterpillars are protected against some predators (discussed earlier).

Euglyphis sp., Fig 5.59

This unnamed species of Euglyphis is a moth found in Costa Rica (Janzen and Hallwachs 2009). This particular specimen was found eating Cinnamomum brenesii (Lauraceae) (Janzen and Hallwachs 2009).

Morphology: The Euglyphis sp. larva was covered in long setae, had red and black patches along its dorsal side and a bright orange head (Fig 5.59 a,b). At the time of testing this specimen was $6.2 \mathrm{~cm}$ in length (Janzen and Hallwachs 2009).

Behavioural Trials (Fig 5.59 c): Prior to experimentation the caterpillar was on the underside of a leaf of hostplant. When attacked posteriorly the caterpillar began walking away from the attacking forceps and dropped off the plant after the third pinch. Anterior attacks caused the larva to turn away and attempt to avoid the pinches and it eventually dropped off the plant. When the caterpillar was picked up off the ground with the forceps it went limp, as if mimicking illness. This specimen also dropped from the leaf as it was being positioned for photographs. No sound production was observed. 
General Morphology

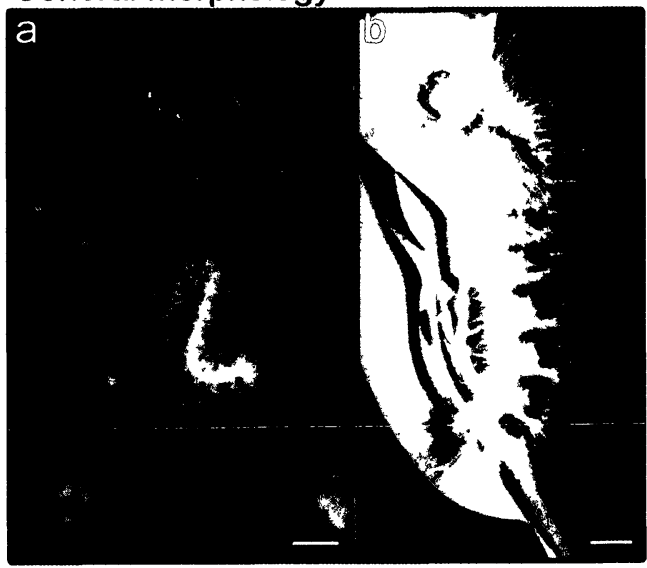

Defensive Behaviours

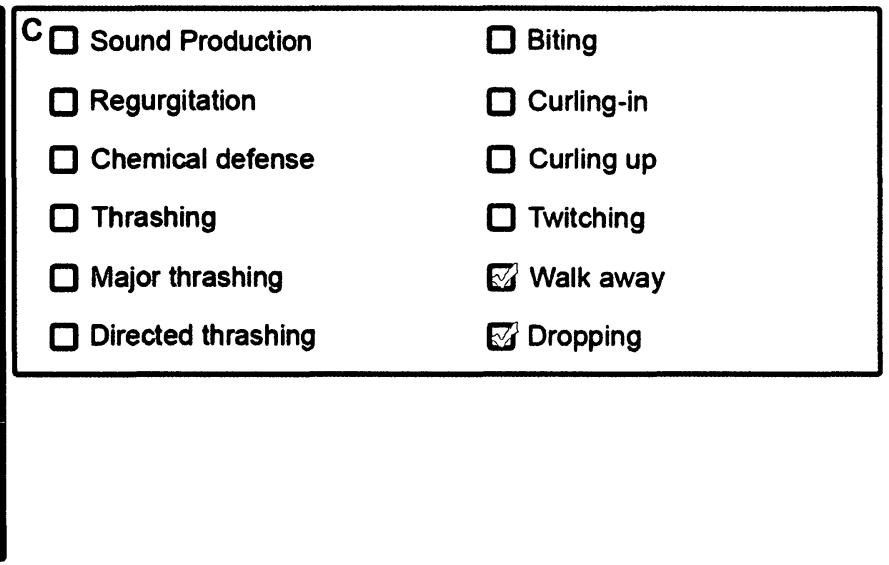

Figure 5.59 Euglyphis sp. General morphological features of a late instar caterpillar. (a) Dorsal view of the caterpillar on its hosplant, scale bar $1 \mathrm{~cm}$. (b) Lateral view of a final instar caterpillar, scale bar $0.5 \mathrm{~cm}$. (c) List of defensive behaviours observed upon attack and when handled. Image in (a) by Jayne Yack, (b) by Dan Janzen. 


\section{SUPERFAMILY: NOCTUOIDEA}

\section{FAMILY: NOCTUIDAE}

This is the largest family of Lepidoptera (over 35000 species) and species exhibit a large variety of defensive behaviours and morphologies (Wagner 2005).

Acanthodica sinuilinea Prout, Fig 5.60

Acanthodica sinuilinea is a new world moth found in Costa Rica and Peru (Savela 2009, Janzen and Hallwachs 2009). Caterpillars of this species feed on Heteropterys obovata of the Malpighiaceae family (Janzen and Hallwachs 2009).

Morphology: The final instar caterpillar of $A$. sinuilinea was brown, slender and resembled a twig (Fig $5.60 \mathrm{a}, \mathrm{b}$ ). The specimen I tested was $5.8 \mathrm{~cm}$ in length at the time of experimentation.

Behavioural Trials (Fig 5.60 c): The larva was resting on the woody portion of its hostplant prior to experimentation. When attacked posteriorly the caterpillar thrashed towards the site of attack and raised its anal prolegs off the substrate, possibly to make it seem as if this was its head. Subsequent pinches elicited the same behaviours and the caterpillar quickly moved away from the site of attack. As the caterpillar was picked up and placed back on the sprig it either regurgitated or released fluid from its posterior end in response. No sound production was observed. 
General Morphology

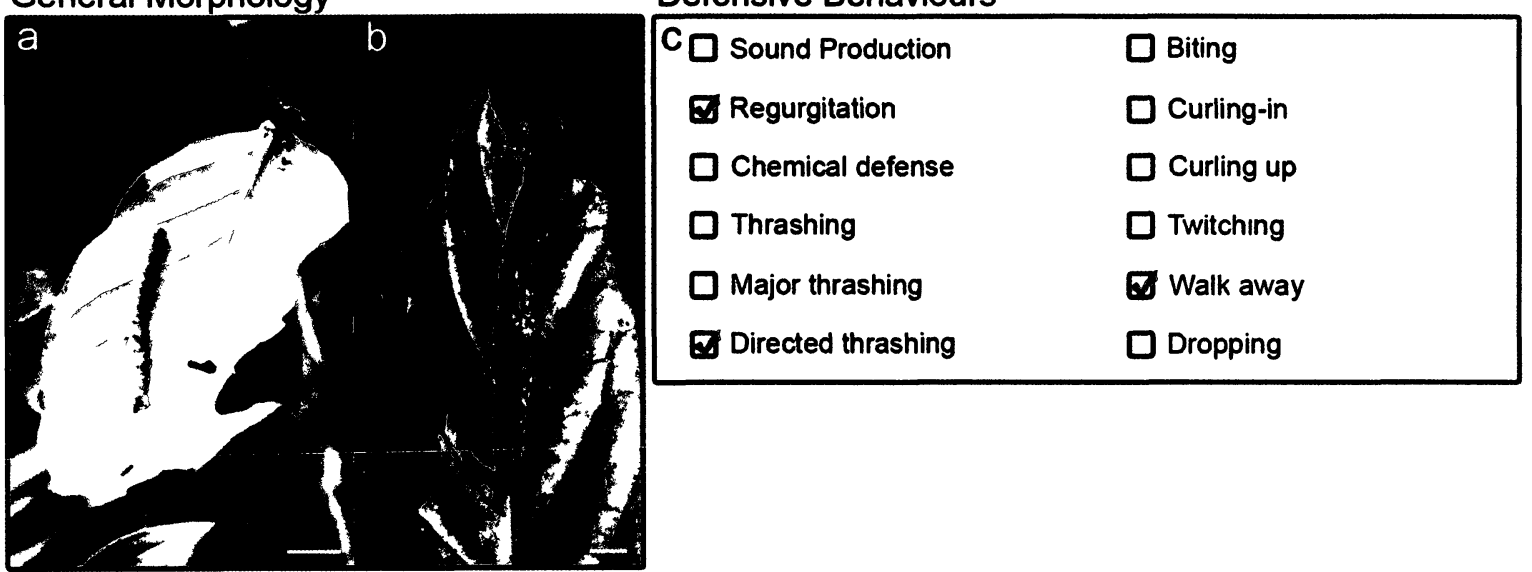

Figure 5.60 Acanthodica sinuilinea. General morphological features of a late instar caterpillar. (a) Dorsal view of a late instar caterpillar on its hostplant, scale bar $2 \mathrm{~cm}$. (b) Dorso-lateral view of a final instar caterpillar, scale bar $0.5 \mathrm{~cm}$. (c) List of defensive behaviours observed upon attack and when handled. Image in (a) by Jayne Yack, (b) by Dan Janzen. 


\section{Unknown noctuid, Fig 5.61}

This unnamed member of the family Noctuidae is found in Costa Rica whose caterpillars have been reported to feed on Dioscorea urophylla from the Dioscoreaceae family (Janzen and Hallwachs 2009).

Morphology: This caterpillar was pale green, had a pair of white lateral stripes, a yellow head and yellow spots on its posterior end and around its spiracles (Fig $5.61 \mathrm{a}, \mathrm{b}$ ). This specimen was $4.5 \mathrm{~cm}$ in length.

Behavioural Trials (Fig 5.61 c): Prior to experimentation the caterpillar was resting on the upper surface of a leaf of its hostplant. Posterior attacks caused the larva to thrash towards the site of attack and scrape its mandibles against the leaf surface while regurgitating, which was slightly audible. This motion was repeated up to three times per attack. After prolonged attacks the caterpillar exhibited a strange twitching-slow side to side thrashing motion and started to walk away from the encounter. No sound production was observed. 
General Morphology

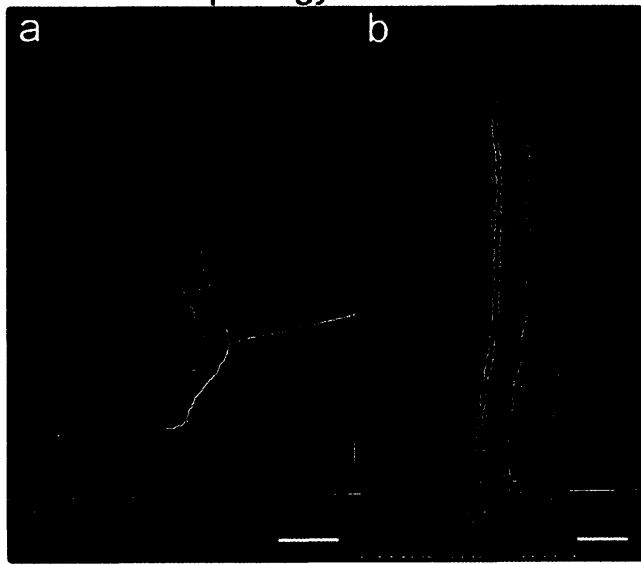

Defensive Behaviours

\begin{tabular}{|ll|}
\hline$\square$ Sound Production & $\square$ Biting \\
$\square$ Regurgitation & $\square$ Curling-in \\
$\square$ Chemical defense & $\square$ Curling up \\
$\square$ Thrashing & $\square$ Twitching \\
$\square$ Major thrashing & $\square$ Walk away \\
$\square$ Directed thrashing & $\square$ Dropping \\
\hline
\end{tabular}

Figure 5.61 Unknown noctuid. General morphological features of a late instar caterpillar. (a) Dorsal view of a late instar caterpillar on its hostplant, scale bar $1 \mathrm{~cm}$. (b) Lateral view of a final instar caterpillar, scale bar $0.5 \mathrm{~cm}$. (c) List of defensive behaviours observed upon attack and when handled. Image in (a) by Jayne Yack, (b) by Alan Fleming. 


\section{FAMILY: NOTODONTIDAE}

At least some species in this family are chemically defended and will either regurgitate or shoot acid from a neck gland when disturbed (Wagner 2005).

Crinodes besckei (Hübner), Fig 5.62

Crinodes besckei is a new world moth found in Mexico, Central America and Brazil (Savela 2009). Caterpillars feed on species in the genus Gouania from the Rhamnaceae family (Janzen and Hallwachs 2009).

Morphology: Late instar caterpillars of $C$. besckcei can be green, brown or pink, highly patterned and have dark patches on their ventral side at the base of their third set of thoracic legs (Fig $5.62 \mathrm{a}, \mathrm{b}$, Miller et al. 2006). This specimen was about $5 \mathrm{~cm}$ in length. Behavioural Trials (Fig 5.62 c): Prior to experimentation the caterpillar was resting in its characteristic ' $\mathrm{C}$ ' shaped posture (Fig 5.62 a). When attacked posteriorly the caterpillar brought its anterior and posterior ends closer together and immediately regurgitated. The same behaviours were observed for anterior pinches. No sound production was observed. This species of caterpillar has also been observed to drop from its hostplant immediately if the vine is even touched, sometimes with a fatal result (Miller et al. 2006). 
General Morphology

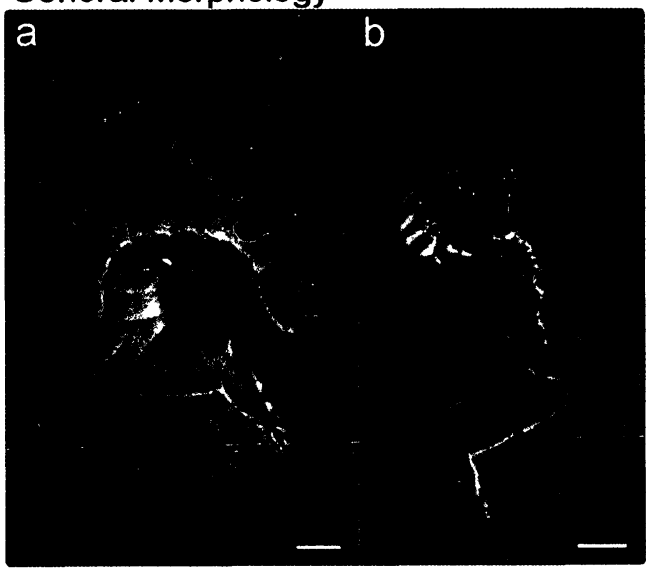

Defensive Behaviours

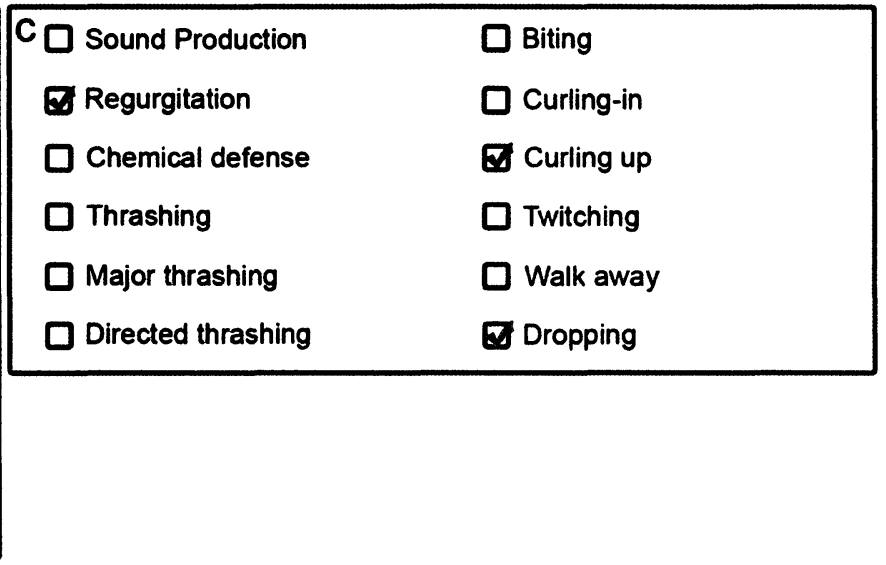

Figure 5.62 Crinodes besckei. General morphological features of a late instar caterpillar. (a) Lateral view of a pinkish brown morph showing its curling-up behaviour, scale bar $0.5 \mathrm{~cm}$. (b) Lateral view of a green morph, scale bar $0.5 \mathrm{~cm}$. (c) List of defensive behaviours observed upon attack and when handled. All images were taken by Dan Janzen. 
Lirimiris fascis, Fig 5.63

Lirimiris fascis is a new world moth found in Costa Rica (Janzen and Hallwachs 2009). This caterpillar feeds on two species of Malvaceae and Tiliaceae (Janzen and Hallwachs 2009).

Morphology: The final instar of $L$. fascis was brightly coloured, mainly yellow, black and red, had a white patch on its posterior end and had tufts of hair on its anterior and posterior ends (Fig $5.63 \mathrm{a}, \mathrm{b}$ ). This specimen was about $8 \mathrm{~cm}$ in length.

Behavioural Trials (Fig 5.63 c): Prior to being attacked the caterpillar was resting on a leafless portion of its hostplant (Fig $5.63 \mathrm{a}, \mathrm{b})$. When attacked anteriorly the larva thrashed away from the pinch and curled-in. Posterior attacks did not result in much change, as the animal was already in the curled-in position. No sound production was observed. 
General Morphology

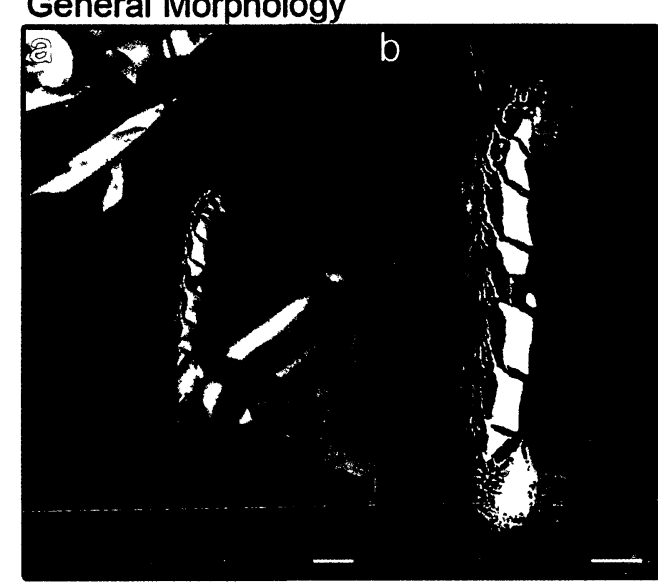

Defensive Behaviours

\begin{tabular}{|ll|}
\hline$\square$ Sound Production & $\square$ Biting \\
$\square$ Regurgitation & $\square$ Curling-in \\
$\square$ Chemical defense & $\square$ Curling up \\
$\square$ Thrashing & $\square$ Twitching \\
$\square$ Major thrashing & $\square$ Walk away \\
$\square$ Directed thrashing & $\square$ Dropping \\
\hline
\end{tabular}

Figure 5.63 Lirimiris fascis. General morphological features of a late instar caterpillar. (a) Dorso-lateral view of a late instar caterpillar on its hostplant, scale bar $1.5 \mathrm{~cm}$. (b) Dorso-lateral view of a fifth instar caterpillar, scale bar $1 \mathrm{~cm}$. (c) List of defensive behaviours observed upon attack and when handled. Image in (a) by Jayne Yack, (b) by Alan Fleming. 


\section{Lirimiris guatemalensis Rothschild, Fig 5.64}

Lirimiris guatemalensis is a new world moth found in Costa Rica (Janzen and Hallwachs 2009). The caterpillar of this species feeds on various species of plants in the families Sterculiaceae and Tiliaceae (Janzen and Hallwachs 2009).

Morphology: The final instar caterpillar of $L$. guatemalensis was yellow and black with a red head and a red bulb on its posterior end (Fig $5.64 \mathrm{a}, \mathrm{b}$ ). This specimen was $4.7 \mathrm{~cm}$ in length at the time of experimentation.

Behavioural Trials (Fig 5.64 c): Prior to experimentation the caterpillar was resting on the underside of a leaf of hostplant, along the mid-vein. This species did not respond much to either attack, if the larva was moving prior to being pinched it stopped the movement and some anterior attacks resulted a thrash away from the pinch. No sound production was observed. 
General Morphology

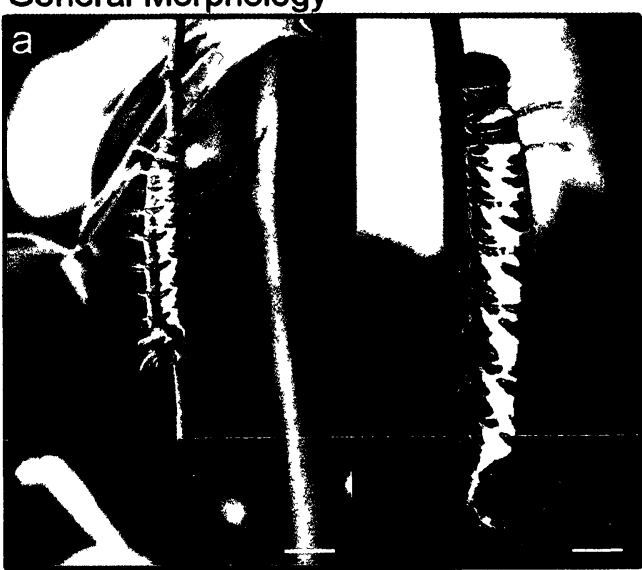

Defensive Behaviours

\begin{tabular}{|ll|}
\hline$\square$ Sound Production & $\square$ Biting \\
$\square$ Regurgitation & $\square$ Curling-in \\
$\square$ Chemical defense & $\square$ Curling up \\
$\square$ Thrashing & $\square$ Twitching \\
$\square$ Major thrashing & $\square$ Walk away \\
$\square$ Directed thrashing & $\square$ Dropping \\
\hline
\end{tabular}

Figure 5.64 Lirimiris guatemalensis. General morphological features of a late instar caterpillar. (a) Dorsal view of a late instar caterpillar on its hosplant, scale bar $1 \mathrm{~cm}$. (b) Dorso-lateral view of a final instar caterpillar, scale bar $0.5 \mathrm{~cm}$. (c) List of defensive behaviours observed upon attack and when handled. Image in (a) by Jayne Yack, (b) by Alan Fleming. 


\section{FAMILY: NYMPHALIDAE}

This family of quite large (6000 species) and contains three subfamilies that have caterpillars adorned with scoli (Wagner 2005). In at least one of these subfamilies the scoli are completely harmless (Wagner 2005) and may act as a bluff.

Antirrhea lindigii Felder, Fig 5.65

Antirrhea lindigii (current accepted name Antirrhea miltiades, Lepindex) is a new world butterfly found throughout Central America and much of northern South America (Savela 2009). Caterpillars feed on various members of the palm family Arecaceae as well as Calathea lutea of the Marantaceae in Costa Rica (Janzens and Hallwachs 2009). Morphology: Final instar caterpillars of $A$. lindigii colourful, with patterns of yellow and orange along their dorsal margin (Fig $5.65 \mathrm{a}, \mathrm{b})$. Their bodies are covered in hair elsewhere and they have two long setae on their posterior end (resembling furcula). The specimen I tested was about $3.2 \mathrm{~cm}$ in length.

Behavioural Trials (Fig 5.65 c): The larva was resting on the leaf of hostplant prior to experimentation (Fig 5.65 a). When attacked anteriorly the caterpillar would fling its long setae forward towards the attacking forceps and move its head quickly from side to side. This side to side movement appeared to produce sounds by contacting the leaf similar to those produced by drepanid species during territorial encounters (Yack et al. 2001, Bowen et al. 2008, Scott et al. 2010a,b). The thoracic legs were also moving rapidly and may have been responsible for the percussive sounds. I believe that the sounds produced by the movement of the head or legs would likely be transmitted as vibrations. The thrashing behaviour of the posterior setae has been shown in other species of this genus to deter attacking parastitoids (Greeney et al. 2009). 
General Morphology

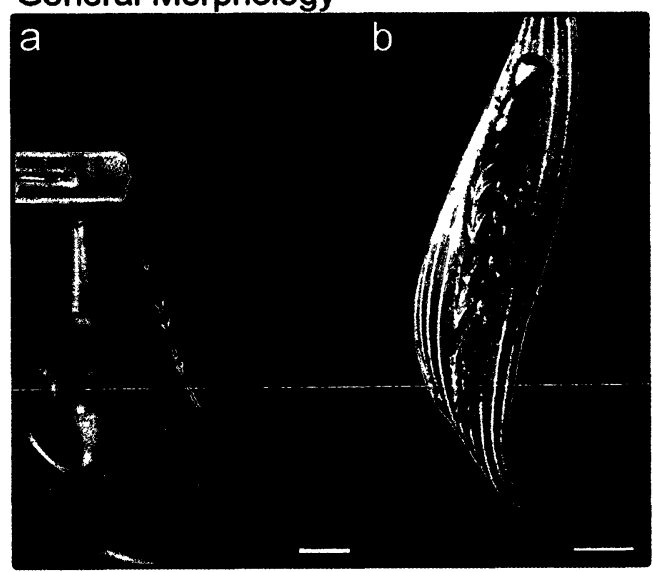

Defensive Behaviours

\begin{tabular}{|ll|}
\hline$\square$ Sound Production & $\square$ Biting \\
$\square$ Regurgitation & $\square$ Curling-in \\
$\square$ Chemical defense & $\square$ Curling up \\
$\square$ Thrashing & $\square$ Twitching \\
$\square$ Major thrashing & $\square$ Walk away \\
$\square$ Directed thrashing & $\square$ Dropping \\
\hline
\end{tabular}

Figure 5.65 Antirrhea lindigii. General morphological features of a late instar caterpillar. (a) Dorsal view of the caterpillar on its hostplant, scale bar $1 \mathrm{~cm}$. (b) Lateral view of a fifth instar caterpillar, scale bar $1 \mathrm{~cm}$. (c) List of defensive behaviours observed upon attack and when handled. Image in (a) by Jayne Yack, (b) by Dan Janzen. 
Morpho amathonte Deyrolle, Fig 5.66

Morpho amathonte is a new world butterfly found throughout Central America and northern South America (Savela 2009). Caterpillars feed on species in the palm (Arecaceae), legume (Fabaceae) and Dichapetalaceae families (Janzen and Hallwachs 2009).

Morphology: The late instar caterpillar of $M$. amathonte was brightly coloured, yellow being the most prominent colour, and had tufts of white hairs along its lateral and dorsal sides (Fig $5.66 \mathrm{a}, \mathrm{b}$ ). The specimen I tested was about $4.5 \mathrm{~cm}$ in length.

Behavioural Trials (Fig 5.66 c): Prior to experimentation the caterpillar was resting on the dorsal surface of its hostplant (Fig $5.66 \mathrm{a}, \mathrm{b}$ ). Anterior attacks caused the larva to slowly thrash away from the pinch, while posterior attacks caused thrashing towards the site of attack. No sound production was observed. 


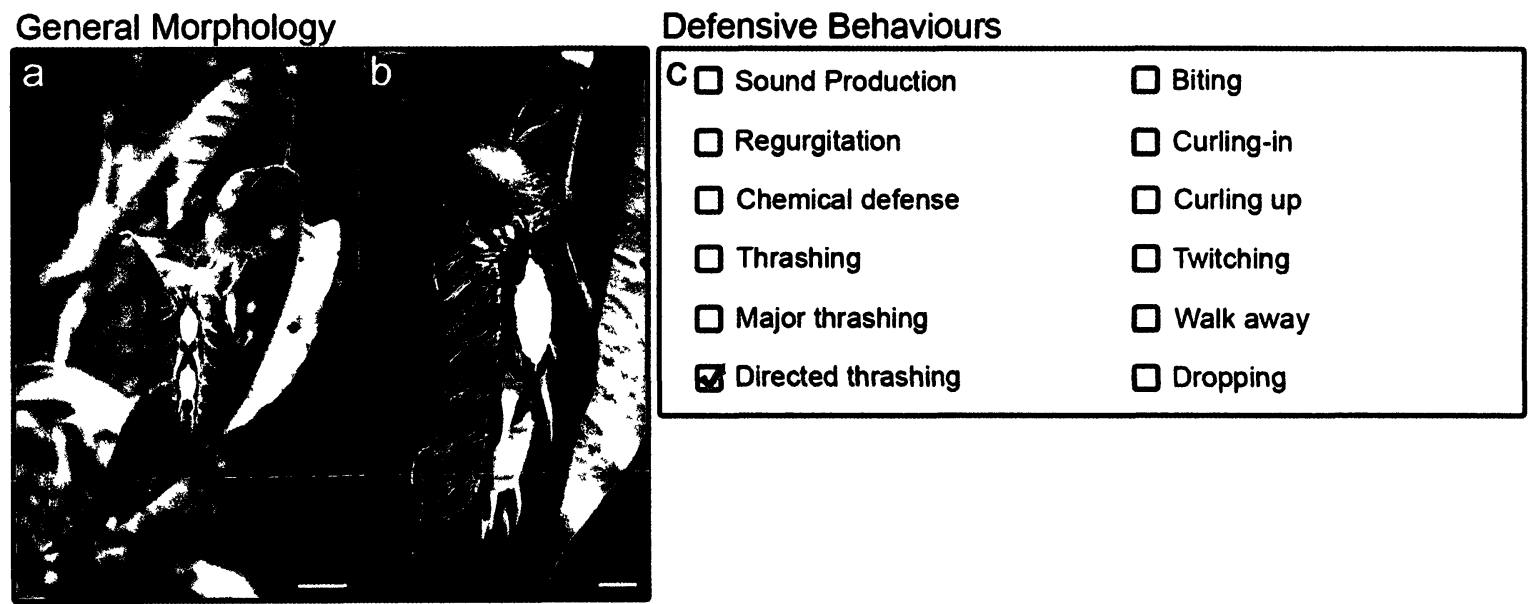

Figure 5.66 Morpho amathonte. General morphological features of a late instar caterpillar. (a) Dorsal view of the caterpillar of its hostplant, scale bar $1.5 \mathrm{~cm}$. (b) Dorso-lateral view of a fifth instar caterpillar, scale bar $0.5 \mathrm{~cm}$. (c) List of defensive behaviours observed upon attack and when handled. Image in (a) by Jayne Yack, (b) by Veronica Bura. 
Danaus sp., Fig 5.67

This unnamed species of Danaus is a butterfly found in Costa Rica (Janzen and Hallwachs 2009). This particular specimen was found eating Solandra grandiflora (Rubiaceae) and the other recorded specimen was found eating Schultesianthus leucanthus (Solanaceae) (Janzen and Hallwachs 2009).

Morphology: The Danaus sp. larva had black and yellow stripes, a small orange band close to the head capsule and two long projections originating at the thorax (Fig $5.67 \mathrm{a}, \mathrm{b}$ ). The specimen I tested measured $3 \mathrm{~cm}$ in length.

Behavioural Trials (Fig 5.67 c): Before the trial began the caterpillar was resting along the midvein on the underside of the leaf (Fig 5.67 a). When attacked posteriorly the fifth instar larva thrashed towards the site of attack, waved the long projections around, releasing a foul smell, and eventually regurgitated. Anterior attacks resulting in immediate regurgitation and thrashing away from the pinch. No sound production was observed. 
General Morphology

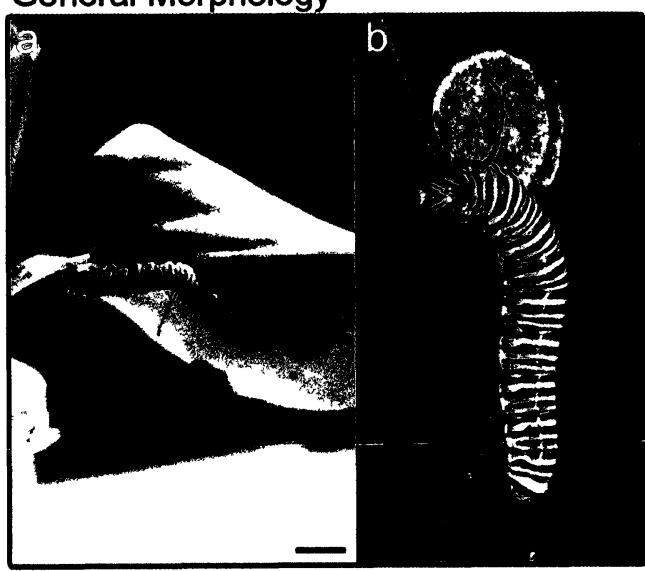

Defensive Behaviours

\begin{tabular}{|ll|}
\hline$\square$ Sound Production & $\square$ Biting \\
$\square$ Regurgitation & $\square$ Curling-in \\
$\square$ Chemical defense & $\square$ Curling up \\
$\square$ Thrashing & $\square$ Twitching \\
$\square$ Major thrashing & $\square$ Walk away \\
$\square$ Directed thrashing & $\square$ Dropping \\
\hline
\end{tabular}

Figure 5.67 Danaus sp. General morphological features of a late instar caterpillar. (a) Lateral view of a fifth instar caterpillar on its hostplant, scale bar $1 \mathrm{~cm}$. (b) Antero-lateral view of a late instar caterpillar, scale bar $0.5 \mathrm{~cm}$. (c) List of defensive behaviours observed upon attack and when handled. Image in (a) by Jayne Yack, (b) by Dan Janzen. 
Smyrna blomfildia (Fabricius), Blomfild's Beauty, Fig 5.68

Blomfild's Beauty is a new world butterfly found from southern Texas through Central America and much of South America (Savela 2009). Caterpillars eat Urera baccifera, Urera caracasana, and Myriocarpa longipes, members of the nettle family (Urticaceae) (Janzen and Hallwachs 2009).

Morphology: The Late instar S. blomfildia caterpillar had a black section along its dorsal side, pale yellowish-white sections along its lateral sides and a red head (Fig $5.68 \mathrm{a}, \mathrm{b}$ ). They are covered in scolus-like projections that are non-urticating (Janzen, personal communication). At the time of experimentation this specimen was about $6.3 \mathrm{~cm}$ in length.

Behavioural Trials (Fig 5.68 c): Prior to experimentation the caterpillar was resting on the underside of the leaves of hostplant (Fig 5.68 a). When attacked anteriorly the caterpillar would thrash away from the pinch and walk away from the encounter. Posterior attacks elicited the same behaviours but the caterpillar thrashed towards the site of attack instead of away. Earlier instars may regurgitate, wriggle or drop from silken threads in response to predation and construct frass chains that have been shown to decrease ant predation (Machado and Freitas 2001). 
General Morphology

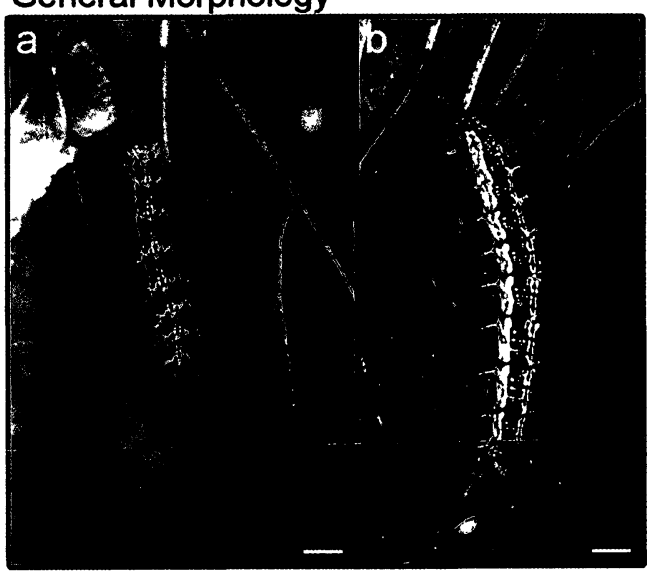

Defensive Behaviours

\begin{tabular}{|ll|}
\hline$\square$ Sound Production & $\square$ Biting \\
$\square$ Regurgitation & $\square$ Curling-in \\
$\square$ Chemical defense & $\square$ Curling up \\
$\square$ Thrashing & $\square$ Twitching \\
$\square$ Major thrashing & $\square$ Walk away \\
$\square$ Directed thrashing & $\square$ Dropping \\
\hline
\end{tabular}

Figure 5.68 Smyrna blomfildia. General morphological features of a late instar caterpillar. (a) Dorsal view of the caterpillar on its hostplant, scale bar $1 \mathrm{~cm}$. (b) Lateral view of a fifth instar caterpillar, scale bar $0.5 \mathrm{~cm}$. (c) List of defensive behaviours observed upon attack and when handled. Image in (a) by Jayne Yack, (b) by Dan Janzen. 


\section{FAMILY: HESPERIIDAE}

Caterpillars in this family construct silken leaf shelters that they rest in during the day (Wagner 2005).

Astraptes creteus crana Evans, Fig 5.69

Astraptes creteues crana is a new world butterfly found in southern Mexico, Guatemala and Costa Rica (Savela 2009, Janzen and Hallwachs 2009). Caterpillars feed on Styrax argenteus, a member of the Styracaceae (Janzen and Hallwachs 2009).

Morphology: The $A$. creteus crana larva was mainly green with a dark red head and a thin "neck" (Fig $5.69 \mathrm{~b}$ ). This specimen measured $4.4 \mathrm{~cm}$ in length at the time of experimentation.

Behavioural Trials (Fig 5.69 c): Prior to the trial the caterpillar was resting in its curled leaf nest (Fig 5.69 a) which was opened in order to attack the larva. When attacked anteriorly the caterpillar had its mandibles wide open ready to bite the attacking forceps. The larva bit any fingers that were close enough to its body. Posterior attacks resulted in the caterpillar biting the forceps. No sound production was observed. 
General Morphology

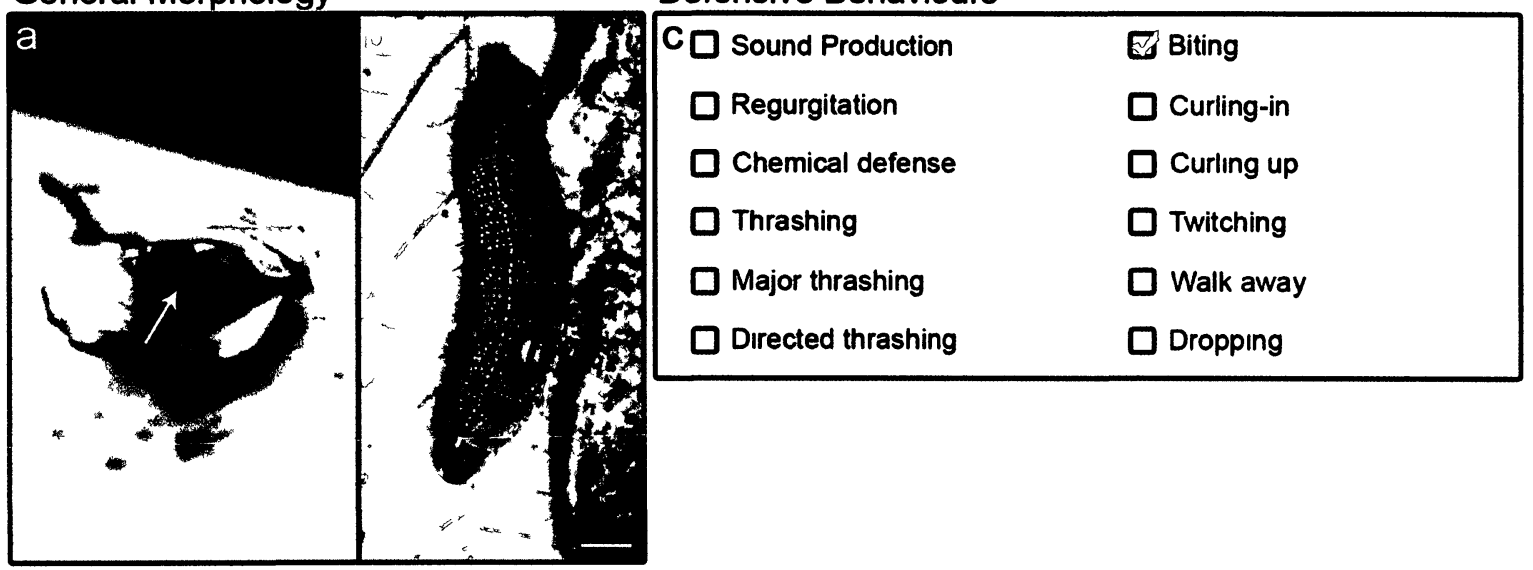

Figure 5.69 Astraptes creteus crana. General morphological features of a late instar caterpillar. (a) A late instar caterpillar inside its rolled leaf nest, the arrow indicates the position of the caterpillar. (b) Dorso-lateral view of a fifth instar larva scale bar $0.5 \mathrm{~cm}$. (c) List of defensive behaviours observed upon amaek amd when hamdled. All images by Jayne Yack. 
Table 5.2 Summary of defensive behaviours and morphological features for Bombycoidea caterpillars observed in this survey. Chemical defence includes regurgitation in this table, and P, A, and F indicate if the sound precedes, accompanies or follows the chemical, respectively. (n/a not available; - not applicable).

\begin{tabular}{|c|c|c|c|c|c|c|c|c|c|}
\hline \multirow[b]{2}{*}{ Family } & \multirow[b]{2}{*}{ Species } & \multirow[b]{2}{*}{ \# tested } & \multicolumn{2}{|c|}{ Sound production } & \multicolumn{3}{|c|}{ Morphology } & \multicolumn{2}{|c|}{ Chemical defence } \\
\hline & & & $\mathrm{Y} / \mathrm{N}$ & Type & $\begin{array}{c}\text { Max body } \\
\text { length }(\mathrm{cm})^{3}\end{array}$ & $\begin{array}{l}\text { Head capsule } \\
\text { area }\left(\mathrm{mm}^{2}\right)\end{array}$ & Mandible type & $\mathrm{Y} / \mathrm{N}$ & Sound $\mathrm{P}, \mathrm{A}$ or $\mathrm{F}$ \\
\hline \multirow[t]{2}{*}{ Bombycidae } & B. mori $^{I}$ & $?$ & $\mathrm{~N}$ & - & 5 & $\mathrm{n} / \mathrm{a}$ & $\mathrm{n} / \mathrm{a}$ & $\mathrm{N}$ & - \\
\hline & O. angelica & 6 & $\mathrm{~N}$ & - & 5 & 7.45 & serrated & $\mathrm{N}$ & - \\
\hline \multirow[t]{18}{*}{ Saturniidae } & A. virginiensis & 12 & $\mathrm{~N}$ & - & 6 & 12.17 & smooth & $\mathrm{N}$ & - \\
\hline & C. lobesis & 2 & $\mathrm{Y}$ & chirp & 9 & 10.18 & serrated & $\mathrm{Y}$ & $\mathrm{P}, \mathrm{A}$ \\
\hline & D. rubicunda & 27 & $\mathrm{~N}$ & - & 5 & 14.38 & smooth & $\mathrm{Y}$ & - \\
\hline & E. imperialis & 11 & $\mathrm{~N}$ & - & 8 & 28.74 & serrated & $\mathrm{Y}$ & - \\
\hline & E. ormondei & 1 & $\mathrm{~N}$ & - & 10 & $\mathrm{n} / \mathrm{a}$ & $\mathrm{n} / \mathrm{a}$ & $\mathrm{Y}$ & - \\
\hline & S. santarosensis & 3 & $\mathrm{Y}$ & chirp & 6.5 & 34.04 & smooth & $\mathrm{Y}$ & $\mathrm{n} / \mathrm{a}$ \\
\hline & A. postalbida & 1 & $\mathrm{~N}$ & - & 10.5 & $\mathrm{n} / \mathrm{a}$ & $\mathrm{n} / \mathrm{a}$ & $\mathrm{Y}$ & - \\
\hline & A. io & 20 & $\mathrm{~N}$ & - & 6.5 & 23.81 & smooth & $\mathrm{Y}$ & - \\
\hline & A. phrynon & 1 & $\mathrm{~N}$ & - & 6.5 & $\mathrm{n} / \mathrm{a}$ & $\mathrm{n} / \mathrm{a}$ & $\mathrm{Y}$ & - \\
\hline & A. luna & 10 & $\mathrm{Y}$ & click & 6.5 & 26.04 & ridged and serrated & $\mathrm{Y}$ & $\mathrm{P}, \mathrm{A}$ \\
\hline & A. pernyi & 2 & $\mathrm{Y}$ & click & 9 & 27.05 & ridged and serrated & $\mathrm{Y}$ & $\mathrm{n} / \mathrm{a}$ \\
\hline & A. polyphemus & 150 & $\mathrm{Y}$ & click & 7.5 & 32.54 & ridged and serrated & $\mathrm{Y}$ & $\mathrm{P}$ \\
\hline & A. atlas & 8 & $\mathrm{~N}$ & - & 10 & 43.04 & smooth & $\mathrm{Y}$ & - \\
\hline & C. promethea & 34 & $\mathrm{Y}$ & click & 6 & 12.45 & smooth & $\mathrm{Y}$ & $\mathrm{F}$ \\
\hline & H. cecropia ${ }^{I}$ & $?$ & $\mathrm{~N}$ & - & 10 & $\mathrm{n} / \mathrm{a}$ & $\mathrm{n} / \mathrm{a}$ & $\mathrm{Y}$ & - \\
\hline & H. columbia ${ }^{I}$ & $?$ & $\mathrm{~N}$ & - & 8 & $\mathrm{n} / \mathrm{a}$ & $\mathrm{n} / \mathrm{a}$ & $\mathrm{n} / \mathrm{a}$ & - \\
\hline & R. fugax $^{2}$ & $\mathrm{n} / \mathrm{a}$ & $\mathrm{Y}$ & squeak & 6 & $\mathrm{n} / \mathrm{a}$ & $\mathrm{n} / \mathrm{a}$ & $\mathrm{n} / \mathrm{a}$ & $\mathrm{n} / \mathrm{a}$ \\
\hline & S. pyri & 52 & $\mathrm{Y}$ & chirp & 10 & 31.04 & serrated & $\mathrm{Y}$ & $\mathrm{P}, \mathrm{A}$ \\
\hline
\end{tabular}


Table 5.2 continued

\begin{tabular}{|c|c|c|c|c|c|c|c|c|c|}
\hline \multirow[b]{2}{*}{ Family } & \multirow[b]{2}{*}{ Species } & \multirow[b]{2}{*}{$\#$ tested } & \multicolumn{2}{|c|}{ Sound production } & \multicolumn{3}{|c|}{ Morphology } & \multicolumn{2}{|c|}{ Chemical defence } \\
\hline & & & $\mathrm{Y} / \mathrm{N}$ & Type & $\begin{array}{l}\text { Max body } \\
\text { length }(\mathrm{cm})^{3}\end{array}$ & $\begin{array}{c}\text { Head capsule } \\
\text { area }\left(\mathrm{mm}^{2}\right)\end{array}$ & Mandible type & $\mathrm{Y} / \mathrm{N}$ & Sound $\mathrm{P}, \mathrm{A}$ or $\mathrm{F}$ \\
\hline Sphingidae & A. ypsilon & 1 & $\mathrm{~N}$ & - & 9 & 28.88 & smooth & $\mathrm{N}$ & - \\
\hline & M. tiliae & 3 & $\mathrm{~N}$ & - & 6.5 & 14.82 & smooth & $\mathbf{N}$ & - \\
\hline & P. modesta ${ }^{I}$ & 8 & $\mathrm{~N}$ & - & 9 & $\mathrm{n} / \mathrm{a}$ & $\mathrm{n} / \mathrm{a}$ & $\mathrm{Y}$ & - \\
\hline & P. excaecatus & 14 & $\mathrm{~N}$ & - & 7.5 & 25.89 & smooth & $\bar{Y}$ & - \\
\hline & S. cerisyi & 10 & $\mathrm{~N}$ & - & $6^{*}$ & 21.32 & smooth & $\mathrm{Y}$ & - \\
\hline & S. jamaicensus & 5 & $\mathrm{~N}$ & - & 6 & 21.42 & smooth & $\bar{Y}$ & - \\
\hline & A. atropos & 5 & $\bar{Y}$ & click & 13 & $\mathrm{n} / \mathbf{a}$ & $\mathrm{n} / \mathrm{a}$ & $\mathrm{n} / \mathrm{a}$ & $\mathrm{n} / \mathrm{a}$ \\
\hline & C. undulosa & 20 & $\mathrm{~N}$ & - & 8.5 & 26.78 & ridged and serrated & $\mathrm{Y}$ & - \\
\hline & M. albiplaga & 1 & $\mathbf{N}$ & - & 12 & $\mathrm{n} / \mathrm{a}$ & $\mathrm{n} / \mathrm{a}$ & $\bar{Y}$ & - \\
\hline & S. drupiferarum & 16 & $\mathrm{~N}$ & - & $8.5^{*}$ & 31.98 & ridged and serrated & $\mathrm{Y}$ & - \\
\hline & S. kalmiae & 20 & $\mathrm{~N}$ & - & 8 & 22.29 & ridged and serrated & $\mathrm{N}$ & - \\
\hline & S. poecila & 18 & $\mathrm{~N}$ & - & 7.5 & 27.70 & ridged and serrated & $\mathrm{Y}$ & - \\
\hline & D. choerilus & 5 & $\mathrm{~N}$ & - & $5.5^{*}$ & 10.94 & serrated & $\mathrm{Y}$ & - \\
\hline & D. myron & 1 & $\mathrm{~N}$ & - & 5.5 & 12.07 & serrated & $\bar{Y}$ & - \\
\hline & D. inscriptum & 1 & $\mathrm{~N}$ & - & 5 & 11.49 & ridged and serrated & $\bar{Y}$ & - \\
\hline & E. satellitia & 2 & $\mathrm{Y}$ & click & 10 & 30.91 & ridged and serrated & $\mathrm{Y}$ & $?$ \\
\hline & H. euphorbiae ${ }^{1}$ & $\mathrm{n} / \mathrm{a}$ & $\mathrm{N}$ & - & 8 & $\mathrm{n} / \mathrm{a}$ & $\mathrm{n} / \mathrm{a}$ & $\mathrm{Y}$ & - \\
\hline
\end{tabular}


Table 5.2 continued

\begin{tabular}{|c|c|c|c|c|c|c|c|c|c|}
\hline \multirow[b]{2}{*}{ Family } & \multirow[b]{2}{*}{ Species } & \multirow[b]{2}{*}{ \# tested } & \multicolumn{2}{|c|}{ Sound production } & \multicolumn{3}{|c|}{ Morphology } & \multicolumn{2}{|c|}{ Chemical defence } \\
\hline & & & $\mathrm{Y} / \mathrm{N}$ & Type & $\begin{array}{c}\text { Max body } \\
\text { length }(\mathrm{cm})^{3}\end{array}$ & $\begin{array}{c}\text { Head capsule } \\
\text { area }\left(\mathrm{mm}^{2}\right)\end{array}$ & Mandible type & $\mathrm{Y} / \mathrm{N}$ & Sound $\mathrm{P}, \mathrm{A}$ or $\mathrm{F}$ \\
\hline \multirow[t]{4}{*}{ Sphingidae } & N. magna & 1 & $\mathrm{Y}$ & rasp & 6.5 & 27.59 & ridged and serrated & $\bar{Y}$ & $\mathrm{n} / \mathrm{a}$ \\
\hline & S. abbottii & 3 & $\mathrm{Y}$ & rasp & 7.5 & 20.97 & ridged and serrated & $\mathrm{N}$ & - \\
\hline & $X$. anubus & 1 & $\mathrm{~N}$ & - & 8.5 & $\mathrm{n} / \mathrm{a}$ & $\mathrm{n} / \mathrm{a}$ & $\mathrm{Y}$ & - \\
\hline & $X$. tyndarus & 1 & $\mathrm{~N}$ & - & 7 & $\mathrm{n} / \mathrm{a}$ & $\mathrm{n} / \mathrm{a}$ & $\mathrm{N}$ & - \\
\hline \multirow[t]{2}{*}{ Noctuidae } & A. sinuilinea & 1 & $\mathrm{~N}$ & - & $5.8^{\dagger}$ & $\mathrm{n} / \mathrm{a}$ & $\mathrm{n} / \mathrm{a}$ & $\mathrm{Y}$ & - \\
\hline & Unknown & 1 & $\mathrm{~N}$ & - & $4.5^{\dagger}$ & $\mathrm{n} / \mathrm{a}$ & $\mathrm{n} / \mathrm{a}$ & $Y$ & - \\
\hline \multirow[t]{3}{*}{ Notodontidae } & C. besckei & 1 & $\mathrm{~N}$ & - & $5^{\dagger}$ & $\mathrm{n} / \mathrm{a}$ & $\mathrm{n} / \mathrm{a}$ & $Y$ & - \\
\hline & L. fascis & 1 & $\mathrm{~N}$ & - & $8^{\dagger}$ & $\mathrm{n} / \mathrm{a}$ & $\mathrm{n} / \mathrm{a}$ & $\mathrm{N}$ & - \\
\hline & L. guatemalensis & 1 & $\mathrm{~N}$ & - & $4.7^{\dagger}$ & $\mathrm{n} / \mathrm{a}$ & $\mathrm{n} / \mathrm{a}$ & $\mathrm{N}$ & - \\
\hline Nymphalidae & Danaus sp. & 1 & $\mathrm{~N}$ & - & $3^{\dagger}$ & $\mathrm{n} / \mathrm{a}$ & $\mathrm{n} / \mathrm{a}$ & $\mathrm{Y}$ & - \\
\hline Hesperiidae & A. creteus crana & 1 & $\mathrm{~N}$ & - & $4.4^{\dagger}$ & $\mathrm{n} / \mathrm{a}$ & $\mathrm{n} / \mathrm{a}$ & $\mathrm{N}$ & - \\
\hline
\end{tabular}

No tapes available for analysis

${ }^{2}$ Information from literature only, type of sound described as per literature

${ }^{3}$ Max sizes reported for final instars in Sugi (1987), Wagner (2005), Janzen and Hallwachs (2009), Pittaway (1993, 2010) and Pittaway and Kitching (2010); ${ }^{*}$ indicates that sizes were estimated based on similarly sized adults; ${ }^{\dagger}$ indicates max sizes unavailable and size of tested specimen is reported 
Table 5.3 Spectral and temporal characteristics of sounds in those species for which information was available (mean \pm standard deviation, n's are listed in individual species sheets). More than one listed value indicates there is more than one type of sound for that species; details can be found in individual sheets.

\begin{tabular}{|c|c|c|c|c|c|c|c|c|}
\hline \multicolumn{2}{|c|}{ 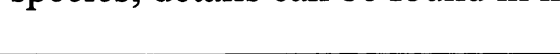 } & \multicolumn{3}{|c|}{ Spectral $^{1}$} & \multicolumn{4}{|c|}{ Temporal $^{\top}$} \\
\hline Mechanism & Species & $\begin{array}{l}\text { Dominant } \\
\text { frequency } \\
(\mathrm{kHz})\end{array}$ & Q3 & Q10 & Train duration (ms) & $\begin{array}{l}\text { \# pulses } \\
\text { in a train }\end{array}$ & $\begin{array}{l}\text { Pulse duration } \\
\text { (ms) }\end{array}$ & $\begin{array}{c}\# \\
\text { components } \\
\text { in a pulse }\end{array}$ \\
\hline \multirow[t]{7}{*}{ Mandible clicking } & A. atropos & $23 \pm 3$ & $3.51 \pm 1.28$ & $1.28 \pm 0.59$ & $3025 \pm 1514$ & $4-15$ & $14 \pm 3$ & $1-5$ \\
\hline & A. $\operatorname{luna}^{2}$ & $22 \pm 10$ & $\mathrm{n} / \mathrm{a}^{3}$ & $\mathrm{n} / \mathrm{a}$ & $3331 \pm 834$ & $1-11$ & $70 \pm 8$ & $1-4$ \\
\hline & A. polyphemus ${ }^{2}$ & $14 \pm 8$ & $8.67 \pm 2.21$ & $1.88 \pm 1.00$ & $7973 \pm 7664$ & $2-426$ & $25 \pm 17$ & $1-9$ \\
\hline & C. promethea & $34 \pm 7$ & $4.22 \pm 0.83$ & $1.19 \pm 0.49$ & $71 \pm 132$ & 1 & $71 \pm 132$ & $1-2$ \\
\hline & M. pellenia & $18 \pm 17$ & $2.62 \pm 1.47$ & $0.88 \pm 0.64$ & $2269 \pm 1339$ & $5-8$ & $3 \pm 8$ & $1-2$ \\
\hline & M. sexta & $28 \pm 10$ & $4.71 \pm 0.19$ & $1.36 \pm 0.41$ & $3490 \pm 4920$ & $2-38$ & $45 \pm 29$ & $1-4$ \\
\hline & E. satellitia & $\mathrm{n} / \mathrm{a}$ & $\mathrm{n} / \mathrm{a}$ & $\mathrm{n} / \mathrm{a}$ & $289 \pm 428$ & $1-4$ & $41 \pm 37$ & $1-4$ \\
\hline \multirow[t]{3}{*}{$\begin{array}{l}\text { Mandible } \\
\text { stridulation }\end{array}$} & C. lobesis & $32 \pm 11$ & $4.65 \pm 3.80$ & $0.64 \pm 0.14$ & $305 \pm 233$ & $1-3$ & $110 \pm 42$ & $6-32$ \\
\hline & S. santarosensis & $38 \pm 2$ & $4.38 \pm 2.22$ & $0.71 \pm 0.40$ & $194 \pm 246$ & $1-3$ & $57 \pm 15$ & $6-10$ \\
\hline & S. pyri ${ }^{4}$ & $\begin{array}{l}34 \pm 12 \\
22 \pm 15\end{array}$ & $\begin{array}{l}4.97 \pm 0.44 \\
3.07 \pm 1.17\end{array}$ & $\begin{array}{l}1.43 \pm 0.16 \\
1.10 \pm 0.40\end{array}$ & $1700 \pm 1120$ & $1-15$ & $\begin{array}{c}67 \pm 23 \\
9 \pm 10\end{array}$ & $\begin{array}{c}4-21 \\
1-3\end{array}$ \\
\hline \multirow[t]{3}{*}{$\begin{array}{l}\text { Air expulsion - } \\
\text { buccal }^{5}\end{array}$} & N. magna & $25 \pm 13$ & $6.20 \pm 7.09$ & $1.33 \pm 1.31$ & $3860 \pm 1392$ & $8-15$ & $50 \pm 11$ & $6-21$ \\
\hline & P. drucei & $43 \pm 11$ & $2.85 \pm 2.30$ & $0.69 \pm 0.24$ & $178 \pm 24$ & $1-2$ & $102 \pm 86$ & $2-43$ \\
\hline & S. abbottii & $\begin{array}{l}33 \pm 15 \\
25 \pm 14^{6}\end{array}$ & $\begin{array}{l}9.49 \pm 3.17 \\
9.16 \pm 4.48\end{array}$ & $\begin{array}{l}0.95 \pm 0.23 \\
3.17 \pm 2.14\end{array}$ & $6906 \pm 3732$ & $7-36$ & $\begin{array}{c}82 \pm 24 \\
0.74 \pm 0.74\end{array}$ & $\begin{array}{c}1-30 \\
1-3\end{array}$ \\
\hline $\begin{array}{l}\text { Air expulsion - } \\
\text { spiracles }\end{array}$ & A. juglandis ${ }^{4}$ & $\begin{array}{l}22 \pm 8 \\
15 \pm 2 \\
10 \pm 4\end{array}$ & $\begin{array}{c}40.67 \pm 42.38 \\
7.39 \pm 5.22 \\
19.00 \pm 5.67\end{array}$ & $\begin{array}{c}13.81 \pm 7.86 \\
1.02 \pm 0.27 \\
9.90 \pm 3.79\end{array}$ & $2626 \pm 2339$ & $1-8$ & $440 \pm 272$ & - \\
\hline
\end{tabular}

\footnotetext{
These terms are explained and illustrated in Figure 5.1

${ }^{2}$ Some information on these species was obtained from Brown et al. (2007)

${ }^{3} \mathrm{n} / \mathrm{a}$ indicates that information is not available due to recordings being made at a low sampling rate or were unavailable

${ }^{4}$ Sounds were divided into different 'types' based on spectral analysis

${ }^{5}$ This is the assumed mechanism and remains unverified

${ }^{6}$ These sounds are considered to be clicks
} 
Taxonomic distribution of airborne sound production in Lepidoptera caterpillars

The order Lepidoptera contains 46 superfamilies according to Kristensen and Skalski (1998). The taxon Macrolepidoptera is a monopheletic group that consists of medium to large sized species with broad wings and exophagous larvae in 11 superfamilies (Fig 5.70, Kristensen and Skalski 1998). Bombycoidea, Papilionoidea and Hesperoidea possess, on average, the largest sized species, and therefore include some of the most striking and aesthetically pleasing adult specimens that have been reared from egg and caterpillar by both professional and amateur collectors and enthusiasts. Of course, these groups also include some species that are smaller, for example, the adults of D. rubicunda and $O$. angelica have wingspans of 40 and $45 \mathrm{~mm}$, compared to the wingspans of $C$. undulosa and A. polyphemus at 89 and $100 \mathrm{~mm}$ (Troubridge and Lafontaine 2004). In addition, taxa that are considered to typically have small to medium sized moths may also contain species that are larger than average. For example the family Noctuidae contains species that have wingspans that range from $13 \mathrm{~mm}$ to $300 \mathrm{~mm}$ (Wagner 2005). Given the observation that sound production has been previously overlooked in species such as Manduca sexta, which is one of the most widely studied insects, it is possible that sound production in larger caterpillars may be widely distributed throughout the Lepidoptera, but have just gone unnoticed. Therefore, I took every opportunity to test any species that was large, since large species are capable of producing sounds loud enough to be heard by a vertebrate predator (discussed in Part C). As seen from my results (Figs. 5.59-5.69), none of these species produced audible sounds, although one species may have produced vibrations (Fig 5.65). Although, naturally, more species need to be tested throughout the Lepidoptera, I propose that 


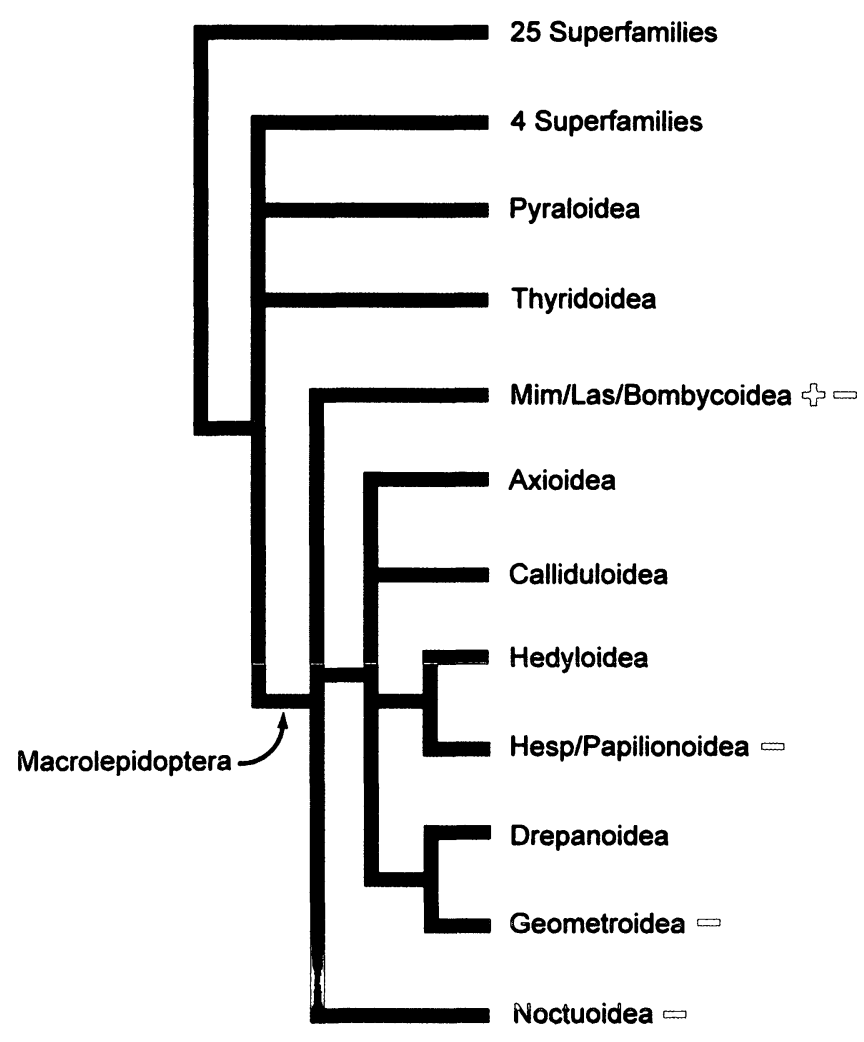

Figure 5.70 A phylogeny of the superfamilies of the Lepidoptera, modified from Kristensen and Skalski (1998), showing the presence $(+)$ or absence $(-)$ of sound production in tested groups. 
sound production is probably most common, if not restricted to the members of the Bombycoidea for the following reasons: (a) all of the previous accounts of sound production mentioned by naturalists have been for the Bombycoidea, and (b) the other groups with large caterpillars (i.e. Papilionoidea, Hesperoidea) have been widely studied by scientists and naturalists alike (e.g. rearing for leisure, as well as studies on caterpillar defences), and sound production in these caterpillars should have been noted at least once by now. Assuming that sound production is most common in Bombycoidea caterpillars, why might this be? This question will be addressed in Part $\mathrm{C}$, and the remainder of this section will focus on the taxonomic distribution of sound production within the Bombycoidea.

As mentioned previously, the Bombycoidea superfamily contains ten families, the most speciose of which are the Saturniidae and the Sphingidae (Regier et al. 2008a,b, Kawahara et al. 2009). Sound production was observed in 2 of the 3 tested Saturniidae subfamilies, all of the tested Sphingidae subfamilies, and neither of the two tested Bombycidae subfamilies (Fig 5.71). Within the subfamilies that contain sound producing species, at least four different mechanisms were noted; mandible clicking, mandible stridulation, the expulsion of air through the spiracles and, possibly, the expulsion of air through the buccal cavity over a membrane (Fig 5.72). The production of sound by the movement of air out of the spiracles is the least common mechanism noted to date, and the only mechanism present in the subfamily Smerinthinae. It has been confirmed in $A$. juglandis and suggested, but remains unconfirmed, for Phyllosphingia dissimilis. Interestingly, these two species are sister species and the next most closely related species (M. tiliae, Kawahara et al. 2009) did not produce sounds, suggesting this mechanism may 


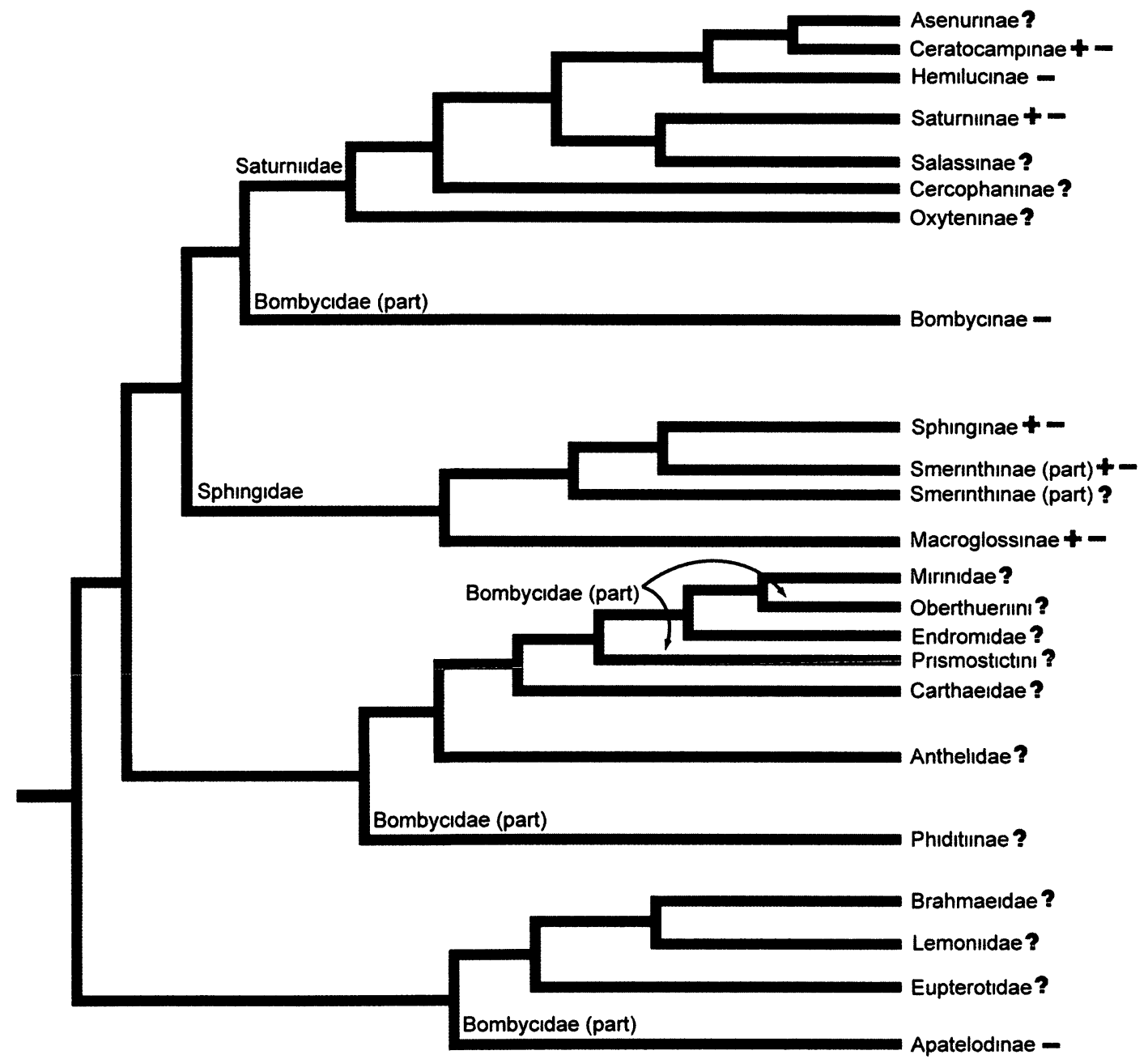

Figure 5.71 Phylogeny of the Bombycoidea adapted from Regier et al. (2008a), showing the presence $(+)$ or absence $(-)$ of sound production in each examined taxon. Taxa in which no species have been tested to date are denoted by '?' 


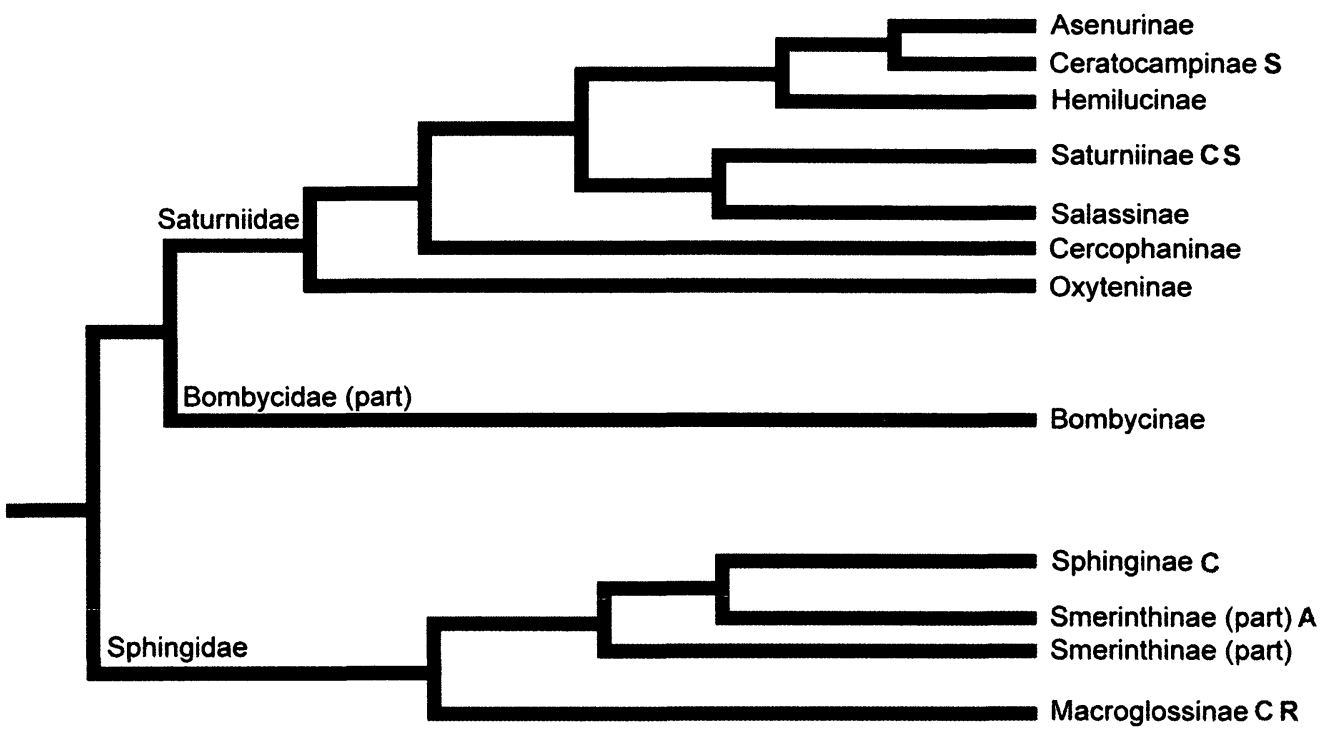

Figure 5.72 Phylogeny of the subfamilies of Saturniidae and Sphingidae demonstrating the distribution of different sound producing mechanisms; $\mathrm{C}$ - mandible clicking, $\mathrm{S}$ - mandible stridulation (chirping), A - air expulsion through spiracles, $\mathrm{R}$ - air expulsion through buccal cavity (rasp). Phylogeny modified from Regier et al. (2008a). 
not be observed elsewhere in the group. Mandible clicking was the most common mechanism observed. It was present in eight species from three diverse subfamilies indicating it may have evolved independently at least twice. Mandible stridulation was distinct from mandible clicking by the number of components within each pulse of sound (higher for stridulation). It was noted three times, all within the Saturniidae: once in a subfamily that also had species that clicked (Saturniinae) and twice in a subfamily that did not (Ceratocampinae). The movement of air through the buccal cavity (suspected) to produce sound was noted only in the subfamily Macroglossinae. The three species that used this mechanism all belong to the clade "Diplophontina sensu lato" (Kawahara et al. 2009). For each mechanism, it will be interesting to examine the taxonomic origins by testing additional species in the various groups.

Tentatively mapping sound production onto a previously published phylogeny demonstrates the distribution of the characters but does not accurately document its evolutionary history. Different mechanisms may have evolved independently a number of times or one mechanism may have evolved into another. Alternatively all caterpillars may have produced sound ancestrally and the ability has been lost several times. Future studies should focus on increasing the dataset and use phylogenetic analyses to map behavioural characters in order to determine the most likely evolutionary scenario. At present, my data offers the following general conclusions: sound production is, to date, restricted to the Bombycoidea, and throughout the group, sound production is widespread with at least four different mechansisms (although mandible clicking may be considered mandible stridulation). Hypotheses on the evolutionary origins of signals from non 
signals, the function of signals, and proposed selection pressures that result in sound production will be discussed in the following section.

\subsection{PART B: Evolutionary Origins of Communication signals}

\subsubsection{Introduction}

In this thesis I have provided evidence that caterpillars in the superfamily Bombycoidea can use at least three different body regions to produce sound: the mandibles, spiracles, and some internal mechanism in the foregut or buccal region. The evolution of 3 different sound producing mechanisms within one insect superfamily is impressive, but even more impressive due to the fact that caterpillars are soft-bodied, and do not posses the hard exoskeleton that enables a variety of options in other insects. The introduction of these various sound production mechanisms begs the question: How do these communication signals arise? In this section I will discuss the possible evolutionary origins of these signals.

The evolution of signals has intrigued scientists for a long while, and the subject was first considered in detail by the early ethologists who used comparisons between behaviours within or between species to develop hypotheses about the origin of signals (e.g. Tinbergen 1952, Crane 1966, Randall 2001). It is hypothesized that communication between a sender and a receiver begins with the association of a cue and a condition (Bradbury and Vehrencamp 1998). Inadvertent cues generated by the sender as a byproduct of some other behaviour may become ritualized into a signal if they are recognised by the receiver and the fitness of the sender is increased. The process of 
ritualization is thought to enhance the efficacy of these cues through one or more of the following modifications to the cue: a. simplification or reduction in the number of components; $b$. exaggeration of remaining components; $c$. repetition of the signal and d. increased stereotypy (Bradbury and Vehrencamp 1998). One or more of these modifications make it easier to perceive the signal by increasing their reliability, distortion resistance and information content (Bradbury and Vehrencamp 1998). For example, in insects, the majority of sounds are generated by some type of external limb movement which could previously have been employed for some other function, like flying, walking, grooming, feeding, respiratory stress or struggling (Haskell 1974). Hypothetically, an orthopteran employing femoral-abdominal stridulation to produce sound would raise its leg and bring it back down while contacting the body. This motion presumably involves fewer steps than when using the leg for walking (e.g. towards a mate) and the range of motion covered by the leg is likely larger. When observing the two behaviours separately but looking at the same amount of elapsed time, the motion used to produce sounds is probably repeated more than the motion used for walking (i.e. repetition of the signal) and there is likely more variation in the movements associated with walking than with signalling (i.e. increased stereotypy).

Many hypotheses have been proposed to explain the origin of signals, but experimental evidence is lacking, since it requires studying closely related species with a variation of signalling behaviour within a phylogenetic framework, as recently shown in Scott et al. (2010a). Typically however, hypotheses are inferred, or tested from comparisons of behaviours between or within species. My current data set on sound production in caterpillars lacks robust representation from key groups, and therefore it is 
not possible to formally test hypotheses on the origins of signals. The purpose of this section is to examine the variation in the documented behaviours in different species, and develop hypotheses on the evolutionary origins of the sound production mechanisms from non-signalling behaviours for future testing.

\subsubsection{Methods}

Variation of acoustic signals and/or cues associated with other behaviours was noted in the species surveyed in the previous section (Part A). Based on these signal variations I propose possible hypotheses to explain the evolutionary origins of the three different signal types: mandible signals (clicking or stridulation), forced air through spiracles, and the expulsion of air through the buccal cavity. Since the greatest variation in signal complexity was noted for mandibular sounds, I also attempted to characterize the possible pathway to ritualization. Quantitative measures for the ritualization of mandibular sounds (inadvertent cues to clicking and then stridulation) were assessed in C. promethea, A. luna, A. polyphemus and S. pyri. Temporal and relative amplitude characteristics were measured from a maximum of five trains from up to five individuals using Raven and AviSoft SASlab Pro as follows:

Exaggeration of existing components - The relative amplitude of sound recordings with the same baseline were compared.

Repetition of the signal - The number of components produced within one movement (i.e. the production of one pulse) were counted and compared. 
Stereotypy - The interval between components produced within one movement was determined and the variance between these measurements was compared (measured as standard deviation of the mean).

\subsubsection{Results and Discussion}

Proposed origins of mandible signals

Biting, regurgitation and two mechanisms of sound production (clicking and stridulation) were the behaviours exhibited by Bombycoidea caterpillars that involved the use of the mandibles. Of these behaviours, any incidental sounds produced during biting or regurgitation may act as unintentional cues to a listening predator, while sounds produced by clicking and stridulation are considered intentional signals. The action of the mandibles during sound production appears similar to their action during feeding- the anterior edge of one mandible slides against the inner face of the opposing mandible (Bernays and Janzen 1988). The same motor pattern may also occur during biting or regurgitation (as material moves out through the mouth) producing incidental sounds. These cues may have formed an association perceived by a predator reducing the likelihood of consumption and increasing the benefits of a signal. Sounds would eventually begin to precede biting or regurgitation, or regurgitation may eventually be lost, or replaced with another chemical defence. Observations within the subfamily Saturniinae may provide an interesting case in which to observe the process of ritualization from cue to signal. Different levels and types of sound production were observed in four species; incidental clicks in C. promethea, clicking in A. luna, increased 
clicking in A. polyphemus and stridulation in S. pyri (Fig 5.73). Using the criteria outlined in the introduction to this section I could preliminarily test the hypothesis that incidental clicks evolved through the process of ritualization to clicking or stridulation. Three criteria were met; exaggeration of components, repetition of the signal and increased stereotypy (or decreased variance). The exaggeration of components can be seen in the increase in relative amplitude of the signals, which is easy to visualize when given a standardized baseline. The repetition of components within the production of a single sound increased from 1-2 in C. promethea, to 1-4 and 1-9 in A. luna and A. polyphemus respectively, and finally 1-21 in $S$. pyri sounds. Finally, the variance in the interval between components within a sound decreased from 0.011 in C. promethea to 0.002 in $S$. pyri. Though this preliminary evidence suggests that there is some merit to this hypothesis, a more thorough examination is required before any conclusions can be made, including whether incidental clicks were ritualized into both clicking and stridulation or if incidental clicks were ritualized into clicks and further ritualized into stridulation.

\section{Proposed origins of spiracular sounds}

Defensive behaviours involving bodily contractions included twitching, thrashing and forcing air through the spiracles to produce sound. The two former behaviours only produced incidental sounds by hitting the body against the substrate, while the latter produced clear signals. I propose that the longitudinal contractions used to force air through the spiracles in the walnut sphinx caterpillar were assimilated from telescoping movements used to increase ventilation (Fig 5.74). When a caterpillar is attacked it may 

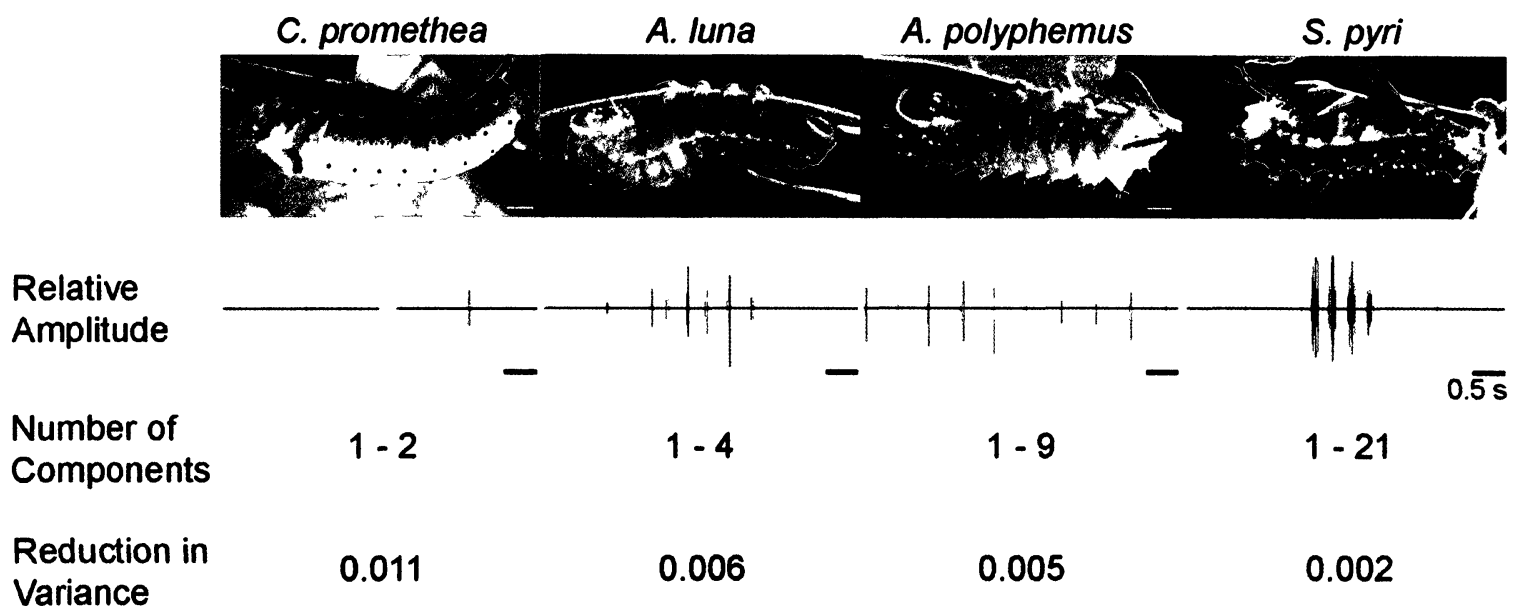

Figure 5.73 A hypothesis for the ritualization of incidental clicks to clicking and stridulation in four species from the same subfamily. The relative amplitude of the signals increases with complexity, as does the repetition (number of components in a sound), while the variance within one movement (interval between components within a sound) decreases. 
a

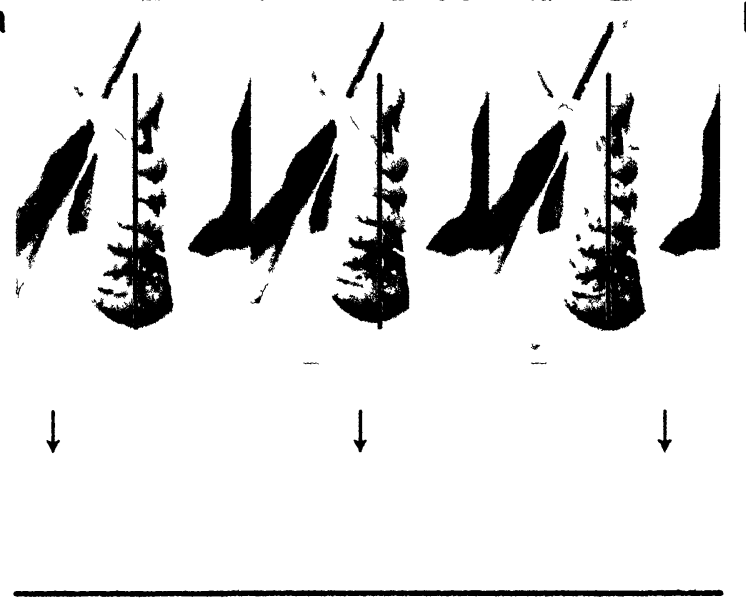

$0 \overline{1 s}$

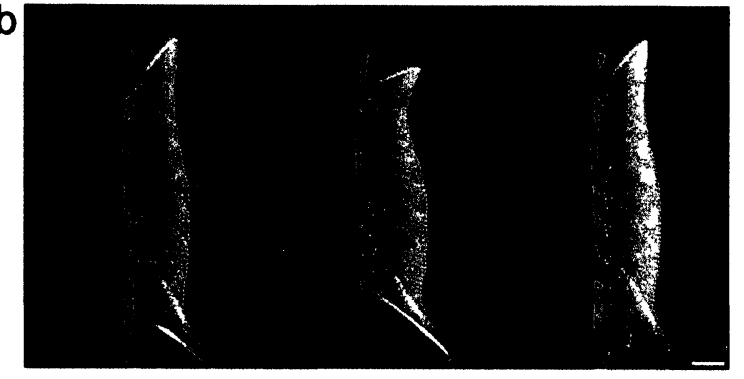

$\downarrow \quad \downarrow \downarrow$

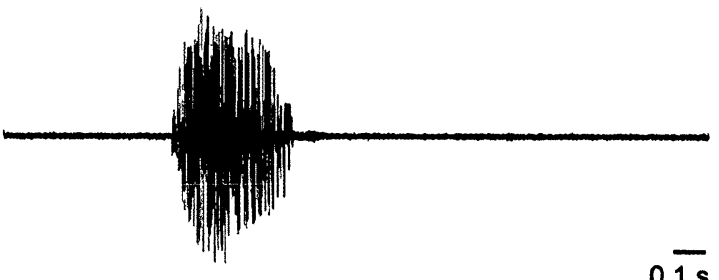

Figure 5.74 Proposed evolution of air expulsion through the spiracles from twitching. (a) Twitching in a $C$. undulosa caterpillar accompanied by silence, the vertical black line is shown to aid in discriminating the movement. Arrows indicate the occurrence of the video stills. (b) Sound production in $A$. juglandls accompanied by a longitudinal contraction. Arrows indicate the occurrence of the video stills. 
ventilate its system in preparation for the increased activity involved in defensive behaviours. My observations of twitching lend some evidence to this theory as 9 species (100\% that twitched) were seen to twitch prior to thrashing or regurgitation (e.g. A. luna, P. drucei). Bodily contractions to increase ventilation are seen in other insects, especially those that are very active (Miller 1974).

\section{Proposed origins of rasping sounds}

Regurgitation and the rasping mechanism are behaviours that involved the mouth and foregut area of the caterpillar. Noisy regurgitation would be a cue, while rasps would be considered true signals. The mechanism of rasping has not been confirmed, but I suggest it is created by the movement of air over a membrane through the foregut or buccal cavity. As mentioned in Chapter 4, during the first part of the cry of adult $A$. atropos air is sucked in through the proboscis and causes the epipharynx to vibrate, producing sound. The similarity in temporal structure between these sounds and those produced by the caterpillars in this study (Fig. 5.75) lend some support to the possibility, as does the observation that sounds occur as the mandibles are separated and open. I suggest that this mechanism may have evolved from the noisy regurgitation observed in other species (e.g. E. oromondei) (Fig 5.76). Incidental sounds in these species may be caused by a small amount of ingested air escaping with the regurgitant, which may be perceived by a predator and reinforce an association between the sound and the defence, eventually occurring before the regurgitation or without it completely (e.g. S. abbottii). 
Future studies to further test all the proposed hypotheses should involve increased sampling from closely related groups, phylogenetic analysis and quantitative examination of the process of ritualization. 
a

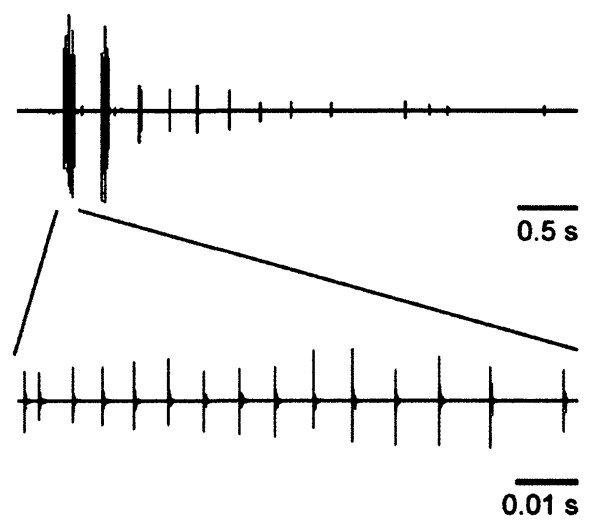

b

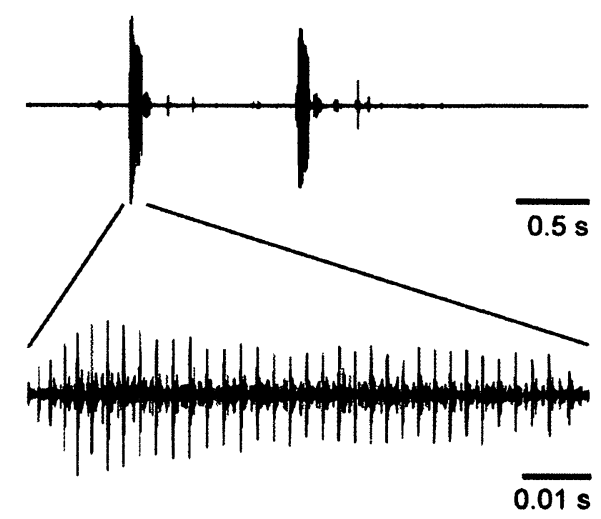

Figure 5.75 Comparison of sounds made by $S$. abbottii to those made by $A$. atropos. (a) Oscillogram and expanded time scale of sounds produced by larvae of $S$. abbottii. (b) Oscillogram and expanded time scale of sound produced by adult $A$. atropos. 
a
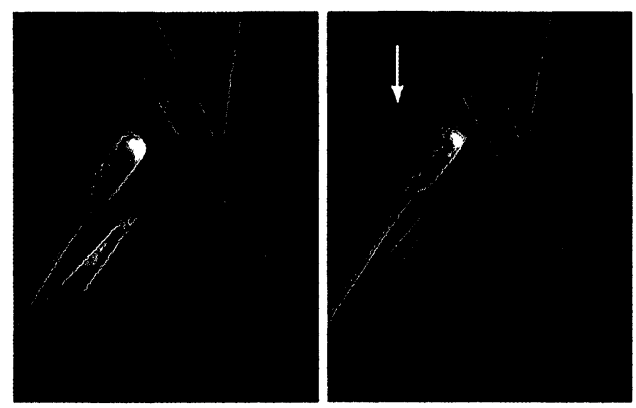

$\downarrow \downarrow$

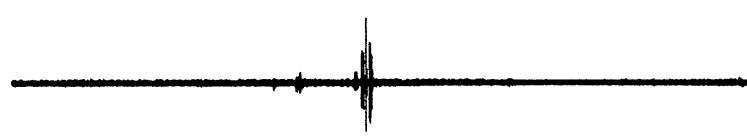

$0 . \overline{05} \mathrm{~s}$ b
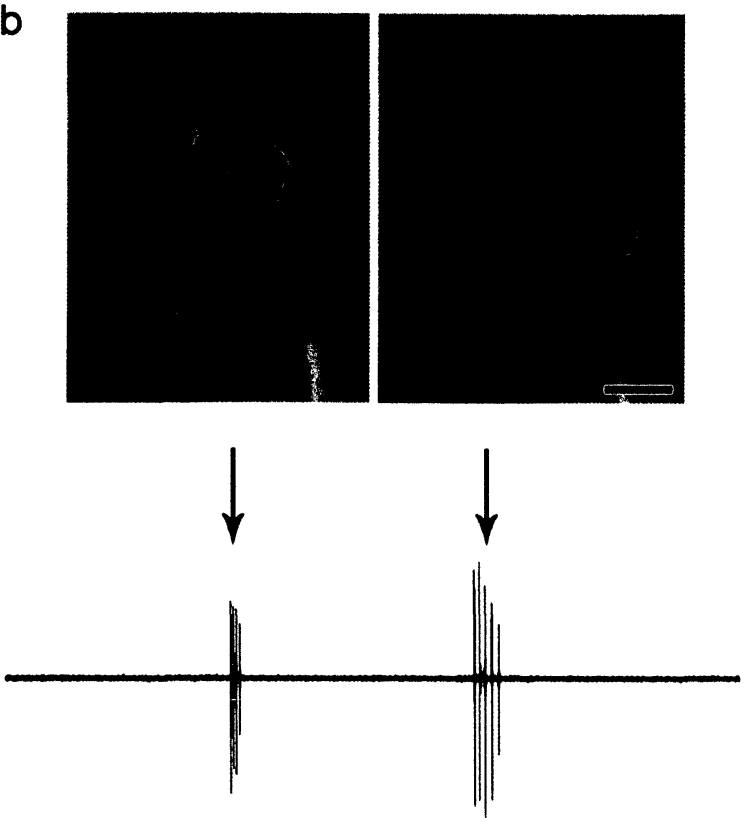

$0 . \overline{05 s}$

Figure 5.76 Hypotheses for the evolution of the rasping mechanism from noisy regurgitation. (a) Noisy regurgitation in an $E$. ormondei caterpillar. Black arrows indicate the occurrence of the video still frames, the white arrow is pointing to a bubble of regurgitation. (b) Sound production in a $S$. abbottii caterpillar. Arrows indicate the occurrence of the video still frames, note that the mandibles are open when sounds are produced. 


\subsection{PART C: Why do Some Caterpillars use Defensive Sounds?}

\subsubsection{Introduction}

In this thesis, I have introduced several novel instances of sound production in Bombycoidea caterpillars, demonstrating that this phenomenon appears to be relatively common in this superfamily. Fifteen out of 42 Bombycoidea species tested produced sounds, comprising about a third of all species tested. It is without a doubt that sound production will be described in more species in the future. These sounds are not only diverse in their mechanisms of production, but, from what I can conclude from the data gathered on the four species that have been studied in some detail, namely, $A$.

polyphemus (Brown et al. 2007), M. sexta, S. pyri, and A. jugulandis (this thesis), their functions may also be variable. In Chapters 2 and 3, I concluded that sound production functions as a warning of an impending chemical defence, regurgitation or scolus secretions in $M$. sexta and S. pyri respectively. In these cases, as in the previously reported case for A. polyphemus (Brown et al. 2007), the sounds produced by the caterpillars either preceded or accompanied their respective defences. In Chapter 4 this association between sound production and chemical defence was not observed in the whistling walnut sphinx (A. jugulandis) and I concluded that the sounds were an example of a startle defence, or possibly a general signal of unprofitability. Function, like mechanism, is probably diverse among species, which, as we have seen in Part A, also had various combinations of defences during the attack experiments. In this final section I will incorporate information obtained from the survey to address questions regarding the function of acoustic defences in caterpillars. I will then examine my data and attempt to 
develop hypotheses to explain the variation in sound production throughout the Bombycoidea.

\subsubsection{Results and Discussion}

\section{What is the function of airborne sound production in caterpillars?}

In Chapter 1 I made the assumption that sounds produced by these caterpillars were defensive in nature, and introduced various hypotheses that might explain the specific function of acoustic defence signals in insects. The data collected during the course of my thesis support the hypothesis that sounds are defensive because all sounds were associated with attack and other defensive behaviours (Table 5.4, 5.5) and were not observed during interactions between conspecifics or during standard activities such as feeding or walking. In this section I will review the various hypotheses proposed earlier on the function of acoustic defence sounds in light of the data collected. These included warning (aposematism), startle, jamming, recruitment, mimicry, enhanced learning and unprofitability. It should be kept in mind however, that sounds produced by any particular caterpillar could, and probably do, affect different predators in different ways. What serves as a warning signal in one species may simply startle a second, or tell a third that this prey animal would be too time consuming, or have no effect at all on a fourth. For example, the startling eye-spots of some hawkmoths and peacock butterflies were more successful against blue tits than great tits (Vallin et al. 2007) and the chemical effluent of a harvestman was extremely effective against ants while it was only mildly irritating to wolf spiders (Eisner et al. 2004). 
Table 5.4 Behaviours observed in 42 species of Bombycoidea caterpillars. 'Y's indicate that the behaviour was present. The total number of species exhibiting that behaviour at least once, and the percentage of species that exhibited that behaviour are indicated at the bottom of the table.

\begin{tabular}{|c|c|c|c|c|c|c|c|c|c|c|c|c|}
\hline Species & Sound production & Regurgitation & Chemical defence & Thrashing & Major thrashing & Directed thrashing & Biting & Curling-in & Curlıng-up & Twitching & Walk away & Dropping \\
\hline$O$ angelica & & & & & & $\mathrm{y}$ & & $y$ & & & & $y$ \\
\hline$A$ virgimensis & & & & & & & & & & & & \\
\hline$C$ lobesis & $y$ & $y$ & & & & $\mathbf{y}$ & & & & & $\mathbf{y}$ & \\
\hline D rubicunda & & $\mathbf{y}$ & & & & $\mathbf{y}$ & & & & & & \\
\hline E imperialis & & $y$ & & $y$ & & $y$ & & $y$ & & & & \\
\hline E ormonder & & $\mathbf{y}$ & & $\mathbf{y}$ & & $\mathbf{y}$ & & & & $y$ & & \\
\hline$S$ santarosensis & $\mathbf{y}$ & $\bar{y}$ & & $y$ & & $\bar{y}$ & & & & $\mathbf{y}$ & & \\
\hline A postalbida & & & $y$ & & & $\mathbf{y}$ & & $\mathbf{y}$ & & & & \\
\hline$A 10$ & & & $y$ & & & $\mathbf{y}$ & & $\mathbf{y}$ & $\mathbf{y}$ & & & \\
\hline A phrynon & & & $y$ & & & $y$ & & & & & $\mathbf{y}$ & \\
\hline$A$ luna & $y$ & $y$ & & $y$ & & $y$ & & $y$ & & $y$ & & \\
\hline A pernyl & $y$ & $y$ & & $\bar{y}$ & & $\mathbf{y}$ & & $\mathbf{y}$ & & & & \\
\hline A polyphemus & $y$ & $y$ & & $\mathbf{y}$ & & $\mathbf{y}$ & & $\mathbf{y}$ & & & & \\
\hline$A$ atlas & & & $\bar{y}$ & & & $\mathbf{y}$ & & $\mathbf{y}$ & & & & \\
\hline C promethea & $y$ & $\mathbf{y}$ & & & & $\mathbf{y}$ & & $y$ & & & & \\
\hline$S$ pyr & $\mathrm{y}$ & & $\mathrm{y}$ & & & $\mathbf{y}$ & & $\mathbf{y}$ & & & & \\
\hline A ypsilon & & & & & $y$ & $\bar{y}$ & & & & & & \\
\hline$A$ Juglandis & $\mathbf{y}$ & $y$ & & & & $y$ & $y$ & & & & & \\
\hline$M$ thlae & & & & & $\mathbf{y}$ & $y$ & & & & & & $\mathbf{y}$ \\
\hline$P$ excaecatus & & $y$ & & & & $\mathrm{y}$ & & $\mathbf{y}$ & & & & \\
\hline$P$ myops & & $y$ & & & & & $\mathbf{y}$ & $y$ & & & & \\
\hline$S$ cerisyı & & $\mathbf{y}$ & & & & $y$ & & $\mathbf{y}$ & & & & \\
\hline$S$ jamaicensus & & $y$ & & & & $y$ & & $\mathbf{y}$ & & & & \\
\hline$A$ atropos & $y$ & & & & & & $\mathbf{y}$ & & & & & \\
\hline$C$ undulosa & & $\mathrm{y}$ & & $y$ & & $y$ & & $\mathrm{y}$ & & $y$ & & \\
\hline$M$ albiplaga & & $\mathrm{y}$ & & & $\mathbf{y}$ & $\mathbf{y}$ & & & & & & \\
\hline$M$ floristan & & & & & $\mathbf{y}$ & & & & & & & \\
\hline$M$ pellenia & $\mathbf{y}$ & $\mathbf{y}$ & & & $\mathbf{y}$ & $\mathbf{y}$ & $\mathbf{y}$ & & & & & \\
\hline$M$ sexta & $\mathbf{y}$ & $\mathbf{y}$ & & $y$ & & $\mathbf{y}$ & $y$ & $\mathbf{y}$ & & & & \\
\hline$S$ chersis & & & & $\mathrm{y}$ & & $\mathbf{y}$ & $\mathrm{y}$ & $\mathrm{y}$ & & & & \\
\hline$S$ drupiferarum & & $\mathrm{y}$ & & $\mathrm{y}$ & & $\mathrm{y}$ & $\mathrm{y}$ & $\mathrm{y}$ & & $\mathbf{y}$ & & \\
\hline$S$ kalmiae & & & & $y$ & & $y$ & & $y$ & & & & \\
\hline S poecila & & $y$ & & & & $y$ & $\mathbf{y}$ & $\mathbf{y}$ & & & & \\
\hline$D$ choerilus & & $y$ & & $\mathbf{y}$ & & $\mathbf{y}$ & & $\mathbf{y}$ & & $y$ & & \\
\hline D myron & & $\mathrm{y}$ & & $\mathrm{y}$ & & $\mathbf{y}$ & & $y$ & & & & \\
\hline$D$ inscriptum & & $y$ & & $y$ & & $\mathrm{y}$ & $\mathbf{y}$ & $y$ & & & & \\
\hline$E$ satellitia & $\mathbf{y}$ & $\mathbf{y}$ & & $\mathbf{y}$ & & $\mathbf{y}$ & & $\mathbf{y}$ & & & $\mathbf{y}$ & \\
\hline$N$ magna & $y$ & $y$ & & & $\mathbf{y}$ & $y$ & & & & & & \\
\hline$P$ drucel & $y$ & $y$ & & $\mathbf{y}$ & & $\mathrm{y}$ & & & & $\mathbf{y}$ & & \\
\hline$S$ abbottu & $y$ & & & $\mathbf{y}$ & & $y$ & $\mathbf{y}$ & & $\mathbf{y}$ & & & $y$ \\
\hline$X$ amubus & & $\mathbf{y}$ & & & & $y$ & & $\mathbf{y}$ & & $\mathbf{y}$ & $\mathbf{y}$ & \\
\hline$X$ tyndarus & & & & $y$ & & $\mathrm{y}$ & & $\mathrm{y}$ & & $y$ & & $y$ \\
\hline Total \# of species & 15 & 27 & 5 & 18 & 6 & 38 & 10 & 26 & $\mathbf{2}$ & 9 & 4 & 4 \\
\hline Percentage of species & 35.7 & 64.3 & 11.9 & 42.9 & 14.3 & 90.5 & 23.8 & 61.9 & 4.8 & 21.4 & 9.5 & 9.5 \\
\hline
\end{tabular}


Table 5.5 Combinations of defensive behaviours observed in 42 species of Bombycoidea caterpillars. Values represent the number of times the two listed behaviours occurred together in a particular species. The total number of species exhibiting a behaviour is indicated in the top row.

\begin{tabular}{|c|c|c|c|c|c|c|c|c|c|c|c|c|}
\hline & $\begin{array}{c}\text { Sound } \\
\text { production }\end{array}$ & Regurgitation & $\begin{array}{l}\text { Chemical } \\
\text { defence }\end{array}$ & Thrashing & $\begin{array}{c}\text { Major } \\
\text { thrashing }\end{array}$ & $\begin{array}{l}\text { Directed } \\
\text { thrashing }\end{array}$ & Biting & $\begin{array}{l}\text { Curling- } \\
\text { in }\end{array}$ & $\begin{array}{l}\text { Curling- } \\
\text { up }\end{array}$ & Twitching & $\begin{array}{l}\text { Walk } \\
\text { away }\end{array}$ & Dropping \\
\hline \multicolumn{13}{|l|}{$\begin{array}{l}\text { Sound } \\
\text { production }\end{array}$} \\
\hline $\begin{array}{l}\text { Chemical } \\
\text { defence }\end{array}$ & 1 & 0 & & & & & & & & & & \\
\hline Thrashing & 8 & 14 & 0 & & & & & & & & & \\
\hline $\begin{array}{l}\text { Directed } \\
\text { thrashing }\end{array}$ & 14 & 26 & 5 & 18 & 5 & & & & & & & \\
\hline Biting & 5 & 7 & 0 & 5 & 1 & 8 & & & & & & \\
\hline Curling-in & 7 & 18 & 4 & 14 & 0 & 25 & 5 & & & & & \\
\hline Curling-up & 1 & 0 & 1 & 1 & 0 & 2 & 1 & 1 & & & & \\
\hline Twitching & 3 & 7 & 0 & 8 & 0 & 9 & 0 & 6 & 0 & & & \\
\hline Walk away & 2 & 3 & 0 & 1 & 0 & 4 & 0 & 2 & 0 & 1 & & \\
\hline
\end{tabular}


Before further discussing the function of acoustic signals in caterpillars it is necessary to consider the predators of these insects. It is not easy to obtain specific information on the exact predators of any particular caterpillar species, since this necessitates direct observations of predator-prey interactions. In some cases I have been able to obtain specific information on these interactions from the literature, which I have listed in the survey sheets. Even within a species, the types and number of predators will vary depending on the geographic region. Therefore, the information I am able to present regarding predators that are likely to attack Bombycoidea caterpillars is from general reviews on the families. Insectivorous birds are considered a major predator and other vertebrates such as bats, shrews and mice have been reported to eat caterpillars (Pittaway 1993, Tuskes et al. 1996, Tuttle 2007).

The characteristics of the sounds produced by Bombycoidea caterpillars share features that are typically associated with other defensive sounds so they can be perceived, and recognised, by the differing hearing abilities of their various predators. Though sound level was not measured for every species that produced sound, signals were clearly audible at close range and should be easy for a predator to perceive. At a distance of $10 \mathrm{~cm}$, sounds ranged from $74.2-93.9 \mathrm{~dB}$ SPL (for the three species where it was measured), well above the typical noise floor in both temperate and tropical forests (30 and $50 \mathrm{~dB}$ SPL respectively, Goerlitz et al. 2008). In a quiet room sounds could be heard even at distances of $2 \mathrm{~m}$ with the unaided human ear, however, in noisier environments the sounds became indistinguishable from the background at about $1 \mathrm{~m}$. If the sounds continued for a while after an initial attack had been abandoned (can last for $>1 \mathrm{~min}$ in some species, see Table 5.3), the quietness of the sounds at a distance would 
make it unlikely that another predator could orient towards them and discover the caterpillar. The temporal characteristics exhibited by the sounds of these caterpillars can also make it difficult for a predator to localize them. The majority of the sounds had a high amount of variation and pulses typically lasted less than $100 \mathrm{~ms}(12 / 14$ species, Table 5.3). Short sounds can make it more difficult for a predator to find a prey item if they use arrival time information to pinpoint its location (Bradbury and Vehrencamp 1998). The temporal features of the caterpillars' signals were consistent with the defence sounds recorded by Masters (1980) which were also less than $100 \mathrm{~ms}$ in duration and lacked a clear structure. In social scenarios the temporal structure of a song is very important for species recognition (Haskell 1961, Bailey 2003) whereas the lack of structure in defensive contexts may be important in producing a broad frequency spectrum (Haskell 1961). At least one pulse type from every sound producing species had a Q10 value less than 2, indicating they have power over a large range of frequencies (Table 5.3, Ewing 1988). A large frequency spectrum allows the sounds to be received by a variety of predators with different optimal hearing ranges (Masters 1979, 1980). The sonic portions of the signals overlap with the frequency hearing range of birds (Dooling 1991) while the ultrasonic portions could be perceived by bats and even mice (Kirchner and Röschard 1999). There is increasing evidence that bats eat large caterpillars, especially in the tropics (Kalka and Kalko 2006, Wilson and Barclay 2006), which corresponds favourably to my observations of high portions of ultrasound in the signals of Costa Rican caterpillars. The dominant frequency was above $20 \mathrm{kHz}$ in $4 / 5$ Costa Rican species for which I was able to obtain recordings at a high sampling rate (Table 
5.3). Two of those species had peaks solely in the ultrasound, $S$. santarosensis and $P$. drucei (Fig 5.11 and 5.54) suggesting that some species may mainly be targeting bats. Invertebrate predators and parasitoids are also important natural enemies (Pittaway 1993, Tuskes et al. 1996, Tuttle 2007). Although my experiments simulated attacks by a vertebrate predator, I would occasionally use a paintbrush or stiff fishing line to stimulate the caterpillars (e.g. M. sexta and S. pyri), resembling attacks by invertebrates, which could also induce signalling. Though insect predators such as ants lack tympanal ears capable of hearing the sounds produced by these caterpillars (Yack 2004), at least some signals may be transmitted as vibrations through the substrate that can be perceived. Sounds produced by stridulating insects also cause their bodies to vibrate and have been shown to be effective against some spiders (Masters 1979). The low-frequency airborne component may also be heard by spiders (Masters 1979), while the high-frequencies could be detected by insects with ultrasonic sensitive hearing, such as mantids (Yager 1999). Though it is unclear if ants could learn an association between a sound and a defence, they have been shown to learn an association between sounds produced by butterfly caterpillars and danger (e.g. Travassos and Pierce 2000), and mantids have been shown to be able to associate visual cues with noxious defences (Bowdish and Bultman 1993, Prudic et al. 2006). I propose that caterpillar defence sounds are mainly directed at vertebrate predators, primarily birds and bats, and will discuss the hypotheses outlined in the introduction in light of the data collected throughout this thesis.

Startle, Acoustic aposematism and Unprofitability 
Startle sounds, warning signals and unprofitability signals are not easy to separate. All may initially function to startle, or all may warn of some coupled defence, or that the initiated attack is simply a waste of the predator's time. Each idea will be discussed below.

Startle sounds should be conspicuous, presented after crypsis has failed, cause the predator to hesitate allowing for escape and eventually become ineffective as the prey is otherwise undefended (Edmunds 1974, Sargent 1990, Ruxton et al. 2004). Two species that I studied, $A$. juglandis and $S$. abbottii, appear to meet most of these criteria. Both species are cryptic until attacked by a predator (Fig 5.26 and 5.55), and sounds produced by the walnut sphinx are loud ( 82.1 to $86.9 \mathrm{~dB}$ SPL at $10 \mathrm{~cm}$, see Chapter 4 ) while sounds produced by the Abbott's sphinx were accompanied by conspicuous winding thrashing movements. Finally, in both species the sounds were not directly associated with a chemical defence (Fig 5.26j and 5.55j), unlike many other species (see below), satisfying three criteria for startle. Neither caterpillar, however, attempted to escape but this may not be an easy solution for caterpillars as they cannot run or fly away and dropping to the ground can be costly or dangerous (Dethier 1959). In fact, the only saturniids and sphingids of Santa Rosa that have been observed to drop from their hostplants are those that are very polyphagous and would likely not have much trouble finding another suitable food source (Janzen 1984). Habituation to the signals on the part of the predator was not examined, though this was discussed in Chapter 4. The type of predator and the frequency of encountering the startle sounds will determine how each predator responds and whether or not it will habituate to the signals. Birds presented with walnut sphinx caterpillars did not habituate to the signals during the course of the trials, 
but with repeated exposure they may have done so. Future studies should incorporate trials with live predators to gauge the effectiveness of the sounds as potential startle signals as well as to examine the reactions of the predators over a longer period of time to determine if they would eventually habituate.

Aposematic signals are associated with other noxious defences that are used to signal an animal's unprofitability (Poulton 1890). The association between the signal and the defence is learned by the predator so that, over time, the signal alone should be sufficient to deter predation (e.g. Hristov and Conner 2005). Among the Bombycoidea caterpillars I examined, $64.3 \%$ regurgitated in response to attack and an additional $11.9 \%$ possessed a different form of chemical defence (e.g. urticating scoli) (Table 5.4). Of the species that produced sound, $13 / 15$ also regurgitated at some point or produced a chemical secretion (Table 5.5). Of these species, five are candidates for producing aposematic signals as their sounds preceded or accompanied the appearance of their respective noxious defences (Table 5.2). When an animal is presented with the signal prior to receiving a stimulus it will be more likely to remember the association between the two. For example honey bees learned to associate certain colours with a good food source only when they were presented the colour before they were able to feed (reviewed in Carew 2000). Future studies should demonstrate the deterrent properties of suspected noxious defences and closely examine the temporal relationship between the signal and the defence. Also, live predator trials should be performed to evaluate if the signal can be remembered over time.

Sounds produced by these caterpillars were not only associated with a chemical defence, but a variety of other behaviours as well (Table 5.4, 5.5). Many of the surveyed 
caterpillars thrashed, some at high rates, and curled their bodies in response to attack, strategies that might not be offensive to a predator, but that would make the prey item more time consuming to obtain. Avian predators, for example, are constantly moving through the foliage searching for food and must also be on the look-out for their own predators (Kaby and Lind 2003), making foraging a time-sensitive operation. Therefore the sounds produced by bombycoid caterpillars may simply be considered a general signal of unprofitability, telling potential predators to move on to something easier. Both M. pellenia and N. magna were capable of thrashing at high rates (Fig 5.38, 5.51), so their sounds may fall under this category.

\section{Enhanced learning}

The enhanced learning hypothesis says that an additional signal will reinforce an existing association between another signal and a noxious defence. For example, some chicks were able to learn an association between colour and distastefulness more quickly when the stimuli were presented in conjunction with a sound than when the sound was absent (Rowe and Guilford 1999, Rowe 2002). This hypothesis is unlikely to apply to the caterpillars in this thesis since $14 / 15$ sound producing species were cryptically coloured. The one remaining species, $N$. magna, had a bright blue head and yellow and black dorsal pattern and appears to be conspicuously coloured (Fig 5.51), and this hypothesis should be explored further in this species. If sound production is discovered in other conspicuously coloured species, such as some Hyles sp. caterpillars, this hypothesis should be examined in more detail. Tests with predators where the caterpillar is presented 
as silenced or not, over a period of days, may aid in determining the merits of this hypothesis.

\section{Jamming}

Some prey sounds have the ability to interfere with the echolocation calls of bats, such as the sounds emitted by some arctiid moths (Conner 1999, Corcoran et al. 2009) and are considered jamming signals. To qualify as a jamming signal several conditions should be met. First, the sounds must be able to be perceived by bats. Second, the prey item should be capable of hearing the echolocation calls of bats. Finally, the sounds should be produced in response to the echolocation calls or while the predator is calling. Airborne sounds produced by the examined caterpillars meet only the first of these three criteria. The presence of ultrasound in their signals suggests that they can be perceived by bats (Table 5.3). The second and third criteria, however, are not met. Bombycoidea caterpillar do not, to the best of my knowledge possess ears capable of hearing ultrasonic frequencies, and their signals are elicited by physical attack and not another acoustic stimulus, though this was not directly tested. In addition, the structure of their sounds does not resemble the high-duty cycle sounds produced by arctiid moths that have been shown to jam the sonar of bats (Corcoran et al. 2009). Therefore, it is unlikely that bombycoid caterpillar sounds function as jamming signals.

\section{Mimicry}

Based on the similar characteristics of all the signals (Table 5.3), it is possible that there is either Müllerian or Batesian mimicry within the group; in fact this could be true 
of all disturbance sounds since they are all similar (Masters 1979, 1980). I do not believe, however that the sounds produced by these caterpillars are mimicking the sounds produced by any other specific animals. It would be difficult to investigate the possibility of acoustic mimicry because even in a mimic the sounds may still function to startle a predator.

Alarm/recruitment of con- and heterospecifics

Alarm and recruitment sounds function to alert others to a danger and/or gather individuals to overcome a potential predator (Bradbury and Vehrencamp 1998). To function as an alarm or recruitment call, con- or heterospecifics should be located close enough to the sound source to be able to respond to it, and also be physically able to hear the sounds. Bombycoidea caterpillars are not eusocial, and most are solitary feeders in their late instars (Tuttle 2007, Lampe 2010) and thus are not normally in close proximity to one another. They also, to the best of my knowledge, do not possess ears capable of hearing airborne sounds and could therefore not respond to another caterpillar in danger. Finally, there have been no reports that I am aware of that have investigated any kind of mutualism of these caterpillars with other species, like ants, that could come to their aid. Consequently, I believe it is unlikely that bombycoid signals function as alarm or recruitment calls.

Bombycoidea caterpillar sounds likely fall into two of the general categories outlined above, startle and aposematism, but could also serve a number of purposes. In fact, all sounds may initially startle a hunting predator and, as the predator gains experience and learns an association with a defence, may later be interpreted as a warning 
sound (Bailey 1991). Future studies should attempt to incorporate interactions between the caterpillars and natural predators, as well as the reactions of the predators to the sounds, to tease apart differences in function, if any exist.

\section{Why don't all caterpillars make defensive sounds?}

As previously mentioned, to date, sound production has only been observed in species of the Bombycoidea. This is supported by my data set, although it is important to recognize that my survey of non- bombycoid species was very limited. However, to the best of my knowledge, there are no reports of sound production in other caterpillars in the literature, which lends support to my observations since many other large species have been studied and reared by amateur collectors, naturalists and scientists. Consequently, I assume that if sound production is not restricted to this superfamily, it is probably most common in this group. This leads to two interesting questions: Why is sound production more common in Bombycoidea than other Lepidoptera superfamilies?, and Why, within the group, do some species produce sounds while others do not? Factors that may drive the evolution of a given defence fall into three main categories, which will be discussed below: existing preconditions (such as size, chemical defence, mandible morphology and head morphology), selection pressures that cause an increase in fitness (such as predation) and other trade-offs or costs/benefits (such as investment in other defences).

Why is sound production most common in the Bombycoidea?

If, as a prey item, your defensive strategy includes the use of airborne sounds, then you should be able to produce sounds that are sufficiently loud for a predator to hear. In insects, there are size constraints that limit the amplitude of the sound that can be 
produced (reviewed in Bennet-Clark 1998), and this will apply to caterpillars as well. The families Sphingidae and Saturniidae have caterpillars that are described as "large to enormous" with species that can exceed $10 \mathrm{~cm}$ in length (Wagner 2005), and should therefore be capable of producing audible sounds. Indeed, the sounds of many caterpillars in this survey were perfectly audible by an unaided ear in a quiet room for distances up to two metres. Therefore, the overall size of the caterpillars in this group may be a contributing factor in the evolution of sound production. Future studies should continue to test a variety of large species in different groups to determine if acoustic signalling is, in fact, unique to the Bombycoidea.

Discussions surrounding the evolution of aposematism state that conspicuous coloration to advertise unprofitability likely evolved from an originally cryptic and defended morph (reviewed in Ruxton et al. 2004). Of the bombycoid caterpillars examined in this survey $76 \%$ either regurgitated in response to attack or possessed a chemical defence (Table 5.4). The majority of these species were also cryptically coloured (see plates). Perhaps, once discovered, the cost of advertertising the existing defence outweighed the costs of a prolonged attack (i.e. fatal injury). As stated above, the larvae in this superfamily are large. Since there is no correlation between size and detectability in cryptic species, as there is for conspicuously coloured species (Mand et al. 2007) it is possible that evolution favoured the development of an acoustic signal in this group. Further tests with other large caterpillars (defended and undefended) that exhibit a variety of coloration patterns should be carried out in order to determine if either of these features (or combinations thereof) could be used as predictors for the development of sound production within a given taxon. 
Why don't all Bombycoidea caterpillars produce sounds?

As seen throughout this chapter, there are many species of Bombycoidea caterpillars that do not use sound production as part of their defensive strategy. Evidently there are more factors at work within the group than size and coloration. In order to make hypotheses regarding which species will make sounds and which will remain silent it is necessary to examine differences in morphology (existing preconditions), selection pressures (predation) and behaviour (investment in other defences).

Size

The differences in the size of individual species within the superfamily may help explain the existence of sound production in some species and not others. Though in general, bombycoid caterpillars are considered large, there is some variation among the different species. Maximum reported sizes for sound producing species ranged from 6-13 $\mathrm{cm}$ in length (average $8.23 \pm 2.05 \mathrm{~cm}$ ) and silent species ranged from $5-12 \mathrm{~cm}$ in length (average $7.60 \pm 2.00 \mathrm{~cm}$ ) (Table 5.2). These results indicate that the size of a given species within the superfamily does not appear to be a precondition for producing sound (at least using maximum length as an indicator of size). Future studies should examine other characteristics of size, such as mass for example, to see if any trends exist.

Mandible morphology, head shape and musculature

One possibility for the observations that some species produce sound while others do not is the structure of the mandibles, at least for those species that use their mandibles to produce sound (i.e. clicking or stridulation). The three general types of mandible 
morphology observed among the different species were ridged and serrated, serrated, and smooth. Of the species for which there was information, ridged and serrated mandibles were found in $6 / 7$ species that clicked and $6 / 20$ silent species. Serrated mandibles were observed in $2 / 3$ species that stridulated and $4 / 20$ silent species. Finally, smooth mandibles were found in $1 / 7$ species that clicked, $1 / 3$ species that stridulated and $10 / 20$ species that were silent. It is not surprising that no clear trends are evident since it has been shown that mandible morphology is closely associated with diet (Bernays and Janzen 1988, reviewed in Clissold 2007) and this can be similar between sound producing and silent species (e.g. A. polyphemus and D. rubicunda can both be found on maple). However, I do suggest that the ability to click stems from having ridged mandibles (although there is one exception) and that the ability to stridulate requires a lack of ridges. Further examination of the mandible morphologies in species that use these structures to produce sound will determine the validity of my suggestion.

Another morphological feature examined in this thesis was head shape. Heads could be categorized into three general shapes, oval, triangular or rounded. No species with oval heads made sounds, and only one species with a triangular head made sound (non-mandible mechanism, A. juglandis). Twelve species with rounded heads produced sound, 10 that used a mandible mechanism and two that did not (rasping, S. abbottii and N. magna). Though there were also non-sound producing species with round heads (e.g. A. atlas, A. io), perhaps a round head is indicative of a capacity to produce sound using the mandibles (i.e. other head shapes lack this capacity). Head capsule morphology is determined by the sizes and attachment points of the muscles that control the mandibles (reviewed in Clissold 2007). These muscles can be made up of different proportions of 
fast and slow muscle fibres and can be controlled by two types of neurons (fast and slow) that allow the mandibles to move in different ways (reviewed in Clissold 2007). This can be important in different foraging techniques, for example, ants that used a predatory strategy had long heads and fast muscle fibres, whereas ants that cracked seeds or harvested leaves had short heads and slow muscle fibres (references in Clissold 2007). I hypothesize that differences in the muscle fibres and neuronal control of the adductor muscle will determine if a caterpillar is capable of producing sound with its mandibles. It has been shown that $M$. sexta adductor muscles consist solely of the fast muscle fibre type (references in Clissold 2007). If this is true of all sound producing species, this hypothesis may have some merit. Future studies should involve gross dissection and histological examinations to determine if there are structural differences in the make-up of the muscles that control the mandibles in both silent and sound producing species.

Predation

Gathering data on specific predators is difficult as it requires observations of natural interactions. Therefore I can only speculate on the possible selection pressures that might explain why some species produce sounds while others do not. Low light conditions may favour the evolution of an acoustic signal as predators that hunt at these times are less reliant on vision. Many of the caterpillars in this survey remain hidden and motionless during the day and actively move through their hostplant to feed at night. Perhaps this nocturnal activity is more prevalent in sound producing caterpillars. For example, the Abbott's sphinx caterpillar, a sound producer, can travel up to two meters from its day-time resting place to feed at the leaves (Heinrich 1979). Once there, these 
caterpillars, which are nearly invisible during the day, are very easy to find with the aid of a flashlight (Wagner 2005). Additionally, areas of dense forest can have low light levels, and again, many species in this study would be found in such areas. In this case even a visually hunting predator may require additional information to make foraging decisions and an acoustic signal could be beneficial. Future studies should attempt to characterize low light activities in both silent and sound producing species, as well as reactions of predators to sounds in low light conditions.

Trade-offs (cost/benefit)

Existing behaviours or morphologies may limit an investment in additional defences since there may be energetic or other costs associated with their development. For example, non-sound producing species displayed a number of other defensive behaviours including chemical defense, thrashing and biting. Perhaps these defences are stronger in silent species than in their sound producing confamiliars and are sufficient on their own to deter predation. Another situation where the development of sound may be too 'expensive' (or unnecessary) is in those species that already have an existing investment in a visual display. Visual displays may stop an attack before it begins, whereas a sound will only be able to stop subsequent attacks after the initial one. A recent study of Papilio caterpillars found that they were more likely to be conspicuously coloured when they fed on plants with narrow leaves and cryptic when they fed on plants with broad leaves (Prudic et al. 2007). The caterpillars on narrow leaves were likely to be spotted despite their coloration strategy as there was less material to hide them, so an investment in a conspicuous visual appearance makes sense. It would be interesting to 
test if the same pattern existed in Bombycoidea species and if those species that were conspicuously coloured on narrow leaved plants produced sounds.

All of the above suggestions would benefit from studies on the natural histories and predator interactions of individual species. The reactions of predators to the sounds produced by these caterpillars are also largely unknown apart from the preliminary experiments I did with yellow warblers in Chapter 4. It is clear, however, that no single trait can determine whether or not a species of caterpillar will produce sounds as part of its defensive strategy. 


\section{GENERAL CONCLUSIONS}

The goal of my thesis was to explore the diversity of sound production in a group of caterpillars where, previously, there had been several anecdotal reports from naturalist observations but only one study that documented an experimental example ( $A$.

polyphemus, Brown et al. 2007). Over the course of my thesis I examined the defensive behaviour of 42 species of Bombycoidea caterpillars and 11 outgroup species. Sound production was found in 15 species of bombycoids and no doubt others will be unveiled in future research. Caterpillar defences have been widely studied, contributing to our understanding of the sequestration of plant chemicals, the evolution of aposematic coloration and the learning processes of predators, among others. Now research will need to consider sounds in the defence of these species and think beyond visually biased predators, especially considering the amount of ultrasound in the signals that may be focused towards animals like bats. Indeed, my results on the large diversity of airborne sound production in Bombycoidea caterpillars taken together with the increasing evidence of vibratory communication in other species, as well as developments in the area of detection of airborne sounds, caterpillars can no longer be thought of as lacking an acoustic sense. Quite the opposite, it appears that acoustics could play a key role in their survival. Considering caterpillars have high economic and ecological importance, research on their acoustic communication is highly significant. My research has not only revealed insights into the diversity of the distribution, mechanisms and function of sound production, but has also opened opportunities for future research, including: the examination of taxonomic groups where sound production is mapped using phylogenetic 
analysis to determine likely evolutionary scenarios; the effects of sounds on predators, who responds and how do they respond?; histological and morphological studies to determine the inner-workings of sound producing mechanisms, especially for rasping sounds which may represent a mechanism used by only one other insect (to the best of my knowledge); and further examinations into the arsenal of defences in caterpillars to answer questions about why some species do and do not use sounds. The results of my thesis reveal the extreme diversity of a mode of communication that most people would doubt exists for caterpillars and will provide a foundation for future comprehensive and interesting research. 


\section{REFERENCES}

Alexander, R. D. (1960) Communicative mandible snapping in Acrididae. Science 132: $152-153$

American Ornithologists' Union. (1998) Check-list of North American Birds. 7th edition. Washington D.C.: American Ornithologists' Union.

Asuncion-Uchi, M., El Shawa, H., Martin, T. and Fuse, M. (2010) Different actions of ecdysis-triggering hormone on the brain and ventral nerve cord of the hornworm Manduca sexta. Gen Comp Endocr 166: 54-65

Bacher S., Casas J. and Dorn S. (1996) Parasitoid vibrations as potential releasing stimulus of evasive behaviour in a leafminer. Physiol Entomol 21: 33-43

Bailey, W. J. (1991) Acoustic Behaviour of Insects: an Evolutionary Perspective. New York: Chapman and Hall.

Bailey, W. J. (2003) Insect duets: underlying mechanisms and their evolution. Physiol Entomol 28: 157-174

Barber, J. R. and Conner, W. E. (2007) Acoustic mimicry in a predator-prey interaction. P Natl Acad Sci USA 104: 9331-9334

Bates, D. L. and Fenton, M. B. (1990) Aposematism or startle? Predators learn their responses to the defenses of prey. Can J Zool 68: 49-52

Bennet-Clark, H. C. (1998) Size and scale effects as constraitnts in insect sound communication. Phil Trans $R$ Soc Lond B 353: 407-419

Bernays, E. A. and Janzen, D. H. (1988) Saturniid and sphingid caterpillars: two ways to eat leaves. Ecology 69: 1153-1160

Bethune, C. J. S. (1868) A musical larva. Can Entomol 1: 41

Blondheim, S. A. and Frankenberg, E. (1983) 'Protest' sounds of a grasshopper: predator deterrent signal? Psyche 90: 387-394

Bowdish, T. I. and Bultman, T. L. (1993) Visual cues used by mantids in learning aversion to aposematically colored prey. Am Midl Nat 129: 215-222

Bowen, J. L., Mahony, S. J., Mason, A. C. and Yack, J. E. (2008) Vibration-mediated territoriality in the warty birch caterpillar Drepana bilineata. Physiol Entomol 33: $238-250$ 
Bowers, M. D. (2003) Hostplant suitability and defensive chemistry of the Catalpa sphinx, Ceratomia catalpae. J Chem Ecol 29: 2359-2367

Bradbury, J. W. and Vehrencamp, S. L. (1998) Principles of Animal Communication. Sunderland: Sinauer Associates.

Broughton, W. B. (1963) Method in bio-acoustic terminology. In Acoustic Behaviour of Animals (ed. R. G. Busnel), pp. 3-24. New York: Elsevier.

Brower, J. Z. and Brower, L. P. (1965) Experimental studies of mimicry. 8. Further investigations of honeybees (Apis mellifera) and their dronefly mimics (Eristalis spp.). Am Nat 99: 173-187

Brown, S. G. (2006) Clicking caterpillars: acoustic aposematism in Antheraea polyphemus and other Bombycoidea. M.Sc. thesis, Carleton University, Ottawa, Canada.

Brown, S. G., Boettner, G. H., and Yack, J. E. (2007) Clicking caterpillars: acoustic aposematism in Antheraea polyphemus and other Bombycoidea. J Exp Biol 210: 993-1005

Bura, V. L., Fleming, A. J. and Yack J. E. (2009) What's the buzz? Ultrasonic and sonic warning signals in caterpillars of the great peacock moth (Saturnia pyri). Naturwissenschaften 96: 713-718

Busnel, R. G. and Dumortier, B. (1959) Vérification par des methods d'analyse acoustique des hypotheses sur l'origine du cri du sphinx Acherontia atropos (Linné). B Soc Entomol Fr 64: 44-58

Carew, T. J. (2000) Behavioral Neuobiology: The Cellular Organization of Natural Behaviour. Sunderland: Sinauer Associates, Inc.

Casas, J., Bacher, S., Tautz, J., Meyhöfer, R. and Pierre, D. (1998) Leaf vibrations and air movements in a leafminer-parasitoid system. Biol Control 11: 147-153

Castellanos, I. and Barbosa, P. (2006) Evaluation of predation risk by a caterpillar using substrate-borne vibrations. Anim Behav 72: 461-469

Clissold, F. J. (2007) The biomechanics of chewing and plant fracture: mechanisms and implications. Adv Insect Physiol 34: 317-372

Conner, W. E. (1999) 'Un chant d'appel amoureux': acoustic communication in moths. $J$ Exp Biol 202: 1711-1723

Corcoran, A. J., Barber, J. E. and Conner W. E. (2009) Tiger moth jams bat sonar. Science 325: 325-327 
Covell, C. V. (2005) A Field Guide to Moths of Eastern North America. Martinsville: Virginia Museum of Natural History.

Crane, J. (1966) Combat, display and ritualization in fiddler crabs (Ocypodidae, genus Uca). Philos T R Soc B 251: 459-472

Deml, R. (2001) Influence of food and larval age on the defensive chemistry of Saturnia pyri. Z Naturforsch 56:89-94

Deml, R. and Dettner, K. (1993) Biogenic amines and phenolics characterize the defensive secretion of saturniid caterpillars (Lepidoptera: Saturniidae): a comparative study. J Comp Physiol B 163:123-132

Deml, R. and Dettner, K. (1994) Attacus atlas caterpillars (Lep., Saturniidae) spray an irritant secretion from defensive glands. J Chem Ecol 20: 2127-2138

Deml, R. and Dettner, K. (1995) Effects of emperor moth larval secretions, hemolymph, and components on microorganisms and predators. Entomol Exp Appl 76: 287293

Deml, R. and Dettner, K. (2002) Morphology and classification of larval scoli of Saturniinae and Hemileucinae (Lepidoptera: Saturniidae). J Zool Syst Evol Research 40: 82-91

Dethier, V. G. (1959) Food-plant distribution and density and larval dispersal as factors affecting insect populations. Can Entomol 92: 581-596

DeVries, P. J. (1990) Enhancement of symbioses between butterfly caterpillars and ants by vibrational communication. Science 248: 1104-1106

Dooling, R. J. (1991) Hearing in birds. In The Evolutionary Biology of Hearing (eds. D. Webster, R. Fay and A. Popper), pp. 545-560. New York: Springer-Verlag.

Dumortier, B. (1963) Morphology of sound emission apparatus in Arthropoda. In Acoustic Behaviour of Animals (ed. R. G. Busnel), pp. 277-338. New York: Elsevier.

Dyer, L. A. (1997) Effectiveness of caterpillar defences against three species of invertebrate predators. J Res Lepidoptera 34: 48-68

Edmunds, M. (1974) Defence in Animals; A Survey of Anti-predator Defences. New York: Logman Group Ltd.

Eisner, T., Rossini, C., González, A. and Eisner, M. (2004) Chemical defense of an opilionid (Acanthopachylus aculeatus). J Exp Biol 207: 1313-1321 
Ewing, A. M. (1989) Arthropod Bioacoustics: Neurobiology and Behavior. New York: Cornell University Press.

Federley, H. (1905) Sound produced by Lepidopterous larvae. J NY Entomol Soc 13: 109110

Field, L. H. and Matheson, T. (1998) Chordotonal organs of insects. Adv Insect Physiol 27: $1-228$

Fletcher, L. E. (2007) Vibrational signals in a gregarious sawfly larva (Perga affinis): groop coordination or competitive signalling? Behav Ecol Sociobiol 61: 18091821

Fletcher, L. E., Yack, J. E., Fitzgerald, T. D. and Hoy, R. R. (2006) Vibrational communication in the cherry leaf roller caterpillar Caloptilia serotinella (Gracillarioidea: Gracillariidae). J Insect Behav 19: 1-18

Fraser, J. and Nelson, M. C. (1982) Frequency modulated courtship song in a cockroach. Anim Behav 30: 627-628

Gaddis, P. (1980) Mixed flocks, Accipiters, and antipredator behavior. Condor 82: 348349

Gentry, G. L. and Dyer, L. A. (2002) On the conditional nature of neotropical caterpillar defenses against their natural enemies. Ecology 83: 3108-3119

Goerlitz, H. R., Greif, S. and Siemers, B. M. (2008) Cues for acoustic detection on prey: insect rustling sounds and the influence of walking substrate. $J \operatorname{Exp}$ Biol 211: 2799-2806

Grant, J. B. (2006) Diversification of gut morphology in caterpillars is associated with defensive behavior. $J$ Exp Biol 209: 3018-3024

Greeney, H. F., DeVries, P. J., Penz, C. M., Granizo-T, R. B., Connahs, H., Stireman, J. O., Walla, T. R. and Dyer, L. A. (2009) The early stages and natural history of Antirrhea adoptive porphyrosticta (Watkins, 1928) in eastern Ecuador (Lepidoptera: Nymphalidae: Morphinae). J Insect Sci 9: article 30

Greenlee, K. J. and Harrison, J. F. (2005) Respiratory changes throughout ontogeny in the tobacco hornworm caterpillar, Manduca sexta. J Exp Biol 208: 1385-1392

Gulbitti-Onarici, S., Zaidi, M. A., Taga, I., Ozcan, S. and Altosaar, I. (2009) Expression of Cryl Ac in transgenic tobacco plants under the control of a wound-inducible promoter (AoPR1) isolated from Asparagus officinalis to control Heliothis virescens and Manduca sexta. Mol Biotechnol 42: 341-349 
Hauglund, K., Hagen, S. B. and Lampe, H. M. (2006) Responses of domestic chicks (Gallus gallus domesticus) to multimodal aposemtic signals. Behav Ecol 17: 392-398

Haskell, P. T. (1961) Insect Sounds. London: H. F. \& G. Witherby Ltd.

Haskell, P. T. (1974) Sound production. In The Physiology of Insecta, $2^{\text {nd }}$ Ed. (ed. M. Rockstein), pp. 353-410. New York: Academic Press Inc.

Heinrich, B. (1979) Foraging strategies of caterpillars: leaf damage and possible predator avoidance strategies. Oecologia 42: 325-337

Heinrich, B. and Collins, M. (1983) Caterpillar leaf damage and the game of hide-andseek with birds. Ecology 64: 592-602

Hoy, R. R. and Robert, D. (1996) Tympanal hearing in insects. Ann Rev Entomol 41: 433-450

Hristov, N. I. and Conner, W. E. (2005) Sound strategy: acoustic aposematism in the battiger moth arms race. Naturwissenschaften 92: 164-169

Jansson, A. and Vuoristo, T. (1979) Significance of stridulation in larval Hydropsychidae (Trichoptera). Behaviour 71: 167-185

Janzen, D. H. (1984) Two ways to be a big tropical moth: Santa Rosa saturniids and sphingids. In Oxford Surveys of Evolutionary Biology (ed. R. Dawkins and M. Ridley), pp. 85-140. New York: Oxford University Press

Janzen, D. H. and Hallwachs, W. (2009) Dynamic database for an inventory of the macrocaterpillar fauna, and its food plants and parasitoids, of Area de Conservacion Guanacaste (ACG), northwestern Costa Rica (nn-SRNP-nnnnn voucher codes) $<$ http://janzen.sas.upenn.edu $>$.

Kaby, U. and Lind, J. (2003) What limits predator detection in blue tits (Parus caeruleus): posture, task or orientation? Behav Ecol Sociobiol 54: 534-538

Kalka, M. and Kalko, E. K. V. (2006) Gleaning bats as underestimated predators of herbivorous insects: diet of Micronycteris microtis (Phyllostomidae) in Panama. J Trop Ecol 22: 1-10

Kawahara, A. Y., Mignault, A. A., Regier, J. C., Kitching, I. J. and Mitter, C. (2009) Phylogeny and biogeography of hawkmoths (Lepidoptera: Sphingidae): Evidence from five nuclear genes. PLOS One 4: e5719 
Kingsolver, J. G. (2007) Variation in growth and instar number in field and laboratory Manduca sexta. Proc R Soc B 274: 977-981

Kirchner, W. H. (1997) Acoustical communication in social insects. In: Orientation and Communication in Arthropods (ed. M. Lehrer), pp 273-300. Basel Switzerland: Birkhauser Verlag.

Kirchner, W. H. and Röschard, J. (1999) Hissing in bumblebees: an interspecific defence signal. Insectes Soc 46: 239-243

Kitching, I. J. and Cadiou, J-M. (2000) Hawkmoths of the World. Ithaca: Cornell University Press.

Kristensen, N. P. and Skalski, A. W. (1998) Phylogeny and palaeontology. In Handbook of Zoology Volume IV: Arthropoda: Insecta, Part 35 Lepidoptera, Moths and Butterflies, pp 7-26. Berlin: Walter de Gruyter.

Lampe, R. E. J. (2010) Saturniidae of the World. München: Verlag Dr. Friedrich Pfeil

Lima, S.L. (1993) Ecological and evolutionary perspectives on escape from predatory attach: a survey of North American birds. Wilson Bull 105:1-47

Low, C. (2008) Seismic behaviours of a leafminer, Antispila nysaefoliella (Lepidoptera: Heliozelidae). Florida Entomol 91: 604-609

Lowther, P. E., Celada, C., Klein, N. K. , Rimmer, C. C. and Spector, D. A. (1999) Yellow Warbler (Dendroica petechia), The Birds of North America Online (ed. A. Poole). Ithaca: Cornell Lab of Ornithology; Retrieved from the Birds of North America Online: http://bna.birds.cornell.edu/bna/species/454

Machado, G. and Freitas, A. V. (2001) Larval defence against ant predation in the butterfly Smyrna blomfildia. Ecol Entomol 26: 436-439

Mand, T., Tammau, T. and Mappes, J. (2007) Size-dependent predation risk in cryptic and conspicuous insects. Evol Ecol 21: 485-498

Mansingh, A. (1972) Developmental response of Antheraea pernyi to seasonal changes in oak leaves from two localities. J Insect Physiol 18: 1395-1401

Marples, N. M., van Veelen, W. and Brakefield, P. M. (1994) The relative importance of colour, taste and smell in the protection of an aposematic insect Coccinella septempunctata. Anim Behav 48: 967-974

Marquis, R. J. (1984) Natural history of a daytime-flying saturniid: Automeris phrynon Druce (Lepidoptera: Saturniidae: Hemilucinae). J Kansas Entomol Soc 57: 529531 
Mason, A. (1996) Territoriality and the function of song in the primitive acoustic insect Cyphoderris monstrosa (Orthoptera: Haglidae). Anim Behav 51: 221-224

Masters, W. M. (1979) Insect disturbance stridulation: its defensive role. Behav Ecol Sociobiol 5: 187-200

Masters, W. M. (1980) Insect disturbance stridulation: characterization of airborne and vibrational components of the sound. J Comp Physiol 135: 259-268

Mead, T. L. (1869) Musical larvae. Can Entomol 1: 47

Miller, P. L. (1974) Respiration- Aerial gas transport. In The Physiology of Insecta, Second Edition (ed. M. Rockstein), pp. 346-402. New York: Academic Press.

Miller, L. A. and Surlykke, A. M. (2001) How some insects detect and avoid being eaten by bats: Tactics and counter-tactics of prey and predator. Bioscience 51:570-581

Miller, J. C., Janzen, D. H. and Hallwachs, W. (2006) 100 Caterpillars: Portraits from the Tropical Forests of Costa Rica. Cambridge: The Belknap Press of Harvard University Press.

Minet, J. and Surlykke, A. (2003) Auditory and sound producing organs. In Handbook of Zoology, Vol. IV (Arthropoda: Insecta. Lepidoptera, Moths and Butterflies, Vol. 2) (ed. N. P. Kristensen), pp. 289-323. New York: WG de Gruyter.

Minnich, D. E. (1936) The responses of caterpillars to sounds. $J$ Exp Zool 72: 439-453

Mohl, B. and Miller, L. A. (1976) Ultrasonic clicks produced by the peacock butterfly: A possible bat-repellent mechanism. J Exp Biol 64: 639-644

Morales, M. A., Barone, J. L. and Henry, C. S. (2008) Acoustic alarm signalling facilitates predator protection of treehoppers by mutualist ant bodyguards. Proc $R$ Soc B 275: 1935-1941

Mukerji, D. (1929) Sound production by larva of Cybister (Dytiscidae). J Bombay Nat Hist Soc 33: 653-655

Nelson, M. C. (1979) Sound production in the cockroach, Gromphadorhina portentosa: the sound-producing apparatus. J Comp Physiol 132: 27-38

Nishida, R. (2002) Sequestration of defensive substances from plants by Lepidoptera. Annu Rev Entomol 47: 57-92

Oehkle, B. (2009) Sphingidae of the Americas. Accessed online at $<\mathrm{http}: / / \mathrm{www}$. silkmoths.bizland.com/danjansphinx.htm> on May 7, 2010 
Packard, A. S. (1904) Sound produced by a Japanese saturnian caterpillar. J N Y Entomol Soc 12: $92-93$

Papachristoforou, A., Sueur, J., Rortais, A., Angelopoulos, S., Thrasyvoulou, A. and Arnold, G. (2008) High frequency sounds produced by Cyprian honeybees Apis mellifera cypria when confronting their predator, the Oriental hornet Vespa orientalis. Apidologie 39: 468-474

Pearce, W. T. (1886) Stridulation of pupae of Acherontia atropos. Entomologist 19: 44

Peigler, R. S. (1989) A revision of the Indo-Australian genus Attacus. California: The Lepidoptera Research Foundation, Inc.

Pittaway, A. R. (1993) Hawkmoths of the Western Palaearctic. England: Harley Books.

Pittaway, A. R. (2010) Saturniidae of Europe. Accessed online at $<\mathrm{http}$ //tpittaway.tripod.com/silk/satlist.htm> on May 7, 2010

Pittaway, A. R. and Kitching, I. J. (2010) Sphingidae of the Eastern Palaearctic. Accessed online at <http://tpittaway.tripod.com/china/> on May 7, 2010

Poulton, E. B. (1890) The Colours of Animals: Their Meaning and Use Especially Considered in the Case of Insects. London: Keegan Paul, Trench, Trübner.

Prudic, K. I., Skemp, A. K. and Papaj, D. R. (2006) Aposematic coloration, luminance contrast, and the benefits of conspicuousness. Behav Ecol 18: 41-46

Prudic, K. I., Oliver, J. C. and Sperling, A. H. (2007) The signal environment is more important than diet or chemical specialization in the evolution of warning coloration. PNAS 104: 19381-19386

Randall, J. A. (2001) Evolution and function of drumming as communication in mammals. Amer Zool 41: 1143-1156

Rashed, A., Khan, M. I., Dawson, J. W., Yack, J. E. and Sherratt, T. N. (2009) Do hoverflies (Diptera: Syrphidae) sound like the Hymenoptera they morphologically resemble? Behav Ecol 20: 396-402

Ratcliffe, J. M. and Nydam, M. L. (2008) Multimodal warning signals for a multiple predator world. Nature 455: 96-99

Reed, E. B. (1868) A musical larva. Can Entomol 1: 40

Regier, J. C., Cook, C. P., Mitter, C. and Hussey, A. (2008a) A phylogenetic study of the 'bombycoid complex' (Lepidoptera) using five protein-coding nuclear genes, 
with comments on the problem of macrolepidopteran phylogeny. Syst Entomol 33: $175-189$

Regier, J. C., Grant, M. C., Mitter, C., Cook, C. P., Peigler, R. S. and Rougerie, R. (2008b) Phylogenetic relationships of wild silkmoths (Lepidoptera: Saturniidae) inferred from gour protein-coding nuclear genes. Syst Entomol 33: 219-288

Roces, F. and Hölldobler, B. (1996) Use of stridulation in foraging leaf-cutting ants: mechanical support during cutting or short-range recruitment signal? Behav Ecol Sociobiol 39: 293-299

Rostás, M. and Blassmann, K. (2009) Insects had it first: surfactants as a defence against predators. Proc R Soc B 276: 633-638

Rothschild, M., Aplin, R., Baker, J. and Marsh, N. (1979) Toxicity induced in the tobacco horn-worm (Manduca sexta L.) (Sphingidae, Lepidoptera). Nature 280: 487-488

Rougeot, P-C. (1971) Les bombycoïdes (Lepidoptera - Bombycoïdea) de l'Europe et du Bassin Méditerranéen. Paris, France: Masson et Compagnie Éditeurs

Rowe, C. (2002) Sound improves visual discrimination learning in avian predators. Proc $R$ Soc B 269: 1353-1357

Rowe, C. and Guilford, T. (1999) The evolution of multimodal warning displays. Evol Ecol 13: 655-671

Ruxton, G. D., Sherratt, T. N. and Speed, M. P. (2004) Avoiding attack: the evolutionary ecology of crypsis, warning signals and mimicry. New York: Oxford University Press

Sanborn, F. G. (1868) Musical larvae. Can Entomol 1: 48

Sargent, T. D. (1990) Startle as an anti-predator mechanism, with special reference to the underwing moths, (Catocala). In Insect defenses: adaptive mechanisms and strategies of prey and predators (eds. D. L. Evans and J. O. Schmidt), pp 229250. Albany: State University of New York Press.

Savela, M. (2009) Bombycoidea. Accessed online at $<$ http://www.nic.funet.fi/pub/sci/bio/life/insecta/lepidoptera/ditrysia/bombycoide a/index.html $>$ on May 7, 2010

Schwartzkopff, J. (1955) On the hearing of birds. Auk 72: 340-347

Scoble, M. J. (1995) The Lepidoptera. Form function and diversity. Oxford University Press, New York. 
Scott, J. L., Kawahara, A. Y., Skevington, J. H., Yen, S. H., Sami, A., Smith, M. L. and Yack, J. E. (2010a) The evolutionary origins of ritulaized acoustic signals in caterpillars. Nature Communications 1: article number 4

Scott, J. L., Matheson, S. M. and Yack, J. E. (2010b) Variation on a theme: vibrational signalling in caterpillars of the rose hook-tip moth, Oreta rosea. J Insect Sci 10: article 54

Sen Sarma, M., Fuchs, S., Weber, C. and Tautz, J. (2002) Worker piping triggers hissing for coordinated colony defence in the dwarf honeybee Apis florea. Zoology 105: 215-223

Spangler, H. G. (1988) Moth hearing, defense, and communication. Annu Rev Entomol 33: $59-81$

Stamp, N. E. (1986) Physical constraints of defense and response to invertebrate predators by pipevine caterpillars (Battus philenor: Papilionidae). J Lepid Soc 40: $191-205$

Stamp, N. E. (2001) Effects of prey quantity and quality on predatory wasps. Ecol Entomol 26: 292-301

Stumpner, A. and von Helversen, D. (2001) Evolution and function of auditory systems in insects. Naturwissenschaften 88: 159-170

Sueur, J. and Aubin, T. (2006) When males whistle at females: complex FM acoustic signanls in cockroaches. Naturwissenschaften 93: 500-505

Sugi, S. (1987) Larvae of Larger Moths in Japan. Tokyo: Kodansha Co. Ltd.

Sugiura, S. and Yamazaki, K. (2006). The role of silk threads as lifelines for caterpillars: pattern and significance of lifeline-climbing behavior. Eco. Entomol 31: 52-57

Tautz, J. and Markl, H. (1978) Caterpillars detect flying wasps by hairs sensitive to airborne vibration. Behav Ecol Sociobiol 4: 101-110

Taylor, C. (2009) Hearing in larvae of the monarch butterfly, Danaus plexippus, and selected other Lepidoptera. M.Sc. Thesis, Carleton University, Ottawa, Canada.

Thaler, J. S. and Griffin, C. A. M. (2008) Relative importance of consumptive and nonconsumptive effects of predators on prey and plant damage: the influence of herbivore ontogeny. Entomol Exp Appl 128: 34-40

Thurston, R. and Prachuabmoh, O. (1971) Predation by birds on tobacco hornworm larvae infesting tobacco. J Econ Entomol 64: 1548-1549 
Tinbergen, N. (1952) "Derived" activities; their causation, biological significance, origin, and emancipation during evolution. $Q$ Rev Biol 27: 1-32

Travassos, M. A. and Pierce, N. E. (2000) Acoustics, context and function of vibrational signaling in a lycaenid butterfly-ant mutualism. Anim Behav 60: 13-26

Troubridge, J. T. and Lafontaine, J.D. (2004) Moths of Canada. Accessed online at $<$ http://www.cbif.gc.ca/spp_pages/misc_moths/phps/macroindex_e.php>, June 14, 2010.

Tuskes, P. M., Tuttle, J. P. and Collins, M. M. (1996) The Wild Silkmoths of North America. Ithaca: Cornell University Press.

Tuttle, J. P. (2007) The Hawk Moths of North America. Washington DC: The Wedge Entomological Research Foundation.

Vallin, A., Jakobsson, S., Lind, C. and Wiklund, C. (2005) Prey survival by predator intimidation: an experimental study of peacock butterfly defence against blue tits. Proc R Soc B 272: 1203-1207

Vallin, A., Jakobsson, S. and Wiklund, C. (2007) "An eye for an eye?" - on the generality of the intimidating quality of eyespots in a butterfly and a hawkmoth. Behav Ecol Sociobiol 61: 1419-1424

Wagner, D. L. (2005) Caterpillars of North America. Princeton: Princeton University Press.

Walters, E.T., Illich, P.A., Weeks, J.C. and Lewin, M.R. (2001) Defensive responses of larval Manduca sexta and their sensitization by noxious stimuli in the laboratory and field. J Exp Biol 204: 457-469

Westneat, M. W., Betz, O., Blob, R. W., Fezzaa, K., Cooper, J. W. and Lee, W-K. (2003) Tracheal respiration in insects visualized with synchrotron $\mathrm{X}$-ray imaging. Science 299: 558-560

Wilson, J. M. and Barclay, R. M. R. (2006) Consumption of caterpillars by bats during an outbreak of western spruce budworm. Am Midl Nat 155: 244-249

Yack, J. E. (2004) The structure and function of auditory chordotonal organs in insects. Micro Res Techniq 63: 315-337

Yack, J. E. and Dawson, J. (2008) Insect Ears. In: The Senses: A Comprehensive Reference, Volume 3 (eds. R. R. Hoy, G. M. Shepherd, A. I. Basbaum, A. Kaneko and G. Westheimer), pp.35-54. San Diego: Academic Press. 
Yack, J. E. and Hoy, R. R. (2004) Hearing. In Encyclopedia of Insects (eds. V. H. Resh and R. Cardé), pp 489-505. San Diego: Academic Press.

Yack, J. E., Smith, M. L. and Weatherhead, P. J. (2001) Caterpillar talk: acoustically mediated territoriality in larval Lepidoptera. Proc Natl Acad Sci USA 98: 1137111375

Yager, D. D. (1999) Structure, development, and evolution of insect auditory systems. Micro Res Techniq 47:380-400

Yager, D. D. and Spangler, H. G. (1997) Behavioral response to ultrasound by the tiger beetle Cicindela marutha Dow combines aerodynamic changes and sound production. J Exp Biol 200: 649-659 


\section{APPENDIXI}

Species names and voucher numbers for Costa Rican specimens. Additional information can be found at $<$ http://janzen.sas.upenn.edu/caterpillars/database.lasso $>$

- Acanthodica sinuilinea, 09-SRNP-56485

- Adhemarius ypsilon, 09-SRNP-2628k

- Antirrhea lindigii, 09-SRNP-2705

- Astraptes creteus crana, 09-SRNP-56365

- Automeris phrynon, 09-SRNP-41249

- Automeris postalbida, 09-SRNP-55683

- Danaus sp., 09-SRNP-56453

- Eacles ormondei, 09-SRNP-56205

- Euglyphis sp., 09-SRNP-56167

- Eumorpha satellitia, 09-SRNP-56221

- Lirmiris fascis, 09-SRNP-56384

- Lirimiris guatemalensis, 09-SRNP-56460

- Manduca albiplaga, 09-SRNP-2704

- Manduca floristan, 09-SRNP-56410

- Manduca pellenia, 09-SRNP-2707

- Morpho amathonte, 09-SRNP-56497

- Nyceryx magna, 09-SRNP-2633

- Pachygonidia drucei, 09-SRNP-2635

- Schausiella santarosensis, 09-SRNP-13852; 09-SRNP-13853; 09-SRNP-13854

- Smyrna blomfildia, 09-SRNP-56500

- Unknown noctuid, 09-SRNP-56487

- Xylophanes anubus, 09-SRNP-2634

- Xylophanes tyndarus, 09-SRNP-56477; 09-SRNP-56478 